\title{
Superconducting Proximity Effect in Magnetic Molecules
}

\author{
By \\ Lina Jaurigue
}

\begin{abstract}
A thesis
submitted to the Victoria University of Wellington in fulfilment of the requirements for the degree of

Master of Science
\end{abstract}

Victoria University of Wellington

2013 


\begin{abstract}
We studied the transport through magnetic molecules (MM) coupled to superconducting $(\mathrm{S})$, ferromagnetic $(\mathrm{F})$ and normal $(\mathrm{N})$ leads, with the aim of investigating the interplay between the magnetism and the superconducting proximity effect. The magnetic molecules were modeled using the Anderson model with an exchange coupling between the electron spins and the spin of the molecule. We worked in the infinite superconducting gap limit and treated the coupling between the molecule and the superconducting lead exactly, via an effective Hamiltonian. For the F/N-MM-S systems we used a real-time diagrammatic perturbation theory to calculate the electronic transport properties of the systems to first order in the tunnel coupling to the normal or ferromagnetic lead and then analysed the properties with respect to the parameters of these models. For these systems we found that the current maps out the excitation energies of the eigenstates of the effective Hamiltonian and that various parameters in these systems can lead to a negative differential conductance. In the N-MM-S case the current had no overall spin dependence, but when the normal lead is instead ferromagnetic there was a spin dependence and both the electronic and molecular spin expectation values could take on non-zero values. We also found that the polarisation of the ferromagnetic lead suppresses the superconducting proximity effect. Furthermore in the N-MM-S case the Fano factor indicated a transition from Poissonian transport of single electrons to Poissonian transport of electron pairs as the superconducting proximity effect goes out of resonance, however in the F-MM-S case this did not occur. For the S-MM-S systems we calculated the equilibrium Josephson current and found that in the infinite superconducting gap limit no $0-\pi$ transition was possible. Advantages of this study compared to related ones are that we allow for arbitrarily large Coulomb interactions and we take into account coupling to the superconducting lead non-perturbatively. This is however at the expense of working in the superconducting gap limit. Recently it has been possible to couple single molecules to superconducting leads. This study therefore aims to be indicative of the transport properties that will be observed in future experiments involving single magnetic molecules coupled to leads.
\end{abstract}




\section{Acknowledgements}

I thank my supervisor Michele for all he has taught me and for all the questions he has answered.

I thank Victoria University and the MacDiarmid Institute for financial support.

I thank Stephan Meyer and Walter Somerville for helping me with Latex.

I thank Stephanie Droste for discussions.

I thank Jonnel for his support. 


\section{Contents}

1 Introduction $\quad 2$

1.1 Magnetic Molecules . . . . . . . . . . . . . . . . . . . . . . . 4

1.1.1 Anderson Model . . . . . . . . . . . . . . . . . . 5 5

1.1.2 Quantum Dots . . . . . . . . . . . . . . 6

1.2 Superconductivity, AB States and the Josephson Effect . . . . . . . . . 7

1.2.1 BCS Theory . . . . . . . . . . . . . . . . . 8

1.2.2 Andreev Reflection . . . . . . . . . . . . . . . . . . 10

1.2.3 Josephson Current . . . . . . . . . . . . . . . . . . 11

1.3 Electronic Transport Through Molecules and QDs . . . . . . . . . . . . 12

1.4 Outline . . . . . . . . . . . . . . . . . . . . . 17

2 Real-time Keldsyh Diagram Expansion $\quad 19$

2.1 Master Equation . . . . . . . . . . . . . . . . . . . . . . . . 20

2.2 Current . . . . . . . . . . . . . . . . . 25

2.3 Full Counting Statistics . . . . . . . . . . . . . . . . . . 26

3 IMM Coupled to Normal and BCS Leads 29

3.1 N-IMM-S System . . . . . . . . . . . . . . . . . . . . . . . . . . 30

3.1.1 Effective Hamiltonian . . . . . . . . . . . . . . . . . 33

3.2 Transition Rates and Current . . . . . . . . . . . . . . . . . 37

3.3 Results . . . . . . . . . . . . . . . . . . . . . . . 40

3.3.1 Equilibrium and Zero Exchange Coupling Limits . . . . . . . . . 40

3.3.2 Andreev Current . . . . . . . . . . . . . . . . . 41

3.3 .3 Fano Factor . . . . . . . . . . . . . . . . . . . . . 46

3.4 N-IMM-S Conclusions . . . . . . . . . . . . . . . . . . . . 47

4 AMM Coupled to Normal and BCS Leads 49

4.1 N-AMM-S System . . . . . . . . . . . . . . . . . . . . . . 49 
4.2 Transition Rates and Current . . . . . . . . . . . . . . . 52

4.3 Results . . . . . . . . . . . . . . . . . . . . . 53

4.4 N-AMM-S Conclusions . . . . . . . . . . . . . . . . 55

5 Josephson Current $\quad 59$

5.1 S-MM-S Systems . . . . . . . . . . . . . . . . . . . 59

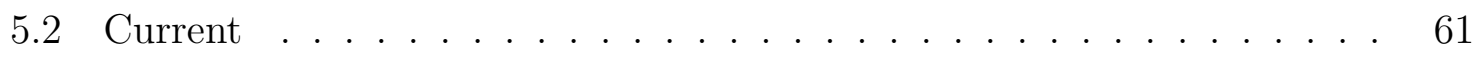

5.3 Results . . . . . . . . . . . . . . . . . . . 62

5.4 Josephson Current Conclusions . . . . . . . . . . . . . . . . . 65

6 IMM Coupled to Ferromagnetic and BCS Leads $\quad 67$

6.1 F-IMM-S System . . . . . . . . . . . . . . . . . 68

6.2 Transition Rates and Current . . . . . . . . . . . . . . . . . . 71

6.2.1 Collinear . . . . . . . . . . . . . . . 72

6.2 .2 Non-collinear . . . . . . . . . . . . . . . . 73

6.3 Results - Collinear . . . . . . . . . . . . . . . . 77

6.3.1 Ferromagnetic Lead, $\mathrm{B}=0$. . . . . . . . . . . . 77

6.3.2 External Magnetic Field, $\mathrm{P}=0$. . . . . . . . . . . . 85

6.3.3 Ferromagnetic Lead and External Magnetic Field . . . . . . . . 89

6.4 Results - Non-collinear . . . . . . . . . . . . . . . . . . . . . . 90

6.4.1 Dependence of Current on $\Gamma_{F} \ldots \ldots \ldots 1$

6.4.2 Effect of Polarisation and Alignment of the Magnetisation of the Ferromagnetic Lead $-B=\Gamma_{S}=2 J \ldots \ldots . \ldots . \ldots 93$

6.4.3 Varying $J, B$ and $\Gamma_{S}$, and the Effect of Shifting the Off Diagonal Reduced Density Matrix Element Resonances . . . . . . . . . . 102

6.5 F-IMM-S Conclusions . . . . . . . . . . . . . . . . . 105

$\begin{array}{lll}7 & \text { Summary and Conclusions } & 107\end{array}$

\section{Appendix}

$\begin{array}{ll}\text { A Diagrammatic Rules } & 109\end{array}$

$\begin{array}{ll}\text { B Eigenstates of the AMM-S System } & 111\end{array}$

C Generalised Transition Rates - F-IMM-S 115

C.1 Solving Integrals of Generalised Transition Rates . . . . . . . . . . . . 115

C.2 Generalised Transition Rates . . . . . . . . . . . . . . . . . . . 118 


\section{Chapter 1}

\section{Introduction}

As semi-conductor based electronics are reaching their limits there are exciting new possibilities on the horizon. In 1994 Peter Shor showed that a quantum algorithm could exponentially speed up classical computations [1]. Since then researchers around the globe have been working towards the realisation of quantum computing. The fields of spintronics, nano-electronics and molecular electronics play an essential role in achieving this goal, as it is through the manipulation of individual spins, electrons and atoms that devices capable of quantum computing will be made.

Single molecule magnets (SMM) have received a lot attention in the past few years as they are a good platform for developing devices which exhibit spin dependent transport and could therefore be used for quantum information storage and processing [2]. A significant amount of research has been carried out on the transport properties through systems containing quantum dots [3-7]. In comparison the research done on electronic and spin transport through magnetic molecules is still in its early stages. Furthermore, it is only through recent advances in nano-fabrication techniques that experimentalists are now capable of contacting individual molecules to electrodes [8-13].

As with quantum dots, due to the size and relatively few degrees of freedom of an individual molecule, quantisation and charging effects play an important role in transport. To realise transport through molecules they are coupled to leads. Depending on the type of leads different effects can be observed. Experimental work on $\mathrm{C}_{60}$ molecules between superconducting leads has exhibited Josephson currents [14], Kondo correlations [15,16] and Coulomb interaction effects [13]. Quantum dots coupled to superconducting leads have been extensively studied $[5,13,17,18]$ and it has been shown that a superconducting proximity effect induces Andreev bound states [19] in the quantum dot. As magnetism and superconductivity are competing effects it is interesting, 
from not only a practical point of view but also due to the interesting physics that could arise, to investigate the effects of coupling a magnetic molecule to a superconducting lead. For practical applications it is interesting to investigate if such a system can exhibit spin dependent transport, for then the extra degrees of freedom introduced by the spin dependence can be utilised along with the coherent, dissipation-less transport properties of the superconductor. In this thesis we will therefore perform a theoretical study on the electronic transport properties of systems involving a magnetic molecule coupled to a superconducting lead. For simplicity we will focus on an idealised model for the magnetic molecule. Nevertheless, when transport is dominated by the molecular orbital closest to the Fermi level of the leads this model should be predictive of electronic transport through magnetic molecules.

Recent theoretical studies have looked at the Josephson current through isotropic and anisotropic magnetic molecules [20,21]. In these works the models for the molecules contained a single orbital level, an energy cost $U$ for double occupation, which is due to Coulomb interactions, and an exchange coupling between the electronic and molecular spins. References [20] and [21] treated the superconducting gap $\Delta$ as finite, however for simplicity they let $U \rightarrow \infty$. We will be concerned with only sub-gap transport and will therefore let $\Delta \rightarrow \infty$. This will allow us to perform a non-perturbative expansion in the tunnel coupling to the superconducting lead which will let us easily include arbitrarily strong Coulomb interactions. Because we are interested in the effect of superconducting proximity effect on the magnetic molecule we will consider strong coupling to the superconducting lead. In the past, experiments have been conducted on quantum dots coupled to superconducting leads but due to technical difficulties, such as the oxidation between the superconductor and semiconductor interfaces completely suppressing Cooper pair tunneling, these setups were only in the weak coupling regime. However the development of new materials such as carbon nanotubes and self-assembled quantum dots, as well as the ability to couple single molecules to leads, has now made it possible to carried out experiments in the intermediate to strong coupling regimes [3].

In this thesis we investigate the transport properties of the MM-S subsystem coupled to normal, ferromagnetic and superconducting leads. For the case of coupling to a ferromagnetic lead we will look at the effects of applying an external magnetic field and will allow for arbitrary alignment of the magnetisation of the ferromagnetic lead and the external magnetic field. To investigate the transport properties we will calculate the sequential current through these systems by using a real-time diagrammatic perturbation theory. 


\section{$1.1 \quad$ Magnetic Molecules}

In recent years the electronic transport properties of single molecules have attracted a lot of attention, both experimentally and theoretically $[10-13,20-22]$. This is due to advances in nano-fabrication techniques [12] as well as quantum effects, such as tunneling, the Coulomb blockade and the Kondo effect [23], which can be observed in these single molecules. A subset of single molecules that are of particular interest, due to their potential application in spintronics, are molecular magnets.
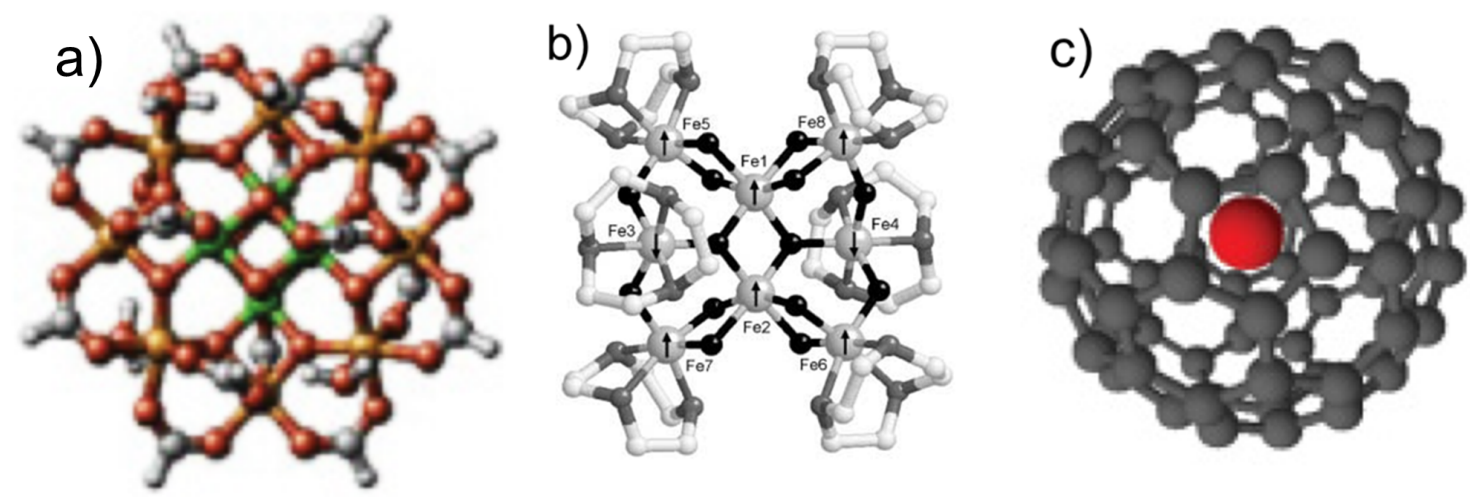

Figure 1.1: Examples of SMMs. a) shows $\mathrm{Mn}_{12}$ [21], b) shows $\mathrm{Fe}_{8}$ [10] and c) shows $\mathrm{N} @ \mathrm{C}_{60}[11]$.

A conventional magnet is usually made of some ferromagnetic metal in which the spins of the electrons are aligned and a macroscopic number of coupled centers are involved. A single molecule magnet (SMM) is very different from a conventional magnet due to the small number of coupled centers and the structure of the molecule; they are strictly speaking not magnets as they are not in the thermodynamic limit. An SMM is defined as a molecule whose magnetization is persistent over long time scales. An example is $\mathrm{Mn}_{12}$ acetate which has a relaxation of magnetisation of the order of months at a temperature of $2 \mathrm{~K}[24]$.

The prototypical SMM is $\mathrm{Mn}_{12}$ acetate $\left(\mathrm{Mn}_{12}\right)$. This molecule consists of organic ligands bonded to 12 manganese ions. There are various derivatives of Mn12 which feature different ligands, one example is $\left[\mathrm{Mn}_{12} \mathrm{O}_{12}\left(\mathrm{O}_{2} \mathrm{C}-\mathrm{C}_{6} \mathrm{H}_{4}-\mathrm{SAc}\right)_{16}\left(\mathrm{H}_{2} \mathrm{O}\right)\right]$ [12]. Figure 1.1 a) shows a schematic diagram of an $\mathrm{Mn}_{12}$ molecule. The total spin of $\mathrm{Mn}_{12}$ is $S=10$ and the molecule has an anisotropy barrier of about $6 \mathrm{meV}$ [25]. Due to the large spin and high anisotropy barrier molecules such as $\mathrm{Mn}_{12}$ show magnetic hysteresis. The high anisotropy barrier is important in achieving long relaxation times of the 
magnetisation of the molecule. Also shown in Fig. 1.1 are diagrams of the structures of $\mathrm{Fe}_{8}$ and $\mathrm{N} @ \mathrm{C}_{60} . \mathrm{Fe}_{8}$ is another $\mathrm{SMM}$ that has received a lot of attention [10]. The formula for this molecule is $\left[\mathrm{Fe}_{8} \mathrm{O}_{2}(\mathrm{OH})_{12}(\operatorname{tacn})_{6}\right] \mathrm{Br}_{8}$ (tacn = 1,4,7-triazacyclononane), it also has total spin $S=10$. For $\mathrm{Fe}_{8}$ the relaxation times becomes long enough to perform direct measurements at temperatures under $1 \mathrm{~K}[26]$. The third molecule, $\mathrm{N} @ \mathrm{C}_{60}$, is a nitrogen atom caged in a $\mathrm{C}_{60}$ molecule. The total spin $S=3 / 2$ of this molecule is much lower than the previously mentioned molecules [11]. Within the $\mathrm{C}_{60}$ cage much of the atomic character of the nitrogen atom is retained, which is often not the case since previous studies involving SMMs have shown that strong interaction with the environment can destroy the molecular magnetism [27]. Due to this there is interest in using the nuclear spin of the nitrogen atom or the electron spin residing on the atom for quantum information processing [2].

SMMs typically have quite a complicated structure (Fig. 1.1). To theoretically model such molecules is very difficult, therefore for general studies on the transport properties of magnetic molecules simplifying approximations are usually made. To reduce the degrees of freedom of the molecule, often only one orbital level is considered [20-23]. This approximation is realistic in a scenario where the spacing between orbital levels is large enough such that only one level is accessible in the energy regime of interest. In this case the model for describing the molecule coupled to leads is based on the Anderson model. The Anderson model was first developed by P. W. Anderson to model localised magnetic states in metals [28] and has since been widely used to model quantum dots and magnetic molecules. The Anderson model is described in the next subsection.

\subsubsection{Anderson Model}

Here we give the Anderson model describing a single orbital level coupled to two metallic systems. The Hamiltonian reads

$$
H_{D}=\sum_{\sigma} \epsilon d_{\sigma}^{+} d_{\sigma}+U n_{\uparrow} n_{\downarrow}
$$

where $d_{\sigma}^{(+)}$is an electron annihilation (creation) operator, $n_{\sigma}=d_{\sigma}^{+} d_{\sigma}$ is the number operator and $\sigma=\uparrow, \downarrow$ is the spin of the electron. The first term describes the occupation of the level, it can either be empty, singly occupied, or doubly occupied. The second term is the Coulomb interaction term, it describes the energy cost of double occupation, $U$. 
To describe the tunneling coupling to the leads the Hamiltonian

$$
H_{t u n n, \eta}=\sum_{k, \sigma}\left(V_{\eta k \sigma} C_{\eta k \sigma}^{+} d_{\sigma}+H . c .\right)
$$

is used. Here $C_{\eta k \sigma}^{+}$is the electron creation operator for lead $\eta=L, R$ and $V_{\eta k \sigma}$ is the tunneling matrix element, which is related to the strength of the coupling. The leads are treated as equilibrium reservoirs and in the case of normal metallic leads they can be modeled by

$$
H_{\eta}=\sum_{k, \sigma} \epsilon_{k} C_{\eta k \sigma}^{+} C_{\eta k \sigma}
$$

This simple model is usually employed to describe transport through quantum dots but can easily be modified to include effects that occur in magnetic molecules. Such effects include exchange coupling between electronic and molecular spins, anisotropy, and quantum tunneling of magnetisation. The Hamiltonians describing the leads can also be modified to describe ferromagnetic or superconducting leads.

\subsubsection{Quantum Dots}

As the model for describing a single orbital magnetic molecule reduces to that of a quantum dot in certain limits, it can be useful to compare the transport properties of these systems. We therefore give a brief introduction to quantum dots in this section.

Quantum dots are made by confining the charge carriers to a small region in a semi-conducting material in all three spatial directions. They contain $10^{3}-10^{9}$ atoms and range in size from several nanometers to microns [29]. Due to their size, their properties are intermediate between bulk semiconductors and atoms and have been referred to as artificial atoms [30]. Striking properties of quantum dots are that both the charge on and the energy of the dot are quantised. Like atoms, quantum dots have well defined energy levels, yet unlike in atoms the level spacing can be tuned. It is this control that makes quantum dots so attractive for a wide range of applications in fields including optics [31], nano-electronics [4] and quantum computing [32-34].

Properties of quantum dots can be investigated by performing electronic transport measurements. To do this the quantum dot is contacted to source and drain electrodes. Figure 1.2 shows a schematic diagram of such a setup. As well as being contacted to the electrodes the quantum dot is also capacitively coupled to a gate. Varying the gate voltage will shift the orbital levels of the quantum dot, thereby controlling which levels are in the energy regime needed for transport. Due to the size of the quantum 
dot Coulomb repulsion effects are strong. For an electron to tunnel onto the dot it must have enough energy to overcome the repulsion due to the electrons already occupying the dot. This is energy, referred as the charging energy, is dependent on the gate voltage. Therefore tuning the gate voltage can control when sequential tunneling can occur and when the system is in the so called Coulomb blockaded regime where transport is forbidden.

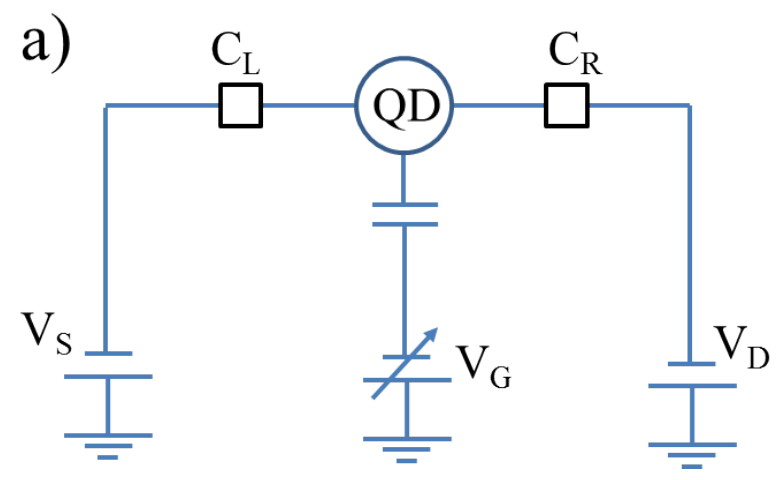

b)

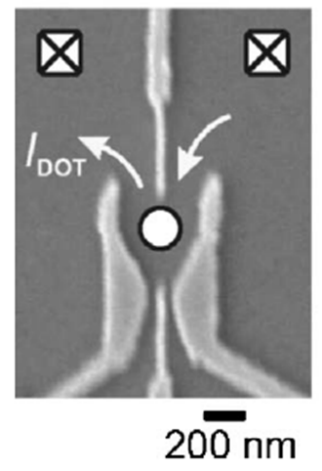

Figure 1.2: a) Schematic diagram of a quantum dot. The boxes represent the tunnel barriers. b) Scanning electron microscope image of a lateral quantum dot [35].

\subsection{Superconductivity, Andreev Bound States and the Josephson Effect}

Superconductivity is a very well-researched phenomenon that was discovered by Heike Kamerlingh Onnes in 1911 [36]. A material in the superconducting state has zero electrical resistance and zero magnetic field in its interior. The exclusion of magnetic field was discovered by Meissner and Ochsenfeld in 1933 and is known as the Meissner effect [37]. Since its discovery much work has been carried out to theoretically describe superconductivity. The first successful microscopic theory of superconductivity was developed by Bardeen, Cooper and Schrieffer [38], the so call BCS theory. In BCS theory it was shown that electrons could form pairs in the presence of just a weak attractive interaction to form a new ground state. The effective interaction is often provided by an electron-phonon interaction. The bound electrons are called Cooper pairs; they have equal and opposite momenta, so that the ground state has zero momentum. To calculate the ground state wavefunction BCS used a mean field approach to describe the dependence of the occupation of one state on all other states. Through this they 
discovered that the formation of bound pairs leads to the gap in the energy spectrum of the superconductor, $2|\Delta|$. The superconducting gap $\Delta$ is temperature dependent. At high temperatures there is no gap and the material is not superconducting, but as the temperature is lowered past the critical temperature the gap is opened. In BCS theory the critical temperature can be found by letting $|\Delta|$ tend to zero. At energies greater than $|\Delta|$, quasiparticles states exist, but these are forbidden for energies less than $|\Delta|$. Within the superconducting gap only Cooper pairs can occupy states. Therefore for energy regimes that are small compared to the superconducting gap only Cooper pairs contribute to transport. In order to single out these subgap contributions the $\Delta \rightarrow \infty$ limit can be taken.

When a molecule or a quantum dot is contacted to a superconducting lead with $\Delta \rightarrow \infty$ only Cooper pairs can tunnel to and from this lead. The process by which this can occur is called Andreev reflection. If the both leads are superconducting then a current can flow through the system even if there in no applied bias. This current, called the Josephson current, flows if there is a phase difference between the two superconductors.

\subsubsection{BCS Theory}

In this subsection we give a brief introduction to BCS theory. More detailed descriptions can be found in texts such as Ref. [39].

In 1956 Cooper showed that at least one bound pair of electrons would form if there is a weak interaction in a Fermi sea of electrons, regardless of how weak that interaction is, as long as it is positive [40]. Therefore, in the presence of a net positive interaction pairs of electrons, known as Cooper pairs, should form until an equilibrium point is reached. To describe the ground state of such a system BCS used the variational wave function

$$
\left|\psi_{G}\right\rangle=\prod_{\mathbf{k}=\mathbf{k}_{1}, \ldots, \mathbf{k}_{\mathbf{M}}}\left(u_{\mathbf{k}}+v_{\mathbf{k}} C_{\mathbf{k} \uparrow}^{+} C_{-\mathbf{k} \downarrow}^{+}\right)\left|\phi_{0}\right\rangle
$$

where $\left|\phi_{0}\right\rangle$ is the vacuum state, $\left|u_{\mathbf{k}}\right|^{2}+\left|v_{\mathbf{k}}\right|^{2}=1$ and $\mathbf{k}=\mathbf{k}_{\mathbf{1}}, \ldots, \mathbf{k}_{\mathbf{M}}$ are the occupied states in momentum space. The coefficients $u_{\mathbf{k}}$ and $v_{\mathbf{k}}$ are related to the probability of pairs being occupied. Using this ground state and the so-called pairing Hamiltonian,

$$
H=\sum_{\mathbf{k} \sigma} \epsilon_{\mathbf{k}} n_{\mathbf{k} \sigma}+\sum_{\mathbf{k l}} V_{\mathbf{k l}} C_{\mathbf{k} \uparrow}^{+} C_{-\mathbf{k} \downarrow}^{+} C_{-\mathbf{l} \downarrow} C_{\mathbf{l} \uparrow}
$$


the coefficients $u_{\mathbf{k}}$ and $v_{\mathbf{k}}$ can be calculated. These are found to be

$$
\left|v_{\mathbf{k}}\right|^{2}=\frac{1}{2}\left(1-\frac{\xi_{\mathrm{k}}}{E_{\mathrm{k}}}\right)
$$

and

$$
\left|u_{\mathbf{k}}\right|^{2}=\frac{1}{2}\left(1+\frac{\xi_{\mathbf{k}}}{E_{\mathbf{k}}}\right)
$$

where $\xi_{\mathbf{k}}=\epsilon_{\mathbf{k}}-\mu_{S}$ is the is single particle energy $\epsilon_{\mathbf{k}}$ relative to the Fermi level $\mu_{S}$, $E_{\mathbf{k}}=\left(\Delta^{2}+\xi_{\mathbf{k}}^{2}\right)^{1 / 2}$ and $\Delta$ is related to the pairing potential $V_{\mathbf{k} \mathbf{l}}$ which is chosen to be $-V$ for states below a cut-off energy $\hbar \omega_{c}$ and zero otherwise. In Ginzburg-Landau theory for superconductivity $\Delta$ is the order parameter and contains a phase factor $e^{i \varphi}$, where $\varphi$ is the phase difference between $u_{\mathbf{k}}$ and $v_{\mathbf{k}}$. This leads to the BCS mean field Hamiltonian

$$
H_{S}=\sum_{\mathbf{k} \sigma}\left(\epsilon_{\mathbf{k}}-\mu_{S}\right) C_{\mathbf{k} \sigma}^{+} C_{\mathbf{k} \sigma}-\Delta \sum_{\mathbf{k}}\left(C_{-\mathbf{k} \downarrow} C_{\mathbf{k} \uparrow}+C_{\mathbf{k} \uparrow}^{+} C_{-\mathbf{k} \downarrow}^{+}\right) .
$$

for an s-wave superconductor. This Hamiltonian does not conserve particle number, but for the purposes of this work this does not matter since we treat the superconducting lead as an equilibrium reservoir with a fix electrochemical potential. $H_{S}$ is quadratic in electron operators and can therefore be diagonalised. To do this the Bogoliubov quasi-particle operators

$$
\begin{gathered}
\gamma_{\mathbf{k} \uparrow} \equiv u_{\mathbf{k}} c_{\mathbf{k} \uparrow}-v_{\mathbf{k}} c_{-\mathbf{k} \downarrow}^{+}, \\
\gamma_{\mathbf{k} \downarrow} \equiv u_{\mathbf{k}} c_{\mathbf{k} \downarrow}+v_{\mathbf{k}} c_{-\mathbf{k} \uparrow}^{+} .
\end{gathered}
$$

are introduced. The creation operators are the Hermitian conjugates. Using these fermionic quasi-particle operators, with the definitions of $u_{\mathbf{k}}$ and $v_{\mathbf{k}}$ given above, neglecting an irrelevant constant the BCS Hamiltonian can be cast into the form

$$
H_{S}=\sum_{\mathbf{k} \sigma} E_{\mathbf{k}} \gamma_{\mathbf{k} \sigma}^{+} \gamma_{\mathbf{k} \sigma}
$$

where $E_{\mathbf{k}}$ has the same definition as above and has turned out to be the quasi-particle excitation energy. From the equation for $E_{\mathbf{k}}$ we can see that $\Delta$ is half the width of the gap in the single particle density of states of the superconductor, as $|\Delta|$ is the minimum energy a quasi-particle can have. Figure 1.3 shows the quasi-particle density of states of a superconductor. For energies less than $|\Delta|$ either side of the Fermi level there are no single particle states; in this energy range only Cooper pairs are allowed. 


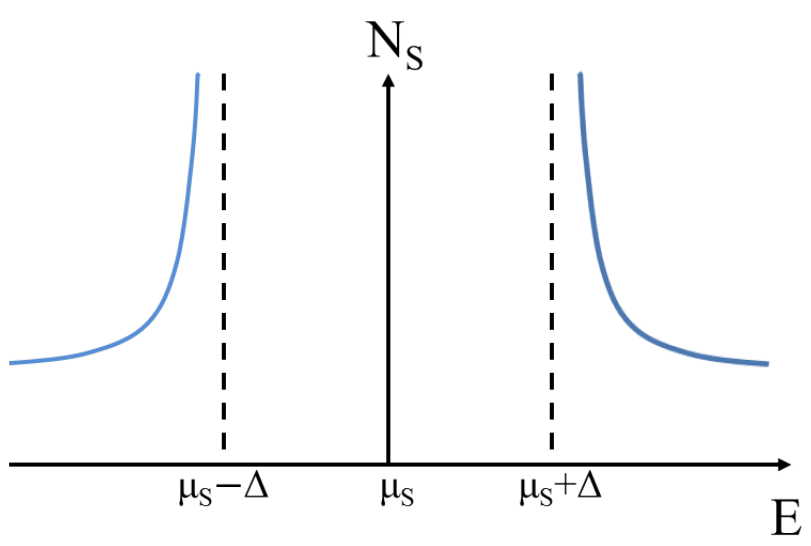

Figure 1.3: The single particles density of states for a superconductor, $N_{S}$, shows a symmetric gap about $\mu_{S}$ with a width of $2|\Delta|$. Out side the gap quasi-particle excitations are possible, however at energies inside the gap only Cooper pairs are allowed.

\subsubsection{Andreev Reflection}

When a normal metal is contacted to a superconductor then superconductivity can be induced in the normal metal. This is known as the proximity effect [41] and has been known of since the 1930s [42]. If the Fermi level of the metal lies within the gap of the superconductor then a single electron cannot enter the superconductor from the metal as there are no available states. Therefore if an electron is incident on the boundary between the metal and the superconductor it must be reflected (Fig. 1.4). This electron can be reflected in the form of a hole with the opposite velocity of the incident electron and the opposite spin. This means that both momentum and spin are conserved in this reflection process. In this process a Cooper pair is transferred into the superconductor, which makes up for the charge $2 e$ that is lost in the reflection process. This process is called Andreev reflection $[43,44]$ and is a convenient way to explain the process by which a Cooper pair can pass between the two materials. When the normal metal is in between two superconducting leads then the hole that is reflected from one boundary must be reflected as an electron from the opposite boundary. This process happens repeatedly and can lead to constructive interference of the incident and reflected electron waves, forming a so called Andreev bound state [19].

If there is a molecule or a quantum dot contacted to a superconducting lead then the same process occurs. However in this case it is the orbital levels of the molecule or quantum dot that are relevant, rather than the Fermi level of the metal. 


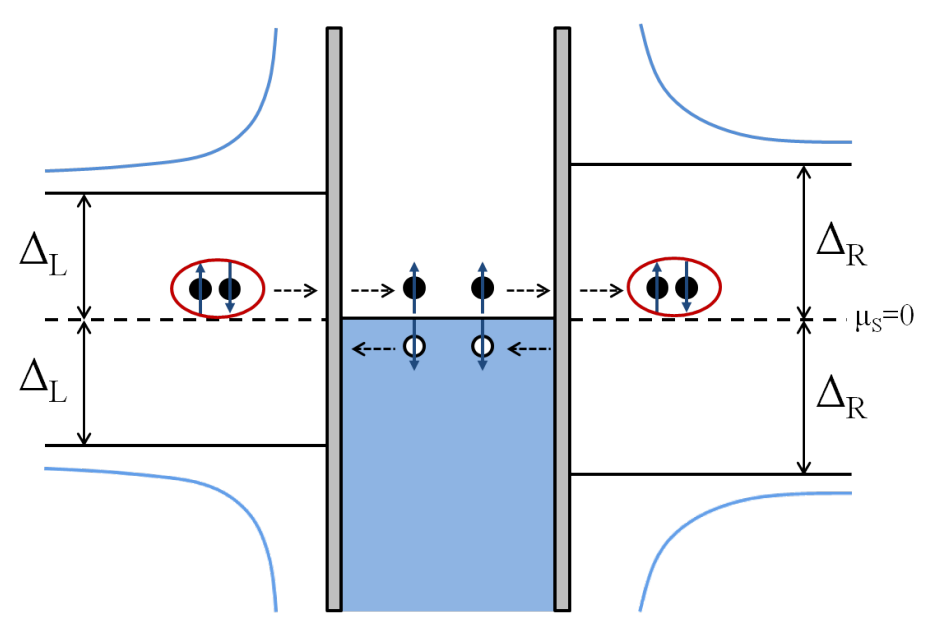

Figure 1.4: This diagram shows an S-N-S junction. The Fermi level of the normal metal lies in the gap of the two superconducting regions, meaning that a single electron cannot enter the superconductors. The electron impinging on the boundary to the right superconductor is reflected as a hole and a charge of $2 e$ is transfered to the superconductor. The reflected hole is then reflected as an electron at the left boundary and in this process a charge of $2 e$ leaves the left superconductor.

\subsubsection{Josephson Current}

In 1962 Brian Josephson predicted what is now known as the Josephson effect [14]. The Josephson effect allows a supercurrent to flow between two superconducting regions separated by an insulating region or a non-superconducting metallic region when there is no applied voltage, as long as there is a difference between the phases of the order parameters of the two superconducting regions. Josephson perdicted that the current

$$
I_{j o s}=I_{c} \sin \varphi
$$

would flow when no bias voltage is applied. Here $\varphi$ is the phase difference between the two superconducting regions and $I_{c}$ is the critical current, i.e. the maximum supercurrent that can flow across the junction. Josephson also predicted that the phase difference would vary with an applied voltage according to

$$
\frac{d \varphi}{d t}=\frac{2 e V}{\hbar}
$$

causing an AC Josephson current. We will concentrate on the DC Josephson current, details on the AC Josephson current can be found in Ref. [39]. 
The electrical work done by the current source is

$$
W=F=\int I_{j o s} V d t=\int I_{j o s} \frac{\hbar}{2 e} d \varphi
$$

where $F$ is the free energy stored in the junction. Rearranging this equation we find

$$
I_{j o s}=-\frac{2 e}{\hbar} \frac{\partial F}{\partial \phi}
$$

If the states of the system in question are discrete then the free energy can be calculated using

$$
F=-k_{B} T \ln Z
$$

where

$$
Z=\sum_{i} e^{-\frac{E_{i}}{k_{B} T}}
$$

is the partition function and $E_{i}$ is the energy of state $i$. Substituting the free energy and the partition function into Eq. 1.15, the Josephson current can be expressed as

$$
I_{j o s}=\frac{2 e}{\hbar} k_{B} T \frac{\partial}{\partial \phi} \ln Z=-\frac{2 e}{\hbar} \sum_{i} \frac{\partial E_{i}}{\partial \phi} \frac{e^{-\frac{E_{i}}{k_{B} T}}}{Z} .
$$

The critical current in Eq. 1.12 can be positive or negative. When $I_{c}>0$ the Josephson phase $\varphi$ of the junction is zero in the ground state (when no current is flowing). When $I_{c}<0$ Eq. 1.12 can be rewritten as

$$
I_{j o s}=-\left|I_{c}\right| \sin \varphi=\left|I_{c}\right| \sin (\varphi+\pi) .
$$

Here we see that $\varphi=\pi$ when $I_{j o s}=0$. When this is the case the junction is said to be a $\pi$-Josephson junction. Under certain circumstances a Josephson junction can transition between the zero and $\pi$ phases.

\subsection{Electronic Transport Through Molecules and Quantum Dots}

In this section we will address how electronic transport through molecules and quantum dots is realised. We will then review some of the relevant experimental and theoretical work that has be done in the fields of nano and molecular electronics. 


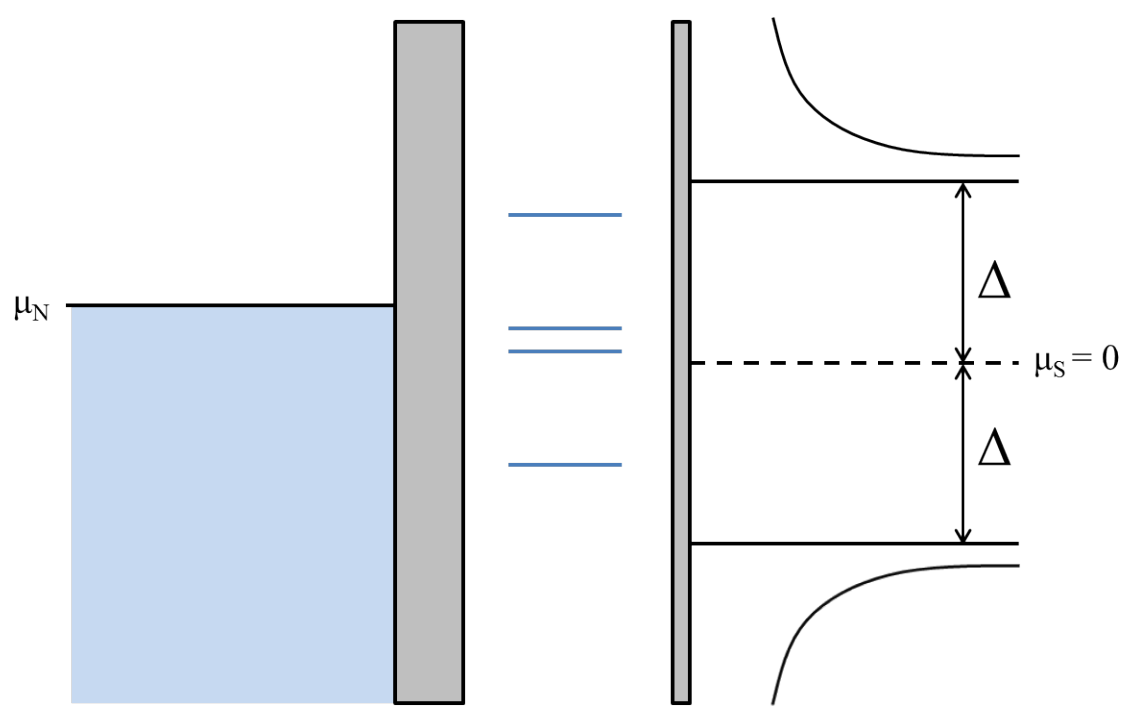

Figure 1.5: A molecule coupled to a normal metal lead and a superconducting lead. The blue lines in the central region indicate the orbital levels of the molecule. The states of the molecule that contribute to the current are those that lie within the bias window set by the two leads.

To study the electronic transport properties through a molecule (or a quantum dot) it must be contacted to at least two conducting leads. These leads can be superconducting, magnetic or normal metallic. In each case, for current to flow the energy levels of the molecule must lie within the bias window set by the leads. Figure 1.5 shows a schematic diagram of a molecule weakly coupled to a normal metallic lead on the left and strongly coupled to a superconducting lead on the right. The levels of the molecule lie within the gap of the superconductor, meaning that no single particles can tunnel between the superconducting lead and the molecule. In an experimental setup the energy levels of the molecule could be tuned by applying a gate voltage. The choice of material for the leads depends on the effects to be investigated. With superconducting leads interesting effects include the proximity, Josephson, and Kondo effects. Ferromagnetic leads can cause spin-dependent transport, spin accumulation on the molecule and a ferromagnetic proximity effect. Some of these effects can be used to probe properties of the molecule through which transport is occurring [45] [46] [20]. Systems which contain a combination of lead types are also of interest for pure physics reasons, and for the possibility of developing devices with new properties which could be useful in areas such as spintronics, quantum computing and optics.

In this thesis we investigate the transport properties of a magnetic molecule coupled to a superconducting lead and a second lead which can either be a normal metal, 
ferromagnetic or superconducting. For the remainder of this section we will review some of the theoretical and experimental research that has been carried out on systems containing combinations of the aforementioned components.

Most experimental work that has been carried out on SMMs has involved normal metallic leads, gold is commonly used. Heersche et al. have performed transport measurements through a single $\mathrm{Mn}_{12}$ molecule coupled to gold electrodes. They observed negative differential conductance features on the energy scale of the anisotropy barrier, something they had not previously observed with other molecules or bare gold samples. Figure 1.6 shows an SEM image of the type of devices they have tested. The molecule is too small to be resolved but it sits in the gap between the two gold electrodes. Heersche et al. found that with a simple model that incorporates the anisotropy of the molecule and the quantum tunneling of magnetisation they were able to qualitatively understand current and differential conductance features in the sequential tunneling regime [12]. The current calculations we will perform in this work will also be in the sequential tunneling limit for coupling to a normal lead. Roch et al. have carried out experimental and theoretical studies on $\mathrm{N} @ \mathrm{C}_{60}$ coupled to gold electrodes. They calculated the current in both the sequential tunneling regime and the cotunneling regime. In both cases they found good qualitative agreement between their experimental and their theoretical results and that an anti-ferromagnetic exchange between the nitrogen atom and the $\mathrm{C}_{60}$ molecule best fits the data [11].

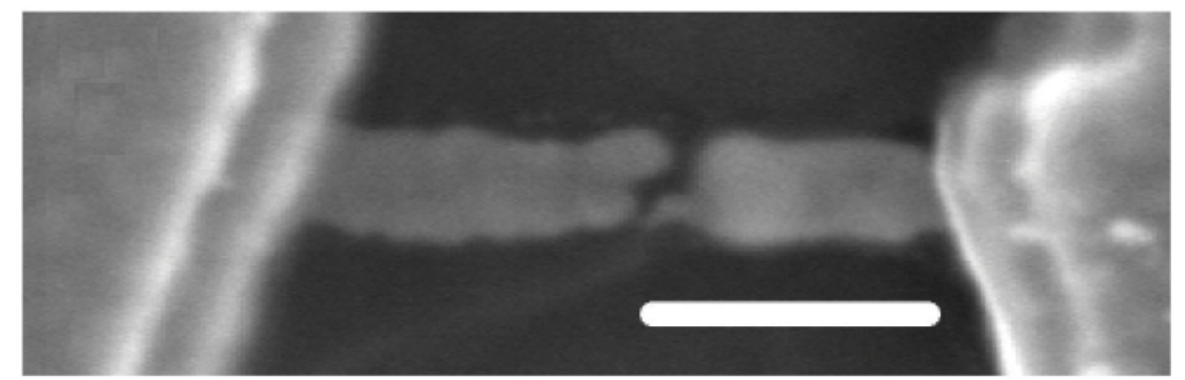

Figure 1.6: A scanning electron microscope (SEM) image of a $\mathrm{Mn}_{12}$ molecule contacted to two gold electrodes. The scale bar corresponds to $200 \mathrm{~nm}$ and the width of the molecule is about $3 \mathrm{~nm}[12]$.

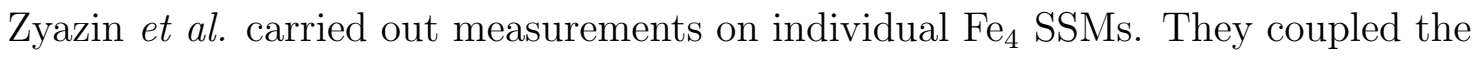
molecule to three gold electrodes to perform three-terminal transport measurements in the presence of an external magnetic field. The ground states spin of $\mathrm{Fe}_{4}$ is $S=5$ and this is retained when the molecule is deposited on gold. Using the transport 
measurements they were able to make estimates of the easy axis anisotropy constant and the anisotropy barrier of the molecule and demonstrated that, via an electric field, they could control the anisotropy of an SMM [9]. The ability to control properties of SMMs is important for their use in applications such as quantum computing. Parks et al. have also reported the experimental confirmation of mechanical control of the spin states and anisotropy of a SMM; in this case a Cobalt complex [8].

The experimental work discussed so far has involved only normal metallic leads. We will now discuss two experimental studies that involve superconducting and ferromagnetic leads, neither of these studies however involve SMMs. Winkelmann et al. have carried out electronic transport measurements on $\mathrm{C}_{60}$ molecules coupled to superconducting leads. The superconducting leads are made of either aluminium or gold in the proximity of an aluminium capping layer. They engineered samples with weak to strong coupling to the leads and demonstrated the coexistence and competition of superconductivity and Kondo correlations for varying coupling strength and external magnetic field magnitudes [13]. This work paves the way for similar experiments involving endofullerenes such as $\mathrm{N} @ \mathrm{C}_{60}$. The second study was carried out by Hofstetter et al.. They studied the ferromagnetic proximity effect in a quantum dot coupled to a superconducting and a ferromagnetic lead. Figure 1.7 shows an image of a typical devices they have constructed. Through transports measurements they were able to demonstrate that a local exchange field is induced in the quantum dot due to the ferromagnetic lead. They used the Kondo effect to probe the local exchange field. With respect to the energy regime they were working in the superconducting lead had a finite gap [45].

A lot of theoretical research has has been carried out on electronic transport through quantum dots. Sothmann et al. studied the transport properties of a quantum dot coupled to two ferromagnetic leads and one superconducting lead. They worked in the infinite superconducting gap limit, considered a finite Coulomb interaction and allowed for arbitrary alignment of the ferromagnetic leads. The F-QD-F subsystem forms a quantum dot spin valve, a device that has received a lot of experimental and theoretical interest due to the spin dependence of electronic transport through this system. They were able show that by introducing the superconducting lead the exchange field induced by the proximity to the ferromagnetic lead could be experimentally probed [46]. Sothmann et al. have used the same real-time diagrammatic technique that we use in this thesis. The same diagrammatic approach is used in [5] and [47] to calculate the current through quantum dots coupled to normal and superconducting 


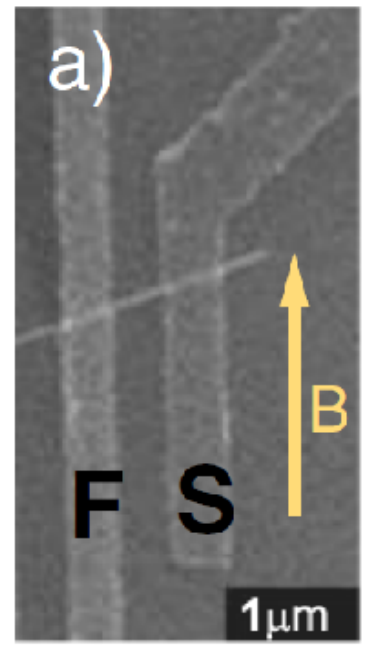

Figure 1.7: A SEM image of an InAs nanowire contacted to a Ti/Al bilayer superconducting lead and a $\mathrm{Ni} / \mathrm{Co} / \mathrm{Pd}$ trilayer ferromagnetic lead, with an external magnetic field applied parallel to the easy axis of the ferromagnetic lead [45].

leads. Governale et al. model an interacting quantum dot coupled to a normal and two superconducting leads. In the infinite superconducting gap limit they found that there is a $\pi$ transition in the non-equilibrium Josephson current, which can be triggered by both the voltage of the normal lead and the gate voltage, which controls the level position [5]. Braggio et al. performed a theoretical study on an interacting quantum dot coupled to a normal metallic lead and a superconducting lead. They also worked in the limits of infinite superconducting gap and finite Coulomb interaction. To study the superconducting proximity effect in this system they used full counting statistics to obtain the current and the zero frequency noise. They found that the Fano factor changes from 2 to 1 as the superconducting proximity effect goes from off-resonance to resonance conditions. This suggests Poissonian transport in both regimes, one electron transport on resonance as the transport is limited by single electron tunneling events between the dot and the normal lead, and two electron transport off resonance as in this regime the current is limited by the tunneling of electron pairs to and from the superconducting lead [47]. In the limit of zero exchange coupling between the electronic and molecular spins the dynamics of the single level isotropic magnetic molecule (IMM) in an N-IMM-S system reduce to those of the systems studied in [5] and [47].

In the theoretical work of Lee et al. the Josephson effect through an isotropic magnetic molecule is investigated. They work with a finite superconducting gap and 
model the magnetic molecule as a single level quantum dot with an exchange interaction between molecular spin and the electron spin. They use a numerical renormalisation approach to calculate non-perturbative low temperature transport properties. To do this they work in the regime of infinite Coulomb interaction between electrons on the molecule, meaning that the level of the molecule can only be singly occupied or empty. They find that when the superconducting gap exceeds the Kondo temperature the Josephson junction is in the $\pi$ state, however with sufficiently large antiferromagnetic exchange coupling the 0 state is restored. Due to the asymmetry in the behaviour with the exchange coupling Lee et al. suggest that the sign of the coupling could be determined experimentally [20]. In this thesis we use the same model for the isotropic magnetic molecule as is used by Lee et al. However we work in a different regime. As we will work in the $\Delta \rightarrow \infty$ limit transport will only be possible through Andreev reflection processes. On the other hand, in the work of Lee et al. transport must occur in the cotunneling regime. The results of the for the two regimes will therefore be quite different. Sadovskyy et al. have studied a similar system to that in [20], however their system is generalised to an anisotropic magnetic molecule. The model they used consisted of a single level magnetic molecule in the presence of an external magnetic field, coupled to two finite gap superconducting leads. They also worked in the infinite Coulomb interaction limit. They used a perturbation expansion in the tunnel coupling to the leads to calculate the Josephson current and also found that with anti-ferromagnetic coupling between the electronic and molecular spins a $\pi-0$ transition can be induced. They also find that it is possible to obtain information of the anisotropy of the molecule by studying the critical current [21]. Other studies involving SMMs demonstrate that a transport spectroscopy of a SMM coupled to normal and/or ferromagnetic leads shows signs of quantum tunneling [48] and negative differential conductance features [22].

\subsection{Outline}

The aim of thesis is to investigate the electronic transport properties through systems comprised of a magnetic molecule coupled to a superconducting lead and a second lead that can be normal, ferromagnetic or superconducting, with the purpose of adding new knowledge to the fields of nano and molecular electronics. To calculate the nonequilibrium sequential current through these systems we will use a real-time diagrammatic approach and work in the $\Delta \rightarrow \infty$ limit. We will work in the regime of strong 
coupling to the superconducting leads and weak coupling to the normal or ferromagnetic leads. The coupling to the superconducting leads will be treated exactly by using an effective Hamiltonian. Our main focus will be on the Andreev current but we will also calculate the zero frequency noise and briefly look at the Josephson current in the case where the molecules are coupled to two superconducting leads.

In Chapter 2 we introduce the diagrammatic perturbation theory that will be used in the subsequent chapters. In this chapter we also introduce full counting statistics and explain how the zero frequency noise and the Fano factor can be calculated. Next, in Chapter 3 we investigate an isotropic magnetic molecule coupled to a normal and a superconducting lead. The spin of the molecule is incorporated into the model via an exchange interaction between the electronic and molecular spins. We first introduce the model then give the derivation for the effective Hamiltonian describing the coupling to the superconducting lead. Using the eigenstates of the effective Hamiltonian we then calculate the sequential current caused by the couping to the normal lead. In Chapter 4 we modify the system slightly to allow for the molecule to be anisotropic. Then in Chapter 5 we use the models for the isotropic and anisotropic molecules of the pervious two chapters and investigate the Josephson current through these molecules. Following this, in Chapter 6 we are once again concerned with the electronic transport through an isotropic magnetic molecule, however this time coupled to a superconducting lead and a ferromagnetic lead in the presence of an external magnetic field. We investigate the cases of magnetisation of the ferromagnetic lead and the external magnetic field being collinear and non-collinear. Finally, in Chapter 7 we summaries the results of the previous four chapters. 


\section{Chapter 2}

\section{Real-time Keldsyh Diagram Expansion}

In the following section a diagrammatic perturbation theory for a molecule, or a quantum dot, with strong interactions, contacted to non-interacting leads in non-equilibrium conditions and at finite temperature, is given. The Hamiltonian of such a system is of the form

$$
H=H_{L}+H_{M}+H_{T} \equiv H_{0}+H_{T},
$$

where $H_{L}$ describes the leads, $H_{M}$ describes the molecule and $H_{T}$ are the tunneling terms. The perturbation expansion that will be performed is with respect to the tunneling Hamiltonian. The general idea of the theory is to split the density matrix of the system into two parts, one describing the leads, which have many degrees of freedom but are non-interacting, and the other describing the molecule, which is interacting but has only a few degrees of freedom. Because the leads are non-interacting they can be integrated out using Wick's theorem, leaving the much smaller system of the molecule to be treated exactly. The time evolution of the remaining reduced density matrix is described by a master equation in Liouville space, the elements of which can be calculated using diagrammatic techniques.

The advantage of this technique is that allows one to calculate non-equilibrium dynamics, include arbitrarily strong Coulomb interactions, and easily treat off-diagonal density matrix elements. In some cases this technique also allows non-perturbative expansions in the tunnel coupling. This is the case with BCS leads in the $\Delta \rightarrow \infty$ limit, the expansion can be exactly summed to all orders in tunneling coupling.

The diagrammatic perturbation expansion can be used to calculate the elements of the reduced density matrix, as well as the current. With a minor adjustment to the 
theory full counting statistics can also be calculated. In this chapter we will first derive the master equation of the reduced density matrix elements [49] then show how the current can be calculated. Lastly, we will show how the full counting statistic can be calculated, with an emphasis on the Fano factor.

\subsection{Master Equation}

The expectation value of an observable at time $t$ is given by

$$
\langle A(t)\rangle=\operatorname{Tr}\left[A(t)_{H} \rho_{0}\right]
$$

where $\rho_{0}$ is the initial density matrix of the system and $A_{H}(t)$ is the observable at time $t$ in the Heisenberg representation. We assume that at the initial time $t_{0}$ the denstiy matrix can be factorised into parts, for the molecule (or dot) $\rho_{0}^{M}$ and the leads $\rho_{0}^{r}$;

$$
\rho_{0}=\rho_{0}^{M} \prod_{r=L, R} \rho_{0}^{r}
$$

The leads are treated as equilibrium reservoirs with fixed Fermi levels $\mu_{r}$ and can therefore be described using the Fermi function $f(\omega)$ and the equilibrium density matrix

$$
\rho_{0}^{r}=\frac{1}{Z_{0}^{r}} e^{-\beta\left(H_{r}-\mu_{r} N_{r}\right)}
$$

Here $\beta=1 / k_{B} T$, where $k_{B}$ is the Boltzmann factor, $N_{r}$ is the number operator and $H_{r}=\sum_{k, \sigma} \epsilon_{k} C_{r k \sigma}^{+} C_{r k \sigma}$ is the Hamiltonian that describes the leads with annihilation (creation) operators $C_{r \sigma}^{(+)}$and energies $\epsilon_{k}$. In the case of a superconducting lead the Hamiltonian is that given in Eq.1.11. The normalisation factor $Z_{0}^{r}$ is determined by the condition $\operatorname{Tr}\left[\rho_{0}^{r}\right]=1$. The initial density matrix describing the molecule can be chosen to be diagonal in an appropriate basis $\{|\chi\rangle\}$ and is given by

$$
\rho_{0}^{M}=\sum_{\chi} P_{\chi}^{(0)}|\chi\rangle\langle\chi|
$$

where $P_{\chi}^{(0)}$ are the initial occupation probabilities of the states $|\chi\rangle$ and $\sum_{\chi} P_{\chi}^{(0)}=1$. We are interested in the stationary limit, $t_{0} \rightarrow-\infty$. In this limit, at time $t$, all observables are independent of the choice of the initial probabilities $P_{\chi}^{(0)}$. Furthermore, choosing the initial density matrix to be diagonal does not mean it must remain so at some later 
time $t$.

Next it is useful to go from the Heisenberg picture to the interaction picture. The aim of this section is to perform a perturbation expansion in the tunnel coupling between the molecule and the leads, therefore we treat the tunneling Hamiltonians as the perturbation when we change to the interaction picture. The tunneling Hamiltonian is of the form of Eq. 1.2. Doing this gives us

$$
A(t)_{H}=\tilde{T} e^{i \int_{t_{0}}^{t} d t^{\prime} H_{T}\left(t^{\prime}\right)_{I}} A(t)_{I} T e^{-i \int_{t_{0}}^{t} d t^{\prime} H_{T}\left(t^{\prime}\right)_{I}},
$$

where $T(\tilde{T})$ is the (anti-)time ordering operator and we have set $\hbar=1$. Substituting this into Eq. 2.2 we get

$$
\langle A(t)\rangle=\operatorname{Tr}\left[\tilde{T} e^{i \int_{t_{0}}^{t} d t^{\prime} H_{T}\left(t^{\prime}\right)_{I}} A(t)_{I} T e^{-i \int_{t_{0}}^{t} d t^{\prime} H_{T}\left(t^{\prime}\right)_{I}} \rho_{0}\right] .
$$

Reading from the right we start with $\rho_{0}$ then propagate forward in time up to $t$, the time at which the expectation value of $A(t)$ is calculated, then we propagate backwards in time back to $t_{0}$. This is represented diagrammatically in Fig. 2.1. This time curve is referred to as the Keldysh contour and propagation along this contour can be written more compactly by introducing the Keldysh time ordering operator $T_{K}$, which acts on all operators to the right of it,

$$
\begin{aligned}
& \langle A(t)\rangle=\operatorname{Tr}\left[T_{K} \exp \left(-i \int_{K} d t^{\prime} H_{T}\left(t^{\prime}\right)\right) A(t)_{I} \rho_{0}\right] . \\
& \overbrace{\text { time } \longrightarrow} \overbrace{T \operatorname{Texp}\left(i \int_{t_{0}}^{t} d t H_{T}\left(t^{\prime}\right)_{I}\right)}^{T \exp \left(-i \int_{t_{0}}^{t} d t H_{T}\left(t^{\prime}\right)_{I}\right)}\} A(t)_{I}
\end{aligned}
$$

Figure 2.1: A diagrammatic representation of Eq. 2.7. Along the top path the system is propagated forward to the time when the observable is measured, then backward along the bottom path to the initial time.

To calculate the elements of the reduced density matrix of the molecule $P_{\chi_{2}}^{\chi_{1}}(t)$ we must find the expectation value of the projection operator $\left|\chi_{2}\right\rangle\left\langle\chi_{1}\right|(t)$. Replacing $A(t)$ 
in Eq. 2.8 with $\left|\chi_{2}\right\rangle\left\langle\chi_{1}\right|(t)$ we find

$$
P_{\chi_{2}}^{\chi_{1}}(t)=\left\langle\mid \chi_{2}\right\rangle\left\langle\chi_{1} \mid(t)\right\rangle=\operatorname{Tr}\left[T_{K} \exp \left(-\int_{K} d t^{\prime} H_{T}\left(t^{\prime}\right)_{I}\right)\left|\chi_{2}\right\rangle\left\langle\chi_{1}\right|(t)_{I} \rho_{0}\right] .
$$

Writing out the trace over the states of the molecule in terms of the sum over the states and the using Eqs. 2.3 and 2.5, this can be written as

$$
P_{\chi_{2}}^{\chi_{1}}(t)=\sum_{\chi_{1}^{\prime}, \chi_{2}^{\prime}}\left\langle\chi_{2}^{\prime}\left|\operatorname{Tr}_{\text {leads }}\left[T_{K} \exp \left(-i \int_{K} d t^{\prime} H_{T}\left(t^{\prime}\right)_{I}\right)\left|\chi_{2}\right\rangle\left\langle\chi_{1}\right|(t)_{I} \prod_{r=L, R} \rho_{0}^{r}\right]\right| \chi_{1}^{\prime}\right\rangle P_{\chi_{2}^{\prime}}^{\chi_{1}^{\prime}}\left(t_{0}\right)
$$

It is now useful to define the full propagator of the the system as

$$
\Pi_{\chi_{2} \chi_{2}^{\prime}}^{\chi_{1} \chi_{1}^{\prime}}\left(t, t_{0}\right)=\left\langle\chi_{2}^{\prime}\left|\operatorname{Tr}_{\text {leads }}\left[T_{K} \exp \left(-i \int_{K} d t^{\prime} H_{T}\left(t^{\prime}\right)_{I}\right)\left|\chi_{2}\right\rangle\left\langle\chi_{1}\right|(t)_{I} \prod_{r=L, R} \rho_{0}^{r}\right]\right| \chi_{1}^{\prime}\right\rangle
$$

Equation 3.6 can now be compactly written as

$$
P_{\chi_{2}}^{\chi_{1}}(t)=\sum_{\chi_{1}^{\prime}, \chi_{2}^{\prime}} \Pi_{\chi_{2} \chi_{2}^{\prime}}^{\chi_{1} \gamma_{1}^{\prime}}\left(t, t_{0}\right) P_{\chi_{2}^{\prime}}^{\chi_{1}^{\prime}}\left(t_{0}\right)
$$

The next step is to expand the time-ordered expotential,

$$
T_{K} \exp \left(-i \int_{K} d t^{\prime} H_{T}\left(t^{\prime}\right)_{I}\right)=\sum_{n=0}^{\infty} \frac{(-i)^{n}}{n !} \int_{K} d t_{1} \ldots \int_{K} d t_{n} T_{K}\left[H_{T}\left(t_{1}\right)_{I} \ldots H_{T}\left(t_{n}\right)_{I}\right]
$$

The lead Hamiltonians are bilinear in the creation and annihilation operators of the lead electrons (or quasiparticles in the case of a superconducting lead). This means Wick's theorem can be applied. Performing pairwise contractions of the lead operators in the tunneling Hamiltonians can be represented diagrammatically by placing internal vertices (black dots) on the Keldysh contour at every position where a tunneling Hamiltonian arises from the expansion of the time-ordered exponential and a directed tunnel line (a black line with an arrow head) indicating the contraction of two lead operators. The tunnel lines point to the vertex where an electron is created on the molecule. The observable is indicated on the contour by an external vertex (open circle) at time $t$. The Hamiltonian of the molecule is not bilinear in the electron operators, meaning that Wick's theorem does not apply and the operations of the tunneling Hamiltonian on the states of the molecule must be worked out explicitly. This can be 
done by keeping track of the state of the molecule along the contour. Figure 2.2 shows this diagrammatic representation of the time evolution of the reduced system.

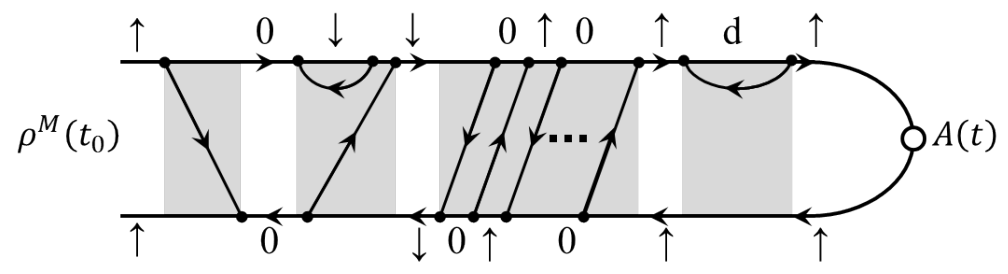

Figure 2.2: The time evolution of the reduced density matrix is shown in this example. Along the top path the reduced system propagates forward in time from $t_{0}$ to $t$, at which time the observable $A(t)$ is measured, then the system propagates along the bottom contour back to time $t_{0}$. Along the contour, vertices indicate the tunneling Hamiltonian terms that have arisen from the expansion of the exponential, Eq. 2.13. Each is connected to one other tunneling Hamiltonian term and the change in the state of the molecule due to the tunneling event is indicated by the state of the molecule before and after each tunneling event.

The diagram in Fig. 2.2 can be broken up into two types of blocks, irreducible selfenergies $W_{\chi_{2} \chi_{2}^{\prime}}^{\chi_{1} \chi_{1}^{\prime}}\left(t, t^{\prime}\right)$ and free propagators $\Pi_{\chi_{2} \chi_{2}^{\prime}}^{(0) \chi_{1} \chi_{1}^{\prime}}\left(t, t^{\prime}\right)$. Irreducible self-energies are parts of the diagram where any vertical cut would intersect a tunneling line and the free propagators are the parts where any vertical cut intersects no tunneling lines. The irreducible self-energies represent the transition from $P_{\chi_{2}^{\prime}}^{\chi_{1}^{\prime}}\left(t^{\prime}\right)$ to $P_{\chi_{2}}^{\chi_{1}}(t)$ which occur due to tunneling events to and from the leads. The free propagators represent free time evolution of the reduced system and are given by

$$
\Pi_{\chi_{2} \chi_{2}^{\prime}}^{(0)} \chi_{1} \chi_{1}^{\prime}\left(t, t^{\prime}\right)=\delta_{\chi_{1} \chi_{1}^{\prime}} \delta_{\chi_{2} \chi_{2}^{\prime}} e^{-i\left(\epsilon_{1}-\epsilon_{2}\right)\left(t-t^{\prime}\right)}
$$

where $\epsilon_{1}\left(\epsilon_{2}\right)$ is the energy of the eigenstate $\left|\chi_{1}\right\rangle\left(\left|\chi_{2}\right\rangle\right)$.

The full propagator is obtained by summing over all combinations of the free propagators and the irreducible self-energies, represented diagrammatically in Fig. 2.3. The resulting Dyson equation for the full propagator is given by

$\Pi_{\chi_{2} \chi_{2}^{\prime}}^{\chi_{1} \chi_{1}^{\prime}}\left(t, t^{\prime}\right)=\Pi_{\chi_{2}}^{(0) \chi_{1}}\left(t, t^{\prime}\right) \delta_{\chi_{1} \chi_{1}^{\prime}} \delta_{\chi_{2} \chi_{2}^{\prime}}+\sum_{\chi_{1}^{\prime \prime} \chi_{2}^{\prime \prime}} \int_{t^{\prime}}^{t} d t_{2} \int_{t^{\prime}}^{t_{2}} d t_{1} \Pi_{\chi_{2}}^{(0) \chi_{1}}\left(t, t_{2}\right) W_{\chi_{2} \chi_{2}^{\prime \prime}}^{\chi_{1} \chi_{2}^{\prime \prime}}\left(t_{2}, t_{1}\right) \Pi_{\chi_{2}^{\prime \prime} \chi_{2}^{\prime}}^{\chi_{1}^{\prime \prime} \chi_{1}^{\prime}}\left(t_{1}, t^{\prime}\right)$.

For convenience we introduce the notation $X_{\chi_{2} \chi_{2}}^{\chi_{1} \chi_{1}}=X_{\chi_{2}}^{\chi_{1}}$, where $X$ can be a free propagator or an irreducible self-energy. 


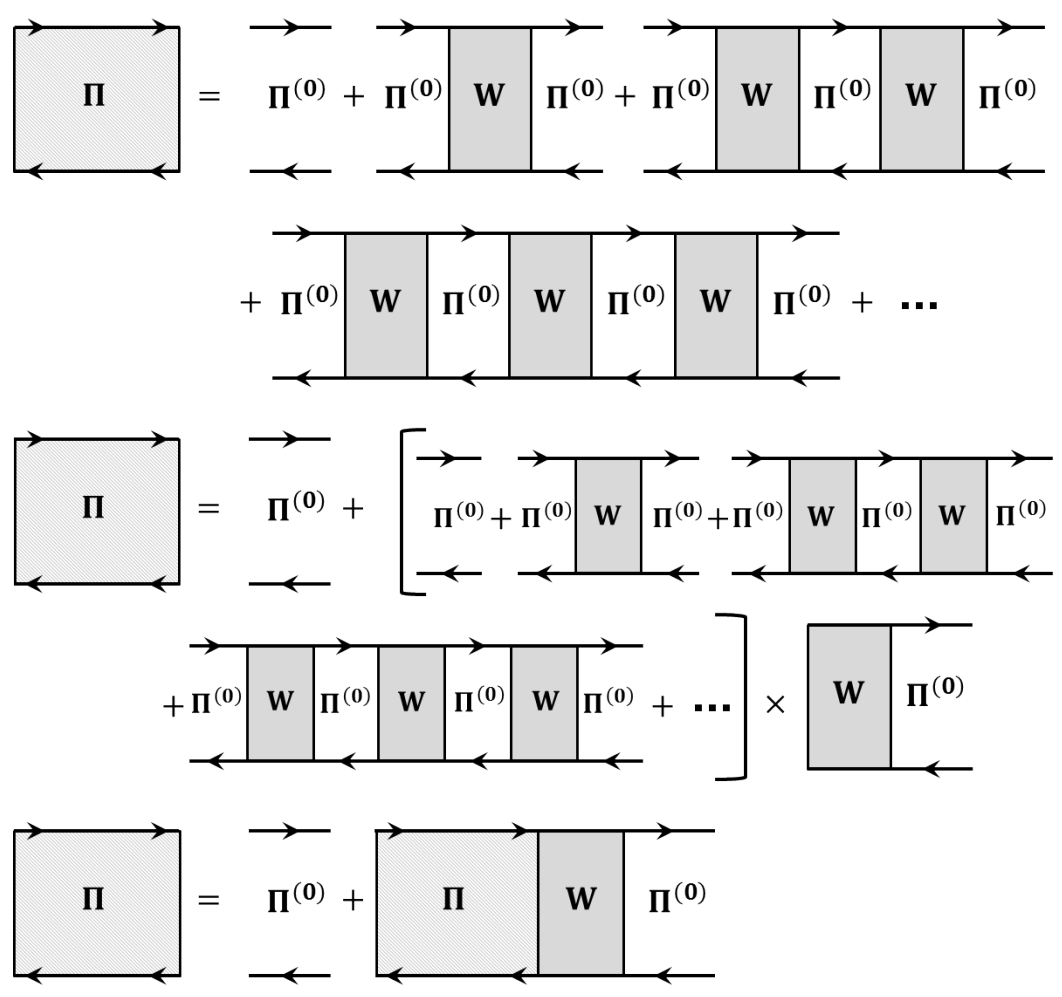

Figure 2.3: Summing over all combinations of free propagators and irreducible selfenergies gives a Dyson equation for the full propagator.

Using Eqs. 2.12 and 2.15 the time evolution of the reduced density matrix can now be written as $P_{\chi_{2}}^{\chi_{1}}(t)=\Pi_{\chi_{2}}^{(0) \chi_{1}}\left(t, t^{\prime}\right) P_{\chi_{2}}^{\chi_{1}}\left(t^{\prime}\right)+\sum_{\chi_{1}^{\prime} \chi_{2}^{\prime} \chi_{1}^{\prime \prime} \chi_{2}^{\prime \prime}} \int_{t^{\prime}}^{t} d t_{2} \int_{t^{\prime}}^{t_{2}} d t_{1} \Pi_{\chi_{2}}^{(0)}{\underset{\chi}{1}}_{2}\left(t, t_{2}\right) W_{\chi_{2} \chi_{2}^{\prime \prime}}^{\chi_{1} \chi_{1}^{\prime \prime}}\left(t_{2}, t_{1}\right) \Pi_{\chi_{2}^{\prime \prime} \chi_{2}^{\prime}}^{\chi_{1}^{\prime \prime} \chi_{1}^{\prime}}\left(t_{1}, t^{\prime}\right) P_{\chi_{2}^{\prime}}^{\chi_{1}^{\prime}}\left(t^{\prime}\right)$.

To get the dynamics, or a generalised master equation, of the reduced density we differentiate this equation with respect to $t$, giving

$$
\dot{P}_{\chi_{2}}^{\chi_{1}}(t)=-i\left(\epsilon_{\chi_{1}}-\epsilon_{\chi_{2}}\right) P_{\chi_{2}}^{\chi_{1}}(t)+\int_{t_{0}}^{t} d t^{\prime} \sum_{\chi_{1}^{\prime} \chi_{2}^{\prime}} W_{\chi_{2} \chi_{2}^{\prime}}^{\chi_{1} \chi_{1}^{\prime}}\left(t, t^{\prime}\right) P_{\chi_{2}^{\prime}}^{\chi_{1}^{\prime}}\left(t^{\prime}\right)
$$

The first term on the right side of the equation describes the coherent evolution of the reduced system, whereas the second term describes dissipative coupling to the leads. In the stationary limit, where the system has no memory of the initial state of the system, we take $t_{0} \rightarrow-\infty$. $P_{\chi_{1}}^{\chi_{1}}$ at time $t$ depends on the state of the system at earlier times $t^{\prime}$, however in the stationary limit the elements of the reduced density matrix are 
not changing, meaning $P_{\chi_{1}}^{\chi_{1}}(t)=P_{\chi_{1}}^{\chi_{1}}\left(t^{\prime}\right)$. In this limit we can rewrite Eq. 2.17 as

$$
\dot{P}_{\chi_{2}}^{\chi_{1}}(t)=-i\left(\epsilon_{\chi_{1}}-\epsilon_{\chi_{2}}\right) P_{\chi_{2}}^{\chi_{1}}(t)+P_{\chi_{2}^{\prime}}^{\chi_{1}^{\prime}}(t) \int_{-\infty}^{t} d t^{\prime} \sum_{\chi_{1}^{\prime} \chi_{2}^{\prime}} W_{\chi_{2} \chi_{2}^{\prime}}^{\chi_{1} \chi_{1}^{\prime}}\left(t, t^{\prime}\right) .
$$

If there is no explicit time dependence in the system then the self-energies only depend on the time difference $t-t^{\prime}$. Defining $\tau=t-t^{\prime}$ the integral of the kernel becomes $\int_{0}^{\infty} d \tau W_{\chi_{2} \chi_{2}^{\prime}}^{\chi_{1} \chi_{1}^{\prime}}(\tau)$. Introducing the factor $\mathrm{e}^{-z \tau}$ into the integral, with $z=0^{+}$, gives the Laplace transform of the kernel

$$
W_{\chi_{2} \chi_{2}^{\prime}}^{\chi_{1} \chi_{1}^{\prime}}=\left.\int_{0}^{\infty} d \tau \mathrm{e}^{-z \tau} W_{\chi_{2} \chi_{2}^{\prime}}^{\chi_{1} \chi_{1}^{\prime}}(\tau)\right|_{z=0^{+}}
$$

which we define as the so called generalised transition rates. In the stationary limit Eq. 2.17 then becomes

$$
0=-i\left(\epsilon_{\chi_{1}}-\epsilon_{\chi_{2}}\right) P_{\chi_{2}}^{\chi_{1}}+\sum_{\chi_{1}^{\prime} \chi_{2}^{\prime}} W_{\chi_{2} \chi_{2}^{\prime}}^{\chi_{1} \chi_{1}^{\prime}} P_{\chi_{2}^{\prime}}^{\chi_{1}^{\prime}}
$$

The generalised transition rates $W_{\chi_{2} \chi_{2}^{\prime}}^{\chi_{1} \chi_{1}^{\prime}}$ can be calculated using a set of diagrammatic rules or using Fermi's golden rule when the rates are to first order in the tunnel coupling and $\chi_{1}=\chi_{2}\left(\chi_{1}^{\prime}=\chi_{2}^{\prime}\right)$. Diagrammatic rules are given in Appendix A and in Chapter 6 .

\subsection{Current}

The current through each of the leads is given by

$$
\hat{I}_{r}=-e \frac{d \hat{N}_{r}}{d t}=-i e\left[H, \hat{N}_{r}\right]=-i e \sum_{k \sigma} V_{r} C_{r k \sigma}^{+} d_{\sigma}+H . c .
$$

Apart from a factor and a sign difference in front of one of the terms, Eq. 2.21 is the same as the tunneling Hamiltonians. The current can therefore be calculated in a very similar way to the generalised transition rates.

The derivation of the equation for the current is very similar to that for the projection operator. Instead of inserting the projection operator into Eq. 2.8 the current operator is inserted. This gives

$$
I_{r}(t)=\left\langle\hat{I}_{r}(t)\right\rangle=\operatorname{Tr}\left[T_{K} \exp \left(-\int_{K} d t^{\prime} H_{T}\left(t^{\prime}\right)_{I}\right) \hat{I}_{r}(t) \rho_{0}\right]
$$


By inserting the identity this equation can be rewritten as

$I_{r}(t)=\sum_{\chi \chi_{1}^{\prime}, \chi_{2}^{\prime}}\left\langle\chi_{2}^{\prime}\left|\operatorname{Tr}_{\text {leads }}\left[T_{K} \exp \left(-i \int_{K} d t^{\prime} H_{T}\left(t^{\prime}\right)_{I}\right) \hat{I}_{r}(t)|\chi\rangle\langle\chi| \prod_{r=L, R} \rho_{0}^{r}\right]\right| \chi_{1}^{\prime}\right\rangle P_{\chi_{2}^{\prime}}^{\chi_{1}^{\prime}}\left(t_{0}\right)$

As the current operator terms are of the same form as the tunneling Hamiltonian terms, this equation is very similar to Eq.3.6. The subsequent manipulation of this equation is the same as what is carried out to obtain the master equation of the density matrix elements, and leads to the following equation for the current -

$$
I_{r}=-e \sum_{\chi \chi_{1}^{\prime} \chi_{2}^{\prime}} W_{\chi \chi_{2}^{\prime}}^{\chi \chi_{1}^{\prime r}} P_{\chi_{2}^{\prime}}^{\chi_{1}^{\prime}}
$$

The diagrammatic rules for calculating the generalised current rates, $W_{\chi \chi_{2}^{\prime}}^{\chi \chi_{1}^{\prime r}}$, are slightly different to those for calculating the generalised transition rates as they must account for a sign difference when an electron is created or destroyed in the lead. They must also ensure that each current diagram is counted only once. The current vertex appears at the end of the Keldysh contour at time $t$ and is contracted with a tunneling vertex. This diagram can be draw in block form in one of two ways, as shown in Fig. 2.4, and only one of these should be included in the current calculation. These diagrams also show that the generalised current rates must always end in diagonal terms.

\subsection{Full Counting Statistics}

In a 2006 publication Braggio et al. presented a theory of full counting statistics for electronic transport in systems with interacting electrons [6]. This theory presents a way of calculating not only the current in the system but the full transport properties. The information on the transport properties is contained in the probability distribution $P(N, t)$ that $N$ charges have passed through the system in time $t . P(N, t)$ is related to the current, the noise and higher order moments of the distribution cumulants. These properties can all be conveniently derived using the cumulant generating function (CGF) which is defined as

$$
S(\xi)=-\ln \left[\sum_{N=-\infty}^{\infty} e^{i N \xi} P(N, t)\right]
$$




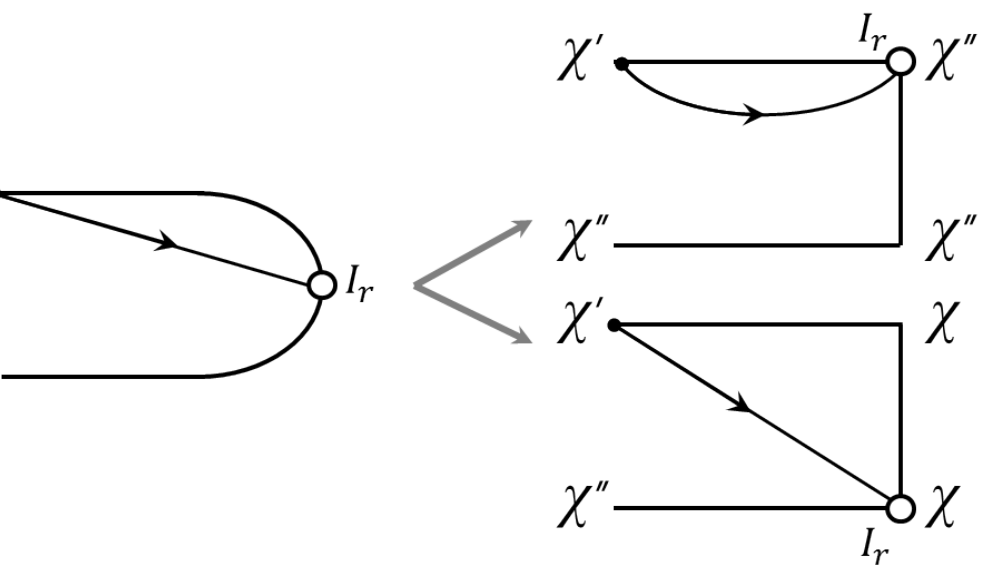

Figure 2.4: These diagrams show the contraction of an external current vertex with an internal tunneling vertex. The current vertex appears at the end of the Keldysh contour at time $t$. This curved contour can be drawn in block form in the two ways that are shown. In both cases the diagram must end in diagonal terms. The equations for the two block diagrams are the same and only one is needed to calculate the current.

where $\xi$ is the counting field. The counting field is used to keep track of the number of charges that have passed through the system and can be introduced into the generalised transition rates by multiplying each term by $e^{ \pm i N \xi}$, where $N$ is the number of charges transferred and the sign depends on whether an electron is leaving or entering the metallic lead. We are only concerned with sequential tunneling, in which case $N= \pm 1$. The derivatives of the CGF,

$$
\langle\langle I\rangle\rangle_{n}=-\left.\frac{(-i e)^{n}}{t} \partial_{\xi}^{n} S(\xi)\right|_{\xi=0}
$$

give the transport information for the system. The first cumulant gives the average current and the second is the zero-frequency noise.

Braggio et al. showed that when there are no off-diagonal reduced density matrix elements, there is an alternative to using Eq. 2.25. They found that to first order in the tunnel coupling the CGF is given by

$$
S^{(1)}(\xi)=-t \lambda^{(1)}(\xi)
$$

where $\lambda^{(1)}(\xi)$ is the eigenvalue of the matrix of first order generalised transition rates $\mathbf{W}$ which has the smallest absolute real part. 
Dividing the second cumulant by $e$ times the first,

$$
F=-\left.i \frac{\partial_{\xi} \lambda^{(1)}(\xi)}{\partial_{\xi}^{2} \lambda^{(1)}(\xi)}\right|_{\xi=0},
$$

gives the Fano factor. If the transport is Poissonian then the Fano factor gives the charge of the carriers. For example if we have Poissonian transfer of electrons in a system without superconductors then the Fano factor will be 1. If we have a system with two superconducting leads and Cooper pairs are being transfered in a Poissonian manner then the Fano factor will be 2 . 


\section{Chapter 3}

\section{Isotropic Magnetic Molecule Coupled to Normal and BCS Leads}

In this chapter we study an isotropic magnetic molecule coupled to one superconducting lead and one normal metallic lead (N-IMM-S). Any realistic magnetic molecule will have more than one orbital level, however due to the complexity of a many level system we will consider a theoretical description with only one orbital level. This being said such a description could be valid for a molecule that has large level spacing compared to the energy regime of transport through that system. In recent work Lee et al. calculated the low temperature transport properties of a single orbital isotropic magnetic molecule coupled to superconducting leads using a numerical renormalisation approach [20]. As this approach is computationally expensive they worked in the limit of infinitely strong Coulomb interactions. We will use the perturbation theory introduced in Chapter 2 and work in the limit of an infinite superconducting gap, which will allow us to derive an effective Hamiltonian for the coupling of the superconducting lead to the magnetic molecule. The advantage here is that we can allow for an arbitrarily strong Coulomb interaction on the molecule. In the first section of this chapter we will introduce the theoretical description of the N-IMM-S system. Then we will derive the effective Hamiltonian. Understanding the states of the effective Hamiltonian will be very important to understand the transport properties of this system, as this Hamiltonian contains information on the transport of Cooper pairs to and from the superconducting lead. We will then calculate the current to first order in coupling to the normal lead, as well as the zero frequency noise, and analyse these results. 


\section{$3.1 \quad$ N-IMM-S System}

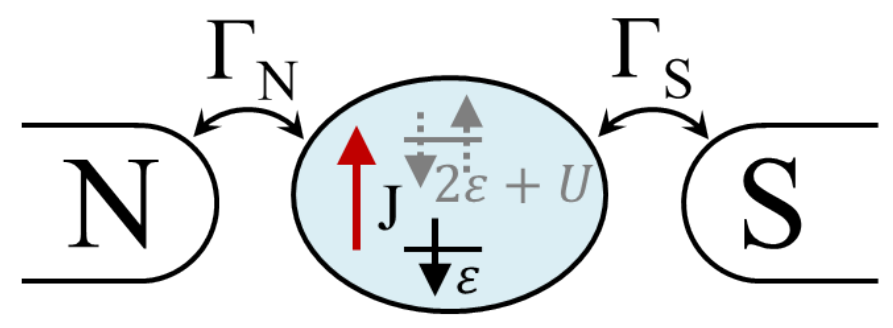

Figure 3.1: A single level magnetic molecule coupled to a superconducting lead and a normal lead, with coupling strengths $\Gamma_{S}$ and $\Gamma_{N}$, and exchange coupling, $J$, between the spin of the electrons occupying the orbital level and the spin of the rest of the molecule.

We consider an isotropic magnetic molecule (IMM) between a superconducting lead and a normal lead, as depicted in Fig. 3.1. The Hamiltonian for this system is

$$
H=H_{M}+H_{N}+H_{S}+H_{t u n n, N}+H_{t u n n, S}
$$

where $H_{M}$ is the Hamiltonian for the molecule, $H_{\eta}$ are the Hamiltonians for the normal, $\eta=N$, and superconducting, $\eta=S$, leads and $H_{t u n n, \eta}$ are the Hamiltonians that describe the tunneling of electrons between the molecule and the leads.

The Hamiltonian describing the molecule is given by

$$
H_{M}=\sum_{\sigma} \epsilon d_{\sigma}^{+} d_{\sigma}+U n_{\uparrow} n_{\downarrow}+J \mathbf{S} . \mathbf{s}_{\mathbf{e}}
$$

This Hamiltonian describes a single orbital molecule with coupling between the spin of the electron in the orbital level, $\mathbf{s}_{\mathbf{e}}$, and the spin of the rest of the molecule, $\mathbf{S}$. It is very similar to the Hamiltonian describing the quantum dot in the Anderson model (Eq. 1.1), the only difference being the addition of the last term, which describes the exchange coupling between the molecular spin and the electronic spin. Once again $d_{\sigma}$, $d_{\sigma}^{+}$are the creation and annihilation operators for electrons with spin $\sigma=\uparrow, \downarrow$. The strength of the exchange coupling is given by $J$. The same model was used in Ref. [20].

The components of $\mathbf{s}_{\mathbf{e}}$ can be written in terms of creation and annihilation operators 
by using the general equation

$$
\left(\mathbf{s}_{\mathbf{e}}\right)_{\mu}=\frac{1}{2}\left(\begin{array}{ll}
d_{\uparrow}^{+} & d_{\downarrow}^{+}
\end{array}\right) \sigma_{\mu}\left(\begin{array}{c}
d_{\uparrow} \\
d_{\downarrow}
\end{array}\right) .
$$

Here $\sigma_{\mu}$ represents the $x, y$ and $z$ Pauli matrices. Using this equation the components of $\mathbf{s}_{\mathbf{e}}$ are

$$
\begin{aligned}
s_{e_{x}} & =\frac{1}{2}\left(d_{\uparrow}^{+} d_{\downarrow}+d_{\downarrow}^{+} d_{\uparrow}\right), \\
s_{e_{y}} & =\frac{i}{2}\left(-d_{\uparrow}^{+} d_{\downarrow}+d_{\downarrow}^{+} d_{\uparrow}\right)
\end{aligned}
$$

and

$$
s_{e_{z}}=\frac{1}{2}\left(d_{\uparrow}^{+} d_{\uparrow}-d_{\downarrow}^{+} d_{\downarrow}\right) .
$$

With these expressions $\mathbf{S} . \mathbf{s}_{\mathbf{e}}$ can be written as

$$
\mathbf{S} . \mathbf{s}_{\mathbf{e}}=\frac{1}{2} S_{-} d_{\uparrow}^{+} d_{\downarrow}+\frac{1}{2} S_{+} d_{\downarrow}^{+} d_{\uparrow}+\frac{1}{2} S_{z}\left(n_{\uparrow}-n_{\downarrow}\right),
$$

where $S_{ \pm}=S_{x} \pm i S_{y}$ are the raising and lowering operators for the spin of the molecule. Note that for simplicity we have set $\hbar=1$ and will do this in all subsequent chapters.

We are considering a molecule with only one orbital level (or sufficient separation from higher levels such that these can be neglected). This level can either be empty, singly occupied or doubly occupied. The states of the isolated molecule are

$$
\begin{gathered}
|0, \alpha\rangle=|0\rangle_{e} \otimes|\alpha\rangle, \\
|\sigma, \alpha\rangle=d_{\sigma}^{+}|0\rangle_{e} \otimes|\alpha\rangle=d_{\sigma}^{+}|0, \alpha\rangle
\end{gathered}
$$

and

$$
|d, \alpha\rangle=d_{\uparrow}^{+} d_{\downarrow}^{+}|0\rangle_{e} \otimes|\alpha\rangle=d_{\uparrow}^{+} d_{\downarrow}^{+}|0, \alpha\rangle,
$$

where $\alpha$ is the spin of the molecule, $|0\rangle_{e}$ is the vacuum state for the molecule and $d$ represents double occupation. To calculate the eigenstates we must now specify what the spin of the molecule is. Most SMMs have large molecular spins [10], for example $\mathrm{Mn}_{12}$ with $S=10$. To model such a large spin would result in a cumbersomely large Hilbert space and as we are taking a first look at the physics of a SMM coupled to normal and superconducting leads, for simplicity we will choose the smallest possible spin to show the effects of an exchange interaction between the electronic and molecular spins. Hence for the remainder of this chapter we will choose $S=\frac{1}{2}$. Using the ba- 
$\operatorname{sis}\{|0,1 / 2\rangle,|d, 1 / 2\rangle,|\uparrow, 1 / 2\rangle,|\downarrow,-1 / 2\rangle,|\downarrow, 1 / 2\rangle,|\uparrow,-1 / 2\rangle,|0,-1 / 2\rangle,|d,-1 / 2\rangle\}$ the matrix form of $H_{M}$ is

$$
H_{M}=\left(\begin{array}{cccccccc}
0 & 0 & 0 & 0 & 0 & 0 & 0 & 0 \\
0 & 2 \epsilon+U & 0 & 0 & 0 & 0 & 0 & 0 \\
0 & 0 & \epsilon+\frac{J}{4} & 0 & 0 & 0 & 0 & 0 \\
0 & 0 & 0 & \epsilon+\frac{J}{4} & 0 & 0 & 0 & 0 \\
0 & 0 & 0 & 0 & \epsilon-\frac{J}{4} & \frac{J}{2} & 0 & 0 \\
0 & 0 & 0 & 0 & \frac{J}{2} & \epsilon-\frac{J}{4} & 0 & 0 \\
0 & 0 & 0 & 0 & 0 & 0 & 0 & 0 \\
0 & 0 & 0 & 0 & 0 & 0 & 0 & 2 \epsilon+U
\end{array}\right)
$$

and the eigenstates are $\left|0, \pm \frac{1}{2}\right\rangle,\left|d, \pm \frac{1}{2}\right\rangle,|T+\rangle=|\uparrow, 1 / 2\rangle,|T-\rangle=|\downarrow,-1 / 2\rangle,|T 0\rangle=$ $\frac{1}{\sqrt{2}}(|\downarrow, 1 / 2\rangle+|\uparrow,-1 / 2\rangle)$ and $|S\rangle=\frac{1}{\sqrt{2}}(|\downarrow, 1 / 2\rangle-|\uparrow,-1 / 2\rangle)$. Due to the exchange coupling between the spin- $\frac{1}{2}$ electrons and molecular spin, the singly occupied states form a triplet and a singlet.

The Hamiltonian for the leads is given by

$$
H_{\eta}=\sum_{k, \sigma} \epsilon_{k} C_{\eta k \sigma}^{+} C_{\eta k \sigma}-\delta_{\eta, S} \Delta \sum_{k}\left(C_{\eta-k \downarrow} C_{\eta k \uparrow}+H . c .\right)
$$

where $\eta$ can either be $\mathrm{N}$ or $\mathrm{S}$ for the normal and superconducting leads. The $C_{\eta k \sigma}^{(+)}$ terms are the creation and annihilation operators in the leads. The second term comes from BCS mean-field theory for superconductors, $2 \Delta$ is the gap of the quasi-particle density of state in the superconductor.

The tunnel coupling to the leads is described by

$$
H_{t u n n, \eta}=\sum_{k, \sigma}\left(V_{\eta} C_{\eta k \sigma}^{+} d_{\sigma}+H . c .\right)
$$

where $V_{\eta}$ are the tunnel matrix elements, which for simplicity are assumed to be independent of the wave number $k$ and spin $\sigma$. The tunnel coupling strengths are defined as $\Gamma_{\eta}=2 \pi N_{\eta}\left|V_{\eta}\right|^{2}$, where $N_{\eta}$ is the density of states of lead $\eta$. As we will calculate the current by treating the tunnel coupling to the normal lead as a perturbation, the coupling strength $\Gamma_{N}$ must be smaller than $k_{B} T$, where $T$ is the temperature and $k_{B}$ the Boltzmann constant. In the subsequent section we will derive the effective Hamiltonian for the molecule coupled to the superconducting lead in the $\Delta \rightarrow \infty$ limit. In this limit the coupling to the superconducting lead can be taken into account non- 
perturbatively, so the strength of $\Gamma_{S}$ can be arbitrary. However, as we are interested in observing the superconducting proximity effect in the molecule, we will work in the regime $\Gamma_{S}>>\Gamma_{N}$. Because we are only dealing with one superconducting lead, without loss of generality, we set the electrochemical potential of the superconducting lead equal zero, $\mu_{S}=0$, and use it as a reference energy.

\subsubsection{Effective Hamiltonian}

We are only interested in the sub-gap transport to and from the superconducting lead. Therefore we will make the simplifying approximation that $\Delta \rightarrow \infty$. Due to this approximation the affect the superconducting lead has on the magnetic molecule can be fully taken into account by introducing an effective Hamiltonian. The form of this effective Hamiltonian can be obtained by applying the diagrammatic perturbation theory of Chapter 2 to the coupling between the molecule and the superconducting lead. Physically this approximation means that all the electrons in the superconductor form Cooper pairs; when an electron leaves the superconductor to tunnel to the molecule the other electron in the Cooper pair also has to leave the condensate since there are no available single electron states. The order of the time separation between the two electrons in the pair leaving the superconductor is determined by $1 / \Delta$. Therefore as $\Delta \rightarrow \infty$ the time goes to zero. Because both electrons in a Cooper pair have to leave the superconductor at the same time, or two electrons must enter the superconductor at the same time, the interaction between the molecule and the superconductor must have the form $E_{1} d_{\uparrow}^{+} d_{\downarrow}^{+}+E_{2} d_{\downarrow} d_{\uparrow}$, where $E_{1 / 2}$ is some energy.

a)
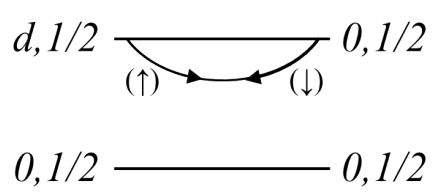

b)

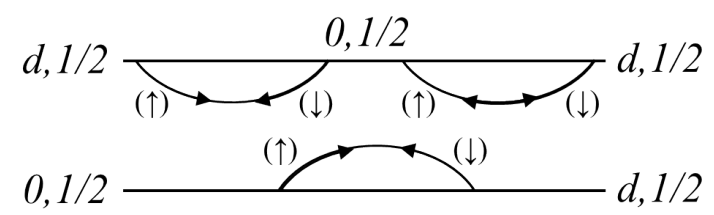

Figure 3.2: a) Diagrams of this form are non-zero and do not cancel out in the $\Delta \rightarrow \infty$ limit. b) Higher order diagrams of this form are zero in the $\Delta \rightarrow \infty$ limit.

We will now use the diagrammatic technique described in Chapter 2 to derive the exact form of the effective Hamiltonian. It can be shown that in the $\Delta \rightarrow \infty$ limit the only diagrams that are non-zero are first order diagrams connecting vertices on the same propagator where two electrons are either created or destroyed in the molecule [5]. Figure 3.2 a) shows the type of diagram that must be calculated. Higher 
order diagrams, such as the one shown in Fig. $3.2 \mathrm{~b}$ ), are proportional to $1 / \Delta$ and therefore tend to zero in the infinite band gap limit. Using the diagrammatic rules given in the Appendix $\mathrm{A}$ we get

$$
\begin{gathered}
W_{0,1 / 20,1 / 2}^{0,1 / 2 d, 1 / 2}=\frac{i \Gamma_{S}}{2 \pi} \int_{-\infty}^{\infty} f^{+}(\omega) \operatorname{sign}(\omega) \frac{|\Delta| \theta(|\omega|-|\Delta|)}{\sqrt{\omega^{2}-|\Delta|^{2}}} \\
\left(\frac{1}{\omega-E_{T+}+i 0^{+}}+\frac{1}{2\left(\omega-E_{T 0}+i 0^{+}\right)}+\frac{1}{2\left(\omega-E_{S}+i 0^{+}\right)}\right) d \omega
\end{gathered}
$$

where $E_{\chi}$ is the energy of state $\chi$ and $f^{+}(\omega)=\frac{1}{\mathrm{e}^{\beta\left(\omega-\mu_{S}\right)}+1}$ is the Fermi function. One of the diagrams for this generalised transition rate is that given in Fig. 3.2 a). Only transitions between states with the same molecular spin are non-zero, as there is no mechanism to change the molecular spin in the tunneling Hamiltonian. If $|\Delta|$ is very large then $f^{+}(\omega)=0$ when $\omega>|\Delta|$ and $f^{+}(\omega)=1$ when $\omega<-|\Delta|$. In this limit Eq. 3.14 becomes

$$
W_{0,1 / 2}^{0,1 / 2 d, 1 / 2}=\frac{-i \Gamma_{S}}{2 \pi} \int_{-\infty}^{-\Delta} \frac{|\Delta|}{\sqrt{\omega^{2}-|\Delta|^{2}}}\left(\frac{1}{\omega-E_{T+}+i 0^{+}}+\sum_{\eta=T 0, S} \frac{1}{2\left(\omega-E_{\eta}+i 0^{+}\right)}\right) d \omega .
$$

Introducing $x=\frac{-\omega}{\mid \Delta}$ we get

$$
W_{0,1 / 2}^{0,1 / 2 d, 1 / 2}=\frac{-i \Gamma_{S}}{2 \pi} \int_{1}^{\infty} \frac{1}{\sqrt{x^{2}-1}}\left(\frac{1}{-x-\frac{E_{T+}}{|\Delta|}+\frac{i 0^{+}}{|\Delta|}}+\sum_{\eta=T 0, S} \frac{1}{2\left(-x-\frac{E_{\eta}}{|\Delta|}+\frac{i 0^{+}}{|\Delta|}\right)}\right) d \omega
$$

In the limit of $\Delta \rightarrow \infty$ Eq. 3.16 becomes

$$
W_{0,1 / 2 d, 1 / 2}^{0,1 / 2 d, 1 / 2}=\frac{i \Gamma_{S}}{\pi} \int_{1}^{\infty} \frac{d \omega}{x \sqrt{x^{2}-1}}=\frac{i \Gamma_{S}}{2}
$$

Performing a similar calculation we find $W_{0,1 / 2 d, 1 / 2}^{0,1 / 2}=\frac{-i \Gamma_{S}}{2}$. Using Eq. 2.20 the time evolution of $P_{0,1 / 2}$ in the stationary limit is

$$
\frac{d P_{0,1 / 2}}{d t}=0=W_{0,1 / 2 ~ 0,1 / 2}^{0,1 / 2 d, 1 / 2} P_{0,1 / 2}^{d, 1 / 2}+W_{0,1 / 2 d, 1 / 2}^{0,1 / 2} P_{d, 1 / 2}^{0,1 / 2}=\frac{i \Gamma_{S}}{2}\left(P_{0,1 / 2}^{d, 1 / 2}-P_{d, 1 / 2}^{0,1 / 2}\right) .
$$

All other diagrams are also equal to $\pm \frac{i \Gamma_{S}}{2}$. We can deduce that the form of the effective Hamiltonian is

$$
H_{e f f}=H_{M}-\frac{\Gamma_{S}}{2}\left(d_{\uparrow}^{+} d_{\downarrow}^{+}+d_{\downarrow} d_{\uparrow}\right) .
$$

Using basis $\{|0,1 / 2\rangle,|d, 1 / 2\rangle,|T+\rangle,|T-\rangle,|T 0\rangle,|S\rangle,|0,-1 / 2\rangle,|d,-1 / 2\rangle\}, H_{\text {eff }}$ can be 
written as

$$
H_{e f f}=\left(\begin{array}{cccccccc}
0 & \frac{-\Gamma_{S}}{2} & 0 & 0 & 0 & 0 & 0 & 0 \\
\frac{-\Gamma_{S}}{2} & 2 \epsilon+U & 0 & 0 & 0 & 0 & 0 & 0 \\
0 & 0 & \epsilon+\frac{J}{4} & 0 & 0 & 0 & 0 & 0 \\
0 & 0 & 0 & \epsilon+\frac{J}{4} & 0 & 0 & 0 & 0 \\
0 & 0 & 0 & 0 & \epsilon+\frac{J}{4} & 0 & 0 & 0 \\
0 & 0 & 0 & 0 & 0 & \epsilon-\frac{3 J}{4} & 0 & 0 \\
0 & 0 & 0 & 0 & 0 & 0 & 0 & \frac{-\Gamma_{S}}{2} \\
0 & 0 & 0 & 0 & 0 & 0 & \frac{-\Gamma_{S}}{2} & 2 \epsilon+U
\end{array}\right)
$$

We can check that the dynamics of this system are described by this Hamiltonian by calculating the time evolution of the reduced density matrix using $\frac{d \rho^{M}}{d t}=0=i\left[\rho^{M}, H_{e f f}\right]$. The reduced density matrix for this system is

$$
\rho^{M}=\left(\begin{array}{cccccccc}
P_{0,1 / 2} & P_{d, 1 / 2}^{0,1 / 2} & 0 & 0 & 0 & 0 & 0 & 0 \\
P_{0,1 / 2}^{d, 1 / 2} & P_{d, 1 / 2} & 0 & 0 & 0 & 0 & 0 & 0 \\
0 & 0 & P_{T+} & 0 & 0 & 0 & 0 & 0 \\
0 & 0 & 0 & P_{T-} & 0 & 0 & 0 & 0 \\
0 & 0 & 0 & 0 & P_{T 0} & 0 & 0 & 0 \\
0 & 0 & 0 & 0 & 0 & P_{S} & 0 & 0 \\
0 & 0 & 0 & 0 & 0 & 0 & P_{0,-1 / 2} & P_{d,-1 / 2}^{0,-1 / 2} \\
0 & 0 & 0 & 0 & 0 & 0 & P_{0,-1 / 2}^{d,-1 / 2} & P_{d,-1 / 2}
\end{array}\right) .
$$

Therefore the commutator of $\rho^{M}$ and $H_{\text {eff }}$ is

$$
\left(\begin{array}{cc}
\frac{\Gamma_{S}}{2}\left(P_{0,1 / 2}^{d, 1 / 2}-P_{d, 1 / 2}^{0,1 / 2}\right) & \frac{\Gamma_{S}}{2}\left(P_{d, 1 / 2}-P_{0,1 / 2}\right)+E_{d, 1 / 2} P_{d, 1 / 2}^{0,1 / 2} \\
-\frac{\Gamma_{S}}{2}\left(P_{d, 1 / 2}-P_{0,1 / 2}\right)-E_{d, 1 / 2} P_{0,1 / 2}^{d, 1 / 2} & -\frac{\Gamma_{S}}{2}\left(P_{0,1 / 2}^{d, 1 / 2}-P_{d, 1 / 2}^{0,1 / 2}\right)
\end{array}\right)
$$

and

$$
\left(\begin{array}{cc}
\frac{\Gamma_{S}}{2}\left(P_{0,-1 / 2}^{d-, 1 / 2}-P_{d,-1 / 2}^{0,-1 / 2}\right) & \frac{\Gamma_{S}}{2}\left(P_{d,-1 / 2}-P_{0,-1 / 2}\right)+E_{d,-1 / 2} P_{d,-1 / 2}^{0,-1 / 2} \\
-\frac{\Gamma_{S}}{2}\left(P_{d,-1 / 2}-P_{0,-1 / 2}\right)-E_{d,-1 / 2} P_{0,-1 / 2}^{d,-1 / 2} & -\frac{\Gamma_{S}}{2}\left(P_{0,-1 / 2}^{d,-1 / 2}-P_{d,-1 / 2}^{0,-1 / 2}\right)
\end{array}\right)
$$

for the bases $\{|0,1 / 2\rangle,|d, 1 / 2\rangle\}$ and $\{|0,-1 / 2\rangle,|d,-1 / 2\rangle\}$ and zero otherwise. Giving $\frac{d P_{0,1 / 2}}{d t}=0=\frac{i \Gamma_{S}}{2}\left(P_{0,1 / 2}^{d, 1 / 2}-P_{d, 1 / 2}^{0,1 / 2}\right)$, the correct result for the time evolution of $P_{0}$. All $P_{\chi}^{\chi^{\prime}}$ can be checked likewise.

Because the effective Hamiltonian is block diagonal the eigenvalues and eigenvectors 
can easily be found. Four of the eigenstates are Andreev bound states given by

$$
|+, \pm\rangle=\frac{1}{\sqrt{2}} \sqrt{1-\frac{\delta}{2 \epsilon_{A}}}|0, \pm 1 / 2\rangle-\frac{1}{\sqrt{2}} \sqrt{1+\frac{\delta}{2 \epsilon_{A}}}|d, \pm 1 / 2\rangle
$$

and

$$
|-, \pm\rangle=\frac{1}{\sqrt{2}} \sqrt{1+\frac{\delta}{2 \epsilon_{A}}}|0, \pm 1 / 2\rangle+\frac{1}{\sqrt{2}} \sqrt{1-\frac{\delta}{2 \epsilon_{A}}}|d, \pm 1 / 2\rangle
$$

with energies

$$
E_{+}=\frac{\delta}{2}+\epsilon_{A}
$$

and

$$
E_{-}=\frac{\delta}{2}-\epsilon_{A},
$$

respectively. Here $\delta=2 \epsilon+U$ is the detuning and $2 \epsilon_{A}=\sqrt{\delta^{2}+\Gamma_{S}^{2}}$. The remaining four states form a triplet and a singlet state. The triplet states are given by

$$
\begin{gathered}
|T+\rangle=|\uparrow, 1 / 2\rangle, \\
|T-\rangle=|\downarrow,-1 / 2\rangle
\end{gathered}
$$

and

$$
|T 0\rangle=\frac{1}{\sqrt{2}}(|\downarrow, 1 / 2\rangle+|\uparrow,-1 / 2\rangle)
$$

with energy

$$
E_{T}=\epsilon+\frac{J}{4}
$$

And the singlet is given by

$$
|S\rangle=\frac{1}{\sqrt{2}}(|\downarrow, 1 / 2\rangle-|\uparrow,-1 / 2\rangle)
$$

with energy

$$
E_{S}=\epsilon-\frac{3 J}{4} .
$$

These four states are the same as those of the isolated molecule, as singly occupied states cannot couple to an infinite gap superconductor.

The Andreev bound states arise due to the coupling to superconducting lead. When this coupling is in resonance then the superposition of the empty and the doubly occupied states will be maximal. This occurs when $\Gamma_{S} \gg \delta$, in which case the bound states reduce to $|+, \pm\rangle=\frac{1}{\sqrt{2}}|0, \pm 1 / 2\rangle-\frac{1}{\sqrt{2}}|d, \pm 1 / 2\rangle$ and $|-, \pm\rangle=\frac{1}{\sqrt{2}}|0, \pm 1 / 2\rangle+\frac{1}{\sqrt{2}}|d, \pm 1 / 2\rangle$, 
as $\frac{\delta}{2 \epsilon_{A}} \approx \frac{\delta}{\Gamma_{S}} \approx 0$. The above condition is fulfilled for arbitrary coupling strengths when $\epsilon=\frac{-U}{2}$. When the superconducting proximity effect is not in resonance, $|\delta| \gg \Gamma_{S}$, then the states reduce to $|+, \pm\rangle=-|d, \pm 1 / 2\rangle$ and $|-, \pm\rangle=|0, \pm 1 / 2\rangle$ for positive $\delta$ and $|+, \pm\rangle=|0, \pm 1 / 2\rangle$ and $|-, \pm\rangle=|d, \pm 1 / 2\rangle$ for negative $\delta$.

The singlet and triplet states arise due to the coupling of the electron spin and the molecular spin. If the coupling constant tends to zero, $J \rightarrow 0$, then all four states are degenerate and the system reduces to two copies of a single level quantum dot coupled to a superconducting lead, one copy for the each of the molecular spins as there is no longer any superposition of states with different molecular spins.

Transitions between the eigenstates of $H_{\text {eff }}$ are induced by tunneling events involving the normal lead. For transition to occur the energy of the electron entering or leaving the normal lead must account for the energies difference between the initial and final states of the IMM-S subsystem. The excitation energies are therefore very important for understanding the transport properties of this system. To zeroth order in the coupling to the normal lead, the excitation energies are

$$
\begin{aligned}
& E_{T+}= \pm\left|E_{T}-E_{+}\right|= \pm\left|\frac{J}{4}-\frac{U}{2}-\epsilon_{A}\right|, \\
& E_{T-}= \pm\left|E_{T}-E_{-}\right|= \pm\left|\frac{J}{4}-\frac{U}{2}+\epsilon_{A}\right|, \\
& E_{S+}= \pm\left|E_{S}-E_{+}\right|= \pm\left|\frac{3 J}{4}+\frac{U}{2}+\epsilon_{A}\right|
\end{aligned}
$$

and

$$
E_{S-}= \pm\left|E_{S}-E_{-}\right|= \pm\left|\frac{3 J}{4}+\frac{U}{2}-\epsilon_{A}\right|
$$

\subsection{Transition Rates and Current}

To calculate the steady state current flowing through the system we must first calculate all the relevant generalised transition rates between the elements of the reduced density matrix of the IMM-S subsystem. In the most general case there could be off-diagonal density matrix elements that contribute to the dynamics of the system, in which case one would need to use the diagrammatic technique described in Chapter 2 to calculate the generalised transition rates to and from these off-diagonal elements. With an 8x8 density matrix this could mean having to calculate up to 4096 generalised transition rates. However, due to the regime we are working in, most of these rates are zero or 


$$
\begin{array}{|c|c|c|c|}
\hline|++\rangle \rightleftharpoons|T+\rangle & - & |++\rangle \rightleftharpoons|T 0\rangle & |++\rangle \rightleftharpoons|S\rangle \\
- & |+-\rangle \rightleftharpoons|T-\rangle & |+-\rangle \rightleftharpoons|T 0\rangle & |+-\rangle \rightleftharpoons|S\rangle \\
|-+\rangle \rightleftharpoons|T+\rangle & - & |-+\rangle \rightleftharpoons|T 0\rangle & |-+\rangle \rightleftharpoons|S\rangle \\
- & |--\rangle \rightleftharpoons|T-\rangle & |--\rangle \rightleftharpoons|T 0\rangle & |--\rangle \rightleftharpoons|S\rangle \\
\hline
\end{array}
$$

Table 3.1: Transitions relevant to first order transport through the N-IMM-S system.

not relevant to the transport properties of the system. Firstly, we are only interested in first order transitions, meaning that we do not consider transitions between Andreev bound states, or between the singlet-triplet states. Secondly, we are working in the regime where $\Gamma_{S} \gg \Gamma_{N}$. This means for off-diagonal elements involving one of the bound states Eq. 2.20 reduces to $0=-i\left(\epsilon_{\chi_{1}}-\epsilon_{\chi_{2}}\right) P_{\chi_{2}}^{\chi_{1}}$, as $\epsilon_{\chi_{1}}-\epsilon_{\chi_{2}}$ would be of the order of $\Gamma_{S}$ and $W_{\chi_{2} \chi_{2}^{\prime}}^{\chi_{1} \chi_{1}^{\prime}}$ only of the order of $\Gamma_{N}$. Therefore $P_{\chi_{2}}^{\chi_{1}}$ must be equal to zero. We cannot use the same reasoning to deduce that superpositions of the $| \pm+\rangle$ and $| \pm-\rangle$ states will be zero, however these can be neglected because they can only couple to superpositions of the singlet-triplet states and not to any diagonal density matrix elements. Therefore these superpositions do not contribute to the dynamics of the diagonal elements. Hence the only transitions that are relevant are those from the Andreev bound states to the singlet-triplet states and vice versa. Of these transitions the four in which the molecular spin is not conserved are also equal to zero. The transitions that are relevant to transport in this system are given in Table 3.1

Because the only transitions that effect the dynamics of this system are first order transitions between diagonal elements of the reduced density matrix, it is not necessary to use diagrammatic techniques to calculate the transition rates. Instead they can be calculated more simply using Fermi's golden rule -

$$
W_{f, i}=2 \pi \int\left|\left\langle f\left|H_{t u n n, N}\right| i\right\rangle\right|^{2} \delta\left(E_{i}-E_{f}\right) d \omega
$$

Here $W_{f, i}$ is the transition rate from the initial state $|i\rangle$ to the final state $|f\rangle, E_{i}$ and $E_{f}$ are the energies of the initial and final states and the integral is over all available states of the normal lead. The initial and final states involve the state of the molecule and the state of the lead. For example:

$$
|i\rangle=|T+\rangle_{\text {molecule }} \otimes|\omega\rangle_{\text {lead }} \rightarrow|f\rangle=|++\rangle_{\text {molecule }} \otimes|0\rangle_{\text {lead }}
$$


The lead states represent the Fermi sea plus a state at energy $\omega$, which is either occupied, $|\omega\rangle_{\text {lead }}$, or unoccupied, $|0\rangle_{\text {lead }}$. To change to an integral over all energies the integrand is multiplied by the density of states in the normal lead and the Fermi function, which weighs the probability of the states being occupied (or one minus the Fermi function for the probability of the state being unoccupied). Note that we have assumed the density of states in the normal lead to be constant for our energy range of interest. With these changes Eq. 3.38 becomes

$$
W_{f, i}=2 \pi N_{N} \int f^{ \pm}(\omega)\left|\left\langle f\left|H_{\text {tunn }, N}\right| i\right\rangle\right|^{2} \delta\left(E_{i}-E_{f}\right) d \omega
$$

where $f^{-}(\omega)=1-f^{+}(\omega)$.

Using Eq. 3.40, the non-zero transition rates are

$$
\begin{array}{r}
W_{ \pm+, T+}=W_{ \pm-, T-}=\frac{\Gamma_{N}}{2}\left[\delta_{\mp}^{2} f^{-}\left(E_{T}-E_{ \pm}\right)+\delta_{ \pm}^{2} f^{+}\left(E_{ \pm}-E_{T}\right)\right] \\
W_{T+, \pm+}=W_{T-, \pm-}=\frac{\Gamma_{N}}{2}\left[\delta_{\mp}^{2} f^{+}\left(E_{T}-E_{ \pm}\right)+\delta_{ \pm}^{2} f^{-}\left(E_{ \pm}-E_{T}\right)\right] \\
W_{ \pm+, \eta}=W_{ \pm-, \eta}=\frac{\Gamma_{N}}{4}\left[\delta_{\mp}^{2} f^{-}\left(E_{\eta}-E_{ \pm}\right)+\delta_{ \pm}^{2} f^{+}\left(E_{ \pm}-E_{\eta}\right)\right]
\end{array}
$$

and

$$
W_{\eta, \pm+}=W_{\eta, \pm-}=\frac{\Gamma_{N}}{4}\left[\delta_{\mp}^{2} f^{+}\left(E_{\eta}-E_{ \pm}\right)+\delta_{ \pm}^{2} f^{-}\left(E_{ \pm}-E_{\eta}\right)\right],
$$

where $\delta_{ \pm}^{2}=\left(1 \pm \frac{\delta}{2 \epsilon_{A}}\right)$ and $\eta=S, T 0$.

When Fermi's golden rule is used there are no transition rates of the form $W_{\chi \chi}$. This means that we must slightly modify Eq. 2.20 when using Fermi's golden rule. The modified equation for the steady state evolution of the reduced density matrix elements is

$$
0=\sum_{\chi^{\prime} \neq \chi}\left(W_{\chi \chi^{\prime}} P_{\chi^{\prime}}-W_{\chi^{\prime} \chi} P_{\chi}\right)
$$

For our system the master equation gives eight linear simultaneous equations. Along with the condition $\sum_{\chi} P_{\chi}=1$, these equations can be solved to find the occupation probabilities, $P_{\chi}$. To calculation the current we use Eq. 2.24. The first order current rates can easily be determined from the transition rates as the only change is a sign difference for electrons entering or leaving the molecule. All terms containing a $f^{-}(E)$ term are multiplied by -1 .

We also calculate the Fano factor using the method described at the end of Chapter 
2. To do this the counting field must be introduced into the transition rates, which is done by inserting the factor $\mathrm{e}^{ \pm i \xi}$ into each term, where the sign is positive if an electron is entering the normal lead and negative if an electron is leaving the normal lead.

\subsection{Results}

In this section we will analyse the results of the probability, current and Fano factor calculations. Due to the large dimension of the Hilbert space of the system we were unable to obtain any analytic results. We have therefore made plots of numerical results for varying parameter values. At this stage it is useful to summarise the regime in which the results are valid. The current is valid for $\Gamma_{N} \ll k_{B} T$ and will be given in units of $\Gamma_{N}$. The molecule is strongly coupled to the superconducting lead, $\Gamma_{S} \gg \Gamma_{N}$, and the superconducting gap is infinite. Also note that we have chosen $\mu_{S}=0$.

\subsubsection{Equilibrium and Zero Exchange Coupling Limits}

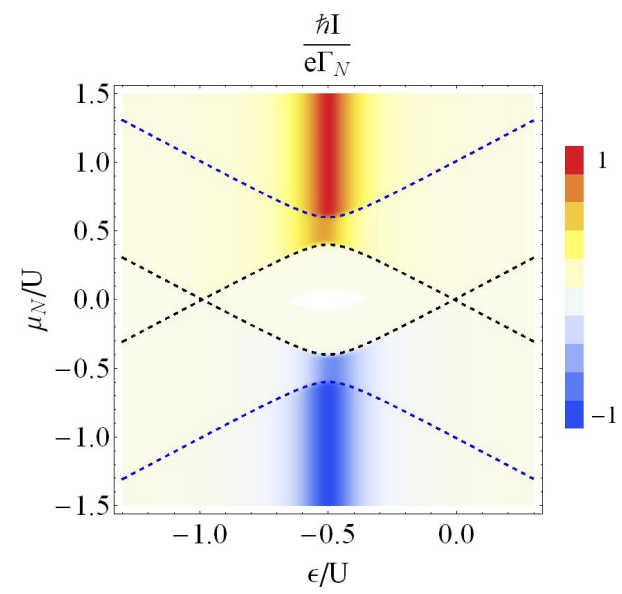

Figure 3.3: Density plot of the current as a function of the chemical potential $\mu_{N}$ and the level position $\epsilon$. The dashed lines show the excitation energies. The other parameters used in this plot are $\Gamma_{S}=0.2 U, \beta=100 / U$ and $J=0 U$.

Braggio et al. studied the transport properties of a quantum dot coupled to an infinite gap superconducting lead and a normal lead (N-QD-S). In the limit of $J \rightarrow 0$ the model for the N-IMM-S system reduces to that of the N-QD-S system. We find that our results agree with those of Braggio et al. This can be seen when comparing Fig. 3.3 with Fig. 2 of Ref. [47]. As a second consistency check we compare our results 
at equilibrium, $\mu_{S}=\mu_{N}=0$, to those obtained using Boltzmann statistics. We find that when $\mu_{N}=0$ the current is zero and the probabilities are in agreement with $P_{\chi}=\frac{e^{-\beta E_{\chi}}}{Z}$, where $Z=\sum_{\chi} e^{-\beta E_{\chi}}$.

\subsubsection{Andreev Current}
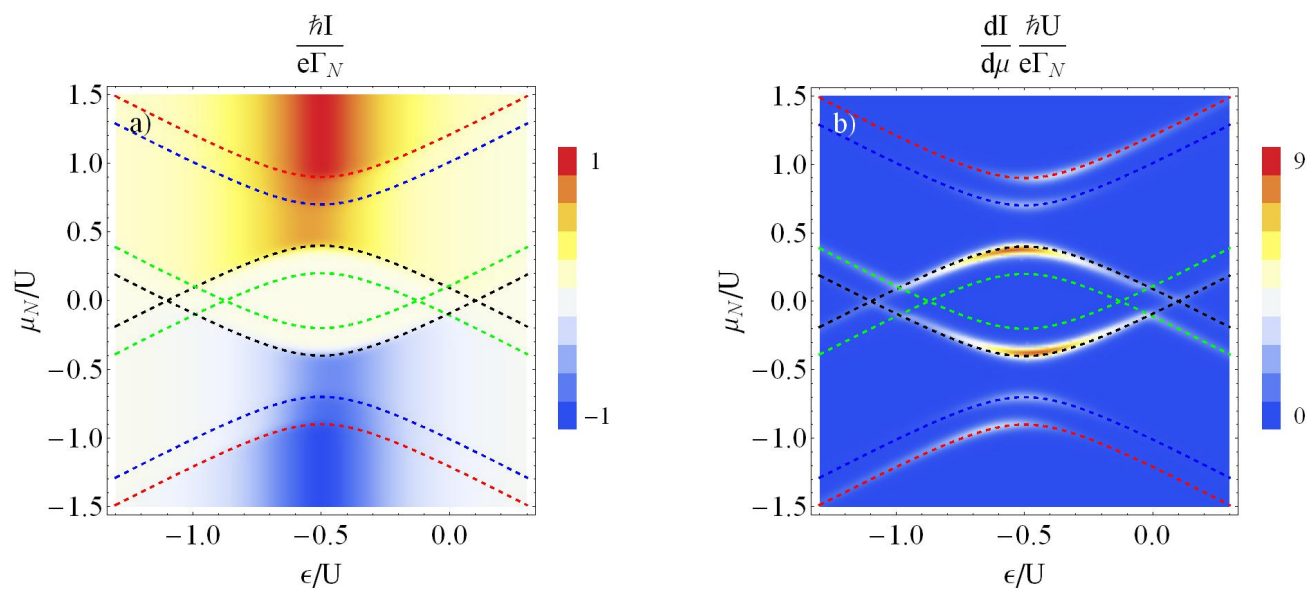

Figure 3.4: Density plots of the current (a) and the differential conductance (b) as functions of the chemical potential $\mu_{N}$ and the level position $\epsilon$. The dashed lines show the excitation energies. The red lines show $E_{S+}$, the black lines show $E_{S_{-}}$, the blue lines show $E_{T+}$ and the green lines show $E_{T-}$. The other parameters used in these plots are $\Gamma_{S}=0.5 U, \beta=50 / U$ and $J=0.2 U$.

To analyse the results for the current we have made density plots of the current, the differential conductance and the occupation probabilities as functions of the level position, $\epsilon$, and the chemical potential of the normal lead, $\mu_{N}$. Figure 3.4 shows such plots for the current and differential conductance. Here we can see that for any level position the maximum current is achieved when the chemical potential is greater than all the excitation energies, as under this condition all the states of the system can contribute to transport. The current is greatest when $\epsilon \approx-\frac{U}{2}$. This is because when this condition is met the superconducting proximity effect is in resonance as $\delta=0$ and therefore the superposition of the empty and doubly occupied states is maximal. Greater superposition of these states decreases waiting times between tunneling events to and from the superconducting lead, thus increasing the current. Either side of $\epsilon=-$ $0.5 U$ the current decreases, as the proximity effect goes out of resonance. Another notable feature is the central region where the current is zero. Plotted in Fig. 3.5 are the energies of the eigenstates of the molecule. For $\epsilon$ between about $-1.1 U$ and 
$0.1 U|S\rangle$ is the ground states of the molecule. This means in the central zero current region the molecule is trapped in the $|S\rangle$ state until the applied bias is high enough that transitions to the Andreev bound states can occur. Figure 3.6 shows the corresponding occupation probability plots and we see that in the central region the probability for $|S\rangle$ is one.

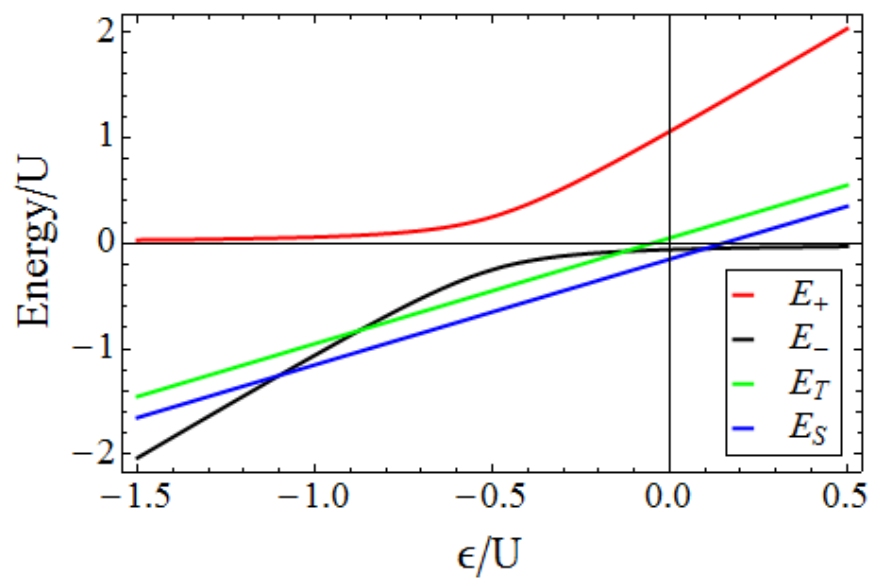

Figure 3.5: Energies of the eigenstates of the effective Hamiltonian as a function of the level position $\epsilon$. The other parameters used in this plot are $\Gamma_{S}=0.5 U$ and $J=0.2 U$.

Features in the current density plot in Fig. 3.4 occur along the excitation energies. This is more apparent when plotting the differential conductance, which is shown in Fig. 3.4 b). Here we see that changes in the current, with respect to $\mu_{N}$, occur only in the vicinity of the excitation energies. We also see that features do not occur along all parts of the excitation energies. This means that increasing $\left|\mu_{N}\right|$ from zero there is a stepwise increase in the current where an excitation energy is crossed, depending on the $\epsilon$ value. How sharp these steps are is determined by the temperature, as is shown in Fig. 3.7. Plots a) and b) of Fig. 3.7 show the current as a function of $\mu_{N}$ for $k_{B} T=0.05 U$ and $k_{B} T=0.01 U$, at the lower temperature we see very distinct steps in the current that occur at the excitation energies. This stepwise increase in the current occurs because the energies of the states in the normal lead need to be comparable to the energy difference of the states of the molecule. When $\mu_{N}$ is close to zero the molecule will remain in the ground state until $\mu_{N}$ is comparable to the energy difference to the next highest state, in which case an electron can tunnel to or from the normal lead. Because the superconducting lead only talks to the $|+ \pm\rangle$ and $|- \pm\rangle$ states there is no current when the molecule is trapped in the singlet state. However, as soon as $\mu_{N}$ is sufficiently high that transitions to $|- \pm\rangle$ can occur, current can flow. 

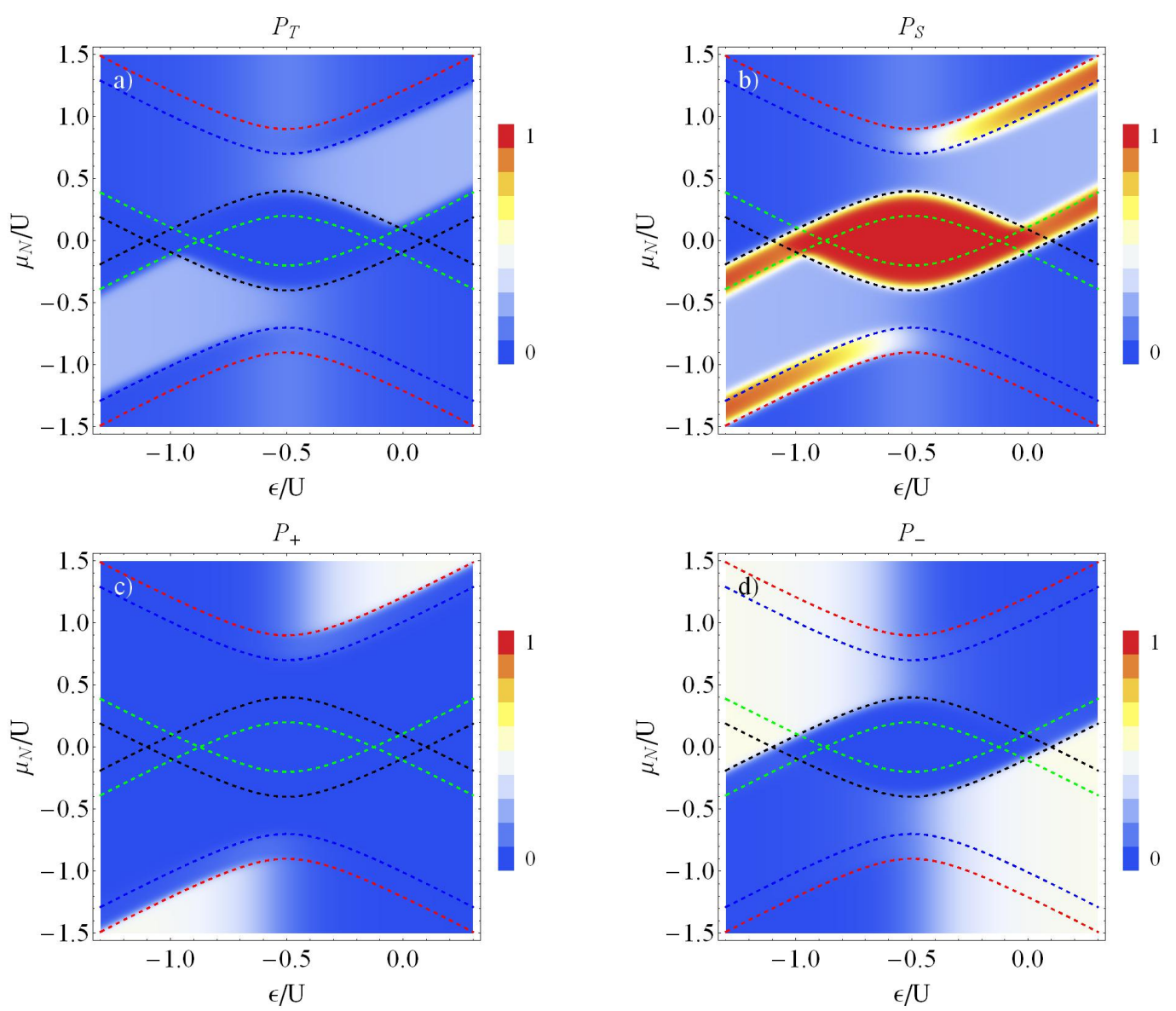

Figure 3.6: Density plots of the occupation probabilities for (a) the triplet, (b) the singlet and (c) (d) the Andreev bound states as functions of the chemical potential $\mu_{N}$ and the level position $\epsilon$. The probabilities for the three triplet states are equal, the same is true for the $|+, \pm\rangle$ states and the $|-, \pm\rangle$ states. The other parameters used in these plots are $\Gamma_{S}=0.5 U, \beta=50 / U$ and $J=0.2 U$. The dashed lines are the excitation energies as in Fig. 3.4.

To understand why features do not occur along all parts of the excitation energies we look at Fig. 3.6 showing the occupation probabilities. Features in the differential conductance plot only occur along parts of the excitation energies where there is a finite occupation probability of the two states involved. Comparing the probability plots to the current plot in Fig. 3.4 we see that the regions of highest current correspond to parameters values for which the probabilities are all comparable. This suggests that in these regions all states are equally contributing to the current. Plots c) and d) show regions of high probability for the bound states when the superconducting proximity effect is off resonance. For $\delta<0 \quad \delta_{+}<\delta_{-}$meaning that the $|+ \pm\rangle$states are weighted 

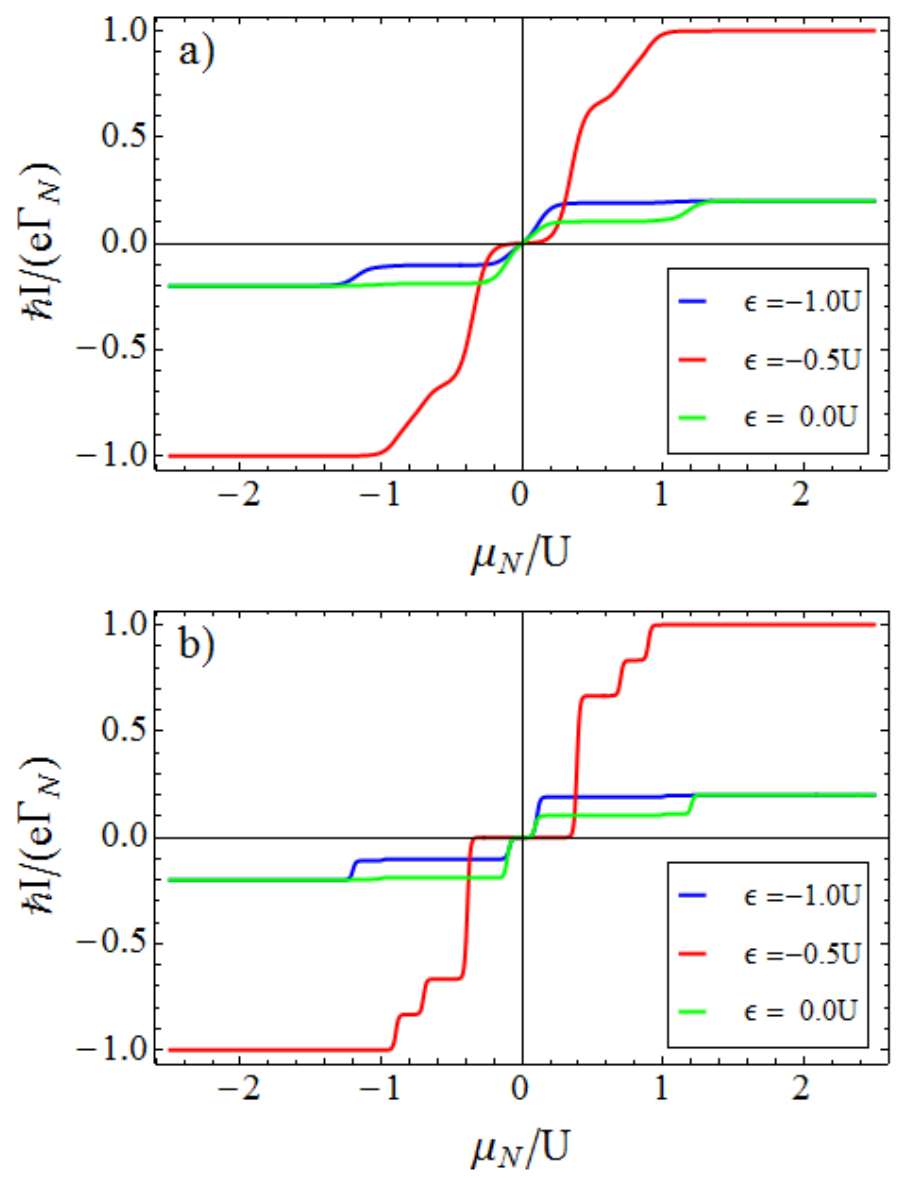

Figure 3.7: Plots of the current as a function of the chemical potential $\mu_{N}$ for (a) $k_{B} T=0.05 U$ and (b) $k_{B} T=0.01 U$. The other parameters used in these plots are $\Gamma_{S}=0.5 U$ and $J=0.2 U$.

more for the zero occupation and for $\delta>0 \quad \delta_{+}>\delta_{-}$meaning double occupation is more likely. The opposite is true for the $|- \pm\rangle$ states. Because the probabilities of all the degenerate eigenstates are the same, and in each set of degenerate eigenstates there are states of the molecule with opposite electronic and molecular spins that contribute equally, the expectation values of the electronic and molecular spins are zero for all parameter values.

Figure 3.8 shows the current for weak and for strong coupling to the superconducting lead. For weak coupling there is only a narrow range about $\delta=0$ where the current is non-zero, whereas for strong coulping this range is much wider. To understand this we look at the constants in the Andreev bound states, $\delta_{ \pm}=\sqrt{1 \pm \frac{\delta}{2 \epsilon_{A}}}$. When $\Gamma_{S}$ is sufficiently larger than $\delta$, then $\delta_{+} \approx \delta_{-}$and the superconducting proximtiy effect is 

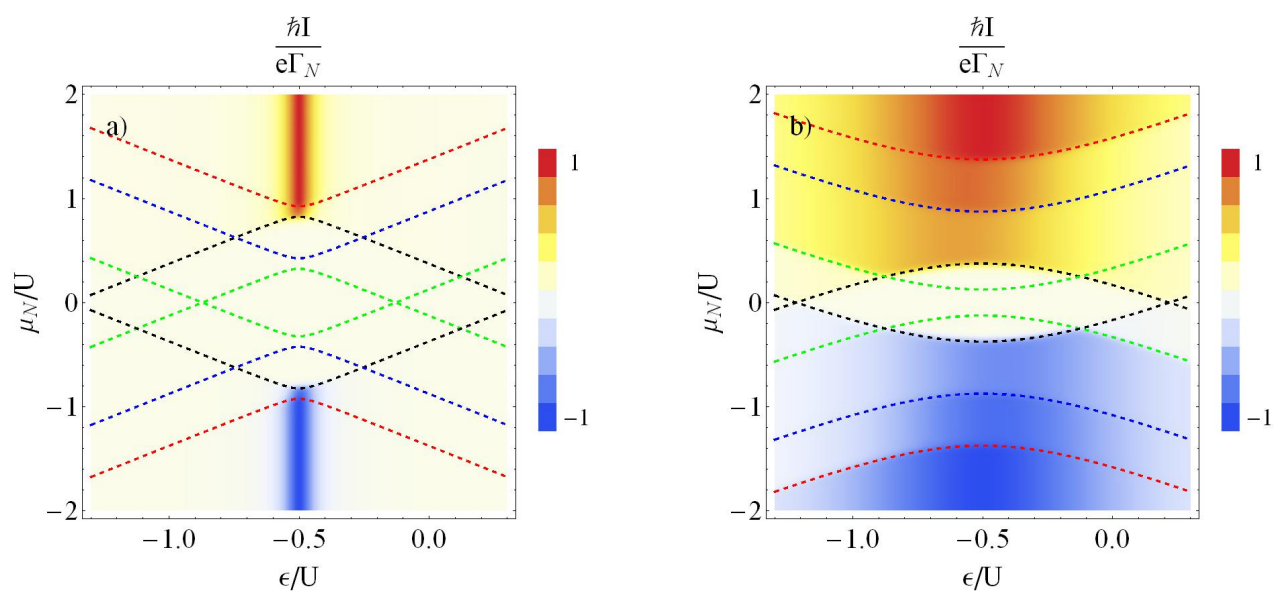

Figure 3.8: Density plots of the current as a function of the chemical potential $\mu_{N}$ and the level position $\epsilon$ for (a) weak, $\Gamma_{S}=0.1 U$, and (b) strong, $\Gamma_{S}=1 U$, coupling to the superconducting lead. The other parameters used in these plots are $\beta=50 / U$ and $J=0.5 U$. The excitation energies are shown as in Fig. 3.4.

in resonance, which leads to a large current. This occurs over a larger range of level position values when the coupling is stronger. Varying $\Gamma_{S}$ also affects the excitation energies and therefore the size of the central region where the current is zero. In Fig. 3.9 the current is plotted against $\epsilon$ at sufficiently high bias that the maximum current is reached. This is done for three superconductor coupling strengths. Here we see that the maximum current is independent of $\Gamma_{S}$ and confirm that width of the resonant current peak decreases with $\Gamma_{S}$. We also find that the width of the peaks at half maximum is equal to $\Gamma_{S}$.

Varying the strength of the exchange coupling affects the excitation energies. When $J$ is increased the size of the central region between the $E_{S-}$ excitation energies, where transport is suppressed, also increases. In Fig. 3.10 a) the differential conductance is plotted for $J=0.8 U$. We see that the $E_{T+}$ excitation line now crosses the $E_{S-}$ excitation line and that there are now no resonances along $E_{T+}$. In Fig. 3.10 b) we show cuts of the density plot along $\epsilon=-0.5 U$ for four values of the exchange coupling constant. In the latter we see a transition from two steps in the current to three and then to two again as $J$ is increased. For the smallest coupling there are only two steps discernible as the energy difference between the singlet and triplet states is very small. Then for intermediate values there are three steps and finally for the largest value there two steps. When the $E_{S-}$ and $E_{T+}$ excitation lines cross there are no differential conductance resonances at the excitation energies within the current suppressed region 


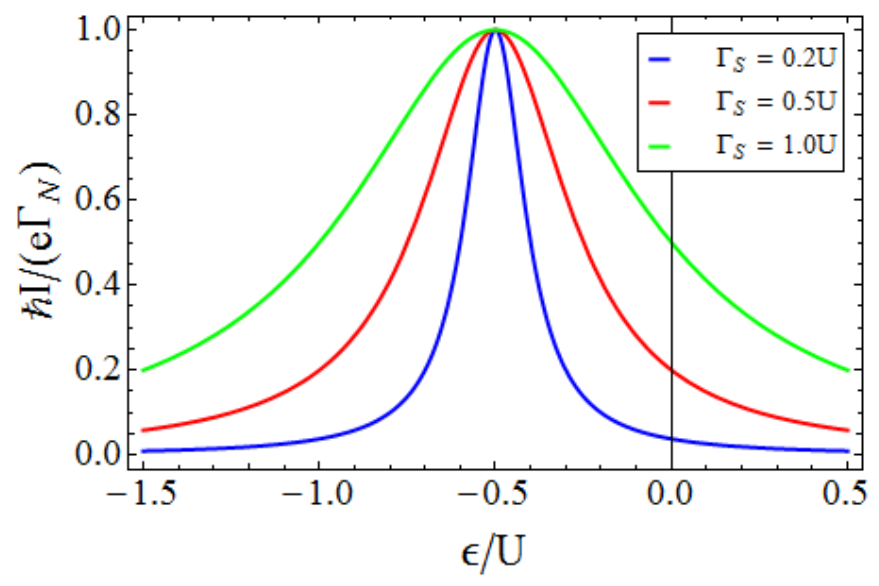

Figure 3.9: Plot of the current, at high bias, as a function of level position $\epsilon$ for three coupling strengths $\Gamma_{S}$. The other parameters used in this plot are $J=0.2 U, \beta=50 / U$ and $\mu_{N}=2 U$.

as here the molecule is in the singlet state and therefore no transitions involving the triplet states can occur. However as soon as the chemical potential of the normal lead is large enough that transitions out of the singlet state can occur, transport channels involving the triplet states are also opened. This is evident in the size of the first current step for $J=0.8 U$ in Fig. 3.10 b). Similarly for $J=0.1 U$ the second current step plateaus at the maximum current, as in this step all transitions to $|+ \pm\rangle$ are made possible. At high bias we find that the width of the current peaks are unaffected by the strength of the exchange coupling, meaning that $J$ has no affect on the superconducting proximity effect. This is because the exchange coupling only affects the states of the molecule that are singly occupied, whereas only the empty and doubly occupied states are affected by the proximity to the superconducting lead.

\subsubsection{Fano Factor}

It was found by Braggio et al. that for a single level dot, between a normal and a superconducting lead, the Fano factor is equal to 2 when the superconducting proximity effect is out of resonance and 1 when it is in resonance [47]. Setting $J$ equal to zero we find the same result (Fig. 3.11). Switching on the exchange coupling we find that the same behaviour occurs and that at high bias the Fano factor is not affected by the strength of the exchange coupling. 

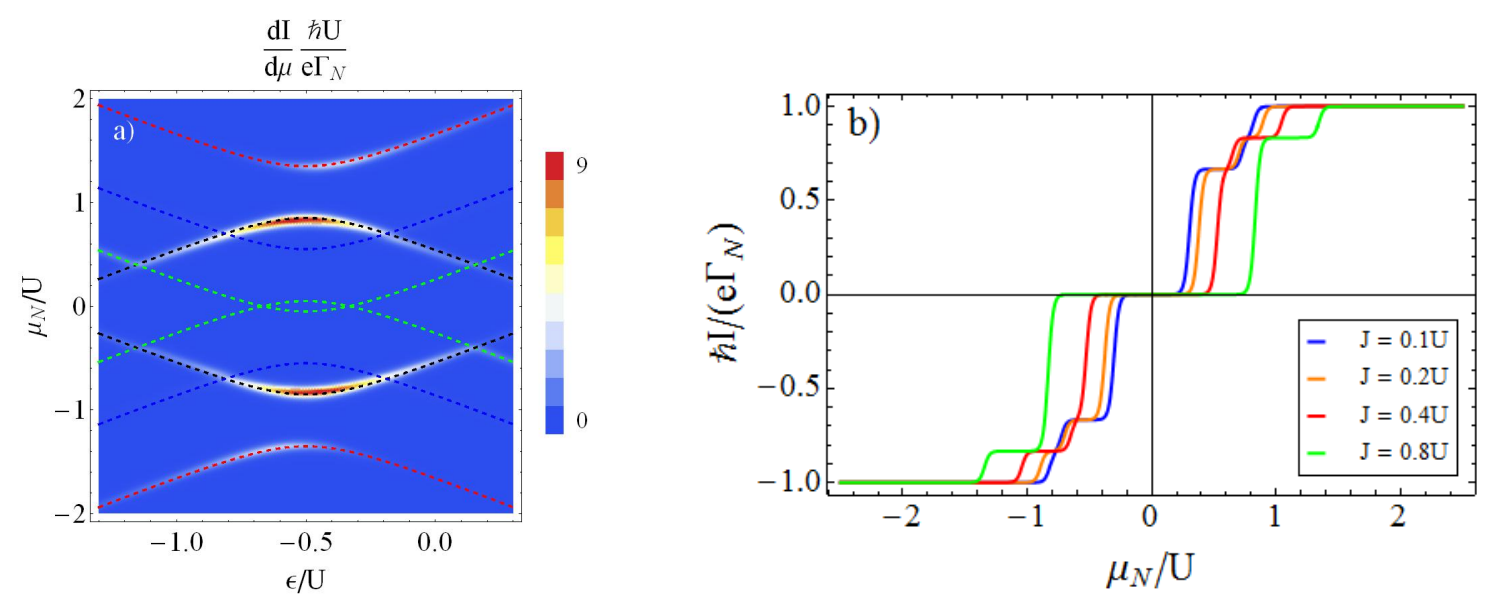

Figure 3.10: (a) Density plot of the differential conductance as a function of the chemical potential $\mu_{N}$ and the level position $\epsilon$ for $J=0.8 U$. (b) Plot of the current as a function of the chemical potential $\mu_{N}$ for three $J$ values, at $\epsilon=-0.5 U$. The other parameters used in these plots are $\Gamma_{S}=0.5 U$ and $\beta=50 / U$. The excitation energies are shown as in Fig. 3.4.

\section{$3.4 \quad$ N-IMM-S Conclusions}

In this chapter we presented a theoretical model for a single orbital isotropic magnetic molecule coupled to a normal lead and an infinite gap superconducting lead. We then calculated the reduced density matrix elements of the IMM-S subsystem, as well as the sequential current and the Fano factor. We found that the current is greatest when the superconducting proximity effect is in resonance and reduces to zero as the proximity effect goes out of resonance. Because only sub-gap transport to the superconducting lead is permitted and tunneling events to and from the normal lead, involving spin up and spin down electrons, are equally probable, the occupation probabilities of the eigenstates with the same energies are equal. This leads to the spin expectation values being equal to zero. Therefore, the magnetic molecule does not add any spin dependence to the transport in this system. In the current and differential conductance plots features occur along the excitation energies. Thus by measuring the current at varying bias and gate voltages a spectroscopy of the excitation energies could be performed. This would give information on the Andreev bound states and the exchange coupling between the electronic and molecular spins. Lastly, we found that Fano factor exhibits the same behaviour as a the N-QD-S system. It is equal to 1 when the superconducting proximity effect is in resonance, indicating Poissonian transport of single electrons, and out of resonance it is equal to 2, indicating Poissonian transport of electrons pairs. 


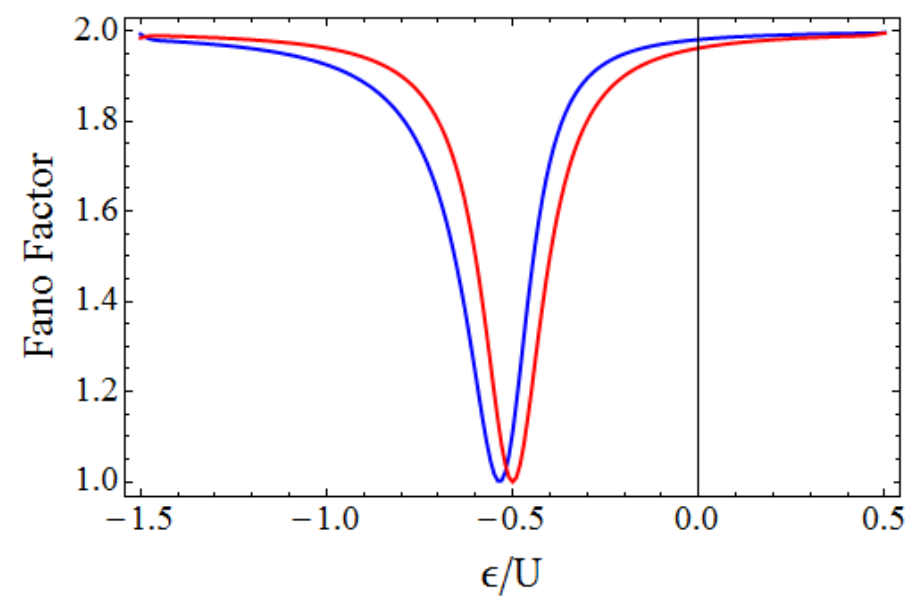

Figure 3.11: Plot of the Fano factor as a function of the level position $\epsilon$ for $\mu_{N}=0.5 U$ in blue and $\mu_{N}=1.5 U$ in red. The other parameters used in this plot are $\Gamma_{S}=0.2 U$, $\beta=100 / U$ and $J=0 U$. 


\section{Chapter 4}

\section{Anisotropic Magnetic Molecule Coupled to Normal and BCS Leads}

In the previous chapter we studied an isotropic magnetic molecule. However some realistic magnetic molecules, such as $\mathrm{Mn}_{12}$ [25], are anisotropic, therefore in this chapter we will analyse the current through an anisotropic magnetic molecule (AMM). All calculations in this chapter will be performed in the same way as in the previous chapter. In the anisotropic model we will include a quantum tunneling of magnetisation term and an anisotropy term.

\subsection{N-AMM-S System}

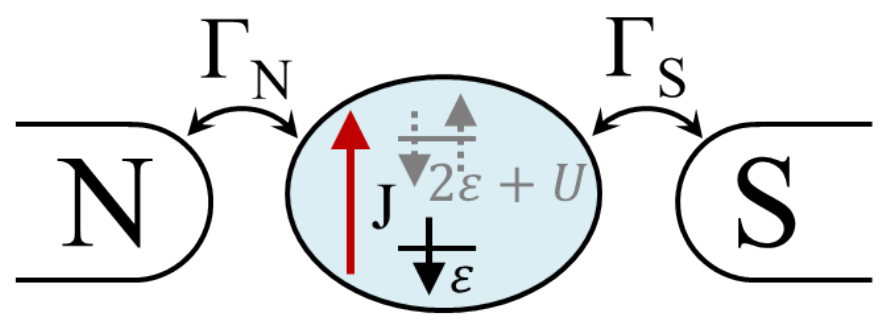

Figure 4.1: A single level anisotropic magnetic molecule coupled to a superconducting lead and a normal lead, with coupling strengths $\Gamma_{S}$ and $\Gamma_{N}$, and exchange coupling, $J$, between the spin of the electrons occupying the orbital level and the spin of the rest of the molecule.

To model an anisotropic magnetic molecule we add an anisotropy term, $D$, and 
a quantum tunneling of magnetisation term, $M$, to the Hamiltonian of the magnetic molecule to give

$$
H_{M}=\sum_{\sigma} \epsilon d_{\sigma}^{+} d_{\sigma}+U n_{\uparrow} n_{\downarrow}-D S_{Z}^{2}+\frac{M}{2}\left(S_{+}^{2}+S_{-}^{2}\right)+J \mathbf{S} . \mathbf{s}_{\mathbf{e}}
$$

where $S_{+}$and $S_{-}$are the ladder operators for the molecular spin. All other terms have the same definitions as in the previous chapter. The lead and tunneling Hamiltonians are the same as in Chapter 3 (Eqs. 3.12 and 3.13). The QTM term couples states with different molecular spins, as depicted in Fig. 4.2. We have only included the lowest order QTM term as higher order terms $\left(\frac{M_{n}}{2}\left(S_{+}^{2 n}+S_{-}^{2 n}\right), n=2,3, \ldots\right)$ are usually small [21].

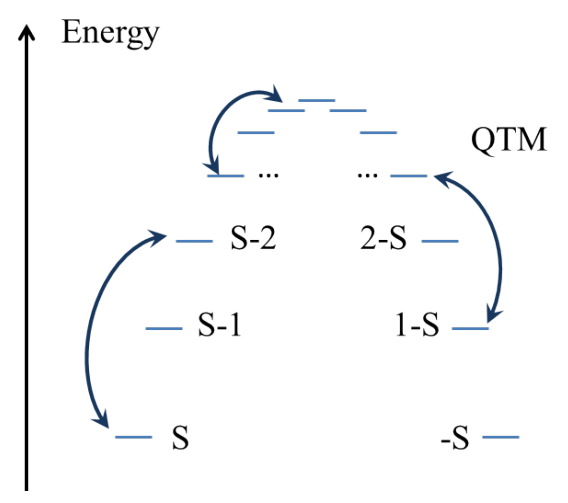

Figure 4.2: Quantum tunneling of magnetisation allows coupling between molecular spin states two levels apart. For $\mathrm{S}=1$ QTM couples the $|1\rangle_{M}$ and $|-1\rangle_{M}$ states.

The effect of the superconducting lead, to all orders in tunnel coupling, is again taken into account by using an effective Hamiltonian to describe MM-S subsytem. This effective Hamiltonian is now given by

$$
H_{e f f}=\sum_{\sigma} \epsilon d_{\sigma}^{+} d_{\sigma}+U n_{\uparrow} n_{\downarrow}-D S_{Z}^{2}+\frac{M}{2}\left(S_{+}^{2}+S_{-}^{2}\right)+J \mathbf{S} . \mathbf{s}_{\mathbf{e}}-\frac{\Gamma_{S}}{2}\left(d_{\uparrow}^{+} d_{\downarrow}^{+}+d_{\downarrow} d_{\uparrow}\right) .
$$

The coupling to the superconducting lead gives rise to the same term in the effective Hamiltonian as in the previous chapter. This is because the superconducting proximity effect only causes superpositions of $|0\rangle_{e}$ and $|d\rangle_{e}$ states that have the same molecular spin.

For simplicity we will use the smallest molecular spin, $S=1$, that will show the effects of the anisotropy and QTM. With $S=1$ there are now twelve states in this system; $|0,0\rangle,|d, 0\rangle,|0,-1\rangle,|d,-1\rangle,|0,1\rangle,|d, 1\rangle,|\uparrow, 0\rangle,|\downarrow, 1\rangle,|\downarrow,-1\rangle,|\downarrow, 0\rangle,|\uparrow,-1\rangle$, 
$|\uparrow, 1\rangle$. The effective Hamiltonian is block diagonal and the sub-matrices are given by

$$
\begin{gathered}
H_{A}=\left(\begin{array}{cc}
0 & \frac{-\Gamma_{S}}{2} \\
\frac{-\Gamma_{S}}{2} & 2 \epsilon+U
\end{array}\right) \\
H_{B}=\left(\begin{array}{cccc}
-D & \frac{-\Gamma_{S}}{2} & -M & 0 \\
\frac{-\Gamma_{S}}{2} & 2 \epsilon+U-D & 0 & -M \\
-M & 0 & -D & \frac{-\Gamma_{S}}{2} \\
0 & -M & \frac{-\Gamma_{S}}{2} & 2 \epsilon+U-D
\end{array}\right)
\end{gathered}
$$

and

$$
H_{C / D}=\left(\begin{array}{ccc}
\epsilon & \frac{J}{\sqrt{2}} & 0 \\
\frac{J}{\sqrt{2}} & \epsilon-D-\frac{J}{2} & -M \\
0 & -M & \epsilon-D+\frac{J}{2}
\end{array}\right)
$$

with the bases $\{|0,0\rangle,|d, 0\rangle\},\{|0,-1\rangle,|d,-1\rangle,|0,1\rangle,|d, 1\rangle\},\{|\uparrow, 0\rangle,|\downarrow, 1\rangle,|\downarrow,-1\rangle\}$ and $\{|\downarrow, 0\rangle,|\uparrow,-1\rangle,|\uparrow, 1\rangle\}$ for $H_{A}, H_{B}, H_{C}$ and $H_{D}$, respectively. Diagonalising these matrices leads to eigenstates of the form

$$
\begin{gathered}
|A\rangle=X 1_{A}|0,0\rangle+X 2_{A}|d, 0\rangle, \\
|B\rangle=X 1_{B}|0,-1\rangle+X 2_{B}|d,-1\rangle+X 3_{B}|0,1\rangle+X 4_{B}|d, 1\rangle, \\
|C\rangle=X 1_{C}|\uparrow, 0\rangle+X 2_{C}|\downarrow, 1\rangle+X 3_{C}|\downarrow,-1\rangle
\end{gathered}
$$

and

$$
|D\rangle=X 1_{D}|\downarrow, 0\rangle+X 2_{D}|\uparrow,-1\rangle+X 3_{D}|\uparrow, 1\rangle,
$$

where $A \rightarrow A 1, A 2, B \rightarrow B 1, B 2, B 3, B 4, C \rightarrow C 1, C 2, C 3$ and $D \rightarrow D 1, D 2, D 3$. The corresponding energies are given in Table 4.1 and the expressions for the eigenstate coefficients can be found in the Appendix B.

The sub-matrix $H_{A}$ produces two Andreev bound states similar to those encountered in the previous model. If we were considering an isotropic molecule then there would be four further Andreev bound states of the same form, two each for $S=1$ and $S=-1$. However due to the tunneling of magnetisation term in this model there is coupling between states with $S=1$ and $S=-1$. This leads to the form of the bound states that arise from the diagonalisation of the matrix $H_{B}$. The energies of these states are very similar to the Andreev bound state energies of $H_{A}$, however they have an extra term for the anisotropy and the tunneling of magnetisation. The form of the remaining six states arise due to the coupling of the electronic and molecular spins, as 


\begin{tabular}{|c||c|c|c|}
\hline$\chi$ & $A$ & $B$ & $C / D$ \\
\hline \hline$E_{\chi_{1}}$ & $\frac{\delta}{2}+\epsilon_{A}$ & $\frac{\delta}{2}-\epsilon_{A}-M-D$ & $\operatorname{Re}\left[\frac{\gamma+\beta^{2}+i \sqrt{3\left(\gamma-\beta^{2}\right)^{2}}}{12 \beta}\right]+\frac{3 \epsilon-2 D}{3}$ \\
$E_{\chi_{2}}$ & $\frac{\delta}{2}-\epsilon_{A}$ & $\frac{\delta}{2}-\epsilon_{A}+M-D$ & $\operatorname{Re}\left[\frac{\gamma+\beta^{2}-i \sqrt{3\left(\beta^{2}-\gamma\right)^{2}}}{12 \beta}\right]+\frac{3 \epsilon-2 D}{3}$ \\
$E_{\chi_{3}}$ & - & $\frac{\delta}{2}+\epsilon_{A}-M-D$ & $\operatorname{Re}\left[-\frac{\gamma+\beta^{2}}{6 \beta}\right]+\frac{3 \epsilon-2 D}{3}$ \\
$E_{\chi_{4}}$ & - & $\frac{\delta}{2}+\epsilon_{A}+M-D$ & - \\
\hline
\end{tabular}

Table 4.1: Energies of the 12 eigenstates of the anisotropic system. $\delta$ and $\epsilon_{A}$ are defined as in Chapter 3. The other terms are defined as $\beta=\left(\sqrt{\alpha}+72 M^{2} D-8 D^{3}+27 J^{3}\right)^{1 / 3}$, $\gamma=12 M^{2}+4 D^{2}+9 J^{2}$ and $\alpha=-\left(12 M^{2}+4 D^{2}+9 J^{2}\right)^{3}+\left(72 M^{2} D-8 D^{3}+27 J^{3}\right)^{2}$.

well as the tunneling of magnetisation. The $X 1_{\chi}|\sigma, 0\rangle+X 2_{\chi}|\bar{\sigma}, \pm 1\rangle$ terms are similar to the singlet and $|T 0\rangle$ states of the previous model.

\subsection{Transition Rates and Current}

Because we are still only interested in transport to first order in $\Gamma_{N}$, and there are no off-diagonal reduced density matrix elements that contribute to the dynamics of the system, we can once again use Fermi's golden rule to calculate the transition rates. The transitions that are made possible by first order tunneling events are $|\chi\rangle \rightleftharpoons\left|\chi^{\prime}\right\rangle$, with $\chi=A, B$ and $\chi^{\prime}=C, D$. Calculating the corresponding generalised transition rates we get

$$
\begin{gathered}
W_{A, C / C, A}=\Gamma_{N}\left|X 1_{C}\right|^{2}\left[\left|X 1_{A}\right|^{2} f^{-/+}\left(E_{C}-E_{A}\right)+\left|X 2_{A}\right|^{2} f^{+/-}\left(E_{A}-E_{C}\right)\right] \\
W_{A, D / D, A}=\Gamma_{N}\left|X 1_{D}\right|^{2}\left[\left|X 1_{A}\right|^{2} f^{-/+}\left(E_{D}-E_{A}\right)+\left|X 2_{A}\right|^{2} f^{+/-}\left(E_{A}-E_{D}\right)\right] \\
W_{B, C / C, B}=\Gamma_{N}\left[\left|X 1_{B} X 3_{C}+X 3_{B} X 2_{C}\right|^{2} f^{-/+}\left(E_{C}-E_{B}\right)\right. \\
\left.+\left|X 2_{B} X 3_{C}+X 4_{B} X 2_{C}\right|^{2} f^{+/-}\left(E_{B}-E_{C}\right)\right]
\end{gathered}
$$

and

$$
\begin{aligned}
W_{B, D / D, B}= & \Gamma_{N}\left[\left|X 1_{B} X 2_{D}+X 3_{B} X 3_{D}\right|^{2} f^{-/+}\left(E_{D}-E_{B}\right)\right. \\
& \left.+\left|X 2_{B} X 2_{D}+X 4_{B} X 3_{D}\right|^{2} f^{+/-}\left(E_{B}-E_{D}\right)\right]
\end{aligned}
$$


Using these rates we calculate the current in the same way as in Chapter 3. For this system we are not able to obtain analytic results. Therefore in the next section we will analyse numerical results, as was done in Chapter 3.

\subsection{Results}
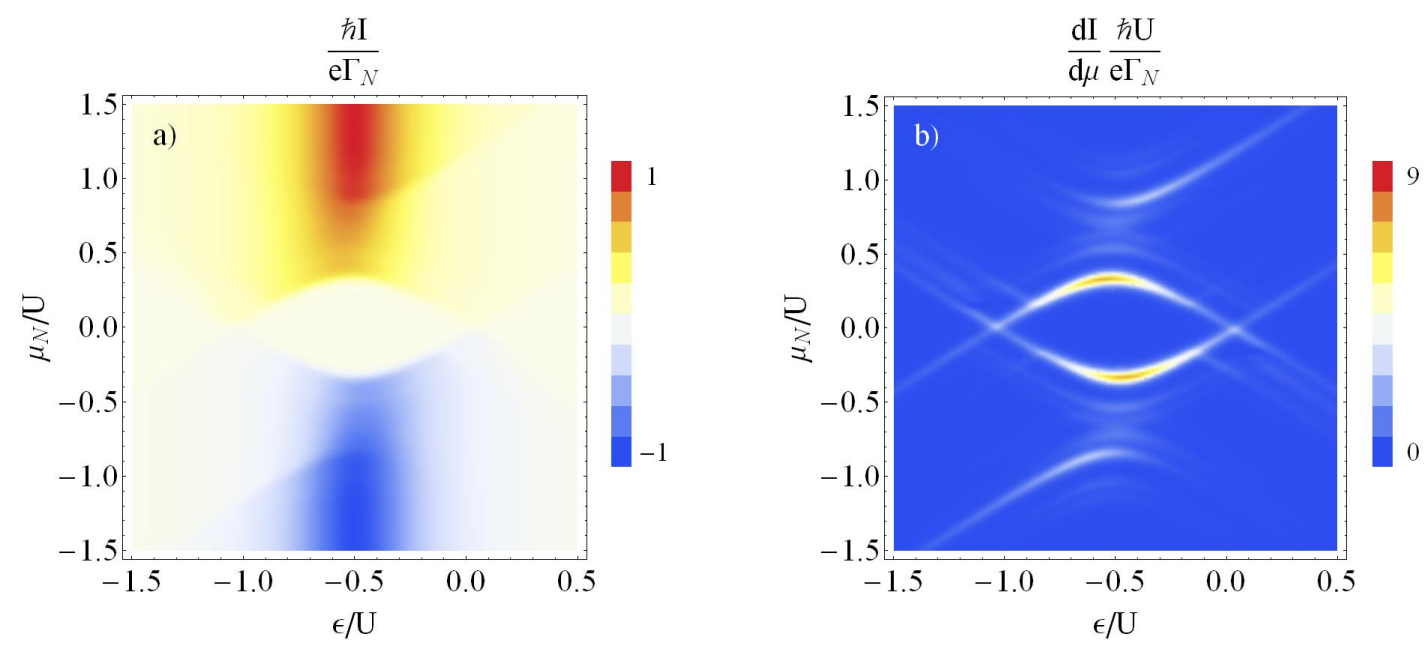

Figure 4.3: Density plots of the current (a) and the differential conductance (b) as functions of the chemical potential $\mu_{N}$ and the level position $\epsilon$. The other parameters used in these plots are $\Gamma_{S}=0.5 U, k_{B} T=0.02 U, J=0.2 U, D=0.2 U$ and $M=0.1 U$.

Figure 4.3 shows an example of the current and differential conductance of the N-AMM-S system. These plots are very similar to those of the N-IMM-S system analysed in Chapter 3, however we see more features due to the larger Hilbert space of this system. In the limit of $J$ tending to zero the dynamics of the anisotropic magnetic molecule reduce to those of a single level quantum dot. The anisotropy and the QTM have no affect in this limit as it is only through the exchange coupling between the electronic and molecular spins that the molecular spin influences the dynamics of the system. The effect that the strength of the exchange coupling has on the current is the same as in the previous model. As $J$ is increased the central low conduction region becomes larger and some of the Andreev bound states start to overlap which causes some features to disappear (Fig. 4.4).

At high bias, when all transitions are possible, the maximum current and the width of the current resonance are unaffected by $D$ or $M$. This is not immediately obvious from the transition rates due to the intricate forms of the prefactors. To investigate 


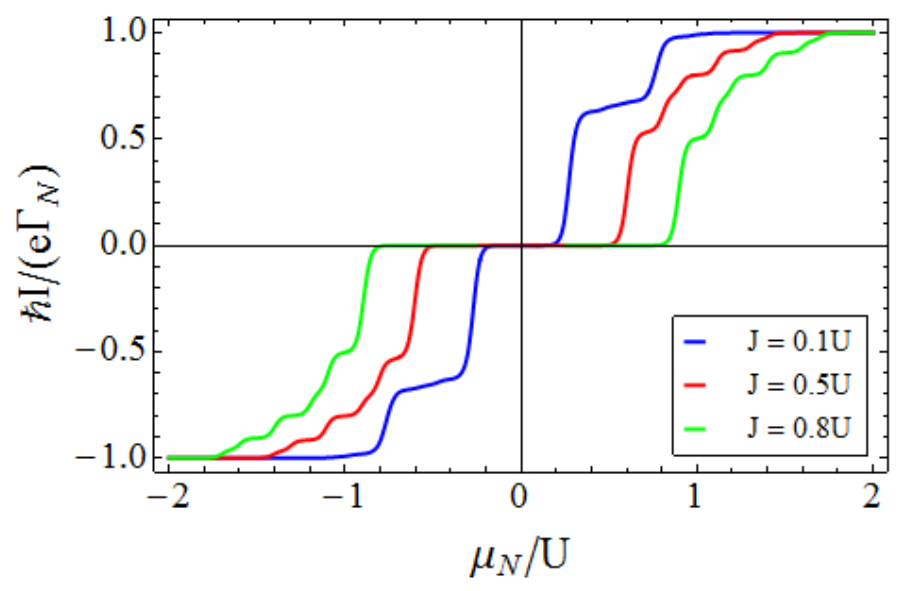

Figure 4.4: Plot of the current as a function of the chemical potential $\mu_{N}$ for $J=0.1$, 0.3 and $0.6 U$. The other parameters used in this plot are $\Gamma_{S}=0.5 U, \epsilon=-0.5 U$, $k_{B} T=0.02 U, D=0.2 U$ and $M=0.1 U$.

the effect of the $D$ and $M$ on the bias dependents of the current we have plotted the current as a function of $\mu_{N}$ in Figs. 4.5 and 4.6. For these plots $\epsilon=-0.5 U$, meaning the superconducting proximity effect is in resonance. Here we see that changing $D$ or $M$ does not have a significant effect on the current, especially compared to the effect of changing $J$ (Fig. 4.4). For the anisotropy this could be because, apart from the $|A\rangle$ states, all states are affected by the anisotropy to a similar degree and therefore the anisotropy mostly cancels out in the excitation energies.

As the changes in the current are relatively small as $D$ and $M$ are varied, we have plotted the differential conductance in Figs. 4.7 and 4.8, again for the superconducting proximity effect in resonance. In Fig. 4.7 the QTM is zero and the plots are made for various magnitudes of the anisotropy. In Fig. 4.8 the anistropy is zero and the plots are made for various QTM values. Here we see that for the smaller $D$ and $M$ values there are more differential conductance peaks. As $\left|\mu_{N}\right|$ is increased from zero the first peak is largest for greater $D$ and $M$ values. This is because when $D$ and $M$ are sufficiently large some of the excitation energies cross, meaning that when $\mu_{N}$ is high enough for the system to tunnel out of the ground state several transport channels are opened at once causing a greater increase in the current than for smaller $D$ and $M$, where the additional channels are opened at slightly higher $\left|\mu_{N}\right|$ values. For this system we have not plotted the excitation energies as there are too many for a visual representation to be meaningful, however the effect is the same described in reference to Fig. 3.10 in the previous chapter. In Figs. 4.9 and 4.10 we have plotted the differential conductance 


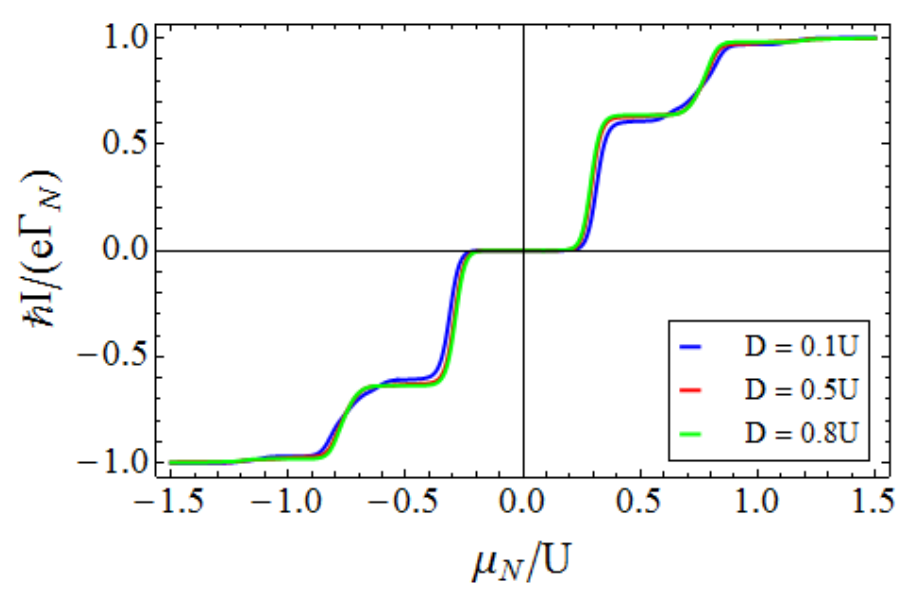

Figure 4.5: Plot of the current as a function of the chemical potential $\mu_{N}$ for $D=0.1$, 0.3 and $0.6 U$. The other parameters used in this plot are $\Gamma_{S}=0.5 U, \epsilon=-0.5 U$, $k_{B} T=0.02 U, J=0.2 U$ and $M=0.1 U$.

for $\epsilon=-1.1 U$. In these plots there is a negative differential conductance feature for $D=0.1 U$ and $M=0.1 U$, respectively. The feature for $D=0.1 U$ is however very small.

The expectation values of the electronic and molecular spins are zero for all parameter values. This can be deduced from the eigenstates. The electron spin expectation value is zero because the kets with opposite electron spins, in the $|C\rangle$ and $|D\rangle$ eigenstates, have the same prefactors and therefore contribute equally to the spin expectation value. For the same reasons the $|C\rangle$ and $|D\rangle$ states do not contribute to the expectation value of the molecular spin. The $|B\rangle$ states also do not give rise to non-zero molecular spin expectation values because for these states the magnitude of the prefactors of the kets with opposite molecular spins are the same.

\section{$4.4 \quad$ N-AMM-S Conclusions}

In this chapter we modified the model of Chapter 3 to allow for the molecule to be anisotropic. To see the affects of the anisotropy and QTM we chose the spin of the molecule to be $S=1$. The results for the current were similar to those found in the previous chapter. As a function of the applied bias and the level position, the current and differential conductance map out the excitation energies, meaning that current measurements should provide information on the anisotropy of a molecule. We found that the anisotropy and the QTM do not affect the maximum current or the resonance conditions of the proximity effect when the bias is greater than all excitation energies. 


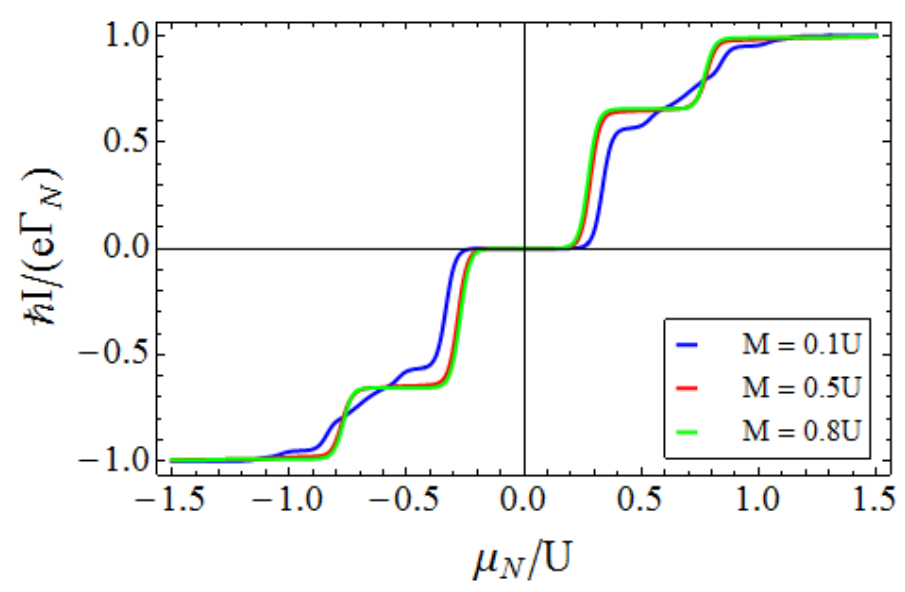

Figure 4.6: Plot of the current as a function of the chemical potential $\mu_{N}$ for $M=0.1$, 0.3 and $0.6 U$. The other parameters used in this plot are $\Gamma_{S}=0.5 U, \epsilon=-0.5 U$, $k_{B} T=0.02 U, J=0.2 U$ and $D=0.2 U$.

Although changes in the differential conductance can be observed as $D$ and $M$ are varied, these parameters have little effect on the current compared with the effect of the exchange coupling. Certain $D$ or $M$ values can however cause negative differential conductances. For this system we also find that the spin expectation values are zero, meaning that neither the spin of the electron or the molecule can be tuned using the bias voltage or by applying a gate voltage. 


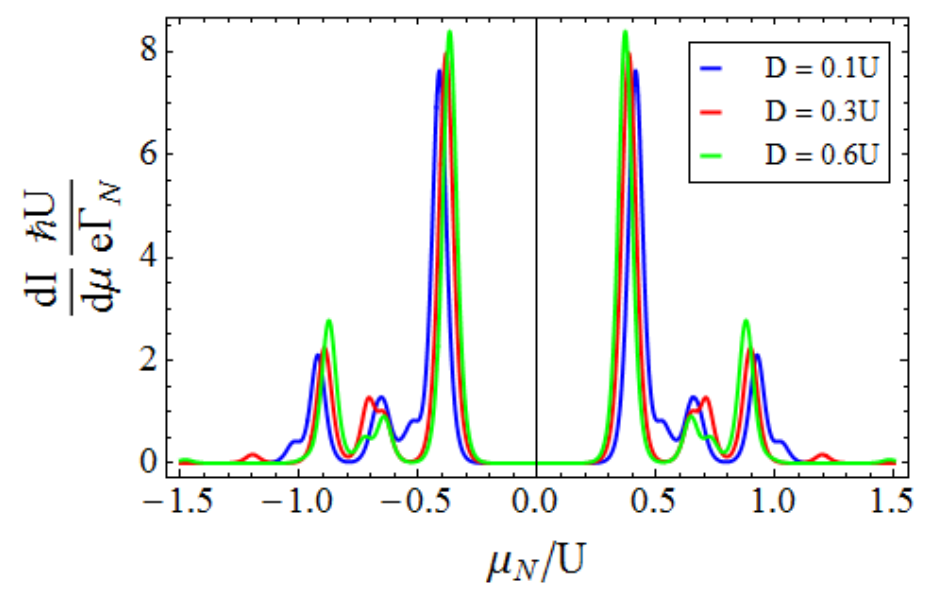

Figure 4.7: Plot of the differential conductance as a function of the chemical potential $\mu_{N}$ for $D=0.1,0.3$ and $0.6 U$. The other parameters used in this plot are $\Gamma_{S}=0.5 U$, $\epsilon=-0.5 U, k_{B} T=0.02 U, J=0.2 U$ and $M=0.1 U$.

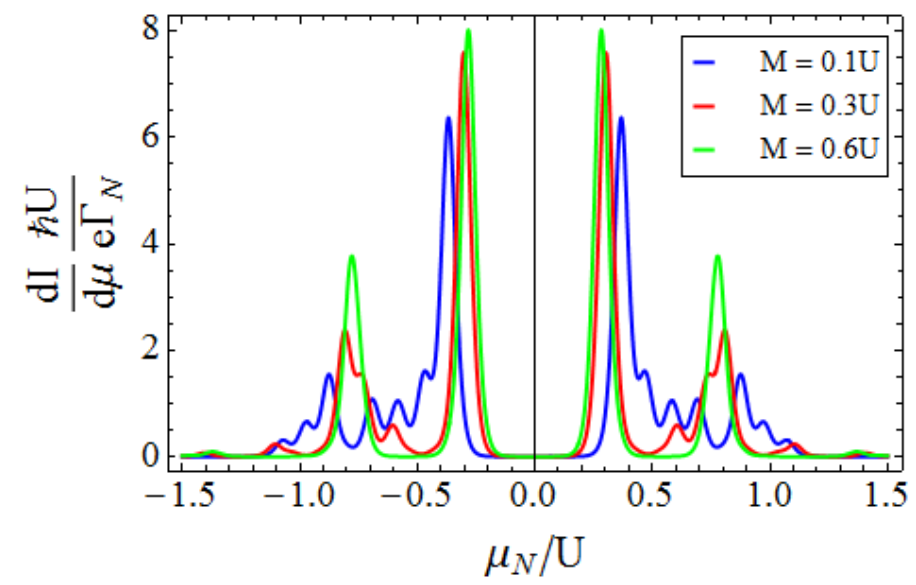

Figure 4.8: Plot of the differential conductance as a function of the chemical potential $\mu_{N}$ for $M=0.1,0.3$ and $0.6 U$. The other parameters used in this plot are $\Gamma_{S}=0.5 U$, $\epsilon=-0.5 U, k_{B} T=0.02 U, J=0.2 U$ and $D=0.2 U$. 


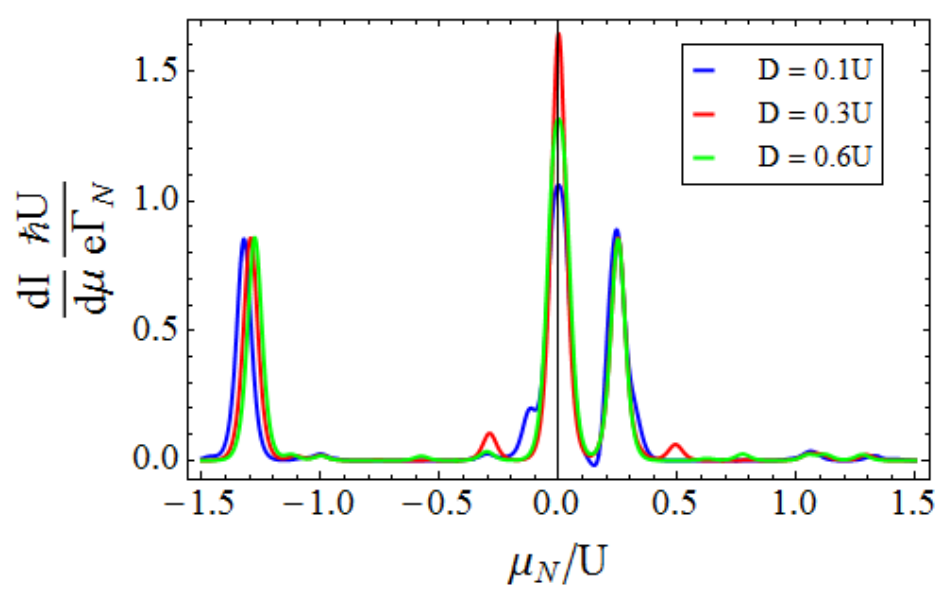

Figure 4.9: Plot of the differential conductance as a function of the chemical potential $\mu_{N}$ for $D=0.1,0.3$ and $0.6 U$. For $D=0.1 U$ there is negative differential conductance feature near $\mu_{N}=0.2 U$. The other parameters used in this plot are $\Gamma_{S}=0.5 U, \epsilon=-$ $1.1 U, k_{B} T=0.02 U, J=0.2 U$ and $M=0.1 U$.

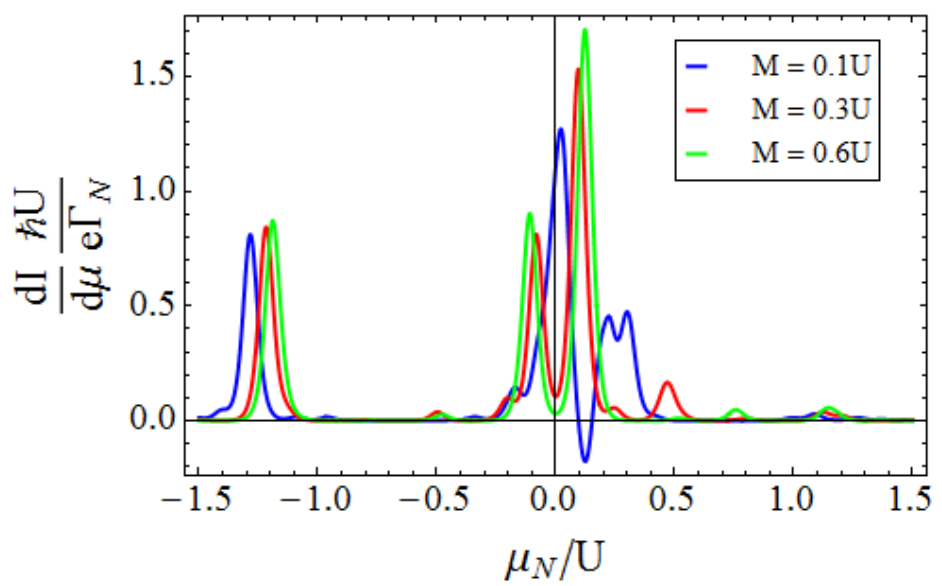

Figure 4.10: Plot of the differential conductance as a function of the chemical potential $\mu_{N}$ for $M=0.1,0.3$ and $0.6 U$. For $M=0.1 U$ there is negative differential conductance feature near $\mu_{N}=0.1 U$. The other parameters used in this plot are $\Gamma_{S}=0.5 U, \epsilon=-$ $1.1 U, k_{B} T=0.02 U, J=0.2 U$ and $D=0.2 U$. 


\section{Chapter 5}

\section{Josephson Current}

In the previous two chapters we have analysed the current through an isotropic and an anisotropic magnetic molecule coulped to normal and superconducting leads. In this chapter will replace the normal lead with a second superconducting lead to derive the Josephson current through the two magnetic molecules. Such systems have been analysed in recent years [20,21], however in these studies the superconducting gap was finite and the charging energy $U$ was infinite, and the current was only calculated to second order in tunnel coupling. In these limits the electrons of a Cooper pair cannot tunnel simultaneously to and from the superconducting leads. Instead, transport between the molecule and the leads involves the transfer of quasi-particles. We will once again work in the finite $U$ and $\Delta \rightarrow \infty$ limits and we will calculate the Josephson current to all orders in the coupling to the superconducting leads. In references [20] and $[21]$ it is found that for strong anti-ferromagnetic coupling between the electronic and molecular spins a $0-\pi$ transition is induced. For this transition to occur the sign of the current must change. This can be achieved if the order in which the electrons of a Cooper pair are created on the molecule is switched. However in the $\Delta \rightarrow \infty$ limit the electrons of a Cooper pair tunnel simultaneously, therefore no $0-\pi$ transition is excepted in this case.

\subsection{S-MM-S Systems}

To describe the molecules we use Eqs.3.2 and 4.1. The superconducting leads and tunneling coupling are described by

$$
H_{\eta}=\sum_{k, \sigma} \epsilon_{k} C_{\eta k \sigma}^{+} C_{\eta k \sigma}-\Delta \sum_{k}\left(C_{\eta-k \downarrow} C_{\eta k \uparrow}+H . c .\right)
$$




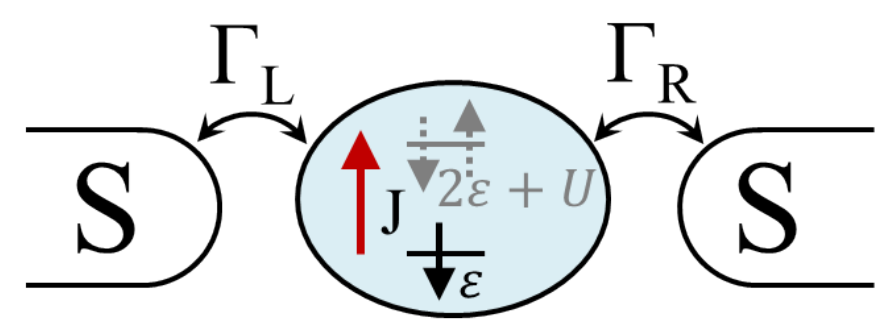

Figure 5.1: Single level magnetic molecule tunnel coupled to two superconducting leads, with coupling strengths $\Gamma_{L}, \Gamma_{R}$ and exchange coupling, $J$, between the spin of the electrons occupying the orbital level and the spin of the rest of the molecule.

and

$$
H_{t u n n, \eta}=\sum_{k, \sigma}\left(V_{\eta} C_{\eta k \sigma}^{+} d_{\sigma}+H . c .\right)
$$

where $\eta=L, R$ for the left and right superconducting leads. All other symbols have the same meaning as in Chapter 3.

We will calculate the Josephson current to all orders in tunnel coupling to the superconducting leads. In order to do this we once again use an effective Hamiltonian to describe the effect of coupling to these leads. For the case of a single level Anderson model dot between two superconducting leads the effective Hamiltonian was derived by Rozhkov and Arovas [50]. This Hamiltonian can easily be modified for the isotropic and anisotropic magnetic molecule cases by adding the exchange coupling, the anisotropy and the quantum tunneling of magnetisation terms. The effective Hamiltonians for the isotropic and anisotropic molecules are

$$
H_{I_{e f f}}=\sum_{\sigma} \epsilon d_{\sigma}^{+} d_{\sigma}+U n_{\uparrow} n_{\downarrow}+J \mathbf{S} . \mathbf{s}_{\mathbf{e}}-\frac{\chi_{S}}{2} d_{\uparrow}^{+} d_{\downarrow}^{+}-\frac{\chi_{S}^{*}}{2} d_{\downarrow} d_{\uparrow}
$$

and

$$
H_{A_{e f f}}=\sum_{\sigma} \epsilon d_{\sigma}^{+} d_{\sigma}+U n_{\uparrow} n_{\downarrow}-D S_{Z}^{2}+\frac{B_{2}}{2}\left(S_{+}^{2}+S_{-}^{2}\right)+J \mathbf{S} . \mathbf{s}_{\mathbf{e}}-\frac{\chi_{S}}{2} d_{\uparrow}^{+} d_{\downarrow}^{+}-\frac{\chi_{S}^{*}}{2} d_{\downarrow} d_{\uparrow},
$$

respectively. Here $\chi_{S}=\Gamma_{L} e^{i \phi_{L}}+\Gamma_{R} e^{i \phi_{R}}$, where $\Gamma_{\eta}$ and $\phi_{\eta}$ are the coupling strength and the phase of superconducting lead $\eta$. We once again choose $S=1 / 2$ for the isotropic case and $S=1$ for the anisotropic case.

The eigenstates of the effective Hamiltonians that involve single occupation of the 


\begin{tabular}{|c||c|}
\hline$E_{+ \pm}$ & $\frac{\delta}{2}+\frac{1}{2} \sqrt{\delta^{2}+\left|\chi_{S}\right|^{2}}$ \\
$E_{- \pm}$ & $\frac{\delta}{2}-\frac{1}{2} \sqrt{\delta^{2}+\left|\chi_{S}\right|^{2}}$ \\
$E_{T}$ & $\epsilon+\frac{J}{4}$ \\
$E_{S}$ & $\epsilon-\frac{3 J}{4}$ \\
\hline
\end{tabular}

Table 5.1: Energies of the 8 eigenstates of $H_{I, \text { eff }} . \delta$ is defined as in chapter 3 .

\begin{tabular}{|c||c|c|c|}
\hline$\chi$ & $A$ & $B$ & $C / D$ \\
\hline \hline$E_{\chi_{1}}$ & $\frac{\delta}{2}+\frac{1}{2} \sqrt{\delta^{2}+\left|\chi_{S}\right|^{2}}$ & $\frac{\delta}{2}-\frac{1}{2} \sqrt{\delta^{2}+\left|\chi_{S}\right|^{2}}-M-D$ & $\operatorname{Re}\left[\frac{\gamma+\beta^{2}+i \sqrt{3\left(\gamma-\beta^{2}\right)^{2}}}{12 \beta}\right]+\frac{3 \epsilon-2 D}{3}$ \\
$E_{\chi_{2}}$ & $\frac{\delta}{2}-\frac{1}{2} \sqrt{\delta^{2}+\left|\chi_{S}\right|^{2}}$ & $\frac{\delta}{2}-\frac{1}{2} \sqrt{\delta^{2}+\left|\chi_{S}\right|^{2}}+M-D$ & $\operatorname{Re}\left[\frac{\gamma+\beta^{2}-i \sqrt{3\left(\beta^{2}-\gamma\right)^{2}}}{12 \beta}\right]+\frac{3 \epsilon-2 D}{3}$ \\
$E_{\chi_{3}}$ & - & $\frac{\delta}{2}+\frac{1}{2} \sqrt{\delta^{2}+\left|\chi_{S}\right|^{2}}-M-D$ & $\operatorname{Re}\left[-\frac{\gamma+\beta^{2}}{6 \beta}\right]+\frac{3 \epsilon-2 D}{3}$ \\
$E_{\chi_{4}}$ & - & $\frac{\delta}{2}+\frac{1}{2} \sqrt{\delta^{2}+\left|\chi_{S}\right|^{2}}+M-D$ & - \\
\hline
\end{tabular}

Table 5.2: Energies of the 12 eigenstates of $H_{A, e f f} . \delta$ is defined as in chapter 3. The other terms are defined as $\beta=\left(\sqrt{\alpha}+72 M^{2} D-8 D^{3}+27 J^{3}\right)^{1 / 3}, \gamma=12 M^{2}+4 D^{2}+9 J^{2}$ and $\alpha=-\left(12 M^{2}+4 D^{2}+9 J^{2}\right)^{3}+\left(72 M^{2} D-8 D^{3}+27 J^{3}\right)^{2}$.

molecules are the same as those in Chapters 3 and 4 . This is because only the $|0\rangle_{e}$ and $|d\rangle_{e}$ states are affected by coupling to the extra superconducting lead. The remaining eigenstates are very similar to the bound states of the previous chapter; the only difference being that $\Gamma_{S}$ becomes $\chi_{S}$ or $\chi_{S}^{*}$. To calculate the Josephson current, the energies of the eigenstates are needed. These are given in Tables 5.1 and 5.2.

\subsection{Current}

The equilibrium Josephson current is calculated using Eq. 1.18 and the energies of the eigenstates of the two systems. We obtain analytic expressions. However, these are too long to be useful, thus in the next section we will investigate numerical results. 


\subsection{Results}

In the $J \rightarrow 0$ limit the there is no coupling between the electronic and molecular spins and the singly occupied states of the isotropic molecule become degenerate. In this limit the result for the Josephson current reduces to that for a single level quantum dot in place of the molecule. Governale et al. have calculated the Josephson current for the S-QD-S system, in the $\Delta \rightarrow \infty$ limit, to second order in the coupling to the leads. Setting $J=0, \Gamma_{L}=\Gamma_{R} \equiv \Gamma_{S}$ and taking the terms up to second order in the Taylor expansion of the current through the isotropic molecule, we find

$$
I_{\text {jos }}=\frac{\left(\mathrm{e}^{\frac{U+2 \epsilon}{k_{B} T}}-1\right) \Gamma_{S}^{2} \sin \phi}{\left(1+2 \mathrm{e}^{\frac{U+\epsilon}{k_{B} T}}+\mathrm{e}^{\frac{U+2 \epsilon}{k_{B} T}}\right)(U+2 \epsilon)}
$$

which is in agreement with the result of Governale et al. [5].
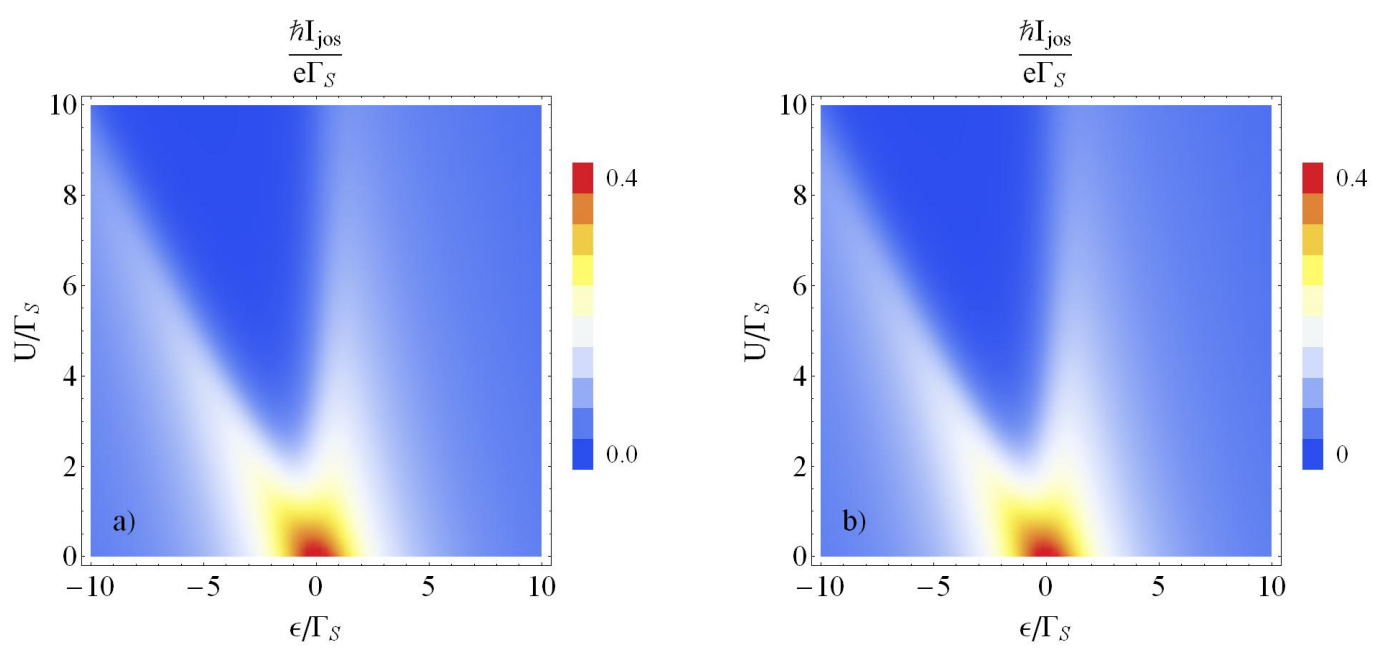

Figure 5.2: Density plots of the Josephson current through (a) the isotropic and (b) the anisotropic magnetic molecules as functions of the exchange coupling $U$ and the level position $\epsilon$. The other parameters used in these plots are $\phi=\pi / 2, k_{B} T=0.5 \Gamma_{S}$, $J=0.2 \Gamma_{S}, D=0.3 \Gamma_{S}$ and $M=0.2 \Gamma_{S}$.

Figures $5.2 \mathrm{a}$ ) and b) show density plots of the Josephson current for the isotropic and anisotropic molecules, respectively. Here and in all subsequent plots we have chosen $\Gamma_{L}=\Gamma_{R} \equiv \Gamma_{S}$. In plot b) there is no noticeable difference due to the anisotropy or the QTM compared with the isotropic molecule. Both plots show that as $U$ increases the current tends to zero in the negative $\epsilon$ half plane. In the positive $\epsilon$ half plane the ground states of the isotropic system are the $|- \pm\rangle$ states and these states contribute 
to the Josephson current. But for large $U>\epsilon$ in the negative $\epsilon$ half plane the ground state is $|S\rangle$, which does not contribute to the Josephson current, as this state does not couple to the superconducting leads. This occurs similarly for states of the anisotropic molecule. The current is greatest when $U=\epsilon=0$, because there the energy of the lowest bound state is minimised.

In Fig. 5.2 we see that the anisotropy and QTM of the anisotropic molecule do not have a significant effect on the current. To quantify the influence of these parameters we have plotted the current as functions of $D$ and $M$ in Figs. 5.3 and 5.4. In both cases the current increases with the magnitude of the parameter, but only by a few percent. This is probably because $D$ and $M$ affect all of the lowest energy eigenstates in a similar manner.

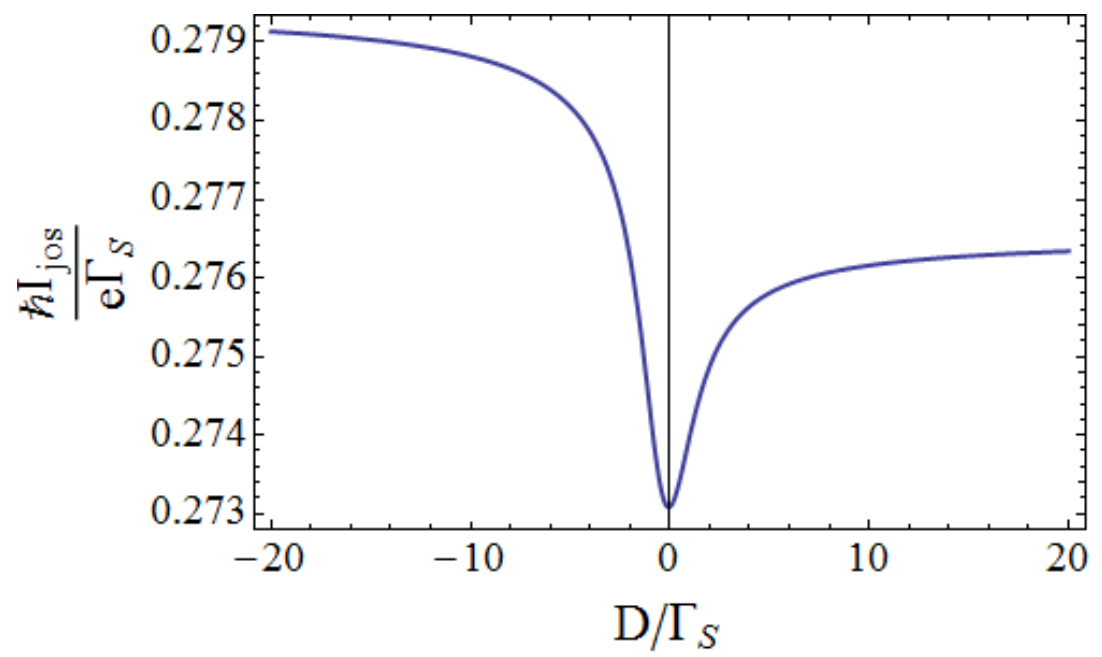

Figure 5.3: Plot of the current through the anisotropic molecule as a function of $D$ at $\delta=0$. The other parameters used in this plot are $\phi=\pi / 2, k_{B} T=0.5 \Gamma_{S}, J=0.2 \Gamma_{S}$ and $M=0.2 \Gamma_{S}$.

To analyse the affect of the exchange coupling we will concentrate on the isotropic molecule. Figure 5.5 a) and b) show density plots of the Josephson current for $J=3 \Gamma_{S}$ and $J=-3 \Gamma_{S}$. Compared with Fig. 5.2 a) the current is decreased for both plots. For large positive $J$ values the parameter range over which the singlet state is the ground state increases. This is shown by the larger zero current region. When $J$ is negative the triplet states are lower in energy than the singlet and are therefore the ground states in the zero current region.

Shown in Fig. 5.6 are density plots of the Josephson current through the isotropic molecule at $k_{B} T=0.1 \Gamma_{S}$ and $k_{B} T=1.0 \Gamma_{S}$. Note that the scales for these plots are 


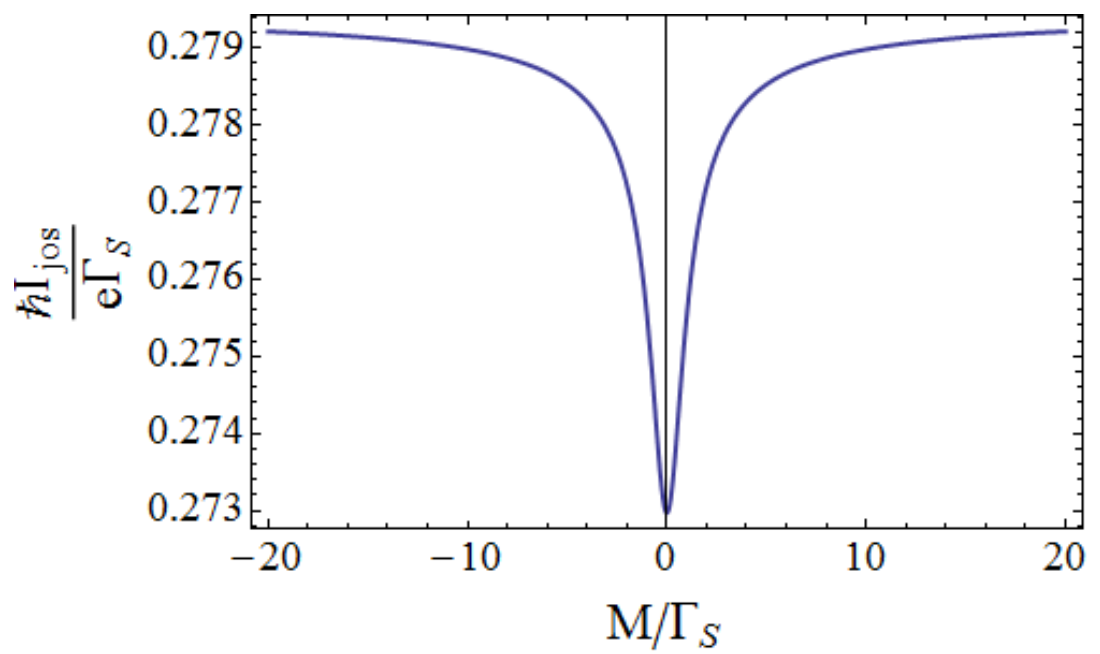

Figure 5.4: Plot of the current through the anisotropic molecule as a function of $M$ at $\delta=0$. The other parameters used in this plot are $\phi=\pi / 2, k_{B} T=0.5 \Gamma_{S}, J=0.2 \Gamma_{S}$ and $D=0.2 \Gamma_{S}$.

different. As expected, the transition form the zero current region to the non-zero current region becomes sharper as the temperature is lowered. In Fig. 5.2 a) $k_{B} T=0.5 \Gamma_{S}$. Comparing this plot with Fig. $5.6 \mathrm{~b}$ ) we see that with $U$ and $\epsilon$ scaled by the same factor as the temperature the current features appear the same, but the magnitude is approximately halved for the factor of two increase in the temperature. This trend occurs because as the temperature increases the eigenstates contribution more equally to the partition function, meaning that the denominator of Eq. 1.18 increases with respect to the numerator. We also see that in Fig. 5.6 a) the current is larger than for the higher temperature plots.

The phase dependence of the Josephson current is approximately sinusoidal. To second order the Josephson current through a quantum dot depends on $\sin \phi$ (Eq. 5.5), but because we have calculated the current to all orders in $\Gamma_{S}$ for the magnetic molecule systems, this is not exactly correct. However deviations from the sine dependence are not noticeable in the numerical results. We also find that for both the isotropic and the anisotropic cases there is no $0-\pi$ phase transition. As the parameters are varied the current can tend to zero, however it never changes sign. This is as expected since no quasi-particle states are allowed in the leads. 

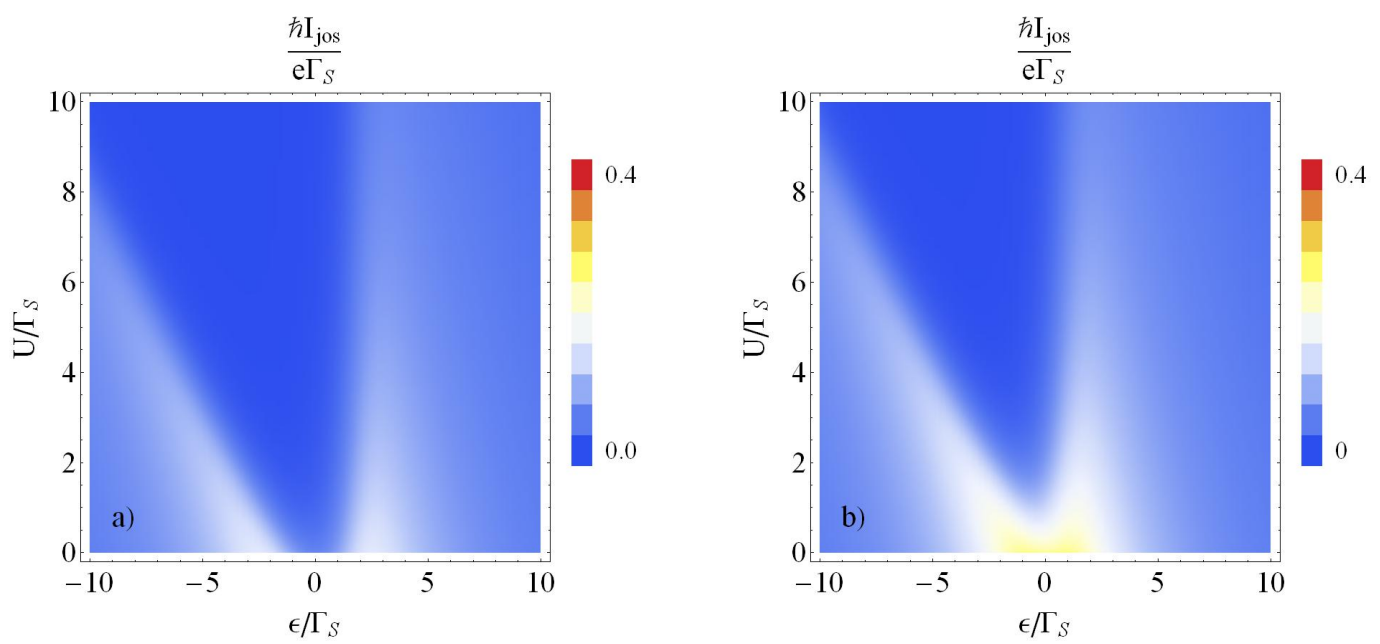

Figure 5.5: Density plots of the Josephson current through the isotropic magnetic molecule as a function of the exchange coupling $U$ and the level position $\epsilon$ for (a) $J=3 \Gamma_{S}$ and (b) $J=-3 \Gamma_{S}$. The other parameters used in these plots are $\phi=\pi / 2$ and $k_{B} T=0.5 \Gamma_{S}$.

\subsection{Josephson Current Conclusions}

In this chapter we calculated the equilibrium Josephson current through an isotropic and an anisotropic magnetic molecule coupled to two infinite gap superconducting leads. We found that no $0-\pi$ transition is induced for any parameter values and that the anisotropy and QTM have negligible affect on the current. Strong exchange coupling between the electronic and molecular spins decreases the current, as it lowers the energy of at least one of the singly occupied states and these do not contribute to the current. 

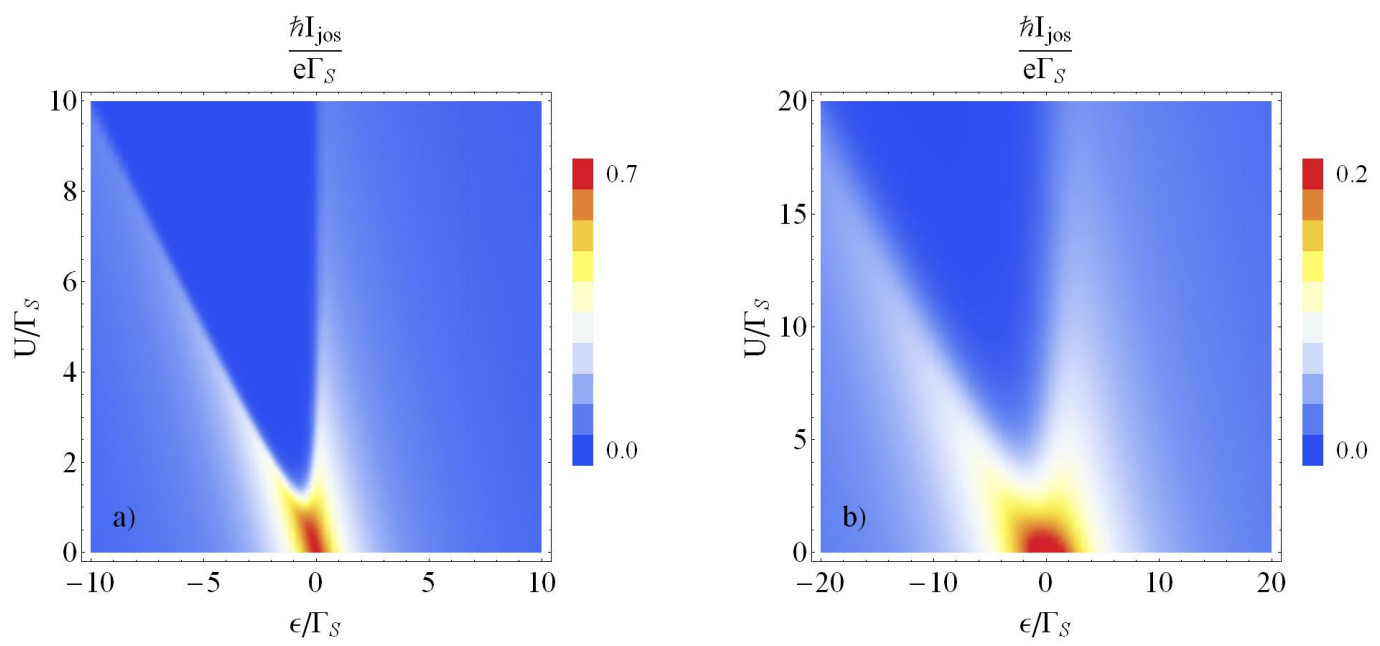

Figure 5.6: Density plots of the Josephson current through the isotropic as a function of the exchange coupling $U$ and the level position $\epsilon$ for (a) $k_{B} T=0.1 \Gamma_{S}$ and (b) $k_{B} T=1.0 \Gamma_{S}$. The other parameters used in these plots are $\phi=\pi / 2$ and $J=0.2 \Gamma_{S}$. 


\section{Chapter 6}

\section{Isotropic Magnetic Molecule Coupled to Ferromagnetic and BCS Leads}

In Chapter 3 we studied an isotropic magnetic molecule between a superconducting lead and a normal metallic lead. In this chapter we will modify this system to allow for the normal lead to be ferromagnetic and for the presence of an external magnetic field. The motivation for this is that it will add spin dependence to the current. In Chapter 3 we found that even though the molecule is magnetic the spin expectation values were zero, meaning that the current has no overall spin dependence. However with the normal lead now ferromagnetic more of the majority spins will tunnel to and from the molecule. This will change the non-equilibrium occupation probabilities of the eigenstates of the IMM-S subsystem and cause non-zero spin expectation values. For simplicity we will only consider the isotropic molecule in this chapter, as this shows interesting properties without having to work in the larger Hilbert space that is required for the anisotropic molecule.

We will allow for arbitrary alignment of the magnetisation of the ferromagnetic lead and the external magnetic field. This will lead to off-diagonal reduced density matrix elements contributing to the dynamics of the system. To calculate generalised transition rates involving these off-diagonal elements we will use the diagrammatic technique described in Chapter 2, since to the best our knowledge this is not possible using Fermi's golden rule. 


\subsection{F-IMM-S System}

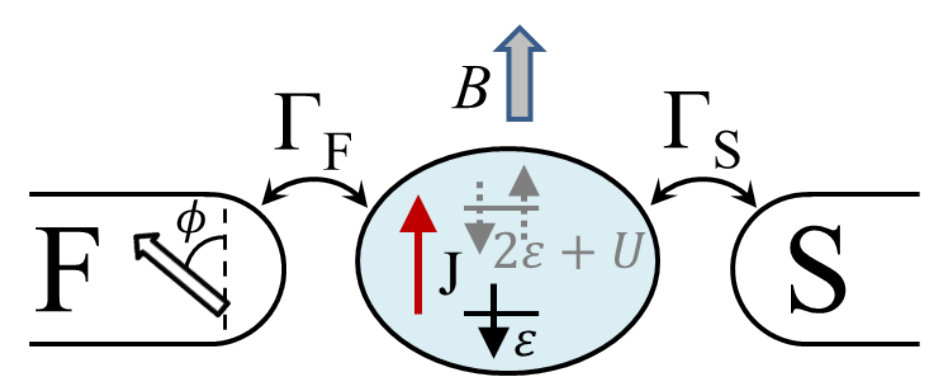

Figure 6.1: Single level isotropic magnetic molecule coupled to a superconducting lead and a ferromagnetic lead, in the presence of an external magnetic field, with coupling strengths $\Gamma_{S}$ and $\Gamma_{F}$, and exchange coupling, $J$, between the spin of the electrons occupying the orbital level and the spin of the rest of the molecule. The orientation of the external magnetic field is at an angle $\phi$ to the magnetisation of the ferromagnetic lead.

The modified system consists of a magnetic molecule, in the presence of an external magnetic field, tunnel coupled to a superconducting lead and ferromagnetic lead, as depicted in Fig. 6.1. The Hamiltonian of the system is given by

$$
H=H_{M}+H_{F}+H_{S}+H_{t u n n, F}+H_{t u n n, S}
$$

The Hamiltonian for the molecule in the presence of an external magnetic field is given by

$$
H_{M}=\sum_{\sigma} \epsilon d_{\sigma}^{+} d_{\sigma}+U n_{\uparrow} n_{\downarrow}+\frac{B_{1}}{2}\left(n_{\uparrow}-n_{\downarrow}\right)+B_{2} S_{z}+J \mathbf{S} . \mathbf{s}_{\mathbf{e}}
$$

where $\frac{B_{1}}{2}\left(n_{\uparrow}-n_{\downarrow}\right)$ describes the spin dependent Zeeman splitting of the orbital level due to the external magnetic field and $B_{2} S_{z}$ describes the effect the external magnetic field has on the spin of the molecule. All other terms have the same meaning as in Eq. 3.2.

Because we allow the magnetisation of the ferromagnetic lead to be arbitrarily aligned with respect to the orientation of the external magnetic field, the spin of the electrons occupying the lead will not be collinear with those occupying the molecule. We will therefore adopt a new notation to describe the orientation of the electrons occupying states in the ferromagnetic lead. The Hamiltonian for the ferromagnetic 
lead is now given by

$$
H_{F}=\sum_{k, \alpha} \epsilon_{k} C_{F, k, \alpha}^{+} C_{F, k, \alpha}
$$

where $\alpha=+,-$ stands for the majority and minority spins, respectively. The majority spins point in the direction of the magnetisation of the lead, whereas the minority spins point in the opposite direction. The Hamiltonian describing tunneling between the molecule and the ferromagnetic lead is given by

$$
H_{t u n n, F}=\sum_{k, \sigma, \alpha}\left(V_{F, \sigma, \alpha} C_{F, k, \alpha}^{+} d_{\sigma}+H . c .\right)
$$

The matrix elements $V_{F, \sigma, \alpha}$ are no longer diagonal in spin space due to the different quantisation axes of the lead and IMM-S subsystem. The individual tunneling amplitudes are given by $V_{F} U_{\sigma \alpha}$, where $U_{\sigma \alpha}$ is a $S U(2)$ rotation matrix, the form of which depends on the geometry of the system, and $V_{F}$ is the tunneling amplitude, which is assumed to be independent of spin and energy. We will consider the spin-quantisation axes of the lead and the IMM-S subsystem in the $x-z$ plane and use the matrix for rotations about the $y$-axis, $U_{y}(\phi)$. The tunneling Hamiltonian then becomes

$$
\begin{aligned}
H_{\text {tunn }, F}= & \sum_{k} V_{F}\left(\cos \left(\frac{\phi}{2}\right) C_{F k+}^{+} d_{\uparrow}-\sin \left(\frac{\phi}{2}\right) C_{F k+}^{+} d_{\downarrow}\right. \\
+ & \left.\sin \left(\frac{\phi}{2}\right) C_{F k-}^{+} d_{\uparrow}+\cos \left(\frac{\phi}{2}\right) C_{F k-}^{+} d_{\downarrow}+\text { H.c. }\right),
\end{aligned}
$$

where $\phi$ is the angle between the quantisation axes of the magnetisation of the ferromagnetic lead and the external magnetic field, as shown in Fig. 6.1. The maximum tunnel couplings for the majority and minority spins are given by $\Gamma_{F \pm}=2 \pi \rho_{ \pm}\left|V_{F}\right|^{2}$, where $\rho_{ \pm}$are the energy independent density of states for the majority and minority spins in the ferromagnetic lead. It is useful to express these tunnel coupling strengths in terms of $\Gamma_{F}=\frac{\Gamma_{F+}+\Gamma_{F-}}{2}$ and to use the polarisation $P=\frac{\rho_{+}-\rho_{-}}{\rho_{+}+\rho_{-}}$.

The Hamiltonians describing the superconducting lead and tunneling to and from the superconducting are still given by Eqs. 3.12 and 3.13 in Chapter 3, and once again we take into account the effect of the superconducting lead by introducing an effective Hamiltonian,

$$
H_{e f f}=H_{M}-\frac{\Gamma_{S}}{2}\left(d_{\uparrow}^{+} d_{\downarrow}^{+}+d_{\downarrow} d_{\uparrow}\right) .
$$

For simplicity we again choose the molecular spin to be $S=1 / 2$. Using the basis $\{|0,1 / 2\rangle,|d, 1 / 2\rangle,|0,-1 / 2\rangle,|d,-1 / 2\rangle,|\uparrow, 1 / 2\rangle,|\downarrow,-1 / 2\rangle,|\downarrow, 1 / 2\rangle,|\uparrow,-1 / 2\rangle\}$, we can write the effective Hamiltonian in matrix form. This matrix is block diagonal and can 
therefore be broken into two blocks, the first involving the empty and doubly occupied states and the second involving the singly occupied states:

$$
\begin{gathered}
H_{e f f 1}=\left(\begin{array}{cccc}
\frac{B_{2}}{2} & \frac{-\Gamma_{S}}{2} & 0 & 0 \\
\frac{-\Gamma_{S}}{2} & 2 \epsilon+U+\frac{B_{2}}{2} & 0 & 0 \\
0 & 0 & \frac{-B_{2}}{2} & \frac{-\Gamma_{S}}{2} \\
0 & 0 & \frac{-\Gamma_{S}}{2} & 2 \epsilon+U-\frac{B_{2}}{2}
\end{array}\right) \\
H_{e f f 2}=\left(\begin{array}{cccc}
\epsilon+\frac{J}{4}+\frac{B_{1}+B_{2}}{2} & 0 & 0 & 0 \\
0 & \epsilon+\frac{J}{4}-\frac{B_{1}+B_{2}}{2} & 0 & 0 \\
0 & 0 & \epsilon-\frac{J}{4}-\frac{B_{1}-B_{2}}{2} & \frac{J}{2} \\
0 & 0 & \frac{J}{2} & \epsilon-\frac{J}{4}+\frac{B_{1}-B_{2}}{2}
\end{array}\right) .
\end{gathered}
$$

We diagonalise these matrices to find the energies and eigenstates of this system for zero coupling to the ferromagnetic lead. Compared with the eigenstates of the system studied in Chapter 3 the degeneracy of the Andreev bound state pairs has been lifted due to the magnetic field acting on the molecular spin. The four bound states and their respective energies are now given by

$$
\begin{gathered}
|+, \pm\rangle=\frac{1}{\sqrt{2}} \sqrt{1-\frac{\delta}{2 \epsilon_{A}}}|0, \pm 1 / 2\rangle-\frac{1}{\sqrt{2}} \sqrt{1+\frac{\delta}{2 \epsilon_{A}}}|d, \pm 1 / 2\rangle \\
E_{+ \pm}=\frac{\delta}{2}+\epsilon_{A} \pm \frac{B_{2}}{2}
\end{gathered}
$$

and

$$
\begin{gathered}
|-, \pm\rangle=\frac{1}{\sqrt{2}} \sqrt{1+\frac{\delta}{2 \epsilon_{A}}}|0, \pm 1 / 2\rangle+\frac{1}{\sqrt{2}} \sqrt{1-\frac{\delta}{2 \epsilon_{A}}}|d, \pm 1 / 2\rangle \\
E_{- \pm}=\frac{\delta}{2}-\epsilon_{A} \pm \frac{B_{2}}{2}
\end{gathered}
$$

The definitions of $\delta$ and $\epsilon_{A}$ are those given in Chapter 3.

In Chapter 3 the remaining four states formed a singlet and a triplet. The states in this system still resemble those of Chapter 3 but the degeneracy of the triplet states has been lifted. The states and their energies are now given by

$$
\begin{gathered}
|I+\rangle=|\uparrow, 1 / 2\rangle \\
E_{I+}=\epsilon+\frac{J}{4}+\frac{B_{1}+B_{2}}{2},
\end{gathered}
$$




$$
\begin{gathered}
|I-\rangle=|\downarrow,-1 / 2\rangle \\
E_{I-}=\epsilon+\frac{J}{4}-\frac{B_{1}+B_{2}}{2}, \\
|I 0\rangle=\frac{\lambda_{1+}}{\sqrt{2}}|\downarrow, 1 / 2\rangle+\frac{\lambda_{2+}}{\sqrt{2}}|\uparrow,-1 / 2\rangle \\
E_{I 0}=\epsilon-\frac{J}{4}+\frac{1}{2} \sqrt{\left(B_{1}-B_{2}\right)^{2}+J^{2}}
\end{gathered}
$$

and

$$
\begin{aligned}
& |I S\rangle=\frac{\lambda_{1-}}{\sqrt{2}}|\downarrow, 1 / 2\rangle-\frac{\lambda_{2-}}{\sqrt{2}}|\uparrow,-1 / 2\rangle \\
& E_{I S}=\epsilon-\frac{J}{4}-\frac{1}{2} \sqrt{\left(B_{1}-B_{2}\right)^{2}+J^{2}} .
\end{aligned}
$$

The prefactors of the kets in the states $|I S\rangle$ and $|I 0\rangle$ are

$$
\lambda_{1 \pm}=\sqrt{\frac{J^{2}}{J^{2}+\left(B_{1}-B_{2}\right)^{2} \pm\left(B_{1}-B_{2}\right) \sqrt{\left(B_{1}-B_{2}\right)^{2}+J^{2}}}}
$$

and

$$
\lambda_{2 \pm}=\sqrt{\frac{J^{2}+2\left(B_{1}-B_{2}\right)^{2} \pm 2\left(B_{1}-B_{2}\right) \sqrt{\left(B_{1}-B_{2}\right)^{2}+J^{2}}}{J^{2}+\left(B_{1}-B_{2}\right)^{2} \pm\left(B_{1}-B_{2}\right) \sqrt{\left(B_{1}-B_{2}\right)^{2}+J^{2}}}} .
$$

In the limit $B_{1}=B_{2} \rightarrow 0$ the eigenstates reduce to the Andreev bound states and triplet-singlet of Chapter 3. We will label the excitation energies as $\left|E_{a b}\right|= \pm\left|E_{a}-E_{b}\right|$.

\subsection{Transition Rates and Current}

When $\phi \neq 0$ the spin of the electron is not conserved in individual tunneling events between the molecule and the ferromagnetic lead. Due to this there are off-diagonal reduced density matrix elements that can contribute to the dynamics of the system. However, when $\phi=0$ no off-diagonal elements should be included. We will therefore treat the two cases separately. First we will calculate the rates for the collinear case, $\phi=0$, then we will consider the non-collinear case, $\phi \neq 0$. 


\subsubsection{Collinear}

As the electronic and molecular spins are conserved during tunneling events the generalised transition rates contributing to the dynamics of the system are still restricted to those given in Chapter 3. We do not have to consider any off-diagonal reduced density matrix elements and therefore can once again use Fermi's golden rule to calculated the first order rates. Because in this case the spin of the electrons in the ferromagnetic lead are aligned with those on the molecule it is more convenient to let $\alpha=\uparrow, \downarrow$ instead of,+- . Therefore we define the polarisation and average coupling strength as

$$
P=\frac{\rho_{\uparrow}-\rho_{\downarrow}}{\rho_{\uparrow}+\rho_{\downarrow}}
$$

and

$$
\Gamma_{F}=\frac{\Gamma_{F \uparrow}+\Gamma_{F \downarrow}}{2}
$$

respectively. It is convenient to write $\Gamma_{F \uparrow}$ and $\Gamma_{F \downarrow}$ in terms of $P$ and $\Gamma_{F}$, giving

$$
\Gamma_{F \uparrow}=(1+P) \Gamma_{F}
$$

and

$$
\Gamma_{F \downarrow}=(1-P) \Gamma_{F}
$$

The non-zero transition rates are

$$
\begin{aligned}
W_{ \pm+I+}= & \frac{\Gamma_{F}}{2}\left[(1-P) \delta_{ \pm}^{2} f^{+}\left(E_{ \pm+}-E_{I+}\right)+(1+P) \delta_{\mp}^{2} f^{-}\left(E_{I+}-E_{ \pm+}\right)\right] \\
W_{I+ \pm+}= & \frac{\Gamma_{F}}{2}\left[(1-P) \delta_{ \pm}^{2} f^{-}\left(E_{ \pm+}-E_{I+}\right)+(1+P) \delta_{\mp}^{2} f^{+}\left(E_{I+}-E_{ \pm+}\right)\right] \\
W_{ \pm-I-}= & \frac{\Gamma_{F}}{2}\left[(1+P) \delta_{ \pm}^{2} f^{+}\left(E_{ \pm-}-E_{I-}\right)+(1-P) \delta_{\mp}^{2} f^{-}\left(E_{I-}-E_{ \pm-}\right)\right] \\
W_{I- \pm-}= & \frac{\Gamma_{F}}{2}\left[(1+P) \delta_{ \pm}^{2} f^{-}\left(E_{ \pm-}-E_{I-}\right)+(1-P) \delta_{\mp}^{2} f^{+}\left(E_{I-}-E_{ \pm-}\right)\right] \\
W_{ \pm+\eta}= & \frac{\Gamma_{F} \lambda_{1 \zeta}^{2}}{4}\left[(1+P) \delta_{ \pm}^{2} f^{+}\left(E_{ \pm+}-E_{\eta}\right)+(1-P) \delta_{\mp}^{2} f^{-}\left(E_{\eta}-E_{ \pm+}\right)\right] \\
W_{\eta \pm+}= & \frac{\Gamma_{F} \lambda_{1 \zeta}^{2}}{4}\left[(1+P) \delta_{ \pm}^{2} f^{-}\left(E_{ \pm+}-E_{\eta}\right)+(1-P) \delta_{\mp}^{2} f^{+}\left(E_{\eta}-E_{ \pm+}\right)\right] \\
W_{ \pm-\eta}= & \frac{\Gamma_{F} \lambda_{2 \zeta}^{2}}{4}\left[(1-P) \delta_{ \pm}^{2} f^{+}\left(E_{ \pm-}-E_{\eta}\right)+(1+P) \delta_{\mp}^{2} f^{-}\left(E_{\eta}-E_{ \pm-}\right)\right]
\end{aligned}
$$


and

$$
W_{\eta \pm-}=\frac{\Gamma_{F} \lambda_{2 \zeta}^{2}}{4}\left[(1-P) \delta_{ \pm}^{2} f^{-}\left(E_{ \pm-}-E_{\eta}\right)+(1+P) \delta_{\mp}^{2} f^{+}\left(E_{\eta}-E_{ \pm-}\right)\right] .
$$

Here $\eta=I 0, I S$ and $\zeta=+$ if $\eta=I 0$ or $\zeta=-$ if $\eta=I S$. As in Chapter $3 \delta_{ \pm}^{2}=\left(1 \pm \frac{\delta}{2 \epsilon_{A}}\right)$.

The occupation probabilities, current and Fano factor are calculated using the same methods as in Chapter 3.

\subsubsection{Non-collinear}

With eight eigenstates and without the requirement that the electronic spin be conserved during tunneling events there are now off-diagonal reduced density matrix elements that effect the dynamics of the system. In the most general case there are over 200 generalised transition rates that are non-zero. Table 6.1 summarises the coherent superpositions that must be taken into account for various parameter values. For simplicity we have set $B_{1}=B_{2}=B$. To make the system of equations more manageable we will consider only case (iv)-b. The condition $\left|2 \epsilon_{A}-B\right| \lesssim \Gamma_{N}$ is satisfied when $2 \epsilon_{A}-B=0$. This can be rewritten to give the condition $\delta= \pm \sqrt{B^{2}-\Gamma_{S}^{2}}$. In this regime there are only two off-diagonal reduced density matrix elements that contribute to the current. The reduced density matrix of the molecule coupled to the superconducting lead, in this regime, is

$$
\rho_{M}^{R}=\left(\begin{array}{cccccccc}
P_{++} & 0 & 0 & 0 & P_{T 1}^{++} & 0 & 0 & 0 \\
0 & P_{+-} & P_{-+}^{+-} & 0 & 0 & 0 & P_{T 0}^{+-} & P_{S}^{+-} \\
0 & P_{+-}^{-+} & P_{-+} & 0 & 0 & 0 & P_{T 0}^{-+} & P_{S}^{-+} \\
0 & 0 & 0 & P_{--} & 0 & P_{T-1}^{--} & 0 & 0 \\
P_{++}^{T 1} & 0 & 0 & 0 & P_{T 1} & 0 & 0 & 0 \\
0 & 0 & 0 & P_{--}^{T-1} & 0 & P_{T-1} & 0 & 0 \\
0 & P_{+-}^{T 0} & P_{-+}^{T 0} & 0 & 0 & 0 & P_{T 0} & 0 \\
0 & P_{+-}^{S} & P_{-+}^{S} & 0 & 0 & 0 & 0 & P_{S}
\end{array}\right) .
$$

The entries in red are non-zero but in first order they cannot couple to the diagonal elements and therefore do not contribute to the dynamics of the system. With the ten reduced density matrix elements that contribute to the dynamics of the system there are 42 non-zero generalised transition rates that must be calculated.

As there are now non-zero off-diagonal reduced density matrix elements that contribute to the dynamics of the system we can no longer use Fermi's golden rule to 


\begin{tabular}{|c|c|c|}
\hline Case & Parameters & Superpositions \\
\hline \hline (i) & $B \lesssim \Gamma_{N}, J \gg \Gamma_{N}$ & $\begin{array}{c}|I+\rangle,|I-\rangle,|I 0\rangle \\
| \pm \pm\rangle,| \pm \mp\rangle\end{array}$ \\
\hline (ii) & $B, J \lesssim \Gamma_{N}$ & $|I+\rangle,|I-\rangle,|I 0\rangle$ \\
& & $|I \pm\rangle,|I S\rangle$ \\
& & $|I 0\rangle,|I S\rangle$ \\
& & $| \pm \pm\rangle,| \pm \mp\rangle$ \\
\hline (iii) & $J \lesssim \Gamma_{N}, B \gg \Gamma_{N}$ & $|I 0\rangle,|I S\rangle$ \\
a & $\left(\right.$ if $\left.\left|2 \epsilon_{A}-B\right| \lesssim \Gamma_{N}\right)$ & $(|+-\rangle,|-+\rangle)$ \\
\hline (iv) & $B, J \gg \Gamma_{N}$ & none \\
a & $\left(\right.$ if $\left.|J-B| \lesssim \Gamma_{N}\right)$ & $(|I-\rangle,|I S\rangle)$ \\
b & $\left(\right.$ if $\left.\left|2 \epsilon_{A}-B\right| \lesssim \Gamma_{N}\right)$ & $(|+-\rangle,|-+\rangle)$ \\
\hline
\end{tabular}

Table 6.1: Coherent superpositions of that have to be taken into account for various parameter values are given in this table.

calculate all of the rates. Therefore we will use the diagrammatic technique outlined in Chapter 2. Each generalised transition rate, $W_{\chi_{1} \chi_{1}^{\prime}}^{\chi_{2}}$, can be represented by diagrams such as that shown in Fig. 6.2. Then a set of rules can be used to write out the equations represented by these diagrams. To calculate the generalised transition rates of this system, to first order in tunnel coupling $\Gamma_{F}$, the diagrammatic rules are:

1. Draw all topologically different diagrams and assign energies to all propagators and tunneling lines.

2. For each part of the diagram between adjacent vertices assign a resolvent $\frac{1}{\Delta E+i 0^{+}}$, where $\Delta E$ is the energy difference between left going and right going propagators and tunneling lines.

3. For each tunneling line the diagram acquires a factor of $f^{ \pm}(\omega)$, - $(+)$ for lines running forward (backward) with respect to the Keldysh contour.

4. For each pair of vertices connected by a tunneling line the diagram is multiplied by

$$
\rho_{+}\left\langle\chi_{i}^{\prime}\left|C_{+\sigma}\right| \chi_{i}\right\rangle\left\langle\chi_{f}^{\prime}\left|C_{+\sigma}^{+}\right| \chi_{f}\right\rangle+\rho_{-}\left\langle\chi_{i}^{\prime}\left|C_{-\sigma}\right| \chi_{i}\right\rangle\left\langle\chi_{f}^{\prime}\left|C_{-\sigma}^{+}\right| \chi_{f}\right\rangle
$$

where $\chi_{i}$ and $\chi_{i}^{\prime}\left(\chi_{f}\right.$ and $\left.\chi_{f}^{\prime}\right)$ are the states that enter and leave the vertex where the tunneling line begins (ends), respectively. The operators $C_{\alpha \sigma}^{(+)}$are the 
coefficients (including the dot operators) of $C_{F k \alpha}^{(+)}$in the tunneling Hamiltonian.

5. The diagram is multiplied by a factor of $-i(-1)^{a}$, where a is the number of vertices on the lower propagator.

6. Integrate the diagram over all energies $\omega$.

7. When calculating the generalised current rates multiply each diagram by 1 if the line is going form the lower to the upper propagator and -1 if it is going from the upper to the lower propagator, otherwise multiply the diagram by zero.

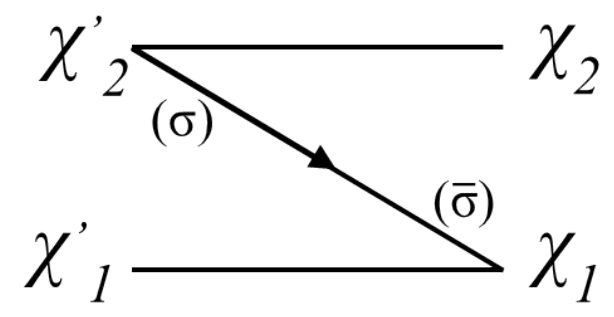

Figure 6.2: One of the possible transitions from $P_{\chi_{1}}^{\chi_{2}}$ to $P_{\chi_{1}^{\prime}}^{\chi_{2}^{\prime}}$. The top line represents forward propagation in time and the bottom line backward propagation. The line with the arrow head is the tunneling line and represents the contraction of two tunneling vertices. The tunneling line points to where an electron is created on the orbital level of the molecule. The spin of the electrons destroyed and created on the dot are represented by $\sigma$ and $\bar{\sigma}$; these can be the same or different.

Figure 6.3 shows the diagrammatic representation of $W_{-+-+}^{+-+-}$. Applying the diagrammatic rules to Fig. 6.3, and rewriting the equation in terms of $\Gamma_{F}$ and $P$, we obtain

$$
\begin{gathered}
W_{-+-+}^{+-+-}=\frac{-i}{4 \pi}\left\{\Gamma _ { F } ( 1 + P \operatorname { c o s } \phi ) \left(\delta_{+}^{2}\left[\int \frac{f^{-}(\omega) d \omega}{E_{-+}-E_{I-}-\omega+i 0^{+}}+\int \frac{f^{+}(\omega) d \omega}{E_{I+}-E_{+-}-\omega+i 0^{+}}\right]+\right.\right. \\
\left.\frac{\delta_{-}^{2}}{2}\left[\int \frac{f^{+}(\omega) d \omega}{E_{-+}-E_{I 0}+\omega+i 0^{+}}+\int \frac{f^{+}(\omega) d \omega}{E_{-+}-E_{I S}+\omega+i 0^{+}}+\int \frac{f^{-}(\omega) d \omega}{E_{I 0}-E_{+-}+\omega+i 0^{+}}+\int \frac{f^{-}(\omega) d \omega}{E_{I S}-E_{+-}+\omega+i 0^{+}}\right]\right) \\
\quad+\Gamma_{F}(1-P \cos \phi)\left(\delta_{-}^{2}\left[\int \frac{f^{+}(\omega) d \omega}{E_{-+}-E_{I-}+\omega+i 0^{+}}+\int \frac{f^{-}(\omega) d \omega}{E_{I+}-E_{+-}+\omega+i 0^{+}}\right]+\right. \\
\left.\left.\frac{\delta_{+}^{2}}{2}\left[\int \frac{f^{-}(\omega) d \omega}{E_{-+}-E_{I 0}-\omega+i 0^{+}}+\int \frac{f^{-}(\omega) d \omega}{E_{-+}-E_{I S}-\omega+i 0^{+}}+\int \frac{f^{+}(\omega) d \omega}{E_{I 0^{-}-E_{+-}-\omega+i 0^{+}}}+\int \frac{f^{+}(\omega) d \omega}{E_{I S^{-}-E_{+-}-\omega+i 0^{+}}}\right]\right)\right\} .
\end{gathered}
$$

These integrals are then calculated using the residue theorem, which is given in Appendix $\mathrm{C}$ along with an example calculation. The expression for $W_{-+-+}^{+-+}$is rather 


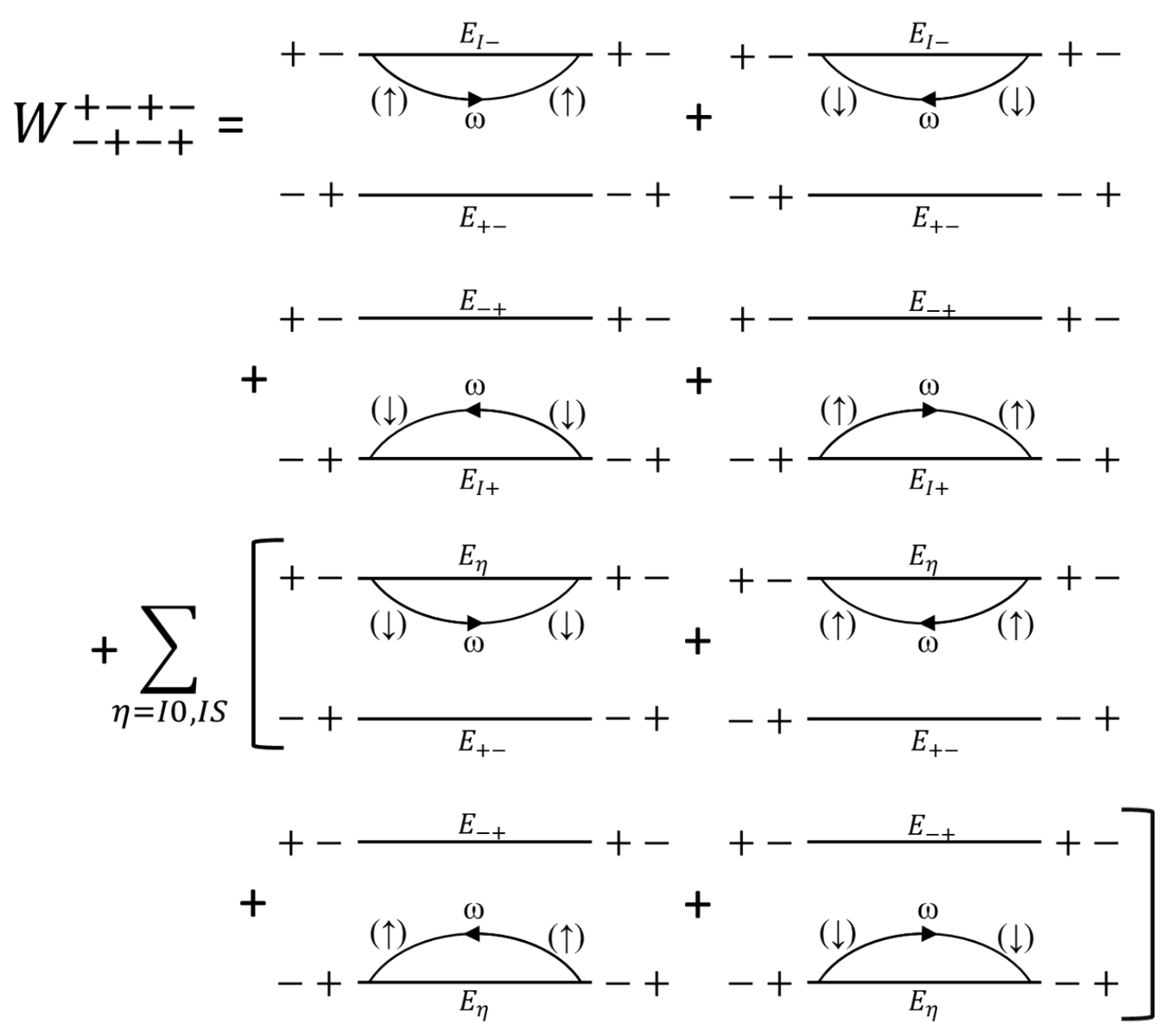

Figure 6.3: All of the first order contributions to the generalised transition rate $W_{-+-+}^{+--}$.

cumbersome and is therefore given in Appendix C, with all other non-zero generalised transition rates. Here we give two example rates;

$$
W_{I+ \pm+}=\frac{\Gamma_{F}}{2}\left[\delta_{ \pm}^{2}(1-P \cos \phi) f^{-}\left(E_{ \pm+}-E_{I+}\right)+\delta_{\mp}^{2}(1+P \cos \phi) f^{+}\left(E_{I+}-E_{ \pm+}\right)\right]
$$

and

$$
\begin{gathered}
W_{\mp \pm \eta}^{ \pm \mp \eta}=\alpha \frac{i \delta_{+} \delta_{-}}{8 \pi} \Gamma_{F} P \sin \phi\left(i \pi \left(f^{+}\left(E_{+-}-E_{\eta}\right)+f^{+}\left(E_{-+}-E_{\eta}\right)+f^{-}\left(E_{\eta}-E_{-+}\right)+\right.\right. \\
\left.f^{-}\left(E_{\eta}-E_{+-}\right)\right) \mp \operatorname{Re}\left[\Psi\left(\frac{1}{2}+\frac{i \beta}{2 \pi}\left(E_{\eta}-E_{+-}-\mu_{F}\right)\right)-\Psi\left(\frac{1}{2}+\frac{i \beta}{2 \pi}\left(E_{\eta}-E_{-+}-\mu_{F}\right)\right)\right] \\
\left.\mp \operatorname{Re}\left[\Psi\left(\frac{1}{2}+\frac{i \beta}{2 \pi}\left(E_{+-}-E_{\eta}-\mu_{F}\right)\right)-\Psi\left(\frac{1}{2}+\frac{i \beta}{2 \pi}\left(E_{-+}-E_{\eta}-\mu_{F}\right)\right)\right]\right) .
\end{gathered}
$$

$\Psi(x)$ is the digamma function, $\eta=I 0, I S$ and $\alpha=+(-)$ if $\eta=I 0(I S)$.

To calculate the current we modify the generalised transition rates to obtain the 
current rates. This is done by applying rule 7 , given in the list of diagrammatic rule presented in this section. These current rates are then used in Eq. 2.24. For the noncollinear case we will not calculate the Fano factor as the method described in Chapter 2 is only valid for systems where no off-diagonal density matrix elements contribute to the current.

\subsection{Results - Collinear}

In this section we will investigate the results of the collinear case. As in Chapter 3 it was not possible to obtain analytic expressions for the occupation probabilities or the current. We therefore present numerical results in graphical form in this section. Firstly we consider the case where the external field is switched off, in which case the energies and eigenstates reduce to those in Chapter 3. Secondly we consider the case where the molecule is in the presence of an external magnetic field, but switch off the magnetisation of the ferromagnetic lead. Lastly we look at the most general case where both the magnetisation of the metallic lead and the external magnetic field are non zero.

\subsubsection{Ferromagnetic Lead, $\mathrm{B}=0$}

The magnetisation of the metallic lead is introduced into the model via spin dependent density of states. Having a finite polarisation will reduce the likelihood of tunneling events involving the minority spin. If the polarisation is positive and we consider the case where the molecule is singly occupied with a spin up electron, and the applied bias favours transport from the ferromagnetic lead to the molecule, then the effect of this polarisation is to increase the waiting time for the transfer of a spin down electron. This means that the maximum current in the system is reduced compared with the non-magnetic lead case. Figure 6.4 shows density plots of the current for polarisations of 0.4 and 0.9. Note that the colour scales for the two plots are different. Here we see that the maximum current, which is achieved at high bias and zero detuning, is reduced with increased polarisation. For $P=0.4$ the maximum current is reduced by about 10 percent compared with an unpolarised lead and for $P=0.9$ by about 80 percent. The magnitude of the maximum current is given by $\left|I_{\max }\right|=\Gamma_{F}\left(1-P^{2}\right)$. As expected, when $P= \pm 1$ no current flows through the system. This is because in this case there is only one type of spin in the ferromagnetic lead and no possibilities of spin flips.

The polarisation of the lead also changes the relative magnitude of the current for 

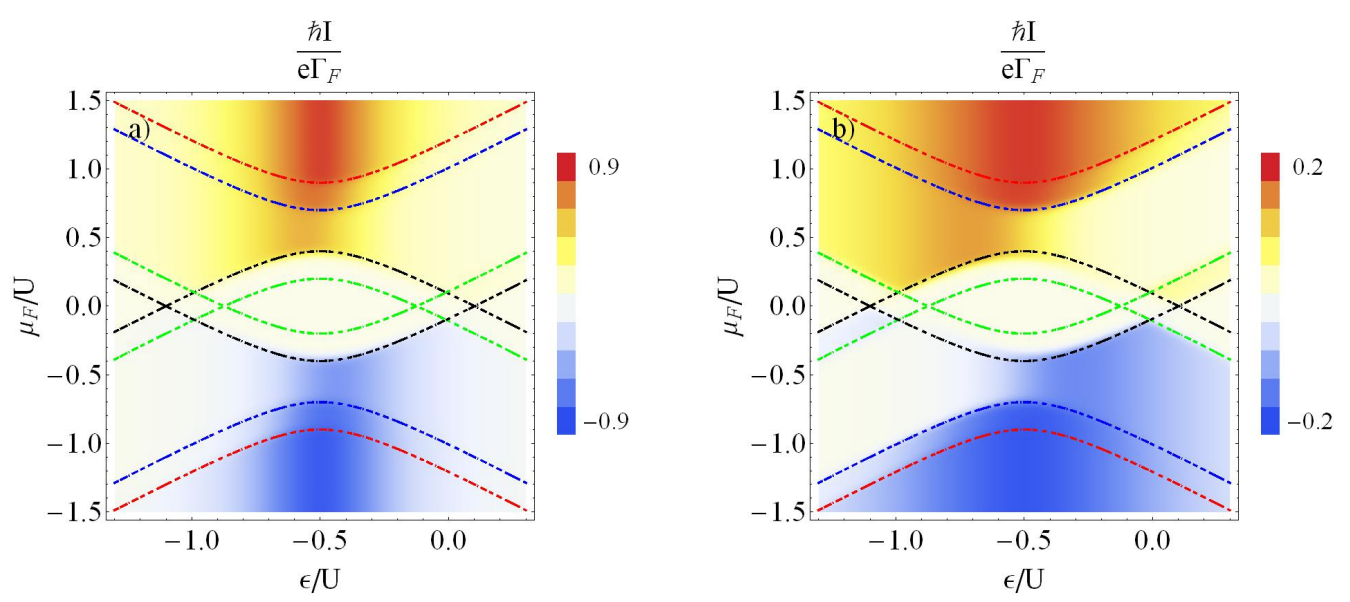

Figure 6.4: Density plots of the current as a function of the chemical potential $\mu_{N}$ and the level position $\epsilon$ for (a) $P=0.4$ and (b) $P=0.9$. The dashed lines show the excitation energies. The red lines show $E_{S+}$, the black lines show $E_{S_{-}}$, the blue lines show $E_{T+}$ and the green lines show $E_{T-}$. Note that the colour scales for the two plots are different. The other parameters used in these plots are $\Gamma_{S}=0.5 U, \beta=50 / U$, $J=0.2 U$ and $B_{1}=B_{2}=0 U$.

different bias voltages and level positions. This is best seen when the polarisation difference is large. Comparing Fig. 6.4 a) and b) we see that the current becomes more asymmetric about $\delta=0$ with increasing polarisation, and that between the $E_{S-}$ and the $E_{T-}$ excitation energies current peaks develop near $\epsilon=-1 U$ and $\epsilon=0 U$. Looking at the differential conductance plots (Fig. 6.5) we can see that there are now negative resonance peaks along some parts of the excitation energies, whereas with $P=0$, for any level position, the current was monotonically increasing with $\mu_{N}$. The probability plots (Fig. 6.6 and 6.7) show that in the regions corresponding to the negative differential conductances the probability of the singlet state decreases and the probabilities of the $|- \pm\rangle$ states become finite, making transport in these regions possible. In the differential conductance plots we also see that the conductance resonances deviate from the excitation energies where the $E_{T-}$ and the $E_{S-}$ lines cross. This is a finite temperature effect that decreases when the temperature is lowered. It is caused by competition between the exponential suppression of components of the generalised transition rates due to temperature and linear suppression due to the polarisation. If the latter effect is greater then we can see differential conductance features that deviate from the excitation energies. Plotting the differential conductance at lower temperatures than that used in Fig. 6.5 would show less deviation from the excitation energies. 

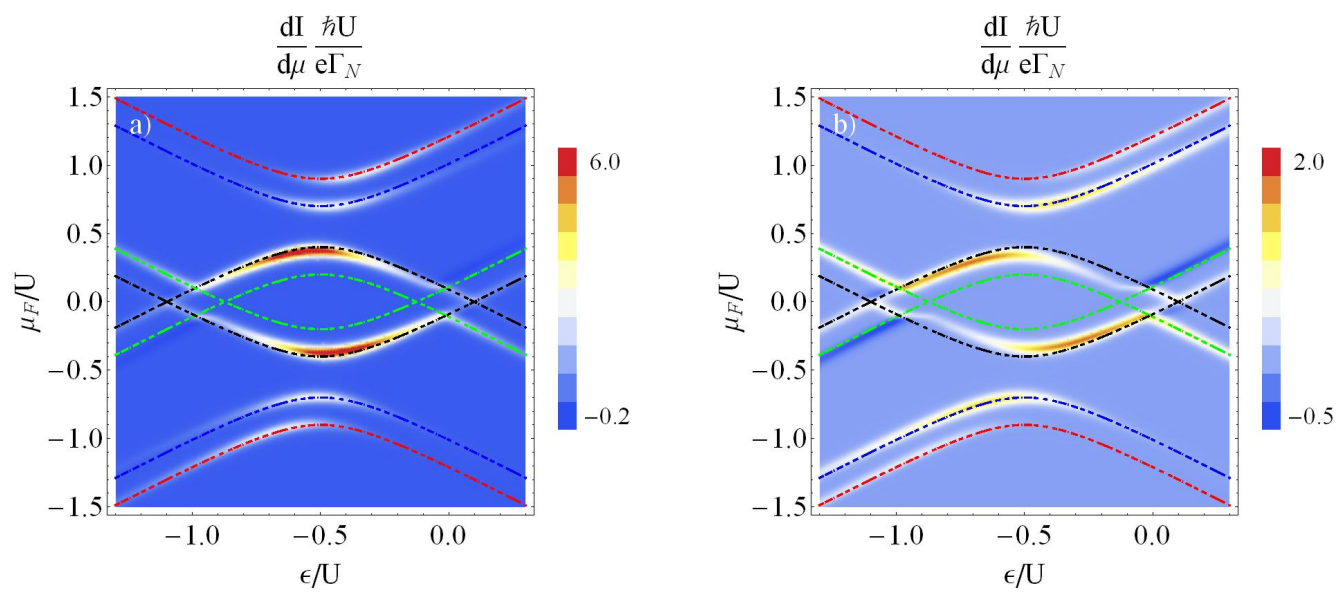

Figure 6.5: Density plots of the differential conductance as a function of the chemical potential $\mu_{N}$ and the level position $\epsilon$ for (a) $P=0.4$ and (b) $P=0.9$. The dashed lines show the excitation energies as in Fig. 6.4. The other parameters used in these plots are $\Gamma_{S}=0.5 U, \beta=50 / U, J=0.2 U$ and $B_{1}=B_{2}=0 U$.

Even though the triplet states and the pairs of Andreev bound states are degenerate, they exhibit different behviour when coupled to the ferromagnetic lead. For example, if the bias and polarisation are both positive then, compared with $|T-\rangle$, transport would be suppressed if the initial state is $|T+\rangle$, as there is a lower density of states of spin down electrons in the lead and therefore a greater waiting time for a spin down electron to tunnel on to the molecule. Due to this the occupation probabilities for all eight states are now different. Figures 6.6 and 6.7 show the occupation probabilities for $P=0.4$ and $P=0.9$, respectively. Plots a) and b) show that for $|T+\rangle$ non-zero probabilities are mainly confined to the $\mu_{F}>0$ half plane, whereas for $|T-\rangle$ the occupation probability is non-zero in the $\mu_{F}<0$ half plane. This can be understood by considering the transport sequence described above. For $\mu_{F}>0$ the molecule will spend more time in the $|T+\rangle$ state as it is less likely for a spin down electron to tunnel on to the molecule. For $\mu_{F}<0$ electrons tunnel from the molecule on to the ferromagnetic lead. If the molecule is initially in one of the bound states then the transfer of a spin up electron to the ferromagnetic lead will be more likely, leaving the molecule occupied by a spin down electron. When the polarisation is negative the density of states of the spin down electrons is greater and the situation described above is reversed. Compared to positive $P$ the occupation probabilities of $|T+\rangle$ and $|T-\rangle$, and $| \pm+\rangle$ and $| \pm-\rangle$ are switched. The probabilities of $|T 0\rangle$ and $|S\rangle$ remain unchanged as they involve both electronic spin orientations. Therefore the current is even in $P$. 

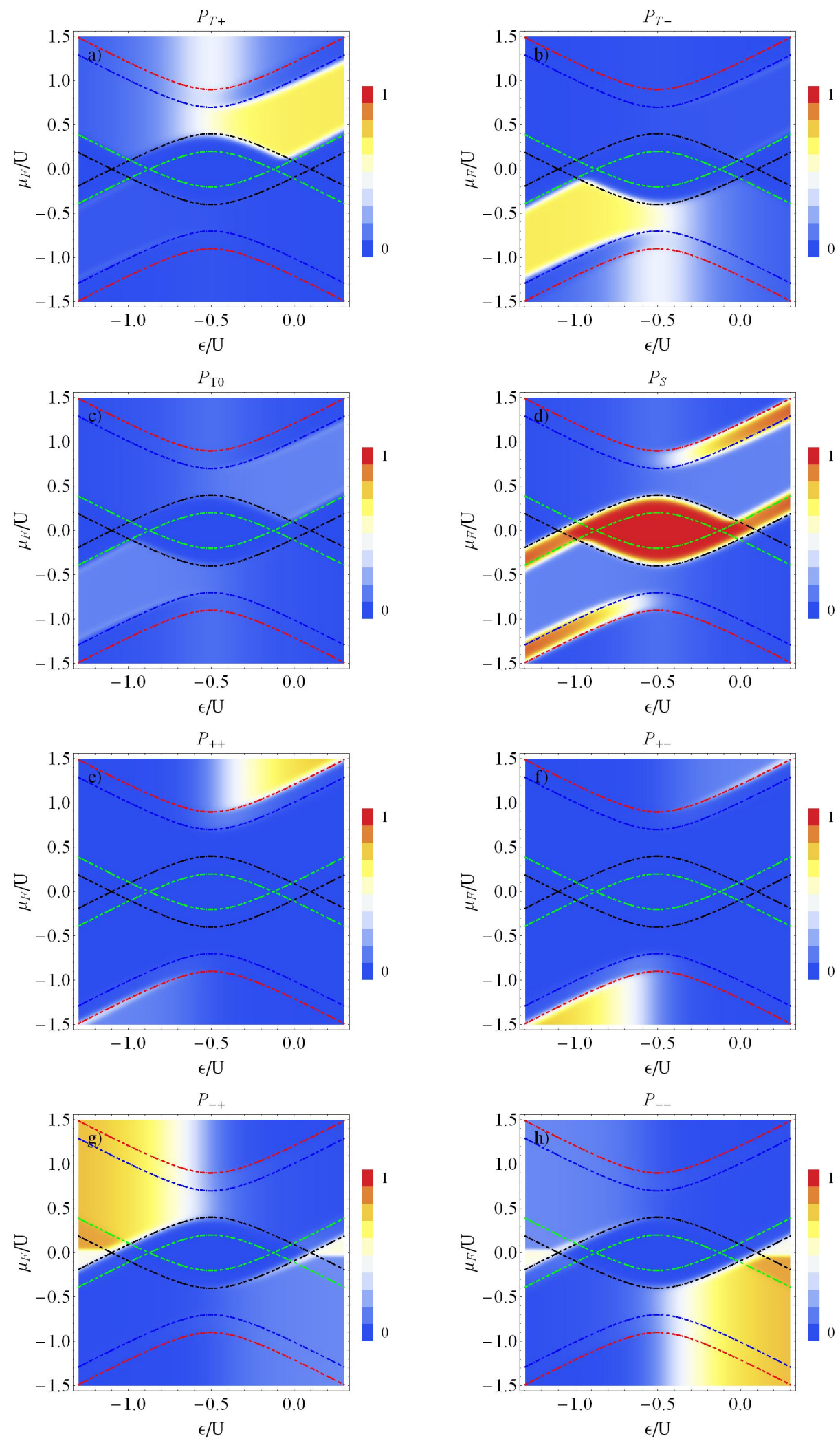

Figure 6.6: Density plots of the occupation probabilities of the eigenstates of $H_{\text {eff }}$ as functions of the chemical potential $\mu_{N}$ and the level position $\epsilon$ for $P=0.4$. The dashed lines show the excitation energies as in Fig. 6.4. The other parameters used in these plots are $\Gamma_{S}=0.5 U, \beta=50 / U, J=0.2 U$ and $B_{1}=B_{2}=0 U$. 

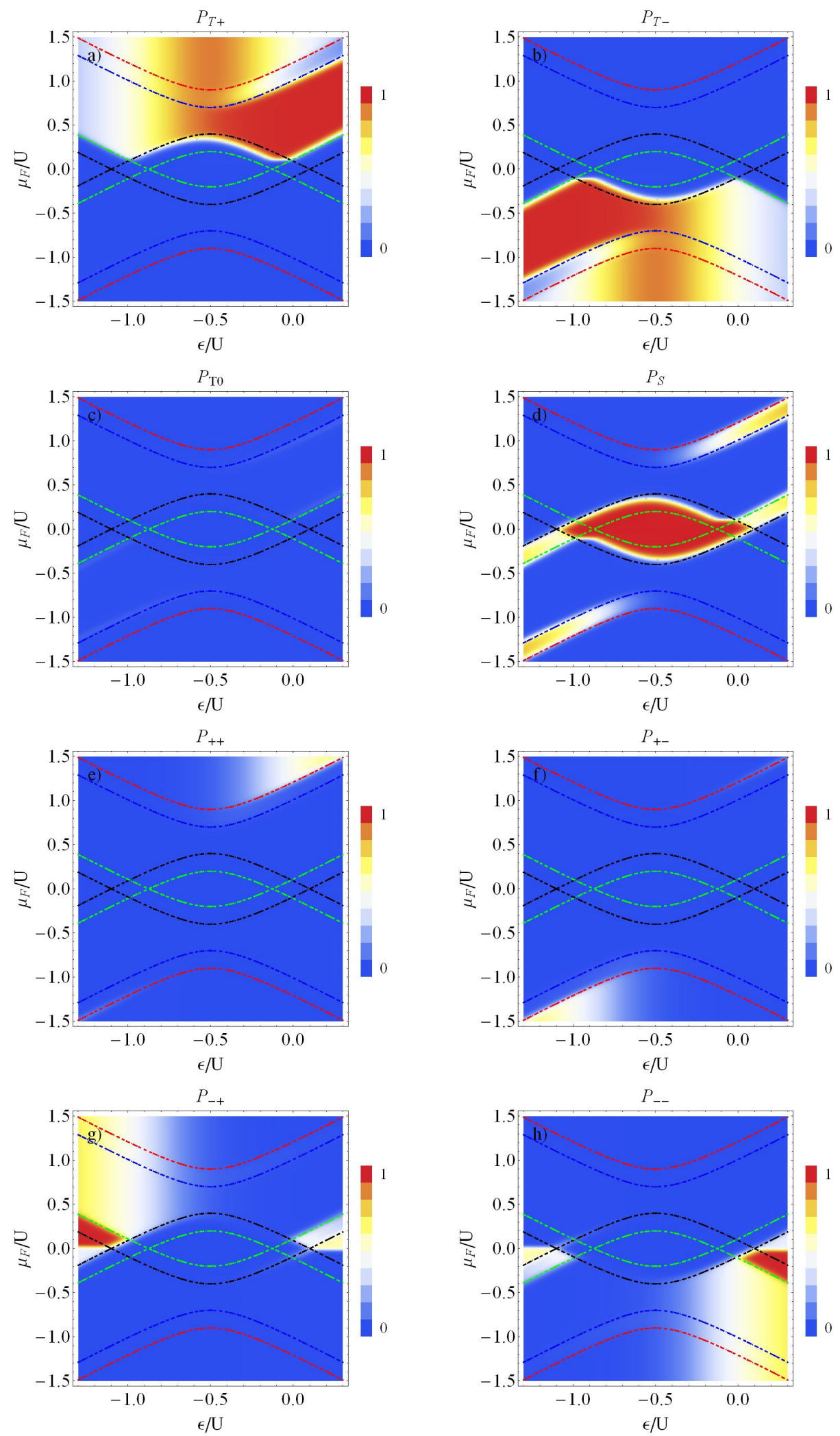

Figure 6.7: Density plots of the occupation probabilities of the eigenstates of $H_{\text {eff }}$ as functions of the chemical potential $\mu_{N}$ and the level position $\epsilon$ for $P=0.9$. The dashed lines show the excitation energies as in Fig. 6.4. The other parameters used in these plots are $\Gamma_{S}=0.5 U, \beta=50 / U, J=0.2 U$ and $B_{1}=B_{2}=0 U$. 

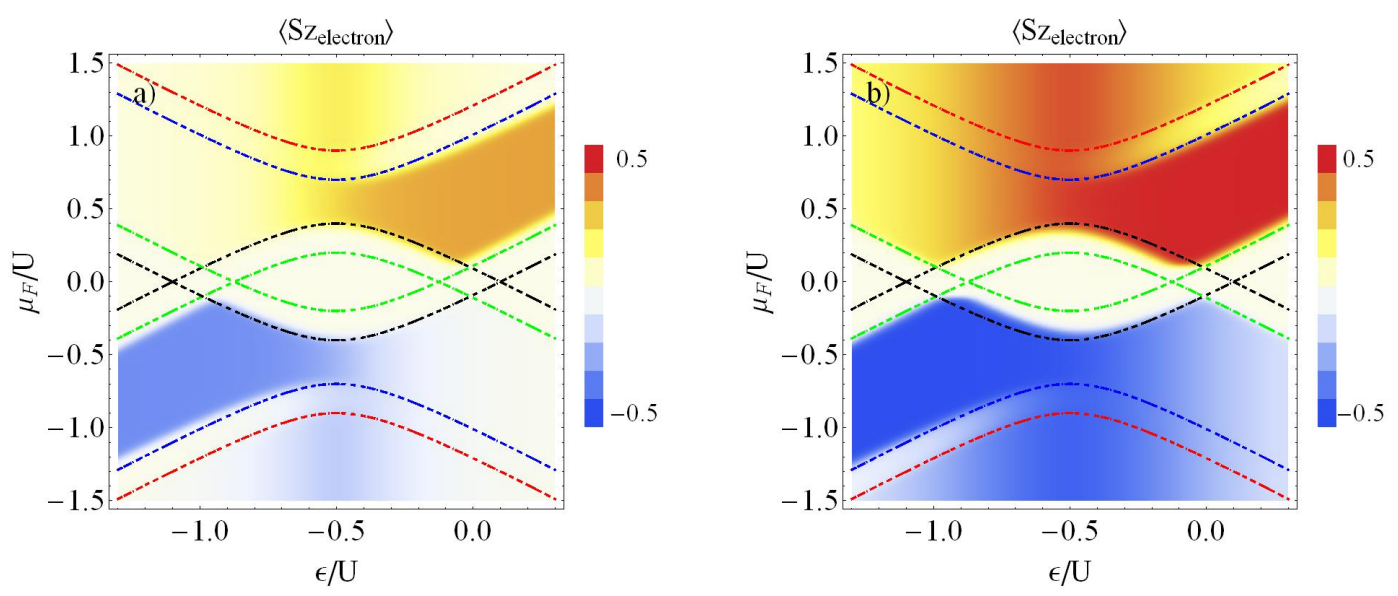

Figure 6.8: Density plots of the expectation value of the $z$ component of the spin of the electrons occupying the orbital of the molecule as a function of the chemical potential $\mu_{N}$ and the level position $\epsilon$ for (a) $P=0.4$ and (b) $P=0.9$. The dashed lines show the excitation energies as in Fig. 6.4. The other parameters used in these plots are $\Gamma_{S}=0.5 U, \beta=50 / U, J=0.2 U$ and $B_{1}=B_{2}=0 U$.
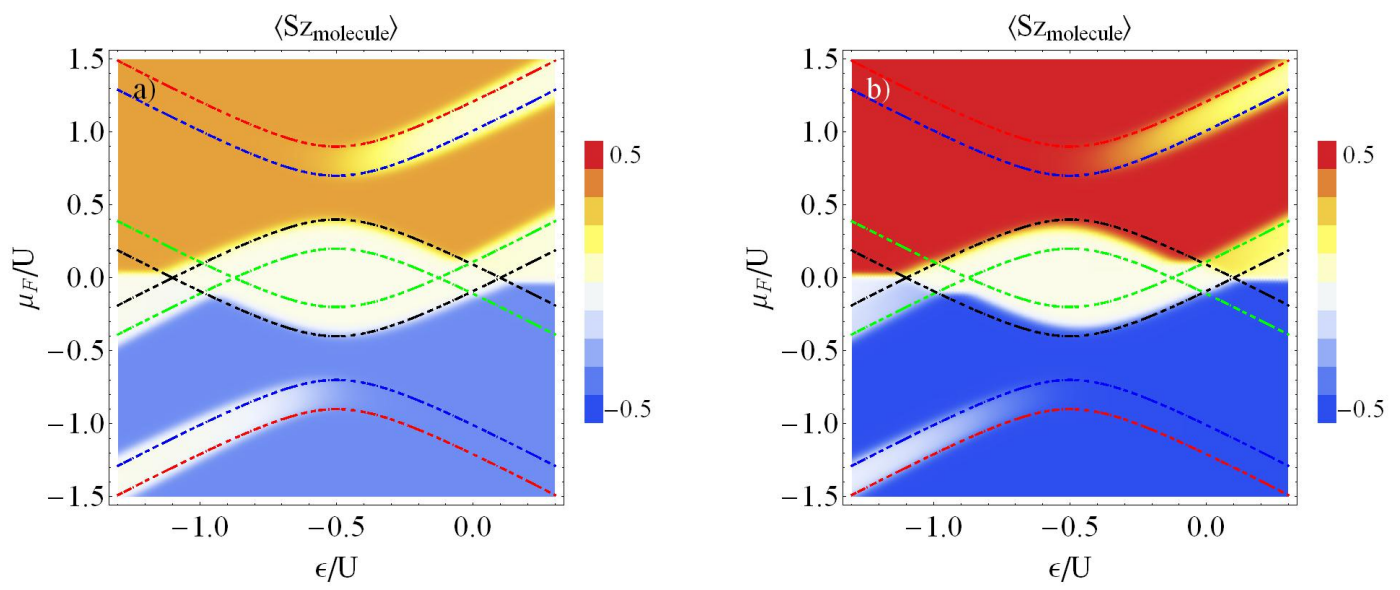

Figure 6.9: Density plots of the expectation value of the $z$ component of the spin of the molecule as a function of the chemical potential $\mu_{N}$ and the level position $\epsilon$ for (a) $P=0.4$ and (b) $P=0.9$. The dashed lines show the excitation energies as in Fig. 6.4. The other parameters used in these plots are $\Gamma_{S}=0.5 U, \beta=50 / U, J=0.2 U$ and $B_{1}=B_{2}=0 U$. 
Due to the eigenstates of the effective Hamiltonian having different occupation probabilities when coupled to the ferromagnetic lead, the expectation values of the electronic and molecular spins are no longer generally zero. Figures 6.8 and 6.9 show the $z$ components of the spin expectation values for $P=0.4$ and $P=0.9$. The expectation values of the $x$ and $y$ components of the spins are still zero. The plots show that for $P>0$ the expectation values of both spins are positive in the upper half plane and negative in the lower half plane. In regions of the plots that correspond to the high occupation probabilities for $|S\rangle$ and $|T 0\rangle$ the expectation values are zero, as these states describe a coherent superposition of spin up and down for both the electronic and molecular spins. For negative polarisation the results are reversed.

To investigate the effect that the polarisation of the ferromagnetic lead has on the superconducting proximity effect we have plotted the normalised current in Fig. 6.10 as a function of the level position. The plot is made for a sufficiently high bias that all conductance channels are open. In Chapter 3 we found that at sufficiently high bias the width of the current peak at half maximum is equal to $\Gamma_{S}$. Here we see that this is no longer the case for $P \neq 0$, for larger polarisations the width of the current peak increases. This means that due to the polarisation of the ferromagnetic lead the current is suppressed, but comparatively less away from zero detuning. Related to this may be the change in the Fano factor for $P \neq 0$. For the system of Chapter 3, at high bias, the Fano factor is equal to 2 when the superconducting proximity effect is off resonance and 1 when it is on resonance. In this case we find no evidence of Poissonian transport on or off resonance. Figure 6.11 shows the Fano factor for $P=0.8$. At zero detuning the Fano factor is equal to approximately 1.6. This is probably because the waiting times for spin up and spin down electrons are different, leading to the distribution of tunneling events being non-Poissonian. 


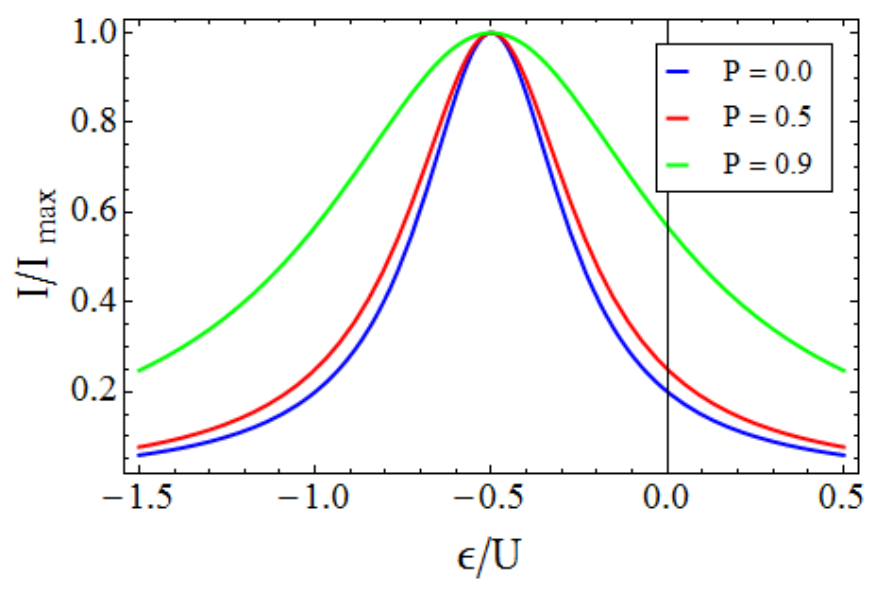

Figure 6.10: Plot of the normalised current as a function of the level position $\epsilon$ for $\mu_{F}=2 U$. The other parameters used in this plot are $\Gamma_{S}=0.5 U, \beta=50 / U, J=0.2 U$ and $B_{1}=B_{2}=0 U$.

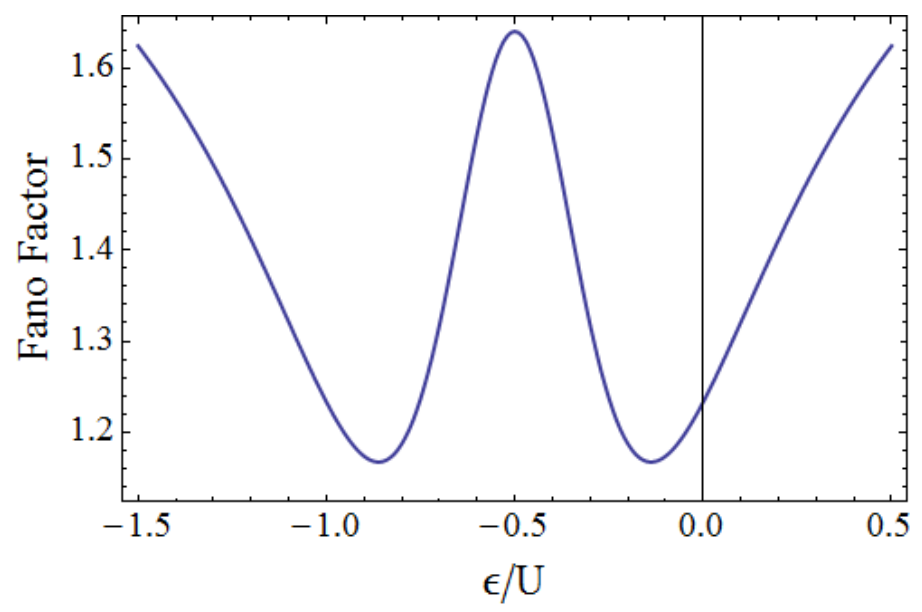

Figure 6.11: Plot of the Fano factor as a function of the level position $\epsilon$ for $\mu_{F}=2 U$ and $P=0.8$. The other parameters used in this plot are $\Gamma_{S}=0.5 U, \beta=50 / U, J=0.2 U$ and $B_{1}=B_{2}=0 U$. 


\subsubsection{External Magnetic Field, $\mathrm{P}=0$}

With an external magnetic field applied to the system the previously degenerate states are split. In the model we use we allow for the electrons and the spin of the molecule to have different $g$-factors. We will first consider the case where the $g$-factors are the same, $B_{1}=B_{2}=B$.

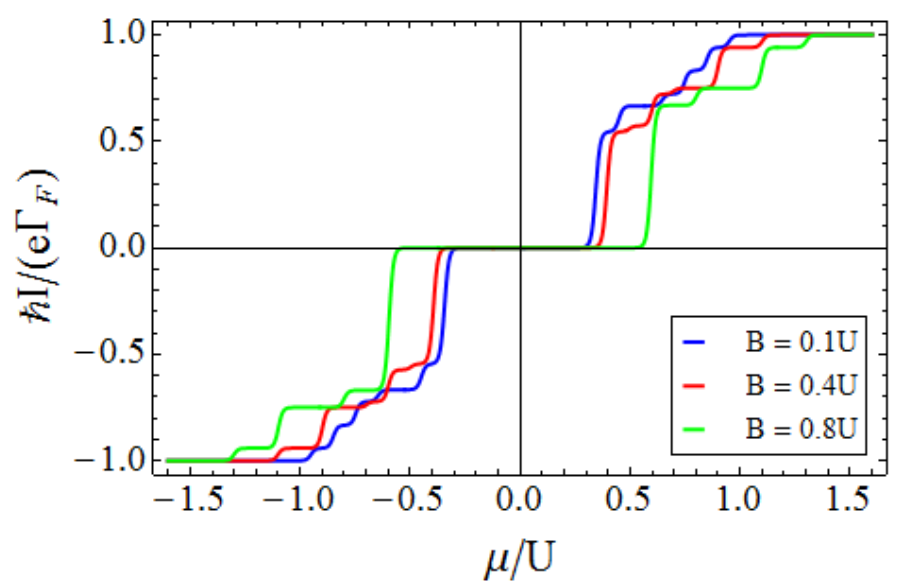

Figure 6.12: Plot of the current as a function of the chemical potential $\mu_{N}$ at $\epsilon=-0.5 U$, for three values of the Zeeman splitting. The other parameters used in this plot are $\Gamma_{S}=0.5 U, \beta=100 / U, J=0.2 U$ and $P=0$.

The splitting of the eigenstates leads to more steps in the current as the voltage is increased, this is shown in Fig. 6.12. For large $B$ the voltage required to initiate transport increases and a higher voltage is needed to reach the maximum current. The maximum current is the same as in the $B=0$ case and density plots of the current have the same form. Figure 6.13 shows a plot of the differential conductance. Here we see that the external magnetic field causes negative features.

Because none of the eigenstates are degenerate in the presence of an external magnetic field the occupation probabilities of the eigenstates are different. This leads to the spin expectation values being non-zero for some parameter values. Figures 6.14 and 6.15 show density plots of the $z$ components of the electronic and molecular spin expectation values for a small and a large external field. In plot a) of both figures the expectation value is zero in the central region, this is because for $B=0.1 U$ the state $|I S\rangle$ is the equilibrium ground states of the molecule. But for $B=0.8 U$ the equilibrium ground state is $|I-\rangle$ and therefore in plot b), for both figures, the expectation value is -0.5 , corresponding to both the electronic and molecular spins being in the down state. If the field is reversed and $B>J$ then the expectation values become positive. 


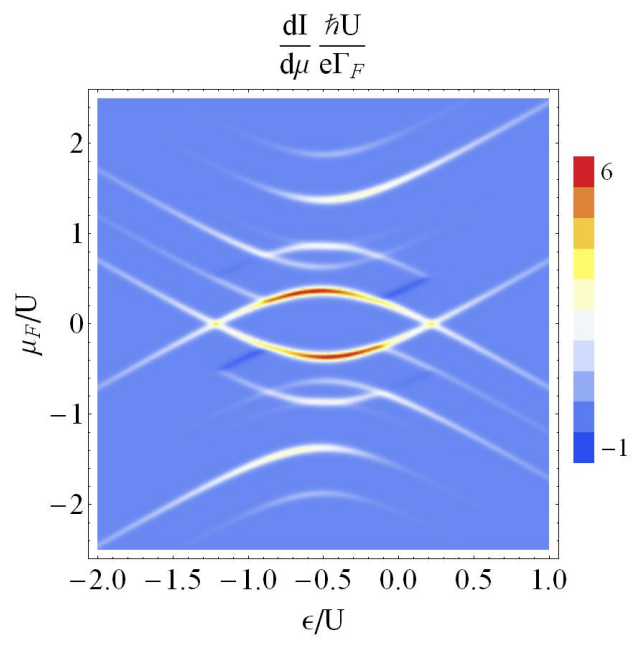

Figure 6.13: Density plot of the differential conductance as a function of the chemical potential $\mu_{N}$ and the level position $\epsilon$. The other parameters used in this plot are $\Gamma_{S}=0.5 U, \beta=50 / U, J=0.5 U, P=0$ and $B=1 U$.

Now we will consider the case where $B_{1}$ and $B_{2}$ are different. For simplicity we will take one to be zero and the other non-zero. Figures 6.16 and 6.17 show plots of the spin expectation values for $B_{1}=0.4 U, B_{2}=0 U$ and $B_{1}=0 U, B_{2}=0.4 U$. For positive $B_{1}$ the non-zero electron spin expectation values are negative and the non-zero molecular spin expectation values are positive. The opposite is true for $B_{2}$ non-zero. This indicates that it is mainly $|I S\rangle$ or $|I 0\rangle$ contributing to the non-zero spin expectation values.

Figure 6.18 shows example plots of the current and the differential conductance for $B_{1}$ and $B_{2}$ not equal and non-zero. As with the N-IMM-S system of Chapter 3 there are features along some of the excitation energies and the current is greatest where the superconducting proximity effect is in resonance. When the bias is high enough that all excitations are possible, the external magnetic field has no effect on the current. The current peak, as a function of $\epsilon$, still has width $\Gamma_{S}$ and height $\Gamma_{F}$. The Fano factor also shows the same behaviour as for the N-IMM-S system. When the superconducting proximity effect is off resonance the Fano factor is 2; on resonance it is 1 . 

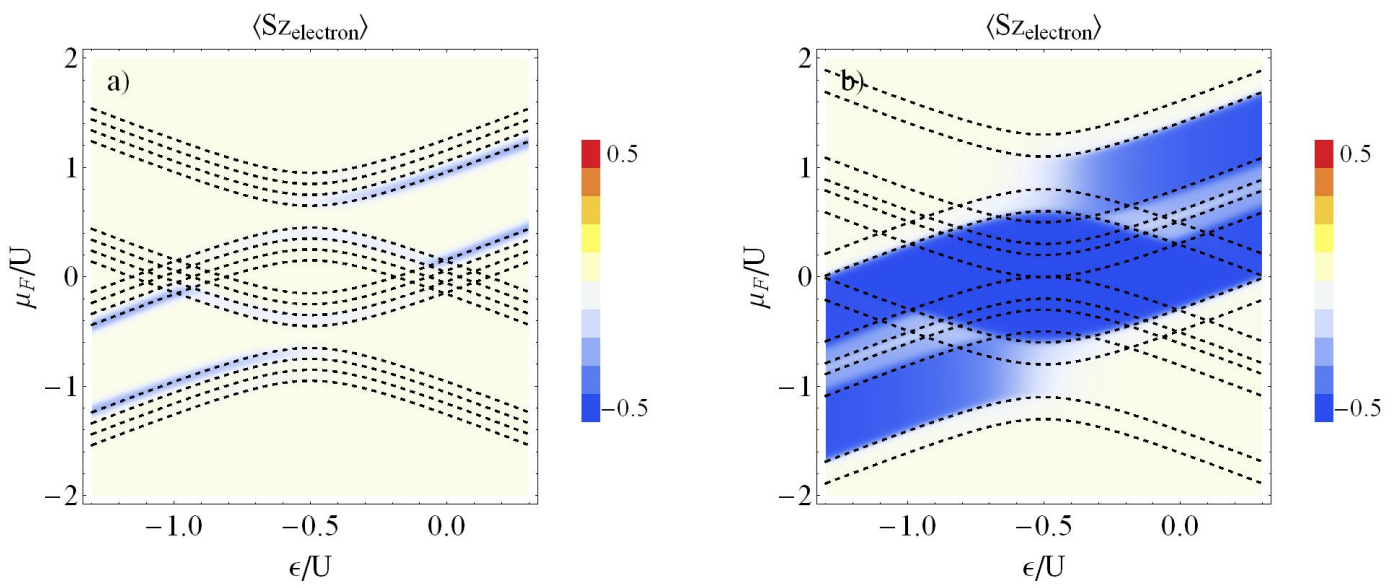

Figure 6.14: Density plots of the expectation value of the $z$ component of the spin of the electrons occupying the orbital of the molecule as a function of the chemical potential $\mu_{N}$ and the level position $\epsilon$ for (a) $B=0.1 U$ and (b) $B=0.8 U$. The dashed lines show the excitation energies. The other parameters used in these plots are $\Gamma_{S}=0.5 U$, $\beta=50 / U, J=0.2 U$ and $P=0$.
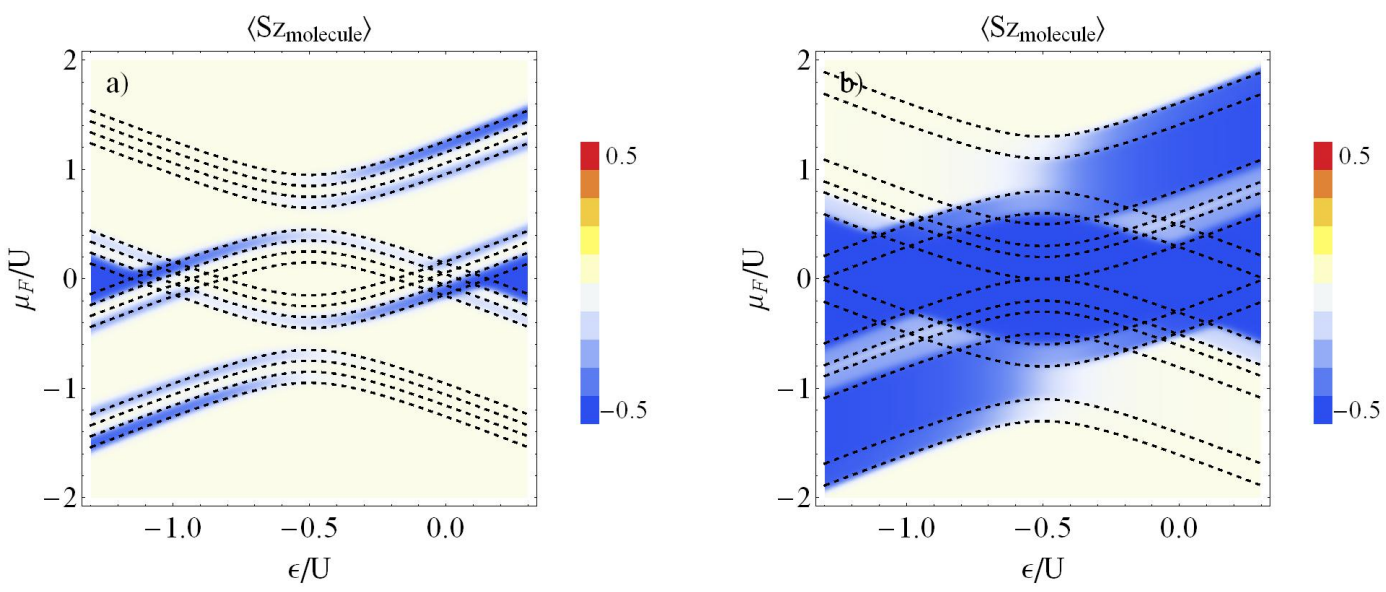

Figure 6.15: Density plots of the expectation value of the $z$ component of the spin of the molecule as a function of the chemical potential $\mu_{N}$ and the level position $\epsilon$ for (a) $B=0.1 U$ and (b) $B=0.8 U$. The dashed lines show the excitation energies. The other parameters used in these plots are $\Gamma_{S}=0.5 U, \beta=50 / U, J=0.2 U$ and $P=0$. 

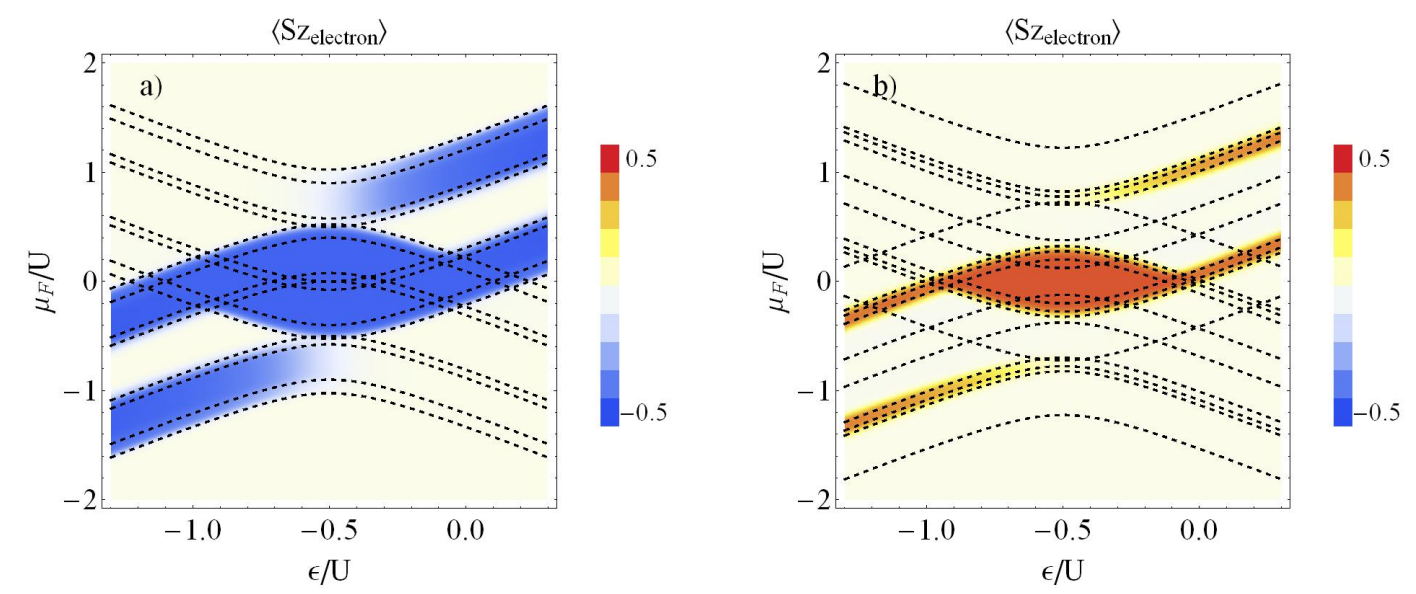

Figure 6.16: Density plots of the expectation value of the $z$ component of the spin of the electrons occupying the orbital of the molecule as a function of the chemical potential $\mu_{N}$ and the level position $\epsilon$ for (a) $B_{1}=0.4 U, B_{2}=0 U$ and (b) $B_{1}=0 U, B_{2}=0.4 U$. The dashed lines show the excitation energies. The other parameters used in these plots are $\Gamma_{S}=0.5 U, \beta=50 / U, J=0.2 U$ and $P=0$.
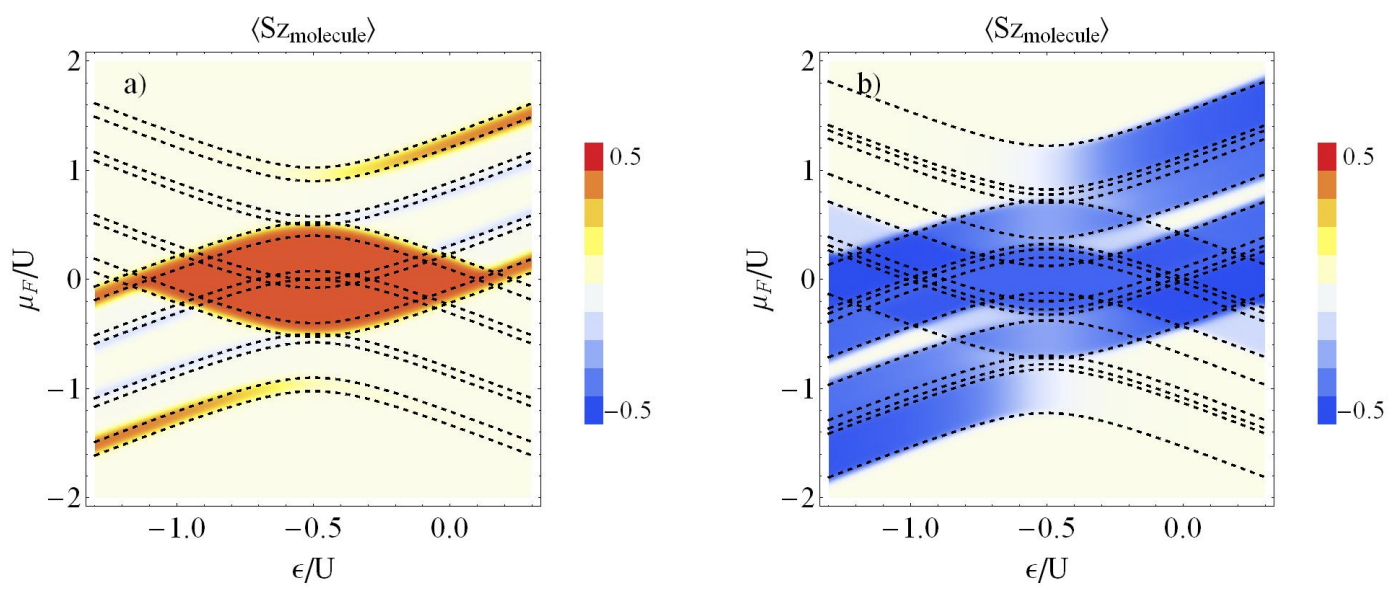

Figure 6.17: Density plots of the expectation value of the $z$ component of the spin of the molecule as a function of the chemical potential $\mu_{N}$ and the level position $\epsilon$ for (a) $B_{1}=0.4 U, B_{2}=0 U$ and (b) $B_{1}=0 U, B_{2}=0.4 U$. The dashed lines show the excitation energies. The other parameters used in these plots are $\Gamma_{S}=0.5 U, \beta=50 / U, J=0.2 U$ and $P=0$. 

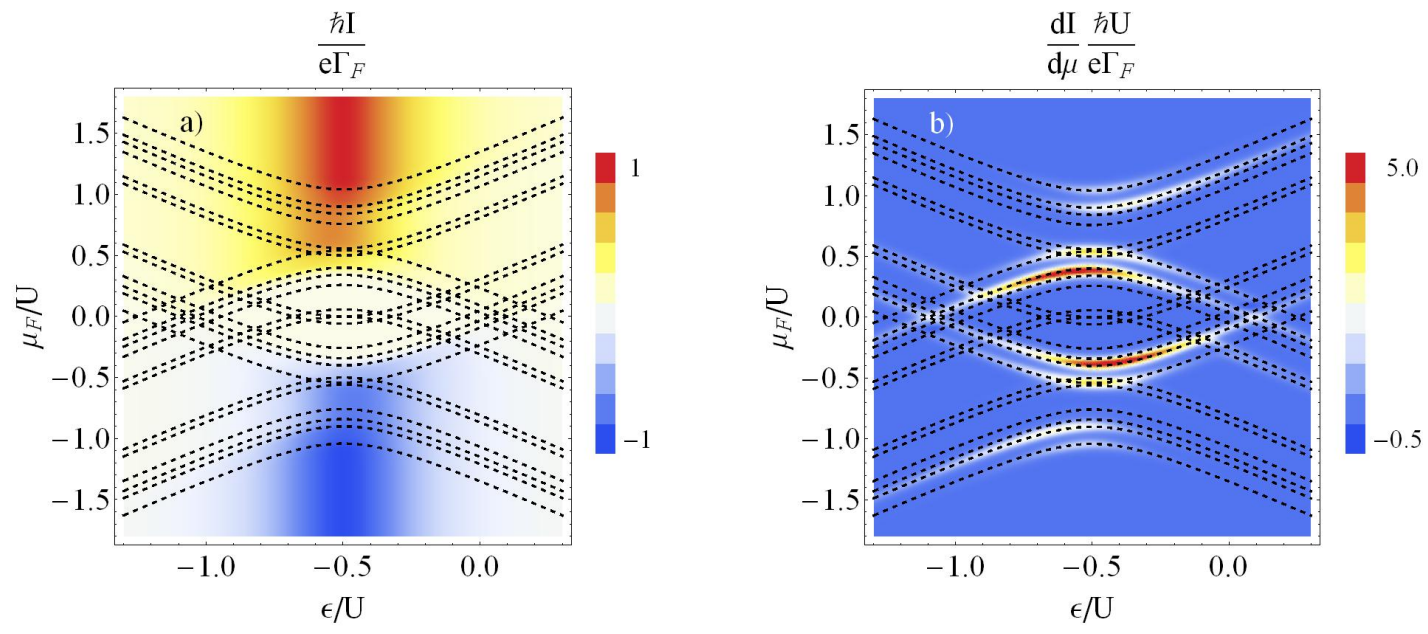

Figure 6.18: Density plots of (a) the current and (b) the differential conductance as functions of the chemical potential $\mu_{N}$ and the level position $\epsilon$ for $B_{1}=0.4 U$ and $B_{2}=0.2 U$. The dashed lines show the excitation energies. The other parameters used in these plots are $\Gamma_{S}=0.5 U, \beta=50 / U, J=0.2 U$ and $P=0$.

\subsubsection{Ferromagnetic Lead and External Magnetic Field}

When we include both the polarisation of the lead and the external magnetic field the current and spin expectation value density plots show features that are combinations of what is discussed above. The maximum current is restricted by the polarisation of the lead and the features in the current and differential conductance plots can be seen near the excitation energies. At which excitation energies current features can be observed again depends on the configuration of the excitation energies, as discussed in Chapter 3. Shown in Fig. 6.19 are two examples of current density plots. When there is no external magnetic field the current is even in $P$, however when there is an external magnetic field this is no longer the case. This is because in the presence of an external field the formerly triplet states are no longer degenerate. With either $B_{1}=B_{2}=0$ or $P=0$ the current is antisymmetric about $\delta=0, \mu_{F}=0$, however this is not the case when both the polarisation and the external magnetic field are non-zero (Fig. 6.19). With only $P$ non-zero we found that one of $|T \pm\rangle$ mainly contributed to the current for positive bias and the other for negative bias. Because these states have the same energy there is a symmetry about $\mu_{F}=0$. But when the external field is also non-zero then the corresponding eigenstates, $|I \pm\rangle$, do not have the same energy and therefore the symmetry about $\mu_{F}=0$ is broken. 

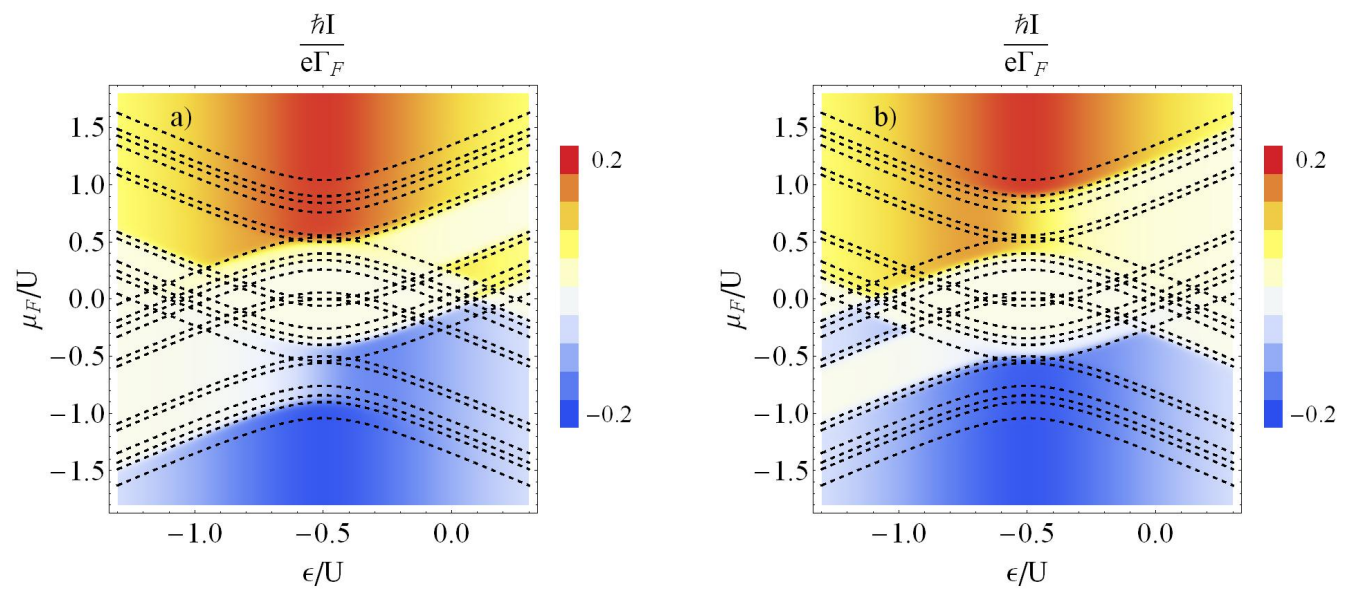

Figure 6.19: Density plots of the current as a function of the chemical potential $\mu_{N}$ and the level position $\epsilon$ for (a) $P=0.9$ and (b) $P=-0.9$. The dashed lines show the excitation energies. The other parameters used in these plots are $\Gamma_{S}=0.5 U, \beta=50 / U$, $J=0.2 U, B_{1}=0.4 U$ and $B_{2}=0.2 U$.

Figure 6.20 shows the differential conductance for $P=0.8, B_{1}=0.4 U$ and $B_{2}=0.2 U$. Comparing this plot to Fig. $6.18 \mathrm{~b}$ ) we see that the introduction of the polarisation of the lead has vastly changed the differential conductance resonances. We see again the symmetry about $\delta=0, \mu_{F}=0$ is no longer present. The magnitude of the resonances have also decreased. This is because the current decreases with increasing $|P|$, but the width of the current steps depend on $k_{B} T$. Some features that were visible in Fig. 6.18 b) are not present in Fig. 6.20. This can be attributed to the change in occupation probability for $P=0$ compared with $P=0.8$. For example in Figs. 6.6 and 6.7 we saw the probability of $|T 0\rangle$ is suppressed with increasing $|P|$. Furthermore, we see prominent negative differential conductance features for $P \neq 0$.

The effects of $J, \Gamma_{S}$ and $k_{B} T$ are the same as in Chapter $3 . \Gamma_{S}$ controls the width of the current peak about $\delta=0 . J$ shifts the excitation energies and controls the size of the central current suppressed region. And, the temperature affects the width of the current steps.

\subsection{Results - Non-collinear}

In this section we present graphical results for the current and the spin expectation values for the non-collinear case. Again, due to the size of the system we were not able to obtain analytic results. Firstly we will investigate the $\Gamma_{F}$ dependence of the 


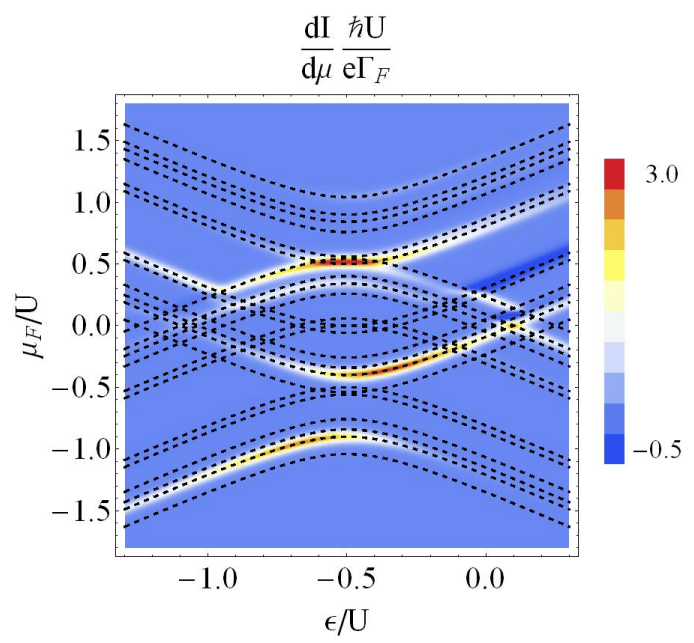

Figure 6.20: Density plots of the differential conductance as a function of the chemical potential $\mu_{N}$ and the level position $\epsilon$ for $B_{1}=0.4 U, B_{2}=0.2 U$ and $P=0.8$. The dashed lines show the excitation energies. The other parameters used in this plot are $\Gamma_{S}=0.5 U$, $\beta=50 / U$ and $J=0.2 U$.

current and the error induced by the inclusion of off-diagonal reduced density matrix elements. Then we will investigate the effect of the polarisation and orientation of the ferromagnetic lead when the off-diagonal reduced density matrix elements are in resonance at zero detuning. Lastly we will look at the effect of shifting the resonances of the off-diagonal reduced density matrix elements.

\subsubsection{Dependence of Current on $\Gamma_{F}$}

In the previous models because we calculated the current to first order in the tunnel coupling to the metallic lead $I \propto \Gamma_{N / F}$. However, due to the inclusion of off-diagonal elements in this model this is no longer the case. To first order in $\Gamma_{F}$ the off-diagonal density matrix elements $P_{\chi^{\prime}}^{\chi}$ are non-zero if $\Delta E=E_{\chi}-E_{\chi^{\prime}} \lesssim \Gamma_{F}$. Rewriting Eq. 2.20 in terms of the vector, $\overline{\mathbf{W}}$, comprised of the generalised transition rates that end in an off-diagonal term and the vector, $\overline{\mathbf{P}}$, containing the corresponding non-zero reduced density matrix elements we get

$$
P_{\chi^{\prime}}^{\chi}=\frac{-i}{\Delta E} \overline{\mathbf{W}} \cdot \overline{\mathbf{P}}
$$


Rearranging for $P_{\chi^{\prime}}^{\chi}$ gives

$$
P_{\chi^{\prime}}^{\chi}=\frac{-\overline{\mathbf{W}}^{\prime} \cdot \overline{\mathbf{P}}^{\prime}\left(i+\frac{1}{\Delta E} W_{\chi^{\prime} \chi^{\prime}}^{\chi \chi}\right)}{\Delta E\left(1+\left(\frac{1}{\Delta E} W_{\chi^{\prime} \chi^{\prime}}^{\chi \chi}\right)^{2}\right)},
$$

where $\overline{\mathbf{W}}^{\prime}$ and $\overline{\mathbf{P}}^{\prime}$ are the previous vectors with the $W_{\chi^{\prime} \chi^{\prime}}^{\chi \chi}$ and $P_{\chi^{\prime}}^{\chi}$ terms removed. The off-diagonal element is on resonance when $\Delta E \rightarrow 0$. In this regime Eq.6.41 becomes

$$
P_{\chi^{\prime}}^{\chi}=\frac{-\overline{\mathbf{W}}^{\prime} \cdot \overline{\mathbf{P}}^{\prime}}{W_{\chi^{\prime} \chi^{\prime}}^{\chi \chi}}
$$

meaning that $P_{\chi^{\prime}}^{\chi}$ is of zeroth order in $\Gamma_{F}$ and therefore the current is proportional to $\Gamma_{F}$. As $\Delta E$ gets larger

$$
P_{\chi^{\prime}}^{\chi} \approx-\frac{i \overline{\mathbf{W}}^{\prime} \cdot \overline{\mathbf{P}}^{\prime}}{\Delta E}-\frac{\overline{\mathbf{W}}^{\prime} \cdot \overline{\mathbf{P}}^{\prime} W_{\chi^{\prime} \chi^{\prime}}^{\chi \chi}}{\Delta E^{2}}
$$

For $\Delta E \gg \Gamma_{F}$ the corresponding off-diagonal reduced density matrix elements should not be included and therefore the current should be proportional to $\Gamma_{F}$. In Fig. 6.21 a) we have plotted the current, in units of $\Gamma_{F}$, as a function of $\Gamma_{F}$ for $\Delta E \approx 0.08 U$. In this plot $\Delta E \gg \Gamma_{F}$ for the entire $\Gamma_{F}$ range that is shown. Here we see that the current is not proportional to $\Gamma_{F}$, but that it changes by less than a $0.02 \%$ for a $\Gamma_{F}$ range of three orders of magnitude. Therefore the erroneous inclusion of the off-diagonal elements has little affect. When $\Delta E \lesssim \Gamma_{F}$ the off-diagonal elements should be included. However as we can see from Eq. 6.43 this means that $P_{\chi^{\prime}}^{\chi}$ will not be of zeroth order in $\Gamma_{F}$, which leads to terms in the current that are of higher order than first. The inclusion of higher order terms in the current is unavoidable when there are off-diagonal density matrix elements, as there is no systematic way to include the first order terms only. However the error introduced due to this is very small and only occurs for a narrow range of parameter values. Figure $6.21 \mathrm{~b}$ ) shows the current for $\Delta E \approx 0.0002 U$ as a function of $\Gamma_{F}$. For the range of values where $\Delta E \lesssim \Gamma_{F}$ we see some change in the current, meaning that it is not proportional to $\Gamma_{F}$, however this change is not significant compared with the magnitude of the current. In the plots of Fig. 6.21 we have only used $\Gamma_{F}$ values as high as $0.001 U$ as we require $\Gamma_{F} \ll k_{B} T$.

Because the current is not proportional to $\Gamma_{F}$ it is necessary to specify its value. However as the change in the current, in units of $\Gamma_{F}$, is very small as $\Gamma_{F}$ is varied the results for a specific $\Gamma_{F}$ value are representative of a large range of $\Gamma_{F}$ values. Thus, in the subsequent sections we ignore the error introduced by the off-diagonal elements 

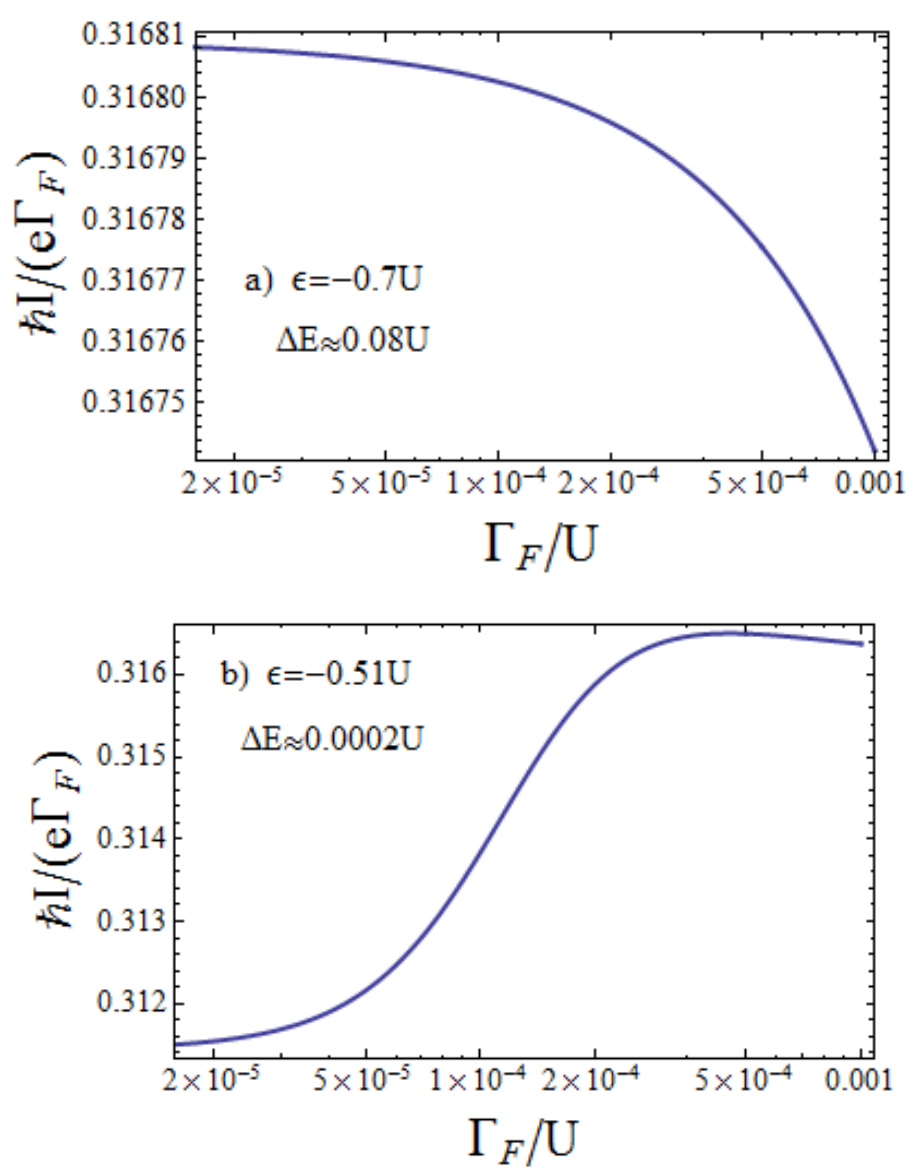

Figure 6.21: Plots of the current as a function of $\Gamma_{F}$ near parameter values where the off-diagonal density matrix elements are in resonance. The parameters used in these plots are $\phi=\pi / 4, k_{B} T=0.05 U, J=0.5 U, B=1 U, \Gamma_{S}=1 U, P=0.8$ and $\mu_{F}=0.7 U$.

and give the current in units of $\Gamma_{F}$, with $\Gamma_{F}=0.001 U$.

\subsubsection{Effect of Polarisation and Alignment of the Magnetisa- tion of the Ferromagnetic Lead - $B=\Gamma_{S}=2 J$}

In this section we will explore the effect of changing the polaristion and the angle of the ferromagnetic lead. Because this system is very similar to that of the previous section and that of Chapter 3 its dynamics should reduce to those of these systems in certain limits. When $\phi=0$ the quantisation axis of the ferromagnetic lead is parallel with the external magnetic field and the current and probabilities reduced to the results given in the previous section. As required the rates connecting off-diagonal reduced density matrix elements are zero in this limit, this is made evident by the factor $\sin \phi$ contained 

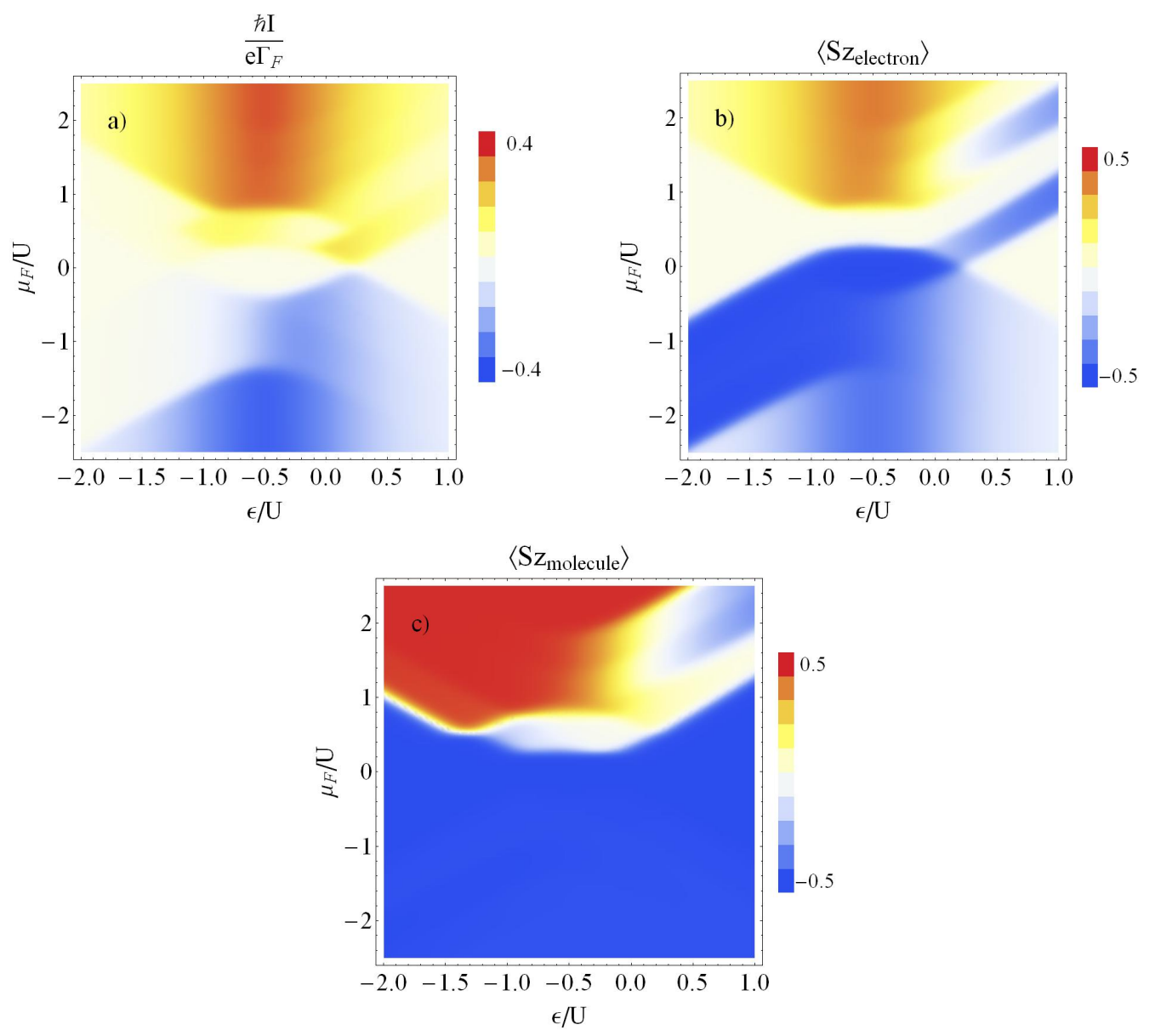

Figure 6.22: Density plots of (a) the current, (b) the $z$ component of the electron spin expectation value and (c) the $z$ component of the molecular spin expectation value as functions of the chemical potential $\mu_{N}$ and the level position $\epsilon$ for $\phi=0$. The other parameters used in these plots are $k_{B} T=0.05 U, J=0.5 U, B=1 U, \Gamma_{S}=1 U, P=0.8$ and $\Gamma_{F}=0.001 U$.

in these rates. When the polarisation is zero then the results are independent of $\phi$ as the lead is non-magnetic. In the first system we did not consider an external magnetic field, therefore this system only reduces to that of Chapter 3 when the external field it set to zero.

In Fig. 6.22 the current and spin expectation values are shown for $\phi=0$. Figures $6.23,6.24$ and 6.25 show the same plots for $\phi=\frac{\pi}{4}, \phi=\frac{\pi}{2}$ and $\phi=\frac{3 \pi}{4}$, respectively. Note that the scales for these plots vary. We have not shown plots of negative angles as $I(\phi)=I(-\phi)$. As $\phi$ increases from 0 to $\frac{\pi}{2}$ the maximum current, which is achieved at high bias and level position $\epsilon=-0.5 U$, increases and peaks at $\phi=\frac{\pi}{2}$. This is also shown 

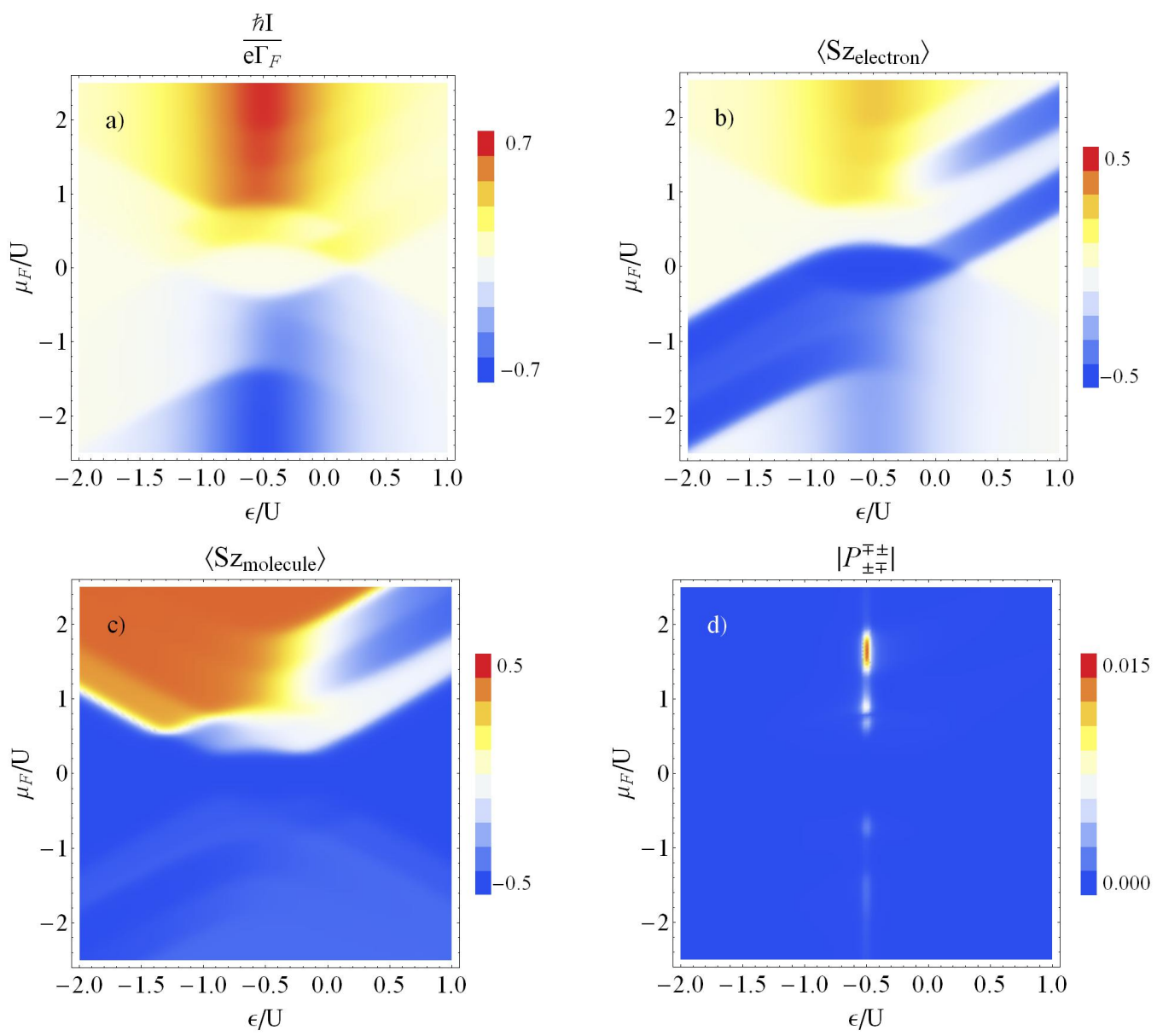

Figure 6.23: Density plots of (a) the current, (b) the $z$ component of the electron spin expectation value, (c) the $z$ component of the molecular spin expectation value and (d) the absolute value of the off-diagonal reduced density matrix elements as functions of the chemical potential $\mu_{N}$ and the level position $\epsilon$ for $\phi=\pi / 4$. The other parameters used in these plots are $k_{B} T=0.05 U, J=0.5 U, B=1 U, \Gamma_{S}=1 U, P=0.8$ and $\Gamma_{F}=0.001 U$.

clearly in Fig. 6.26, which shows the current as a function of $\mu_{F}$ at zero detuning. This can be understood by considering the coefficients of the generalised transition rates. When $\phi=\frac{\pi}{2}$ the rates involving only diagonal reduced density matrix elements reduce to the transition rates for the system with a normal lead coupled to the magnetic molecule. The generalised rates connecting diagonal to off-diagonal reduced density matrix elements all depend on a factor of $\sin \phi$, therefore when $\phi=\frac{\pi}{2}$ these terms are maximal. In the absence of these terms the dynamics of the system would be identical to the N-MM-S system. However, with the off-diagonal elements present there is a difference in the current between the two systems that occurs at $\mu_{F}$ and $\epsilon$ 
values where the off-diagonal elements are non-zero and depends on the size of these elements. Physically it makes sense that the current is greatest when the angle between the magnetistion of the lead and the external magnetic field is $\frac{\pi}{2}$, as at this angle both the majority and the minority carriers have equal coupling strengths for tunneling on to the molecule in a spin up or a spin down state, meaning that transport is not limited by the minority carriers.
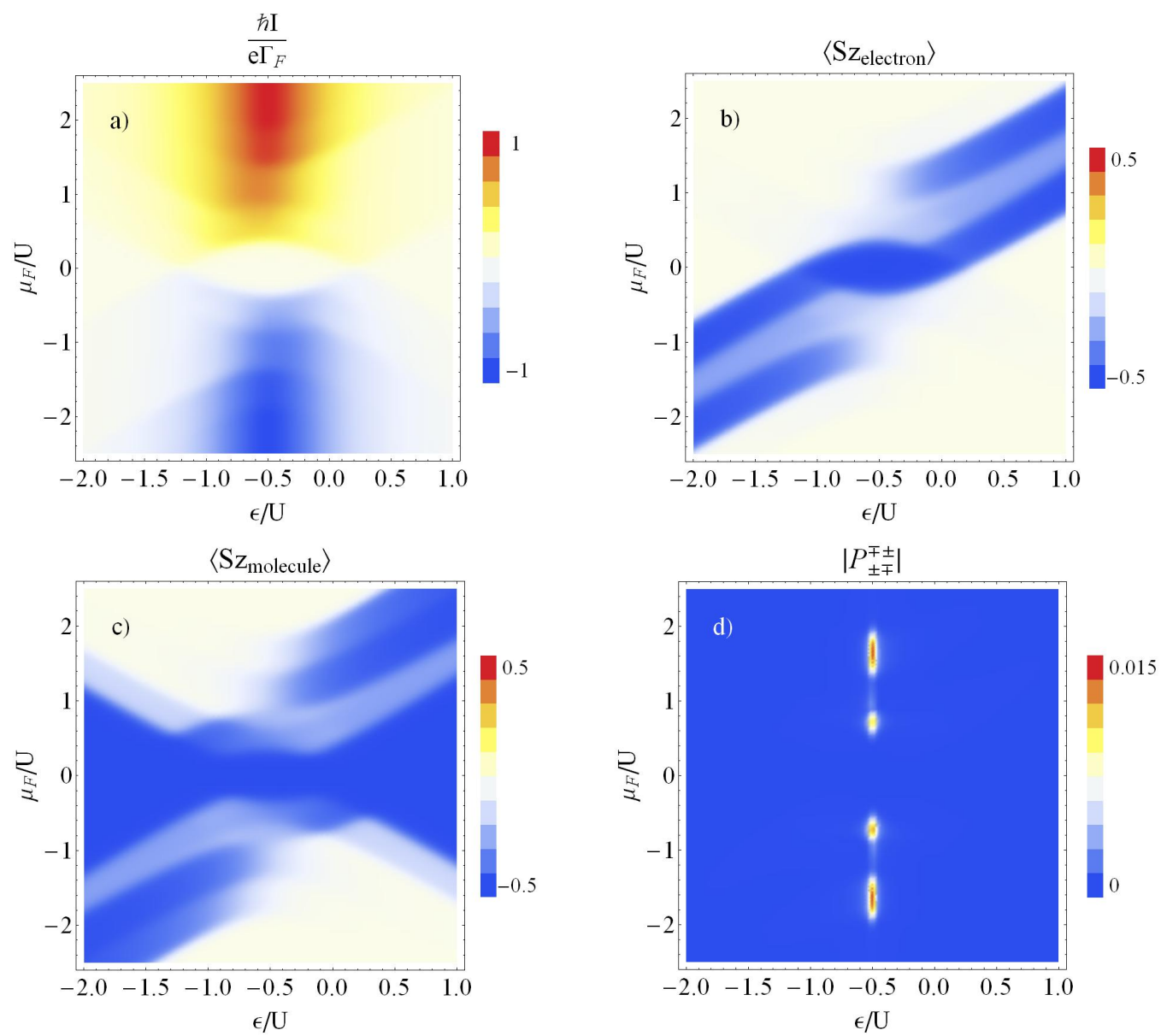

Figure 6.24: Density plots of (a) the current, (b) the $z$ component of the electron spin expectation value, (c) the $z$ component of the molecular spin expectation value and (d) the absolute value of the off-diagonal reduced density matrix elements as functions of the chemical potential $\mu_{N}$ and the level position $\epsilon$ for $\phi=\pi / 2$. The other parameters used in these plots are $k_{B} T=0.05 U, J=0.5 U, B=1 U, \Gamma_{S}=1 U, P=0.8$ and $\Gamma_{F}=0.001 U$.

We have not plotted the excitation energies in Figs. 6.22-6.25, so as to not obscure features of the density plots, however the features in the current and spin expectation value plots are in the vicinity of the excitation energies of the eigenstates. As the angle, 

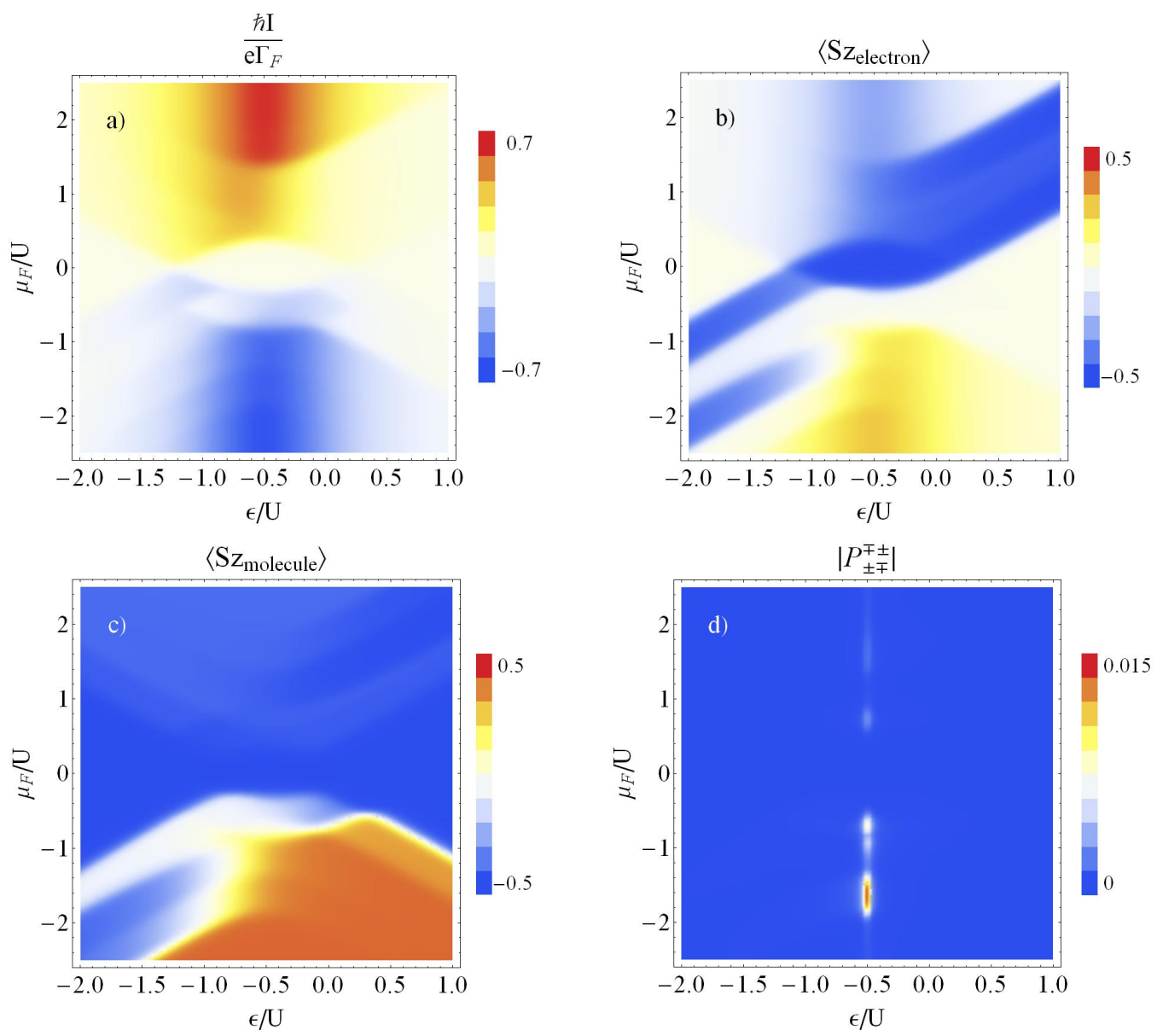

Figure 6.25: Density plots of (a) the current, (b) the $z$ component of the electron spin expectation value, (c) the $z$ component of the molecular spin expectation value and (d) the absolute value of the off-diagonal reduced density matrix elements as functions of the chemical potential $\mu_{N}$ and the level position $\epsilon$ for $\phi=3 \pi / 4$. The other parameters used in these plots are $k_{B} T=0.05 U, J=0.5 U, B=1 U, \Gamma_{S}=1 U, P=0.8$ and $\Gamma_{F}=0.001 U$.

$\phi$, is varied between zero and $\pi$ the locations of the features are unchanged, but their prominence increases or decreases. This can be seen in the density plots of the current, but is made more apparent in Figs. 6.26 and 6.27, which show the current as a function of the electrochemical potential of the ferromagnetic lead for three values of $\epsilon$. The variation of the angle between these limits has this effect on the current because there is no change to what transport channels are open, only the likelihood of each transition is varied, since the tunnel coupling strengths depend on $\phi$.

The expectation values of the $x$ and $y$ components of the molecular and electronic spins are zero. This is because in the regime we are working in there are no coherent 


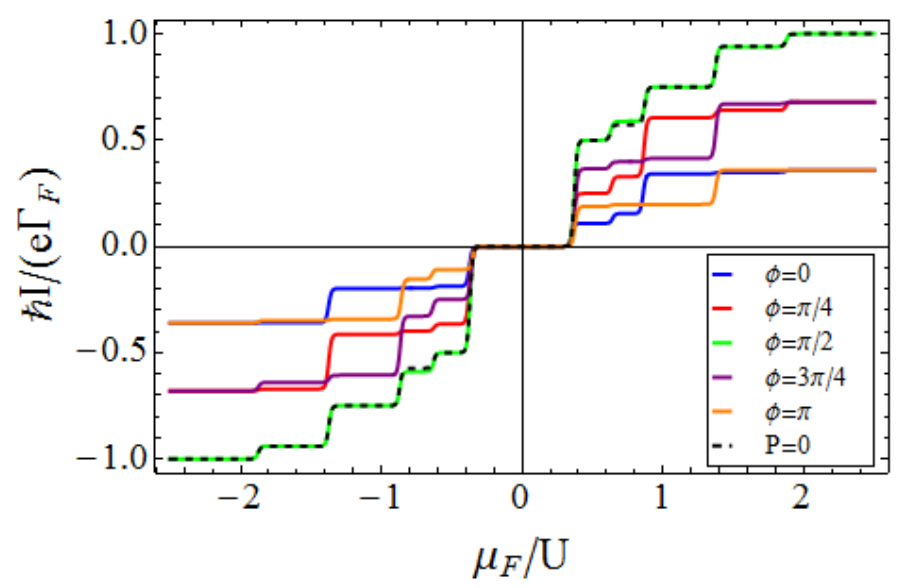

Figure 6.26: Plot of the current as a function of the chemical potential $\mu_{N}$ for various alignments of the magnetisation of the ferromagnetic lead with respect to the external magnetic field. The parameters used in this plot are $J=0.5 U, B=1 U, \Gamma_{S}=1 U$, $P=0.8, \epsilon=-0.5 U$ and $\Gamma_{F}=0.001 U$.

superpositions of states with the same electronic state but different molecular spins (or vice versa). Even though the inclusion of the $P_{+-}^{-+}$and $P_{-+}^{+-}$reduced density matrix elements means there is superposition of molecular spin up and spin down, this does not lead to non-zero $\left\langle S_{x}\right\rangle$ or $\left\langle S_{y}\right\rangle$ because the parts of the $| \pm, \mp\rangle$ states that describe the occupation of the molecule cause all contributions to $\left\langle S_{x}\right\rangle$ and $\left\langle S_{y}\right\rangle$ to cancel. However, as in collinear system the $z$ components of the spin expectation values are not generally zero. In Fig. 6.22 we see that for high positive bias voltages the expectation value of the spin of the molecule is close to a half. As the angle is increased the expectation value gradually decreases to minus a half at $\phi=\pi$. A similar effect occurs for the electronic spin.

Also shown in Figs. 6.23, 6.24 and 6.25 are density plots of the corresponding offdiagonal reduced density matrix elements. Here we see that resonances in these plots occur along $\epsilon=-0.5 U$, where the detuning is zero, and very rapidly tend to zero either side of this line. The variation with the applied bias is related to the occupation probabilities of the $| \pm, \mp\rangle$ states. The resonances occur where overlap is greatest in the occupation probabilities of these states. The magnitude of the resonances are very small and therefore do not have a great effect on the current; they are greatest when $\phi=\frac{\pi}{2}$. At this angle the current is independent of the polarisation as both carriers have equal coupling strength to the spin up and spin down states in the molecule and as mentioned earlier, apart from the off-diagonal elements, the dynamics of the system 

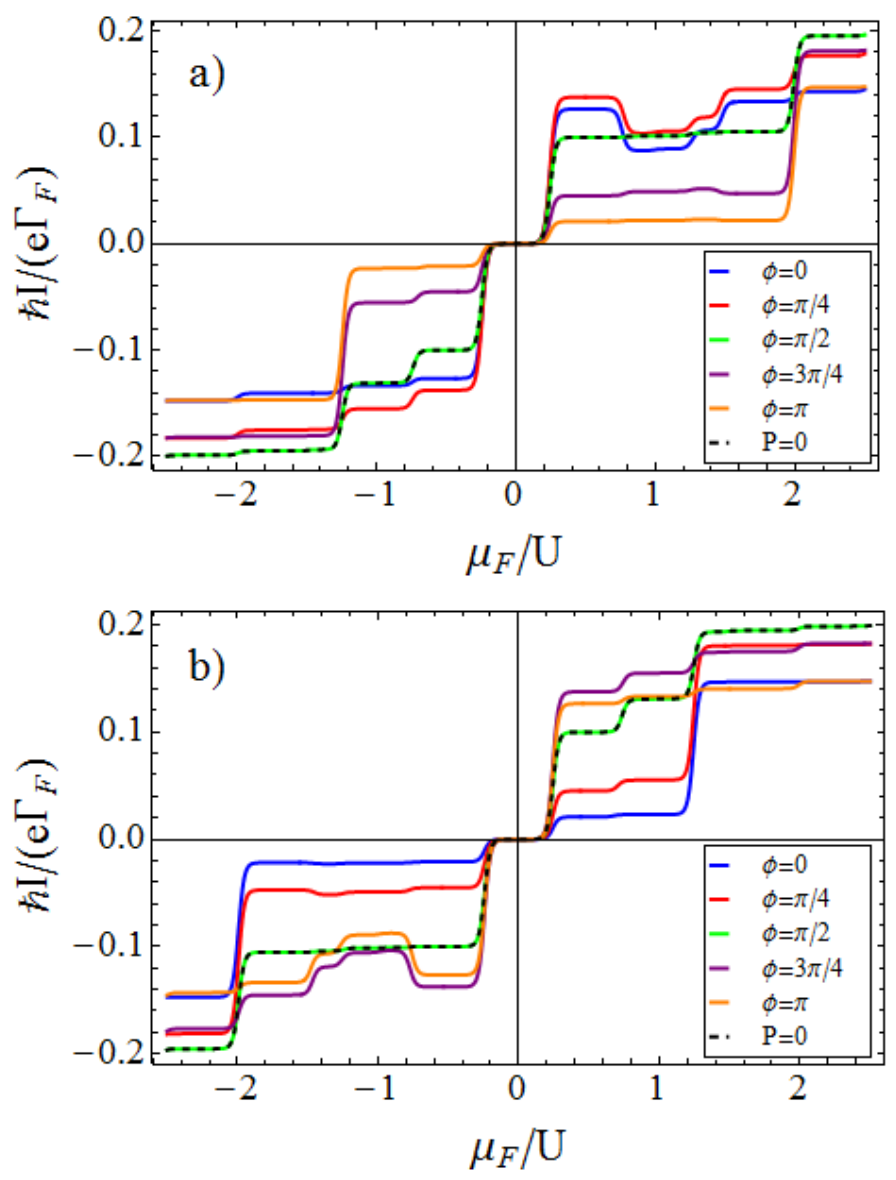

Figure 6.27: Plot of the current as a function of the chemical potential $\mu_{N}$ for various alignments of the magnetisation of the ferromagnetic lead with respect to the external magnetic field for (a) $\epsilon=0.5 \mathrm{U}$ and (b) $\epsilon=-1.5 \mathrm{U}$. The other parameters used in these plots are $J=0.5 U, B=1 U, \Gamma_{S}=1 U, P=0.8, k_{B} T=, 0.02 U$ and $\Gamma_{F}=0.001 U$.

are the same as those for the N-MM-S system. Therefore by comparing the current when $\phi=\frac{\pi}{2}$ to the current when $P=0$ we can see the effect of the off-diagonal elements. Figure 6.28 shows the difference in the current for these two cases. Here we see that the maximal current difference is only about $1 \%$ of the total current.

When $\epsilon=\frac{-U}{2}$ the superconducting proximity effect is in resonance and the current is maximal. With $\epsilon \neq \frac{-U}{2}$ we see a reduction in the maximum current at high bias and a different response to $\mu_{F}$ at intermediate values. In Fig. 6.27 we see that for some parameter values the current decreases with increasing $\mu_{F}$, an effect that does not occur when $\epsilon=\frac{-U}{2}$. These features are similar to the negative differential conductance features discussed in the previous section.

Figure 6.29 shows the differential conductance for various angles. $\phi$ increases from 


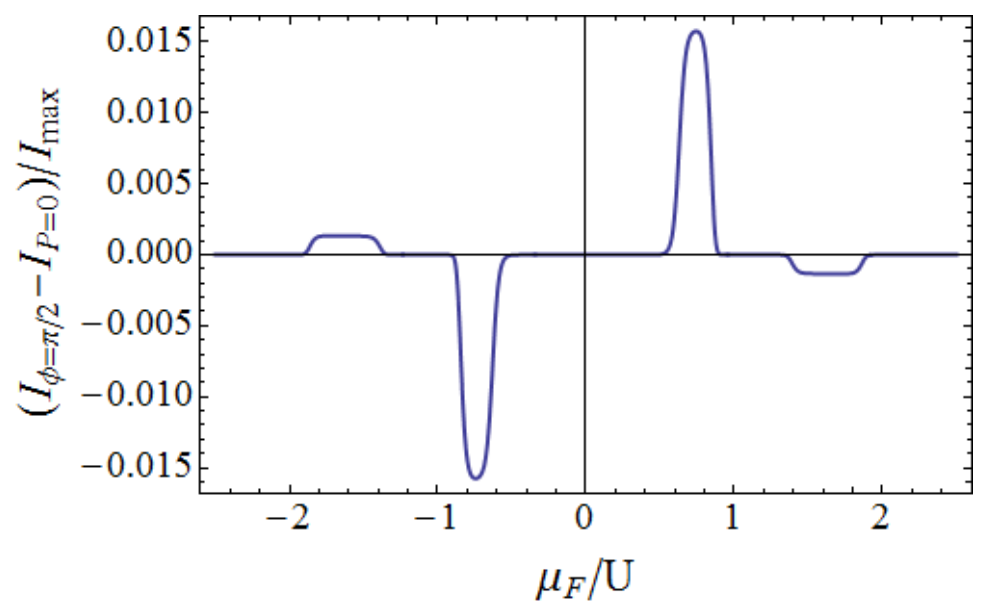

Figure 6.28: Plot of the difference in the current due to the off-diagonal elements. Plotted is $I(\phi=\pi / 2, P=0.8)-I(P=0)$ as a function of the chemical potential $\mu_{N}$. The other parameters used in this plot are $J=0.5 U, B=1 U, \Gamma_{S}=1 U, k_{B} T=0.02 U$ and $\Gamma_{F}=0.001 U$.

0 to $\pi / 2$ in plots a) to $d)$. This causes some features to become more prominent. Comparing plot d) to Fig. 6.13 we once again see that $I(\phi=\pi / 2) \approx I(P=0)$. All four plots show negative differential conductance features. These negative features only appear to occur near regions where excitation energies cross.

In the collinear case we found that the polarisation affected the maximum current and relative magnitude of the current for different bias voltages and level positions. We also found that when the polarisation is \pm 1 that the current is zero. With the magnetisation of the lead and the external magnetic field non-collinear the effects are similar. However the current is no longer zero when $P= \pm 1$. This can be understood by considering the transport sequence needed for current to flow in these systems. For current to flow into the superconducting lead, in the $\Delta \rightarrow \infty$ limit, the molecule needs to be doubly occupied. If we consider the situation where the molecule starts off unoccupied and the spin of the molecule is up, after some time a spin up electron tunnels from the metallic lead to the molecule. Transport on the molecule is now blocked for all spin up electrons in the ferromagnetic. After some time (that will depend on the polarisation of the lead) a spin down electron may tunnel on to the molecule. Once this happens transport from the molecule to the superconducting lead is possible. If the ferromagnetic lead is highly polarised then in the collinear case the current is limited by the tunneling of the minority carriers. If the magnetisation of the lead is not collinear with the quantisation axis of the molecule then there is a 

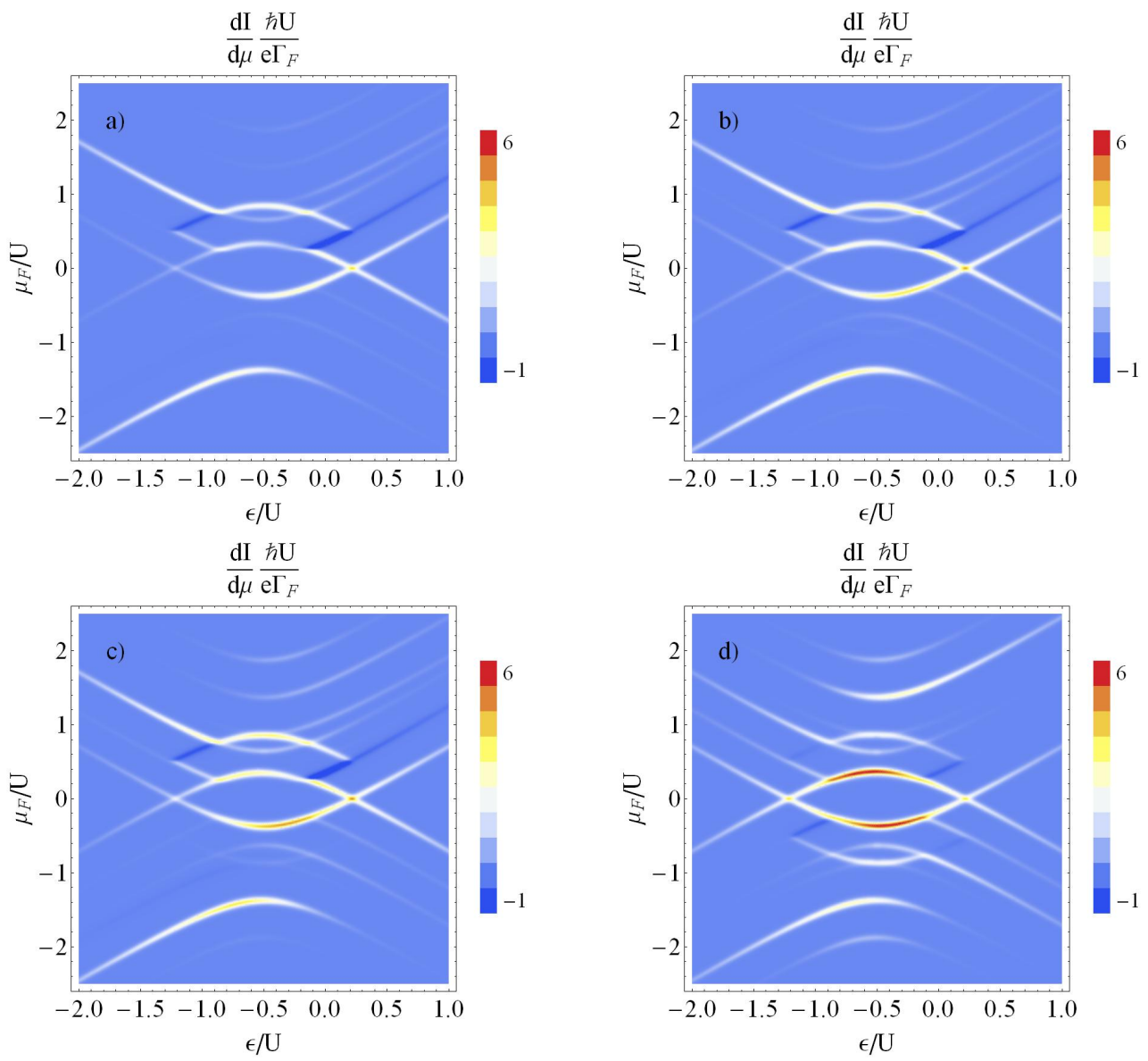

Figure 6.29: Density plots of the differential conductance as a function of the chemical potential $\mu_{N}$ and the level position $\epsilon$ for (a) $\phi=0$, (b) $\phi=\pi / 6$, (c) $\phi=\pi / 4$ and (d) $\phi=\pi / 2$. The other parameters used in these plots are $k_{B} T=0.02 U, J=0.5 U, B=1 U$, $\Gamma_{S}=1 U, P=0.8$ and $\Gamma_{F}=0.001 U$.

finite probability of both majority and minority carriers tunneling to the molecule and occupying it in the spin up or the spin down state, meaning there is a current even when there are only majority carriers.

For the collinear case we also found that the polarisation affects the current peak at high bias, resulting in the width at half maximum not being equal to $\Gamma_{S}$. In Fig. 6.30 we have plotted the normalised current at high bias, for various angles, as a function of $\epsilon$. Here we see that the angle affects the width of the current peak as well as the maximum current. As $\phi$ varies from $\pi / 2$ to 0 the current is suppressed, but comparatively less away from $\delta=0$. 


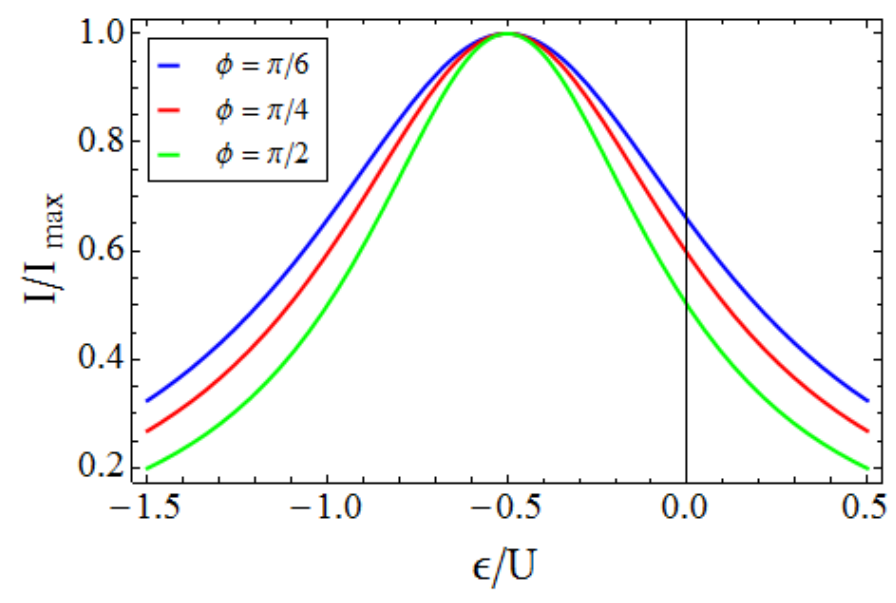

Figure 6.30: Plot of the normalised current, at high bias, as a function of the level position $\epsilon$. The parameters used in these plots are $\mu_{F}=3 U, J=0.5 U, B=1 U, \Gamma_{S}=1 U$, $P=0.8, k_{B} T=0.02 U$ and $\Gamma_{F}=0.001 U$.

\subsubsection{Varying $J, B$ and $\Gamma_{S}$, and the Effect of Shifting the Off Diagonal Reduced Density Matrix Element Resonances}

In Fig. 6.31 we show the current as a function of $\mu_{F}$ for various values of $J$. At $\mu_{F}=0$ there is a region where the current is zero; this region varies with $J$. In previous chapters the central zero current region increased in size as $J$ increased. This was because for the corresponding parameter values the singlet state is the ground state of the system and the energy of this state decreases with $J$. However here we see that the size of the zero current region first decreases and then increases as $J$ is increased. This is occurs because the ground state changes from $|I-\rangle$ to $|I S\rangle$. For $B>J$ the ground state is $|I-\rangle$. The steps in the current occur at the excitation energies and the width of these steps of course still dependents on the temperature. Figure 6.32 shows the current plotted as a function $\epsilon$ for various magnetic field strengths. Here we see similar effects as those observed for the exchange coupling. The value of $B$ affects the excitation energies, therefore the positions of the current steps are shifted as $B$ is varied. The maximum current is independent of magnetic field strength, but the voltage at which it is reached increases with $B$. The effect of varying $\Gamma_{S}$ is the same as described in Chapter 3. Increasing the coupling to the superconducting lead causing the superconducting proximity effect to be in resonance over a greater range of level positions, therefore the current is larger for $\delta \neq 0$. 


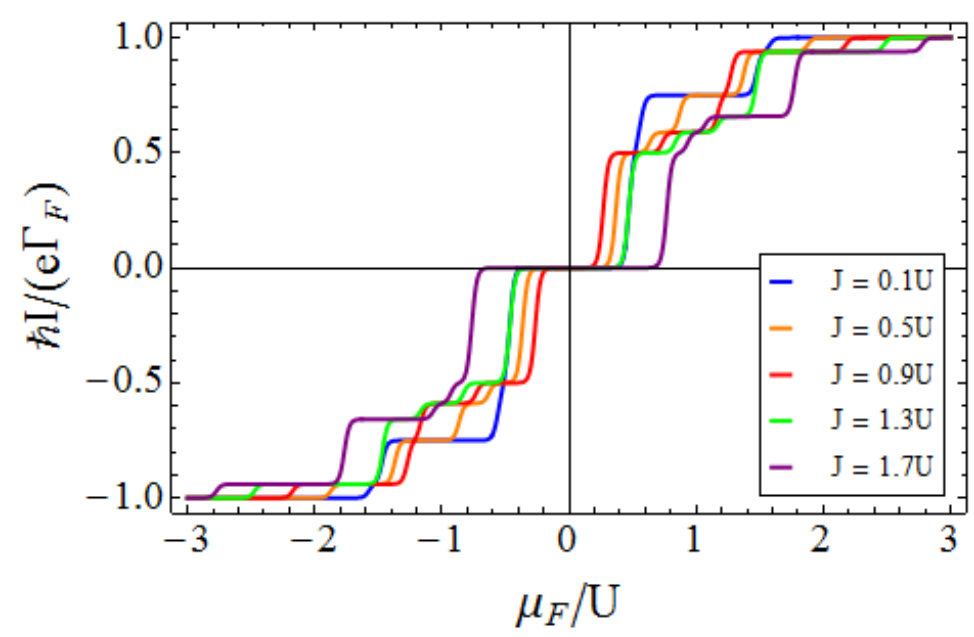

Figure 6.31: Plot of the current as a function of the chemical potential $\mu_{N}$ for various $J$ values. The parameters used in this plot are $\phi=\pi / 2, B=1 U, \Gamma_{S}=1 U, P=0.8$, $\epsilon=-0.5 U, k_{B} T=0.02 U$ and $\Gamma_{F}=0.001 U$.

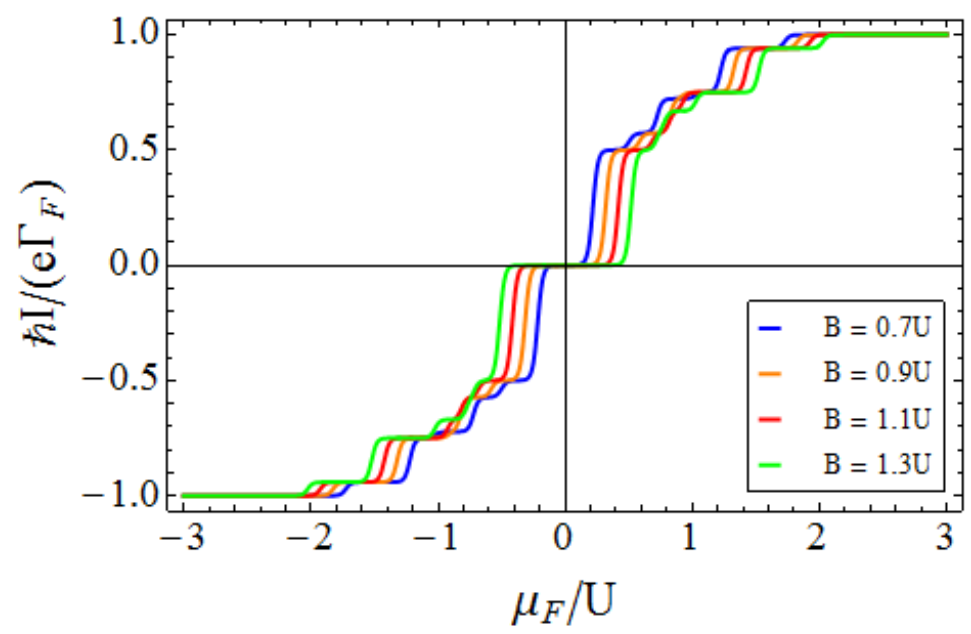

Figure 6.32: Plot of the current as a function of the chemical potential $\mu_{N}$ for various $B$ values. For the parameters used in these calculations there are no off-diagonal elements of the reduced density matrix that contribute to the current to first order in $\Gamma_{F}$. The parameters used in these plots are $\phi=\pi / 2, J=0.5 U, \Gamma_{S}=1.5 U, P=0.8, \epsilon=-0.5 U$, $k_{B} T=0.02 U$ and $\Gamma_{F}=0.001 U$.

When $B \neq \Gamma_{S}$ the resonances of the off-diagonal reduced density matrix elements are shifted away from $\delta=0$. This is shown in Fig. 6.33 for various $B$ and $\Gamma_{S}$ values. Comparing with plots d) of Figs. 6.23-6.25 we see that the magnitude of the resonances decrease as they are shifted away from $\delta=0$ and that they are spread over a greater 

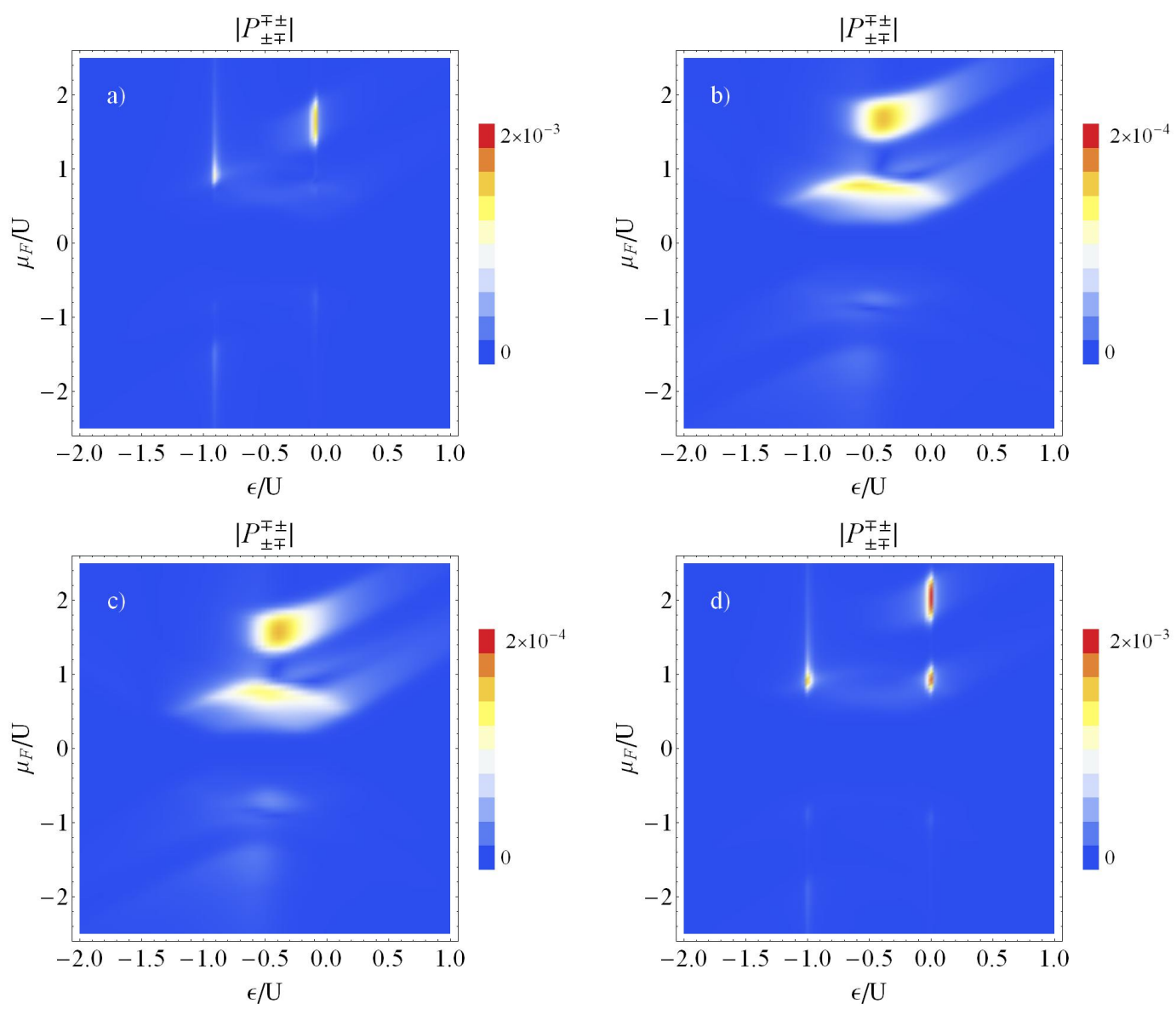

Figure 6.33: Density plots of the absolute value of the off-diagonal reduced density matrix elements as a function of the chemical potential $\mu_{N}$ and the level position $\epsilon$ for (a) $B=1 U, \Gamma_{S}=0.6 U$, (b) $B=1 U, \Gamma_{S}=1.1 U$, (c) $B=0.9 U, \Gamma_{S}=1 U$ and (d) $B=1.4 U, \Gamma_{S}=1 U$. The other parameters used in these plots are $\phi=\pi / 4, k_{B} T=0.05 U$, $J=0.5 U, P=0.8$ and $\Gamma_{F}=0.001 U$.

range of values. The resonance condition for the off-diagonal reduced density matrix elements is $\delta= \pm \sqrt{B^{2}-\Gamma_{S}^{2}}$. For the parameters of plots b) and c) the right side of this equation is complex and the condition cannot be achieved, thus we see that in these cases the off-diagonal elements are suppressed. The off-diagonal elements do not have a great effect on the current. This is shown in Fig. 6.34 where we have plotted $I(P=0.8, \phi=\pi / 2)-I(P=0)$, normalised by $I(P=0)$. The current is only changed by a fraction of a percent due to the off-diagonal density matrix elements. 


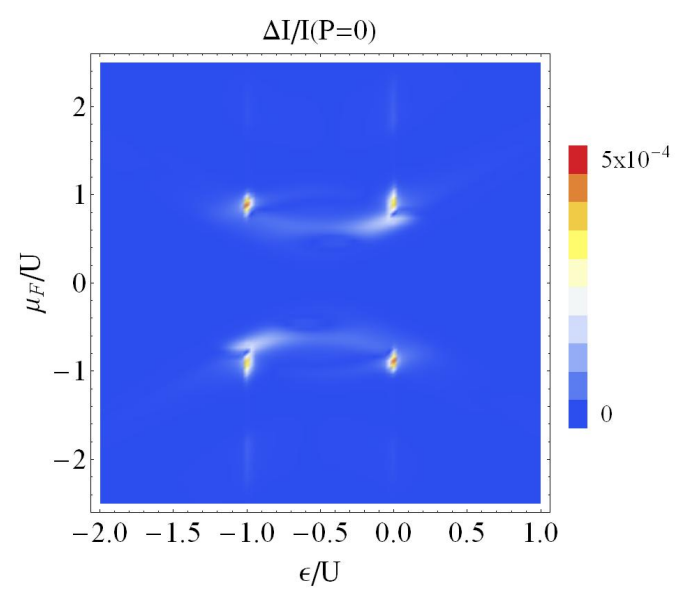

Figure 6.34: Density plot of $I(P=0.8, \phi=\pi / 2)-I(P=0)$, normalised by $I(P=0)$, as a function of the chemical potential $\mu_{N}$ and the level position $\epsilon$. The other parameters used in this plot are $k_{B} T=0.05 U, J=0.5 U, B=1.4 U, \Gamma_{S}=1 U, P=0.8$ and $\Gamma_{F}=0.001 U$.

\subsection{F-IMM-S Conclusions}

In this chapter we calculated the current through a system consisting of an isotropic magnetic molecule, in the presence of an external magnetic field, coupled to a ferromagnetic lead and an infinite gap superconducting lead. We separately considered the cases of the magnetisation of the ferromagnetic lead and the external magnetic field being collinear and non-collinear. In the latter case off-diagonal reduced density matrix elements contribute to the dynamics of the system. Generally many off-diagonal elements can contribute to the dynamics of the system in the non-collinear case, which can lead to a very large system of equations. To reduce the number of generalised transition rates to be calculated we worked in a regime where only $P_{-+}^{+-}$and $P_{+-}^{-+}$needed to be considered. To calculate the generalised transition rates involving the off-diagonal reduced density matrix elements we used the diagrammatic technique of Chapter 2.

As expected, in the collinear case we found that the maximum current is reduced when the polarisation of the ferromagnetic lead is non-zero. This is because the transport is restricted by the minority carriers. The occupation probabilities of the Andreev bound states also decrease when $P$ is non-zero. We can therefore conclude that the polarisation of the ferromagnetic lead suppresses the superconducting proximity effect. We also find that the spin expectation values are not generally zero when $P \neq 0$. This 
means that if the ferromagnetic lead is sufficiently polarised it should be possible to tune the spin of the molecule and the occupation of the molecule by adjusting the bias voltage and a gate voltage. Once again features in the current occur at the excitation energies, so it should also be possible to perform a spectroscopy of the excitation energies by measuring the current as functions of applied bias and gate voltages. The Fano factor calculations for this case show that the behaviour at high bias is not the same as for the N-IMM-S system. Here the Fano factor does not show Poissonian 2 particle transport when the superconducting proximity effect is off resonance, or Poissonian 1 particle transport on resonance. This is likely due to the suppression of superconducting proximity effect caused by the polarisation of the ferromagnetic lead.

In the non-collinear case we found that the current is greatest when the magnetisation of the ferromagnetic lead is perpendicular to the orientation of the external magnetic field. This is because under these circumstances the majority carriers can couple with equal strengths to the electronic spin up and spin down states of the molecule and thereby the current is not restricted by the minority carriers. The alignment of the magnetisation of the ferromagnetic lead also affects the superconducting proximity effect. For $|\phi| \neq \pi / 2$ the effect is suppressed or, as $\phi$ tends to $|\pi / 2|$, one could view this as the suppression due to $P$ being lifted. As for the collinear case we find that the spin expectation values can be non-zero. We also found that the off-diagonal reduced density matrix elements did not have a great affect on the dynamics of the system. Moreover, due to the specific form of the components of the $| \pm \mp\rangle$ states that correspond to the occupation of the molecule, the coherent superposition of these states does not lead to non-zero $x$ or $y$ molecular spin expectation values.

In both the collinear and non-collinear cases the external magnetic field caused the splitting of the previous degenerated orbitals. This leads to more excitation energies and therefore more differential conductance features. Because the external field lifts the degeneracy of the eigenstates, even when the second lead is not ferromagnetic the spin expectation values can be non-zero. Also observed for both cases is that when the polarisation is non-zero, or for certain external magnetic field strengths, negative differential conductances can occur. 


\section{Chapter 7}

\section{Summary and Conclusions}

In this thesis we have investigated electronic transport through a magnetic molecule coupled to an infinite gap superconducting lead and a second lead which is either a normal metallic lead, ferromagnetic or superconducting. To calculate the current through these systems we have used a real-time diagrammatic approach to perform a perturbation expansion in the tunneling coupling between the molecule and the leads. We were interested in the interplay between the superconducting proximity effect and the magnetism of the molecule, hence only the sub-gap transport between the molecule and the superconducting lead was relevant. We therefore worked in the $\Delta \rightarrow \infty$ limit. The Coulomb interaction on the molecule was taken into account exactly and the coupling to the superconducting lead was calculated to all orders and described by an effective Hamiltonian for the MM-S subsystem. In Chapter 3 we considered an isotropic molecule coupled to a superconducting lead and a normal metallic lead. In Chapter 4 we modified this system to allow for the molecule to be anisotropic. Then in Chapter 5 we investigated the Josephson current through the isotropic and the anisotropic molecules. Finally in Chapter 6 we analysed the dynamics of an isotropic molecule, in the presence of an external magnetic field, coupled to a superconducting lead and a ferromagnetic lead.

For each of the systems involving only one superconducting lead the current shows features that occur at the excitation energies of the eigenstates, and the current is maximal when the superconducting proximity effect is in resonance. The magnetisation of the molecule affects the current via the exchange coupling, the magnitude of which influences the size and number of steps in the current as a function of voltage. When the second lead is ferromagnetic then the proximity effect is suppressed, however this suppression is lifted when the magnetisation of the ferromagnetic lead is perpen- 
dicular to the magnetisation of the molecule, as under these conditions the current is not restricted by the minority carriers. The anisotropy, the quantum tunneling of magnetisation and the exchange coupling between the electronic and molecular spins all have no effect on the superconducting proximity effect. For the exchange coupling this is because the singly occupied states of the molecule do not couple to the superconducting lead. We also found that coupling to the ferromagnetic lead or the presence of an external magnetic field can cause the expectation values of the electronic and molecular spins to be non-zero. These effects indicate that it should be possible to use current measurements to probe the properties of a magnetic molecule and to use the bias voltage and a gate voltage to tune the occupation and spin of the molecule. The results for the Josephson current through both the isotropic and the anisotropic molecules show that a $0-\pi$ transition cannot occur in the infinite superconducting gap limit and that the anisotropy and QTM have negligible affect on the Josephson current.

We have studied in detail the transport dynamics of the aforementioned systems in the infinite superconducting gap limit. It would be interesting to extend this work to the finite $\Delta$ limit. For the Josephson current this already been done in the infinite Coulomb interaction limit $[20,21]$, however for the other systems no such studies have been carried out. To treat a finite Coulomb interaction exactly and to allow the superconducting gap to be finite is a very computationally expensive task. In contrast to the $\Delta \rightarrow \infty$ limit it would not be practically possible to treat the coupling to the superconducting lead exactly. For the F-IMM-S system we restricted ourselves to a regime where only the $P_{ \pm \mp}^{\mp \pm}$ off-diagonal elements needed to be considered. Working in a different regime where coherent superpositions of states with either the same molecular spin, or the same occupation of the molecule, contribute to the dynamics of the system could lead to interesting results, as such superpositions could cause spin precession or spin accumulation.

The results are indicative of the transport properties that may be observed in future experiments involving magnetic molecules coupled to superconducting and nonsuperconducting metallic leads. Ideally these results will aid with the interpretation of current measurements and characterisation of the magnetic properties of the molecules in question. 


\section{Appendix A}

\section{Diagrammatic Rules}

Here we give the diagrammatic rules for perturbation expansions in both $\Gamma_{S}$ and $\Gamma_{N}$. These rules can be used to calculate generalised transition rates to any order in tunnel coupling. These rules are a modified version of those presented in Ref. [5].

1. Draw all topologically different diagrams with fixed ordering of the vertices and tunneling lines connecting the vertices in pairs. Assign the energy $\omega_{i}$ o each tunneling line and assign energies to all propagators according to the state of the molecule. Tunneling lines connecting two lead creation (or annihilation) operators shall be called incoming anomalous lines (outgoing anomalous lines) and should be drawn with two arrows pointing away from (towards) each other. For each anomalous line choose the direction with respect to the Keldysh contour (forward or backward) arbitrarily.

2. For each part of the diagram between adjacent vertices (on the top or bottom propagator) assign a factor $\frac{1}{\Delta E+i 0^{+}}$, where $\Delta E$ is the energy difference between left-going and right-going propagators and tunneling lines.

3. For each tunneling line the diagram acquires a factor of $\frac{1}{2 \pi} \Gamma_{\eta} D_{\eta}\left(\omega_{i}\right) f_{\eta}^{\alpha}\left(\omega_{i}\right)$, where $D_{\eta}(\omega)=\frac{\left|\omega-\mu_{\eta}\right|}{\sqrt{\left(\omega-\mu_{\eta}\right)^{2}-\left|\Delta_{\eta}\right|^{2}}} \theta\left(\left|\omega-\mu_{\eta}\right|-\left|\Delta_{\eta}\right|\right)$. For lines going backward (forward) with respect to the Keldysh contour $\alpha=+(-)$. For anomalous lines also multiply by a factor $\alpha \operatorname{sign}\left(\omega_{i}-\mu_{\eta}\right) \frac{\left|\Delta_{\eta}\right|}{\left|\omega_{i}-\mu_{\eta}\right|} e^{ \pm i \phi_{\eta}}$, where $+(-)$ is for incoming (outgoing) anomalous lines. For normal leads no anomalous lines appear and $D_{\eta}\left(\omega_{i}\right)=1$.

4. Multiply by an overall prefactor $-i$ and assign an additional factor -1 for each (a) vertex on the lower propagator; (b) crossing of tunneling lines; (c) vertex that connects a doubly occupied state of the molecule to a spin up state; (d) outgoing 
(incoming) anomalous tunneling line for which the vertex that appears earlier (later) on the Keldysh contour involves a spin up electron of the molecule.

5. Integrate the diagrams over all energies $\omega_{i}$, then sum over all diagrams. 


\section{Appendix B}

\section{Coefficients of the Eigenstates of the AMM-S System}

Here we give the eigenstates and energies of the AMM-S subsystem studied in chapter 4. The 12 eigenstates are

$$
\begin{gathered}
|A\rangle=X 1_{A}|0,0\rangle+X 2_{A}|d, 0\rangle, \\
|B\rangle=X 1_{B}|0,-1\rangle+X 2_{B}|d,-1\rangle+X 3_{B}|0,1\rangle+X 4_{B}|d, 1\rangle, \\
|C\rangle=X 1_{C}|\uparrow, 0\rangle+X 2_{C}|\downarrow, 1\rangle+X 3_{C}|\downarrow,-1\rangle
\end{gathered}
$$

and

$$
|D\rangle=X 1_{D}|\downarrow, 0\rangle+X 2_{D}|\uparrow,-1\rangle+X 3_{D}|\uparrow, 1\rangle,
$$

where $A \rightarrow A 1, A 2, B \rightarrow B 1, B 2, B 3, B 4, C \rightarrow C 1, C 2, C 3$ and $D \rightarrow D 1, D 2, D 3$. The corresponding energies are given in Table B.1. To give the expression for the coefficients of the components of the eigenstates we first define some parameters;

$$
\begin{aligned}
& Z_{--}=\sqrt{8+\frac{4(\rho+\delta)^{2}}{\Gamma_{s}^{2}}+\frac{\left(-4 M^{2}+\Gamma_{s}^{2}+(2 M+\rho+\delta)^{2}\right)^{2}}{(2 M+\rho)^{2} \Gamma_{s}^{2}}}, \\
& Z_{+-}=\sqrt{8+\frac{4(\rho+\delta)^{2}}{\Gamma_{s}^{2}}+\frac{\left(-4 M^{2}+\Gamma_{s}^{2}+(-2 M+\rho+\delta)^{2}\right)^{2}}{(-2 M+\rho)^{2} \Gamma_{s}^{2}}}, \\
& Z_{-+}=\sqrt{8+\frac{4(\rho-\delta)^{2}}{\Gamma_{s}^{2}}+\frac{\left(-4 M^{2}+\Gamma_{s}^{2}+(2 M-\rho+\delta)^{2}\right)^{2}}{(-2 M+\rho)^{2} \Gamma_{s}^{2}}}
\end{aligned}
$$




$$
\begin{aligned}
Z_{++} & =\sqrt{8+\frac{4(\rho-\delta)^{2}}{\Gamma_{s}^{2}}+\frac{\left(-4 M^{2}+\Gamma_{s}^{2}+(2 M+\rho-\delta)^{2}\right)^{2}}{(2 M+\rho)^{2} \Gamma_{s}^{2}}}, \\
Z_{1} & =\sqrt{4+2\left(\frac{\xi_{C 1}}{M J}\right)^{2}+\left(\frac{2 D+2 E_{C 1}-J-2 \epsilon}{M}\right)^{2}} \\
Z_{2} & =\sqrt{4+2\left(\frac{\xi_{C 2}}{M J}\right)^{2}+\left(\frac{2 D+2 E_{C 2}-J-2 \epsilon}{M}\right)^{2}} \\
Z_{3} & =\sqrt{4+2\left(\frac{\xi_{C 3}}{M J}\right)^{2}+\left(\frac{2 D+2 E_{C 3}-J-2 \epsilon}{M}\right)^{2}}
\end{aligned}
$$

with

$$
\rho=\sqrt{U^{2}+\Gamma_{s}^{2}+4 U \epsilon+4 \epsilon^{2}}
$$

and

$$
\xi_{C}=2 M^{2}-1 / 2\left(2 D+2 E_{C}-J-2 \epsilon\right)\left(2 D+2 E_{C}+J-2 \epsilon\right) .
$$

The coefficients of the eigenstates are given in Tables B.2, B.3, B.4 and B.5.

\begin{tabular}{|c||c|c|c|}
\hline$\chi$ & $A$ & $B$ & $C / D$ \\
\hline \hline$E_{\chi_{1}}$ & $\frac{\delta}{2}+\epsilon_{A}$ & $\frac{\delta}{2}-\epsilon_{A}-M-D$ & $\operatorname{Re}\left[\frac{\gamma+\beta^{2}+i \sqrt{3\left(\gamma-\beta^{2}\right)^{2}}}{12 \beta}\right]+\frac{3 \epsilon-2 D}{3}$ \\
$E_{\chi_{2}}$ & $\frac{\delta}{2}-\epsilon_{A}$ & $\frac{\delta}{2}-\epsilon_{A}+M-D$ & $\operatorname{Re}\left[\frac{\gamma+\beta^{2}-i \sqrt{3\left(\beta^{2}-\gamma\right)^{2}}}{12 \beta}\right]+\frac{3 \epsilon-2 D}{3}$ \\
$E_{\chi_{3}}$ & - & $\frac{\delta}{2}+\epsilon_{A}-M-D$ & $\operatorname{Re}\left[-\frac{\gamma+\beta^{2}}{6 \beta}\right]+\frac{3 \epsilon-2 D}{3}$ \\
$E_{\chi_{4}}$ & - & $\frac{\delta}{2}+\epsilon_{A}+M-D$ & - \\
\hline
\end{tabular}

Table B.1: Energies of the 12 eigenstates of the anisotropic system. $\delta$ and $\epsilon_{A}$ are defined as in Chapters 3 and 6 . The other terms are defined as $\beta=\left(\sqrt{\alpha}+72 M^{2} D-8 D^{3}+\right.$ $\left.27 J^{3}\right)^{1 / 3}, \gamma=12 M^{2}+4 D^{2}+9 J^{2}$ and $\alpha=-\left(12 M^{2}+4 D^{2}+9 J^{2}\right)^{3}+\left(72 M^{2} D-8 D^{3}+27 J^{3}\right)^{2}$.

\begin{tabular}{|c||c|c|}
\hline$\chi$ & $A 1$ & $A 2$ \\
\hline \hline$X 1_{\chi}$ & $\frac{1}{\sqrt{2}} \sqrt{1-\frac{\delta}{2 \epsilon_{A}}}$ & $\frac{1}{\sqrt{2}} \sqrt{1+\frac{\delta}{2 \epsilon_{A}}}$ \\
$X 2_{\chi}$ & $\frac{-1}{\sqrt{2}} \sqrt{1+\frac{\delta}{2 \epsilon_{A}}}$ & $\frac{1}{\sqrt{2}} \sqrt{1-\frac{\delta}{2 \epsilon_{A}}}$ \\
\hline
\end{tabular}

Table B.2: Coefficients of the $|A\rangle$ eigenstates. 


\begin{tabular}{|c||c|c|c|c|}
\hline$\chi$ & $B 1$ & $B 2$ & $B 3$ & $B 4$ \\
\hline \hline$X 1_{\chi}$ & $\frac{2(\rho+\delta)}{Z_{--} \Gamma_{s}}$ & $-\frac{2(\rho+\delta)}{Z_{+-} \Gamma_{s}}$ & $-\frac{2(\rho-\delta)}{Z_{-+} \Gamma_{s}}$ & $\frac{2(\rho-\delta)}{Z_{++} \Gamma_{s}}$ \\
$X 2_{\chi}$ & $\frac{2}{Z_{--}}$ & $-\frac{2}{Z_{+-}}$ & $\frac{2}{Z_{-+}}$ & $-\frac{2}{Z_{++}}$ \\
$X 3_{\chi}$ & $\frac{2(\rho+\delta)}{Z_{--} \Gamma_{s}}$ & $\frac{2(\rho+\delta)}{Z_{+-} \Gamma_{s}}$ & $-\frac{2(\rho-\delta)}{Z_{-+} \Gamma_{s}}$ & $-\frac{2(\rho-\delta)}{Z_{++} \Gamma_{s}}$ \\
$X 4_{\chi}$ & $\frac{2}{Z_{--}}$ & $\frac{2}{Z_{+-}}$ & $\frac{2}{Z_{-+}}$ & $\frac{2}{Z_{++}}$ \\
\hline
\end{tabular}

Table B.3: Coefficients of the $|B\rangle$ eigenstates. 


\begin{tabular}{|c||c|c|c|}
\hline$\chi$ & $C 1$ & $C 2$ & $C 3$ \\
\hline \hline$X 1_{\chi}$ & $\frac{4\left(M+D+E_{C 1}-\epsilon\right)\left(M-D-E_{C 1}+\epsilon\right)+J^{2}}{M J \sqrt{2} Z_{1}}$ & $\frac{4\left(M+D+E_{C 2}-\epsilon\right)\left(M-D-E_{C 2}+\epsilon\right)+J^{2}}{M J \sqrt{2} Z_{2}}$ & $\frac{4\left(M+D+E_{C 3}-\epsilon\right)\left(M-D-E_{C 3}+\epsilon\right)+J^{2}}{M J \sqrt{2} Z_{3}}$ \\
$X 2_{\chi}$ & $\frac{-2 D-2 E_{C 1}+J+2 \epsilon}{M Z_{1}}$ & $\frac{-2 D-2 E_{C 2}+J+2 \epsilon}{M Z_{2}}$ & $\frac{-2 D-2 E_{C 3}+J+2 \epsilon}{M Z_{3}}$ \\
$X 3_{\chi}$ & $\frac{2}{Z_{1}}$ & $\frac{2}{Z_{2}}$ & $\frac{2}{Z_{3}}$ \\
\hline
\end{tabular}

Table B.4: Coefficients of the $|C\rangle$ eigenstates.

\begin{tabular}{|c||c|c|c|}
\hline$\chi$ & $D 1$ & $D 2$ & $D 3$ \\
\hline \hline$X 1_{\chi}$ & $\frac{4\left(M+D+E_{D 1}-\epsilon\right)\left(M-D-E_{D 1}+\epsilon\right)+J^{2}}{M J \sqrt{2} C r 1}$ & $\frac{4\left(M+D+E_{D 2}-\epsilon\right)\left(M-D-E_{D 2}+\epsilon\right)+J^{2}}{M J \sqrt{2} C r 2}$ & $\frac{4\left(M+D+E_{D 3}-\epsilon\right)\left(M-D-E_{D 3}+\epsilon\right)+J^{2}}{M J \sqrt{2} C r 3}$ \\
$X 2_{\chi}$ & $\frac{-2 D-2 E_{D 1}+J+2 \epsilon}{M C r 1}$ & $\frac{-2 D-2 E_{D 2}+J+2 \epsilon}{M C r 2}$ & $\frac{-2 D-2 E_{D 3}+J+2 \epsilon}{M C r 3}$ \\
$X 3_{\chi}$ & $\frac{2}{C r 1}$ & $\frac{2}{C r 2}$ & $\frac{2}{C r 3}$ \\
\hline
\end{tabular}

Table B.5: Coefficients of the $|D\rangle$ eigenstates. 


\section{Appendix C}

\section{Generalised Transition Rates of the F-IMM-S System}

\section{C.1 Solving Integrals of Generalised Transition Rates}

To solve the integrals that arise when calculating generalised transition rates that involve off-diagonal reduced density matrix elements we use the residue theorem. This theorem states

$$
\oint_{\gamma} f(z) d z=2 \pi i \sum_{k=1}^{N} \operatorname{Res}_{z=z_{k}} f(z)
$$

where $f(z)$ is a single-valued analytic function everywhere on $\gamma$ and inside $\gamma$ except at a finite number of singular points $z_{1}, z_{2} \ldots z_{N}$, and the contour $\gamma$ is positively oriented. The residual of $f(z)$ at $z=a$ is given by

$$
\operatorname{Res}_{z=a} f(z)=\lim _{z \rightarrow a}[(z-a) f(z)]
$$

The residue theorem can be used to calculate an integral of the form $\int_{-\infty}^{\infty} f(z) d z$ if certain conditions are met. Figure C.1 shows two contour integrals with poles contained in the contours. If $|z| \rightarrow \infty$ and the contributions to the integrals from the upper and lower contours cancel for $\alpha \neq 0, \pi$, then the integral from $-\infty$ to $\infty$ can be calculated using the residuals of the poles contained in the contours. Writing this out we get

$$
\oint_{\gamma^{+}} f(z) d z+\oint_{\gamma^{-}} f(z) d z=2 \pi i\left(\sum_{k=1}^{N^{+}} \operatorname{Res}_{z^{+}=z_{k}^{+}} f(z)-\sum_{k=1}^{N^{-}} \operatorname{Res}_{z^{-}=z_{k}^{-}} f(z)\right)
$$


where $\gamma^{+}$and $\gamma^{-}$indicate the upper and lower contours, respectively, and the contribution from lower contour is negative due to the clockwise orientation of the contour. Then, for $|z| \rightarrow \infty$ and contributions for complex $z$ values canceling, this equation becomes

$$
2 \int_{-\infty}^{\infty} f(z) d z=2 \pi i \sum_{k=1}^{N} \operatorname{Res}_{z=z_{k}} f(z)
$$

where the sum is now over all poles and those from the lower half plane should be multiplied by a factor of -1 . The integrals we are concerned with meet the appropriate criteria to be calculated in this manor.

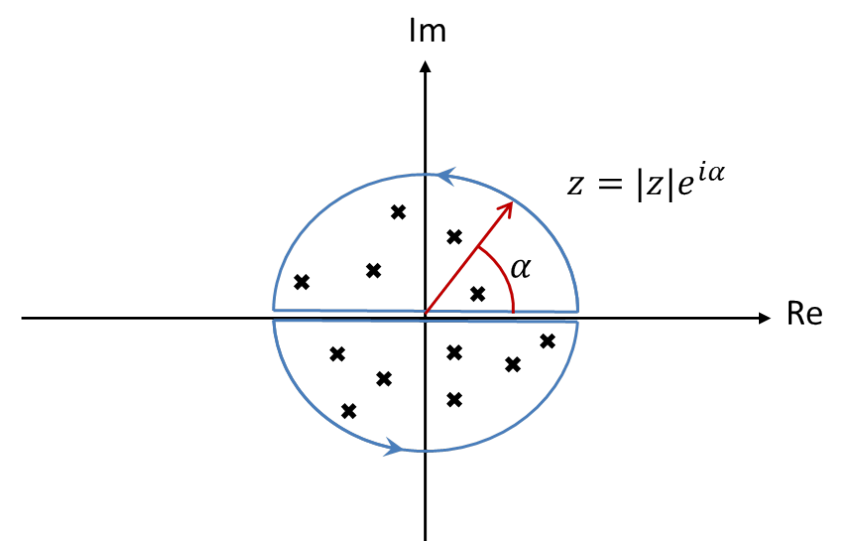

Figure C.1: Two contour integrals of a function, one in the upper half plane and the other in the lower half plane. The crosses indicate poles of the function in question.

We will now use the above equations to solve the integral

$$
\int_{-\infty}^{\infty} g(\omega) d \omega=-\int_{-\infty}^{\infty} f^{+}(\omega)\left(\frac{\epsilon_{1}-\epsilon_{2}+2 i 0^{+}}{\left(\epsilon_{1}-\omega+i 0^{+}\right)\left(\omega-\epsilon_{2}+i 0^{+}\right)}\right) d \omega
$$

where $f^{+}(\omega)=\frac{1}{\mathrm{e}^{\beta(\omega-\mu)}+1}$ is the Fermi function. The integrand has poles in the upper half of the complex plane at

$$
\omega=\epsilon_{1}+i 0^{+}
$$

and

$$
\omega=\omega_{n}^{+}=\frac{2 \pi i}{\beta}\left(n+\frac{1}{2}\right)+\mu .
$$

In the lower half plane it has poles at

$$
\omega=\epsilon_{2}-i 0^{+}
$$


and

$$
\omega=\omega_{n}^{-}=-\frac{2 \pi i}{\beta}\left(n+\frac{1}{2}\right)+\mu .
$$

Here $n \in \mathbb{N}^{0}$ and $\beta=1 / k_{B} T$. The poles at $\pm \omega_{n}$ are the poles of the Fermi function. Using Eq. C.4 the integral is given by

$$
\int_{-\infty}^{\infty} g(\omega) d \omega=-\frac{2 \pi i}{2} \sum_{n=1}^{N} \operatorname{Res}_{z=z_{n}}\left[f^{+}(\omega)\left(\frac{\epsilon_{1}-\epsilon_{2}+2 i 0^{+}}{\left(\epsilon_{1}-\omega+i 0^{+}\right)\left(\omega-\epsilon_{2}+i 0^{+}\right)}\right)\right] .
$$

Substituting in the residuals at the various poles we get

$$
\begin{array}{r}
\int_{-\infty}^{\infty} g(\omega) d \omega=-\pi i\left(\epsilon_{1}-\epsilon_{2}+2 i 0^{+}\right)\left[\frac{f^{+}\left(\epsilon_{1}+i 0^{+}\right)}{\epsilon_{1}-\epsilon_{2}+2 i 0^{+}}-\frac{f^{+}\left(\epsilon_{2}-i 0^{+}\right)}{\epsilon_{2}-\epsilon_{1}-2 i 0^{+}}\right. \\
\left.-\sum_{n=0}^{\infty}\left(\frac{1}{\beta\left(\omega_{n}^{+}-\epsilon_{1}-i 0^{+}\right)\left(\omega_{n}^{+}-\epsilon_{2}+i 0^{+}\right)}-\frac{1}{\beta\left(\omega_{n}^{-}-\epsilon_{1}-i 0^{+}\right)\left(\omega_{n}^{-}-\epsilon_{2}+i 0^{+}\right)}\right)\right]
\end{array}
$$

where terms that arise from poles in the negative half plane are multiplied by an additional factor of -1 . We can now let $0^{+}$tend to zero to give

$$
\int_{-\infty}^{\infty} g(\omega) d \omega=-\pi i\left[f^{+}\left(\epsilon_{1}\right)+f^{+}\left(\epsilon_{2}\right)-\frac{\epsilon_{1}-\epsilon_{2}}{\beta} \sum_{n=0}^{\infty}\left(\frac{1}{\left(\omega_{n}^{+}-\epsilon_{1}\right)\left(\omega_{n}^{+}-\epsilon_{2}\right)}-\frac{1}{\left(\omega_{n}^{-}-\epsilon_{1}\right)\left(\omega_{n}^{-}-\epsilon_{2}\right)}\right)\right] .
$$

Rewriting the term in the sum we get

$$
\int_{-\infty}^{\infty} g(\omega) d \omega=-\pi i\left[f^{+}\left(\epsilon_{1}\right)+f^{+}\left(\epsilon_{2}\right)-\frac{1}{\beta} \sum_{n=0}^{\infty}\left(\frac{1}{\left(\omega_{n}^{+}-\epsilon_{1}\right)}-\frac{1}{\left(\omega_{n}^{+}-\epsilon_{2}\right)}-\frac{1}{\left(\omega_{n}^{-}-\epsilon_{1}\right)}+\frac{1}{\left(\omega_{n}^{-}-\epsilon_{2}\right)}\right)\right] .
$$

We now substitute in the expressions for $\omega_{n}^{ \pm}$and use properties of the digamma function $\Psi(x)$,

$$
\Psi(x)-\Psi(y)=\sum_{n=0}^{\infty}\left(\frac{-1}{x+n}+\frac{1}{y+n}\right)
$$

and

$$
\Psi\left(x^{*}\right)=\Psi^{*}(x)
$$

to express the sum terms. The integral is then given by

$$
\int_{-\infty}^{\infty} g(\omega) d \omega=-\pi i\left[f^{+}\left(\epsilon_{1}\right)+f^{+}\left(\epsilon_{2}\right)\right]-\operatorname{Re}\left[\Psi\left(\frac{1}{2}+\frac{i \beta}{2 \pi}\left(\epsilon_{1}-\mu\right)\right)-\Psi\left(\frac{1}{2}+\frac{i \beta}{2 \pi}\left(\epsilon_{2}-\mu\right)\right)\right] .
$$




\section{C.2 Generalised Transition Rates}

The elements of the kernel $\mathbf{W}$ are given by;

$$
\begin{aligned}
& W_{\eta+ \pm}=\frac{\Gamma_{F}}{4}\left[\delta_{+}^{2}(1 \pm P \cos \phi) f^{-}\left(E_{+ \pm}-E_{\eta}\right)+\delta_{-}^{2}(1 \mp P \cos \phi) f^{+}\left(E-\eta-E_{+ \pm}\right)\right], \\
& W_{\eta- \pm}=\frac{\Gamma_{F}}{4}\left[\delta_{-}^{2}(1 \pm P \cos \phi) f^{-}\left(E_{- \pm}-E_{\eta}\right)+\delta_{+}^{2}(1 \mp P \cos \phi) f^{+}\left(E-\eta-E_{- \pm}\right)\right], \\
& W_{I+ \pm+}=\frac{\Gamma_{F}}{2}\left[\delta_{ \pm}^{2}(1-P \cos \phi) f^{-}\left(E_{ \pm+}-E_{I+}\right)+\delta_{\mp}^{2}(1+P \cos \phi) f^{+}\left(E_{I+}-E_{ \pm+}\right)\right], \\
& W_{I- \pm-}=\frac{\Gamma_{F}}{2}\left[\delta_{ \pm}^{2}(1+P \cos \phi) f^{-}\left(E_{ \pm-}-E_{I-}\right)+\delta_{\mp}^{2}(1-P \cos \phi) f^{+}\left(E_{I-}-E_{ \pm-}\right)\right],
\end{aligned}
$$

with the rates for the reverse transitions (eg. $W_{+ \pm \eta}$ ) are obtained by switching the signs of the Fermi functions, and

$$
\begin{aligned}
& W_{\mp \pm \eta}^{ \pm \mp \eta}=\alpha \frac{i \delta_{+} \delta_{-}}{8 \pi} \Gamma_{F} P \sin \phi\left(i \pi \left(f^{+}\left(E_{+-}-E_{\eta}\right)+f^{+}\left(E_{-+}-E_{\eta}\right)+f^{-}\left(E_{\eta}-E_{-+}\right)+\right.\right. \\
&\left.f^{-}\left(E_{\eta}-E_{+-}\right)\right) \mp \operatorname{Re}\left[\Psi\left(\frac{1}{2}+\frac{i \beta}{2 \pi}\left(E_{\eta}-E_{+-}-\mu_{F}\right)\right)-\Psi\left(\frac{1}{2}+\frac{i \beta}{2 \pi}\left(E_{\eta}-E_{-+}-\mu_{F}\right)\right)\right] \\
&\left.\mp \operatorname{Re}\left[\Psi\left(\frac{1}{2}+\frac{i \beta}{2 \pi}\left(E_{+-}-E_{\eta}-\mu_{F}\right)\right)-\Psi\left(\frac{1}{2}+\frac{i \beta}{2 \pi}\left(E_{-+}-E_{\eta}-\mu_{F}\right)\right)\right]\right) \quad(\text { C. } 21)
\end{aligned}
$$

and

$$
\begin{gathered}
W_{\eta \mp \pm}^{\eta \pm \mp}=\alpha \frac{i \delta_{+} \delta_{-}}{8 \pi} \Gamma_{F} P \sin \phi\left(i \pi \left(f^{+}\left(E_{\eta}-E_{-+}\right)+f^{+}\left(E_{\eta}-E_{+-}\right)+f^{-}\left(E_{+-}-E_{\eta}\right)+\right.\right. \\
\left.f^{-}\left(E_{-+}-E_{\eta}\right)\right) \mp \operatorname{Re}\left[\Psi\left(\frac{1}{2}+\frac{i \beta}{2 \pi}\left(E_{-+}-E_{\eta}-\mu_{F}\right)\right)-\Psi\left(\frac{1}{2}+\frac{i \beta}{2 \pi}\left(E_{+-}-E_{\eta}-\mu_{F}\right)\right)\right] \\
\left.\mp \operatorname{Re}\left[\Psi\left(\frac{1}{2}+\frac{i \beta}{2 \pi}\left(E_{\eta}-E_{-+}-\mu_{F}\right)\right)-\Psi\left(\frac{1}{2}+\frac{i \beta}{2 \pi}\left(E_{\eta}-E_{+-}-\mu_{F}\right)\right)\right]\right), \quad(\text { C. } 22)
\end{gathered}
$$

where $\eta=I 0, I S$ and $\alpha=+(-)$ if $\eta=I 0(I S)$, and

$$
\begin{gathered}
W_{ \pm+ \pm+}=\frac{-\Gamma_{F}}{2}\left[( 1 + P \operatorname { c o s } \phi ) \left(\delta_{\mp}^{2} f^{+}\left(E_{I+}-E_{ \pm+}\right)+\frac{\delta_{ \pm}^{2}}{2}\left[f^{-}\left(E_{ \pm+}-E_{I 0}\right)\right.\right.\right. \\
\left.\left.+f^{-}\left(E_{ \pm+}-E_{I S}\right)\right]\right)+(1-P \cos \phi)\left(\delta_{ \pm}^{2} f^{-}\left(E_{ \pm+}-E_{I+}\right)\right. \\
\left.\left.+\frac{\delta_{\mp}^{2}}{2}\left[f^{+}\left(E_{I 0}-E_{ \pm+}\right)+f^{+}\left(E_{I S}-E_{ \pm+}\right)\right]\right)\right] \\
W_{ \pm+ \pm+}=\frac{-\Gamma_{F}}{2}\left[( 1 - P \operatorname { c o s } \phi ) \left(\delta_{\mp}^{2} f^{+}\left(E_{I-}-E_{ \pm-}\right)+\frac{\delta_{ \pm}^{2}}{2}\left[f^{-}\left(E_{ \pm-}-E_{I 0}\right)\right.\right.\right.
\end{gathered}
$$




$$
\begin{gathered}
\left.\left.+f^{-}\left(E_{ \pm-}-E_{I S}\right)\right]\right)+(1+P \cos \phi)\left(\delta_{ \pm}^{2} f^{-}\left(E_{ \pm-}-E_{I-}\right)\right. \\
\left.\left.+\frac{\delta_{\mp}^{2}}{2}\left[f^{+}\left(E_{I 0}-E_{ \pm-}\right)+f^{+}\left(E_{I S}-E_{ \pm-}\right)\right]\right)\right] \\
W_{I \pm I \pm}=\frac{-\Gamma_{F}}{2}\left[(1 \pm P \cos \phi)\left(\delta_{-}^{2} f^{-}\left(E_{I \pm}-E_{+ \pm}\right)+\delta_{+}^{2} f^{-}\left(E_{I \pm}-E_{- \pm}\right)\right)\right. \\
\left.+(1 \mp P \cos \phi)\left(\delta_{+}^{2} f^{+}\left(E_{+ \pm}-E_{I \pm}\right)+\delta_{-}^{2} f^{+}\left(E_{- \pm}-E_{I \pm}\right)\right)\right] \\
W_{\eta \eta}=\frac{-\Gamma_{F}}{4}\left[( 1 + P \operatorname { c o s } \phi ) \left(\delta_{-}^{2}\left[f^{-}\left(E_{\eta}-E_{+-}\right)+f^{+}\left(E_{++}-E_{\eta}\right)\right]+\right.\right. \\
\left.\delta_{+}^{2}\left[f^{-}\left(E_{\eta}-E_{--}\right)+f^{+}\left(E_{-+}-E_{\eta}\right)\right]\right)(1-P \cos \phi)\left(\delta _ { + } ^ { 2 } \left[f^{+}\left(E_{+-}-E_{\eta}\right)\right.\right. \\
\left.\left.\left.+f^{-}\left(E_{\eta}-E_{-+}\right)\right]+\delta_{-}^{2}\left[f^{+}\left(E_{--}-E_{\eta}\right)+f^{-}\left(E_{\eta}-E_{++}\right)\right]\right)\right]
\end{gathered}
$$

and

$$
\begin{aligned}
& W_{ \pm \mp \mp \pm}^{\mp \pm \mp}=\frac{-i \Gamma_{F}}{4 \pi}\left[( 1 + P \operatorname { c o s } \phi ) \left(\delta _ { + } ^ { 2 } \left[-i \pi\left(f^{+}\left(E_{I+}-E_{+-}\right)+f^{-}\left(E_{-+}-E_{I-}\right)\right)\right.\right.\right. \\
& \left. \pm \operatorname{Re}\left[\Psi\left(\frac{1}{2}+\frac{i \beta}{2 \pi}\left(E_{-+}-E_{I-}-\mu_{F}\right)\right)-\Psi\left(\frac{1}{2}+\frac{i \beta}{2 \pi}\left(E_{I+}-E_{+-}-\mu_{F}\right)\right)\right]\right]+ \\
& \frac{\delta_{-}^{2}}{2}\left[-i \pi\left(f^{+}\left(E_{I 0}-E_{-+}\right)+f^{+}\left(E_{I S}-E_{-+}\right)+f^{-}\left(E_{+-}-E_{I 0}\right)+f^{-}\left(E_{+-}-E_{I S}\right)\right)\right. \\
& \pm \operatorname{Re}\left[\Psi\left(\frac{1}{2}+\frac{i \beta}{2 \pi}\left(E_{I 0}-E_{-+}-\mu_{F}\right)\right)-\Psi\left(\frac{1}{2}+\frac{i \beta}{2 \pi}\left(E_{+-}-E_{I 0}-\mu_{F}\right)\right)\right] \\
& \left.\left. \pm \operatorname{Re}\left[\Psi\left(\frac{1}{2}+\frac{i \beta}{2 \pi}\left(E_{I S}-E_{-+}-\mu_{F}\right)\right)-\Psi\left(\frac{1}{2}+\frac{i \beta}{2 \pi}\left(E_{+-}-E_{I S}-\mu_{F}\right)\right)\right]\right]\right) \\
& +(1-P \cos \phi)\left(\delta _ { - } ^ { 2 } \left[-i \pi\left(f^{+}\left(E_{I-}-E_{-+}\right)+f^{-}\left(E_{+-}-E_{I+}\right)\right)\right.\right. \\
& \left. \pm \operatorname{Re}\left[\Psi\left(\frac{1}{2}+\frac{i \beta}{2 \pi}\left(E_{I-}-E_{-+}-\mu_{F}\right)\right)-\Psi\left(\frac{1}{2}+\frac{i \beta}{2 \pi}\left(E_{+-}-E_{I+}-\mu_{F}\right)\right)\right]\right]+ \\
& \frac{\delta_{+}^{2}}{2}\left[-i \pi\left(f^{+}\left(E_{I 0}-E_{+-}\right)+f^{+}\left(E_{I S}-E_{+-}\right)+f^{-}\left(E_{-+}-E_{I 0}\right)+f^{-}\left(E_{-+}-E_{I S}\right)\right)\right. \\
& \pm \operatorname{Re}\left[\Psi\left(\frac{1}{2}+\frac{i \beta}{2 \pi}\left(E_{-+}-E_{I 0}-\mu_{F}\right)\right)-\Psi\left(\frac{1}{2}+\frac{i \beta}{2 \pi}\left(E_{I 0}-E_{+-}-\mu_{F}\right)\right)\right] \\
& \left.\left.\left. \pm \operatorname{Re}\left[\Psi\left(\frac{1}{2}+\frac{i \beta}{2 \pi}\left(E_{-+}-E_{I S}-\mu_{F}\right)\right)-\Psi\left(\frac{1}{2}+\frac{i \beta}{2 \pi}\left(E_{I S}-E_{+-}-\mu_{F}\right)\right)\right]\right]\right)\right],
\end{aligned}
$$

and

$$
W_{ \pm \mp \pm \mp}^{+--+}=W_{ \pm \mp \pm \mp}^{-++-}=\frac{i \delta_{-} \delta_{+}}{8 \pi} \Gamma_{F} P \sin \phi\left[i \pi \left(f^{-}\left(E_{ \pm \mp}-E_{I S}\right)\right.\right.
$$




$$
\begin{gathered}
\left.-f^{+}\left(E_{I S}-E_{ \pm \mp}\right)-f^{-}\left(E_{ \pm \mp}-E_{I 0}\right)+f^{+}\left(E_{I 0}-E_{ \pm \mp}\right)\right) \\
+\operatorname{Re}\left[\Psi\left(\frac{1}{2}+\frac{\beta i}{2 \pi}\left(E_{ \pm \mp}-E_{I 0}-\mu_{F}\right)\right)-\Psi\left(\frac{1}{2}+\frac{\beta i}{2 \pi}\left(E_{ \pm \mp}-E_{I S}-\mu_{F}\right)\right)\right] \\
+\operatorname{Re}\left[\Psi\left(\frac{1}{2}+\frac{\beta i}{2 \pi}\left(E_{I 0}-E_{ \pm \mp}-\mu_{F}\right)\right)-\Psi\left(\frac{1}{2}+\frac{\beta i}{2 \pi}\left(E_{I S}-E_{ \pm \mp}-\mu_{F}\right)\right)\right], \\
W_{+--+}^{ \pm \mp \pm \mp}=W_{-+\mp \mp}^{ \pm \mp}=\frac{i \delta_{-} \delta_{+}}{8 \pi} \Gamma_{F} P \sin \phi\left[i \pi \left(f^{-}\left(E_{ \pm \mp}-E_{I 0}\right)\right.\right. \\
\left.-f^{+}\left(E_{I 0}-E_{ \pm \mp}\right)-f^{-}\left(E_{ \pm \mp}-E_{I S}\right)+f^{+}\left(E_{I S}-E_{ \pm \mp}\right)\right) \\
-\operatorname{Re}\left[\Psi\left(\frac{1}{2}+\frac{\beta i}{2 \pi}\left(E_{ \pm \mp}-E_{I 0}-\mu_{F}\right)\right)-\Psi\left(\frac{1}{2}+\frac{\beta i}{2 \pi}\left(E_{ \pm \mp}-E_{I S}-\mu_{F}\right)\right)\right] \\
-\operatorname{Re}\left[\Psi\left(\frac{1}{2}+\frac{\beta i}{2 \pi}\left(E_{I 0}-E_{ \pm \mp}-\mu_{F}\right)\right)-\Psi\left(\frac{1}{2}+\frac{\beta i}{2 \pi}\left(E_{I S}-E_{ \pm \mp}-\mu_{F}\right)\right)\right] .
\end{gathered}
$$




\section{Bibliography}

[1] P. Shor, "Algorithms for quantum computation: discrete logarithms and factoring," in Foundations of Computer Science, 1994 Proceedings., 35th Annual Symposium on, pp. 124-134, IEEE, 1994.

[2] J. J. L. Morton, A. M. Tyryshkin, A. Ardavan, S. C. Benjamin, K. Porfyrakis, S. A. Lyon, and G. A. D. Briggs, "The $\mathrm{n}_{\mathrm{c}} \mathrm{c}_{60}$ nuclear spin qubit: Bang-bang decoupling and ultrafast phase gates," Phys. Status Solidi B, vol. 243, pp. 3028-3031, Nov 2006.

[3] S. De Franceschi, L. Kouwenhoven, C. Schonenberger, and W. Wernsdorfer, "Hybrid superconductor-quantum dot devices," Nat Nano, vol. 5, pp. 703-711, 2010.

[4] D. Bimberg, "Quantum dot based nanophotonics and nanoelectronics," Electronics Letters, vol. 44, pp. 168 -171, 312008.

[5] M. Governale, M. G. Pala, and J. König, "Real-time diagrammatic approach to transport through interacting quantum dots with normal and superconducting leads," Phys. Rev. B, vol. 77, p. 134513, Apr 2008.

[6] A. Braggio, J. König, and R. Fazio, "Full counting statistics in strongly interacting systems: Non-markovian effects," Phys. Rev. Lett., vol. 96, p. 026805, Jan 2006.

[7] S. Gustavsson, R. Leturcq, B. Simovič, R. Schleser, T. Ihn, P. Studerus, K. Ensslin, D. C. Driscoll, and A. C. Gossard, "Counting statistics of single electron transport in a quantum dot," Phys. Rev. Lett., vol. 96, p. 076605, Feb 2006.

[8] J. J. Parks, A. R. Champagne, T. A. Costi, W. W. Shum, A. N. Pasupathy, E. Neuscamman, S. Flores-Torres, P. S. Cornaglia, A. A. Aligia, C. A. Balseiro, G. K.-L. Chan, H. D. Abruna, and D. C. Ralph, "Mechanical control of spin states in spin-1 molecules and the underscreened kondo effect," Science, vol. 328, no. 5984 , pp. $1370-1373,2010$. 
[9] A. Zyazin, J. van den Berg, E. Osorio, H. van der Zant, N. Konstantinidis, M. Leijnse, M. Wegewijs, F. May, W. Hofstetter, C. Danieli, and A. Cornia, "Electric field controlled magnetic anisotropy in a single molecule," Nano letters, vol. 10, pp. 3307-3311, Sep 2010.

[10] D. Gatteschi and R. Sessoli, "Quantum tunneling of magnetization and related phenomena in molecular materials," Angewandte Chemie, vol. 42, pp. 268-297, 2003.

[11] N. Roch, R. Vincent, F. Elste, W. Harneit, W. Wernsdorfer, C. Timm, and F. Balestro, "Cotunneling through a magnetic single-molecule transistor based on n@c ${ }_{60}$," Phys. Rev. B, vol. 83, p. 081407, Feb 2011.

[12] H. B. Heersche, Z. de Groot, J. A. Folk, H. S. J. van der Zant, C. Romeike, M. R. Wegewijs, L. Zobbi, D. Barreca, E. Tondello, and A. Cornia, "Electron transport through single Mn_12 molecular magnets," Phys. Rev. Lett., vol. 96, p. 206801, May 2006.

[13] C. B. Winkelmann, N. Roch, W. Wernsdorfer, V. Bouchiat, and F. Balestro, "Superconductivity in single-c 60 transistor," Nature Physics, vol. 5, pp. 876-879, 2009.

[14] B. D. Josephson, "Possible new effects in superconductive tunnelling," Physics Letters, vol. 1, pp. 251-253, Jul 1962.

[15] J. Kondo, "Resistance minimum in dilute magnetic alloys," Progress of Theoretical Physics, vol. 32, no. 1, pp. 37-49, 1964.

[16] L. Glazman and M. Pustilnik, "Coulomb blockade and kondo effect in quantum dots," in New Directions in Mesoscopic Physics (Towards Nanoscience) (R. Fazio, V. Gantmakher, and Y. Imry, eds.), vol. 125 of NATO Science Series, pp. 93-115, Springer Netherlands, 2003.

[17] R. Fazio and R. Raimondi, "Resonant andreev tunneling in strongly interacting quantum dots," Phys. Rev. Lett., vol. 80, pp. 2913-2916, Mar 1998.

[18] K. Kang, "Kondo resonance, coulomb blockade, and andreev transport through a quantum dot," Phys. Rev. B, vol. 58, pp. 9641-9643, Oct 1998.

[19] I. Kulik, "Macroscopic quantization and proximity effect in s-n-s junctions," Sov. Phys. JETP-USSR, vol. 30, pp. 944-947, 1970. 
[20] M. Lee, T. Jonckheere, and T. Martin, "Josephson effect through an isotropic magnetic molecule," Phys. Rev. Lett., vol. 101, p. 146804, Oct 2008.

[21] I. A. Sadovskyy, D. Chevallier, T. Jonckheere, M. Lee, S. Kawabata, and T. Martin, "Josephson effect through an anisotropic magnetic molecule," Phys. Rev. B, vol. 84, p. 184513, Nov 2011.

[22] F. Elste and C. Timm, "Transport through anisotropic magnetic molecules with partially ferromagnetic leads: Spin-charge conversion and negative differential conductance," Phys. Rev. B, vol. 73, p. 235305, Jun 2006.

[23] C. Romeike, M. R. Wegewijs, W. Hofstetter, and H. Schoeller, "Quantumtunneling-induced kondo effect in single molecular magnets," Phys. Rev. Lett., vol. 96, p. 196601, May 2006.

[24] B. Barbara and L. Gunther, "Magnets, molecules and quantum mechanics," Phys. World, pp. 35-40, Mar 1999.

[25] R. Sessoli, D. Gatteschi, A. Caneschi, and M. A. Novak, "Magnetic bistability in a metal-ion cluster," Nature, vol. 365, pp. 141-143, Sep 1993.

[26] C. Sangregorio, T. Ohm, C. Paulsen, R. Sessoli, and D. Gatteschi, "Quantum tunneling of the magnetization in an iron cluster nanomagnet," Phys. Rev. Lett., vol. 78, pp. 4645-4648, Jun 1997.

[27] M.-H. Jo, J. E. Grose, K. Baheti, M. M. Deshmukh, J. J. Sokol, E. M. Rumberger, D. N. Hendrickson, J. R. Long, H. Park, and D. C. Ralph, "Signatures of molecular magnetism in single-molecule transport spectroscopy," Nano Letters, vol. 6, no. 9, pp. 2014-2020, 2006.

[28] P. W. Anderson, "Localized magnetic states in metals," Phys. Rev., vol. 124, pp. 41-53, Oct 1961.

[29] L. P. Kouwenhoven, D. G. Austing, and S. Tarucha, "Few-electron quantum dots," Reports on Progress in Physics, vol. 64, no. 6, p. 701, 2001.

[30] R. C. Ashoori, "Electrons in artificial atoms," Nature, vol. 379, p. 413, Feb 1996.

[31] D. Bimberg, E. Stock, A. Lochmann, A. Schliwa, W. Unrau, M. Münnix, S. Rodt, A. I. Toropov, A. Bakarov, A. K. Kalagin, and V. A. Haisler, "Quantum dots for single and entangled photon emitters," pp. 76100G-76100G-13, 2010. 
[32] T. D. Ladd, F. Jelezko, R. Laflamme, Y. Nakamura, C. Monroe, and J. L. O'Brien, "Quantum computers," Nature, vol. 464, p. 45, Mar 2010.

[33] M. Kroutvar, Y. Ducommun, D. Heiss, M. Bichler, D. Schuh, G. Abstreiter, and J. J. Finley, "Optically programmable electron spin memory using semiconductor quantum dots," Nature, vol. 432, p. 81, Nov 2004.

[34] D. Loss and D. P. DiVincenzo, "Quantum computation with quantum dots," Phys. Rev. A, vol. 57, pp. 120-126, Jan 1998.

[35] R. Hanson, L. P. Kouwenhoven, J. R. Petta, S. Tarucha, and L. M. K. Vandersypen, "Spins in few-electron quantum dots," Rev. Mod. Phys., vol. 79, pp. 12171265 , Oct 2007.

[36] H. Onnes Leiden Comm 120b, 122b, 124c, 1911.

[37] W. Meissner and R. Ochsenfeld, "Ein neuer effekt bei eintritt der supraleitfähigkeit," Naturwissenschaften, vol. 21, no. 44, pp. 787-788, 1933.

[38] J. Bardeen, L. N. Cooper, and J. R. Schrieffer, "Theory of superconductivity," Phys. Rev., vol. 108, pp. 1175-1204, Dec 1957.

[39] M. Tinkham, Introduction to Superconductivity. Dover books on physics and chemistry, Dover Publications, 2004.

[40] L. N. Cooper, "Bound electron pairs in a degenerate fermi gas," Phys. Rev., vol. 104, pp. 1189-1190, Nov 1956.

[41] W. L. McMillan, "Tunneling model of the superconducting proximity effect," Phys. Rev., vol. 175, pp. 537-542, Nov 1968.

[42] R. Holm and W. Meissner, "Messungen mit hilfe von flüssigem helium. xiii," Zeitschrift für Physik, vol. 74, pp. 715-735, 1932.

[43] P. de Gennes and D. Saint-James, "Elementary excitations in the vicinity of a normal metal-superconducting metal contact," Physics Letters, vol. 4, no. 2, pp. 151 $-152,1963$.

[44] A. Andreev JETP, vol. 19, p. 1228, 1964. 
[45] L. Hofstetter, A. Geresdi, M. Aagesen, J. Nygård, C. Schönenberger, and S. Csonka, "Ferromagnetic proximity effect in a ferromagnet quantum-dot superconductor device," Phys. Rev. Lett., vol. 104, p. 246804, Jun 2010.

[46] B. Sothmann, D. Futterer, M. Governale, and J. König, "Probing the exchange field of a quantum-dot spin valve by a superconducting lead," Phys. Rev. B, vol. 82, p. 094514, Sep 2010.

[47] A. Braggio, M. Governale, M. G. Pala, and J. König, "Superconducting proximity effect in interacting quantum dots revealed by shot noise," Solid State Communications, vol. 151, no. 2, pp. 155 - 158, 2011.

[48] C. Romeike, M. R. Wegewijs, and H. Schoeller, "Spin quantum tunneling in single molecular magnets: Fingerprints in transport spectroscopy of current and noise," Phys. Rev. Lett., vol. 96, p. 196805, May 2006.

[49] J. König, Quantenfluktuationen im Einzel-Elektronen-Transistor. PhD thesis, Universität Karlsruhe, December 1998.

[50] A. V. Rozhkov and D. P. Arovas, "Interacting-impurity josephson junction: Variational wave functions and slave-boson mean-field theory," Phys. Rev. B, vol. 62, pp. 6687-6691, Sep 2000. 